\title{
A REFINEMENT OF SUTURED FLOER HOMOLOGY
}

\author{
AKRAM S. ALISHAHI AND EAMAN EFTEKHARY
}

\begin{abstract}
We introduce a refinement of the Ozsváth-Szabó complex associated to a balanced sutured manifold $(X, \tau)$ by Juhász [Ju1]. An algebra $\mathbb{A}_{\tau}$ is associated to the boundary of a sutured manifold and a filtration of its generators by $\mathrm{H}^{2}(X, \partial X ; \mathbb{Z})$ is defined. For a fixed class $\mathfrak{s}$ of a Spin ${ }^{c}$ structure over the manifold $\bar{X}$, which is obtained from $X$ by filling out the sutures, the Ozsváth-Szabó chain complex $\mathrm{CF}(X, \tau, \mathfrak{s})$ is then defined as a chain complex with coefficients in $\mathbb{A}_{\tau}$ and filtered by $\operatorname{Spin}^{c}(X, \tau)$. The filtered chain homotopy type of this chain complex is an invariant of $(X, \tau)$ and the $\operatorname{Spin}^{c}$ class $\mathfrak{s} \in \operatorname{Spin}^{c}(\bar{X})$. The construction generalizes the construction of Juhász. It plays the role of $\mathrm{CF}^{-}(X, \mathfrak{s})$ when $X$ is a closed three-manifold, and the role of

$$
\operatorname{CFK}^{-}(Y, K ; \mathfrak{s})=\bigoplus_{\underline{\mathfrak{s}} \in \mathfrak{s}} \mathrm{CFK}^{-}(Y, K, \underline{\mathfrak{s}}),
$$

when the sutured manifold is obtained from a knot $K$ inside a three-manifold $Y$. Our invariants generalize both the knot invariants of Ozsváth-Szabó and Rasmussen and the link invariants of Ozsváth and Szabó. We study some of the basic properties of the corresponding Ozsváth-Szabó complex, including the exact triangles, and some form of stabilization.
\end{abstract}

\section{Contents}

1. Introduction

1.1. Introduction and the main results

1.2. Previous results and the history

1.3. Outline of the paper

2. Background on sutured manifolds

2.1. Sutured manifolds and relative $\operatorname{Spin}^{c}$ structures

2.2. Associated sutured manifolds; surgery and filling the sutures

2.3. Relative Spin ${ }^{c}$-structures and Heegaard diagrams

3. Algebra input

3.1. The $\mathbb{A}$-chain complexes

3.2. The mapping cones of $\mathbb{A}$-chain maps

3.3. Filtration by a $\mathbb{Z}$-module

3.4. The algebra associated with the boundary of a sutured manifold

4. Admissible Heegaard diagrams

4.1. The notion of $\mathfrak{s}$-admissibility

4.2. Existence of $\mathfrak{s}$-admissible Heegaard diagrams

5. The chain complex associated with a balanced sutured manifold

5.1. Holomorphic disks and boundary degenerations; orientation issues

5.2. Energy bounds and relative gradings

5.3. The construction of the chain complex

5.4. Additional algebraic structure 
6. Invariance of the filtered chain homotopy type 46

6.1. Pseudo-holomorphic $m$-gons 46

6.2. Special Heegaard diagrams corresponding to handle slides 53

6.3. The triangle map and the invariance 57

7. Stabilization of sutured manifolds 65

7.1. Simple stabilization of a balanced sutured manifold 65

7.2. The analytic input 67

7.3. Proof of the stabilization formula 70

8. A triangle associated with surgery 75

8.1. The triangle associated with the surgery Heegaard quadruple 75

8.2. Compositions in the triangle are null-homotopic 84

8.3. Exactness and computation of chain homotopy type 87

8.4. Special cases 90

References 


\section{INTRODUCTION}

1.1. Introduction and the main results. The introduction of Heegaard Floer homology by Ozsváth and Szabó ( OS5, OS5]) for closed three dimensional manifolds around the beginning of the millennium resulted in very powerful tools for the study of various structures in low dimensional topology. In particular, invariants for knots (c.f. OS1, Ras1 and Ef2), for links OS8, and for contact structures OS10 were constructed using the fundamental idea of associating a chain complex to a pointed Heegaard diagram. Moreover, four manifold invariants were constructed as some TQFT type homomorphisms between the homology groups of the chain complexes associated to the positive and negative boundary components OS11. The Ozsváth-Szabó complexes associated with a closed three-manifold come in different flavors. These are typically called hat, minus, plus and infinity modules. The other versions may be re-constructed from the minus theory if one also keeps track of the so called $U$-action. Juhász extended the hat version of Ozsváth-Szabó complex to the context of balanced sutured manifolds [Ju1]. The sutured Floer homology of Juhász detects taut sutured manifolds [Ju2, and may be used to define a polytope associated with a sutured manifold which behaves well under taut surface decompositions [Ju3].

In this paper, we extend the construction of Juhász and construct a minus theory associated with a balanced sutured manifold. More precisely, let $(X, \tau)$ be a balanced sutured manifold and let $\tau=\left\{\gamma_{1}, \ldots, \gamma_{\kappa}\right\}$ be the set of sutures. We will denote $\partial X-\tau$ by $\mathfrak{R}(\tau)=\mathfrak{R}^{+}(\tau) \cup \mathfrak{R}^{-}(\tau)$, where $\mathfrak{R}^{+}(\tau)$ and $\mathfrak{R}^{-}(\tau)$ are the positive and the negative part of the boundary, respectively. We first associate an algebra $\mathbb{A}=\mathbb{A}_{\tau}$ to the boundary of $X$ as follows. Let us assume that

$$
\mathfrak{R}^{-}(\tau)=\bigcup_{i=1}^{k} R_{i}^{-}, \quad \& \quad \mathfrak{R}^{+}(\tau)=\bigcup_{j=1}^{l} R_{j}^{+},
$$

where $R_{i}^{-}$and $R_{j}^{+}$are the connected components of $\mathfrak{R}^{-}(\tau)$ and $\mathfrak{R}^{+}(\tau)$ respectively. Let $g_{i}^{-}$denote the genus of $R_{i}^{-}$and $g_{j}^{+}$denote the genus of $R_{j}^{+}$. Consider the elements

$$
\lambda_{i}^{-}:=\prod_{\gamma_{j} \subset \partial R_{i}^{-}} \lambda_{j}, \quad i=1, \ldots, k, \quad \& \quad \lambda_{i}^{+}:=\prod_{\gamma_{j} \subset \partial R_{i}^{+}} \lambda_{j}, \quad i=1, \ldots, l,
$$

in the free $\mathbb{Z}$-algebra $\mathbb{Z}[\kappa]:=\left\langle\lambda_{1}, \ldots, \lambda_{\kappa}\right\rangle$ generated by $\lambda_{1}, \ldots, \lambda_{\kappa}$. Let

$$
\begin{aligned}
& \mathbb{A}_{\tau}:=\frac{\left\langle\lambda_{1}, \ldots, \lambda_{\kappa}\right\rangle_{\mathbb{Z}}}{\left\langle\lambda^{+}(\tau)-\lambda^{-}(\tau)\right\rangle+\left\langle\lambda_{i}^{+} \mid g_{i}^{+}>0\right\rangle+\left\langle\lambda_{j}^{-} \mid g_{j}^{-}>0\right\rangle}, \\
& \text { where } \lambda^{-}(\tau)=\sum_{i=1}^{k} \lambda_{i}^{-}, \quad \& \quad \lambda^{+}(\tau)=\sum_{i=1}^{l} \lambda_{i}^{+} .
\end{aligned}
$$

We will denote the set of monomials $\prod_{i=1}^{\kappa} \lambda_{i}^{a_{i}}$ by $G(\mathbb{A})$, which forms a set of generators for $\mathbb{A}$. One may define a natural morphism from $G(\mathbb{A})$ to the $\mathbb{Z}$-module 


$$
\begin{aligned}
\mathbb{H}=\mathbb{H}_{\tau}:= & \mathrm{H}^{2}(X, \partial X, \mathbb{Z}) \text { by } \\
& \chi: G(\mathbb{A}) \longrightarrow \mathbb{H}=\mathrm{H}^{2}(X, \partial X ; \mathbb{Z}), \\
& \chi\left(\prod_{i=0}^{\kappa} \lambda_{i}^{a_{i}}\right):=a_{1} \operatorname{PD}\left[\gamma_{1}\right]+\ldots+a_{\kappa} \operatorname{PD}\left[\gamma_{\kappa}\right], \quad \forall a_{1}, . ., a_{\kappa} \in \mathbb{Z}^{\geq 0} .
\end{aligned}
$$

Let $\bar{X}=\bar{X}^{\tau}$ be the three-manifold obtained by filling the sutures of $(X, \tau)$ by attaching 2-handles to the sutures in $\tau$. Fix a $\operatorname{Spin}^{c}$ class $\mathfrak{s} \in \operatorname{Spin}^{c}(\bar{X})$. Suppose that $(\Sigma, \boldsymbol{\alpha}, \boldsymbol{\beta}, \mathbf{z})$ is a Heegaard diagram for the sutured manifold $(X, \tau)$, which is admissible in an appropriate sense. Thus $\Sigma$ is a closed Riemann surface, $\boldsymbol{\alpha}$ and $\boldsymbol{\beta}$ are $\ell$-tuples of disjoint simple closed curves, and $\mathbf{z}$ is a set of $\kappa$ marked points on $\Sigma$. If $\Sigma^{\circ}=\Sigma-\operatorname{nd}(\mathbf{z})$ is the complement of a neighborhood of $\mathbf{z}, X$ is obtained from $\Sigma^{\circ} \times[-1,1]$ by attaching 2-handles to $\boldsymbol{\alpha} \times\{-1\}$ and $\boldsymbol{\beta} \times\{1\}$. The OzsváthSzabó chain complex $\operatorname{CF}(X, \tau, \mathfrak{s})$ is then generated as a free $\mathbb{A}_{\tau}$-module by those intersection points of the tori $\mathbb{T}_{\alpha}, \mathbb{T}_{\beta} \subset \operatorname{Sym}^{\ell}(\Sigma)$ associated with $\boldsymbol{\alpha}$ and $\boldsymbol{\beta}$ which correspond to the $\operatorname{Spin}^{c}$ class $\mathfrak{s} \in \operatorname{Spin}^{c}(\bar{X})$. The set $\pi_{2}^{+}(\mathbf{x}, \mathbf{y})$ of positive Whitney disks for generators $\mathbf{x}, \mathbf{y} \in \mathbb{T}_{\alpha} \cap \mathbb{T}_{\beta}$ is defined as usual, and we will have a map

$$
\begin{aligned}
& \lambda_{\mathbf{z}}: \coprod_{\mathbf{x}, \mathbf{y} \in \mathbb{T}_{\alpha} \cap \mathbb{T}_{\beta}} \pi_{2}^{+}(\mathbf{x}, \mathbf{y}) \longrightarrow G(\mathbb{A}) \\
& \lambda_{\mathbf{z}}(\phi):=\prod_{i=1}^{\kappa} \lambda_{i}^{n_{z_{i}}(\phi)}, \quad \forall \mathbf{x}, \mathbf{y} \in \mathbb{T}_{\alpha} \cap \mathbb{T}_{\beta}, \& \forall \phi \in \pi_{2}^{+}(\mathbf{x}, \mathbf{y}) .
\end{aligned}
$$

Here $n_{z_{i}}(\phi)$ denotes the coefficient of $z_{i}$ in the domain $\mathcal{D}(\phi)$ associated with the Whitney disk $\phi$. The differential $\partial$ of the complex $\mathrm{CF}(X, \tau, \mathfrak{s})$ is defined by counting holomorphic disks $\phi$ of Maslov index 1 connecting the generators $\mathbf{x}$ and $\mathbf{y}$ of the complex, with an appropriate sign and weight $\lambda_{\mathbf{z}}(\phi) \in \mathbb{A}_{\tau}$. The assignment of relative $\operatorname{Spin}^{c}$ structures to the intersection points $\mathbf{x} \in \mathbb{T}_{\alpha} \cap \mathbb{T}_{\beta}$ using z gives $\mathrm{CF}(X, \tau, \mathfrak{s})$ the structure of a filtered $(\mathbb{A}, \mathbb{H})$ chain complex (see section 3 for a precise definition). The following is the main result of this paper.

Theorem 1.1. The filtered $(\mathbb{A}, \mathbb{H})$ chain homotopy type of the filtered $(\mathbb{A}, \mathbb{H})$ chain complex $\mathrm{CF}(X, \tau, \mathfrak{s})$ is an invariant of the balanced sutured manifold $(X, \tau)$ and the $\operatorname{Spin}^{c}$ class $\mathfrak{s} \in \operatorname{Spin}^{c}(\bar{X})$. In particular, for any $\mathfrak{s} \in \mathfrak{s} \subset \operatorname{Spin}^{c}(X, \tau)$ the chain homotopy type of the summand

$$
\mathrm{CF}(X, \tau, \mathfrak{\mathfrak { s }}) \subset \mathrm{CF}(X, \tau, \mathfrak{s})=\bigoplus_{\mathfrak{s} \in \mathfrak{s}} \mathrm{CF}(X, \tau, \underline{\mathfrak{s}})
$$

is also an invariant of $(X, \tau, \mathfrak{s})$.

The above theorem implies that whenever we have a homomorphism $\rho: \mathbb{A} \rightarrow \mathbb{B}$ for a ring $\mathbb{B}$, the chain homotopy type of the complex

$$
\mathrm{CF}(X, \tau, \mathfrak{s} ; \mathbb{B})=\mathrm{CF}(X, \tau, \mathfrak{s}) \otimes_{\mathbb{A}} \mathbb{B}
$$

is also an invariant of the sutured manifold $(X, \tau)$. This complex is equipped with filtration by $\operatorname{Spin}^{c}(X, \tau)$ if the homomorphism $\rho$ respects the filtration of the monomials of $\mathbb{A}$ by the elements of $\mathbb{H}$. In this case, it makes sense to talk about the following decomposition of $\mathrm{CF}(X, \tau, \mathfrak{s} ; \mathbb{B})$ :

$$
\mathrm{CF}(X, \tau, \mathfrak{s} ; \mathbb{B})=\bigoplus_{\underline{\mathfrak{s}} \in \mathfrak{s} \subset \operatorname{Spin}^{c}(X, \tau)} \mathrm{CF}(X, \tau, \mathfrak{s} ; \mathbb{B}) .
$$


In particular, the homology groups

$$
\operatorname{HF}(X, \tau, \underline{\mathfrak{s}} ; \mathbb{B})=H_{*}(\mathrm{CF}(X, \tau, \underline{\mathfrak{s}} ; \mathbb{B}), \partial), \quad \forall \underline{\mathfrak{s}} \in \operatorname{Spin}^{c}(X, \tau)
$$

may be defined, and are invariants of the sutured manifold and the relative $\operatorname{Spin}^{c}$ class $\mathfrak{s} \in \operatorname{Spin}^{c}(X, \tau)$. As a special case, we may take $\mathbb{B}=\mathbb{Z}$ and let $\rho$ be the map sending all the non-trivial monomials to zero. We will then recover the sutured Floer homology of Juhász:

$$
\operatorname{SFH}(X, \tau, \underline{\mathfrak{s}})=\operatorname{HF}(X, \tau, \underline{\mathfrak{s}} ; \mathbb{Z}), \quad \forall \underline{\mathfrak{s}} \in \operatorname{Spin}^{c}(X, \tau) .
$$

Define a particular test ring $\mathbb{B}_{\tau}$ for $\mathbb{A}_{\tau}$ by setting

$$
\mathbb{B}_{\tau}=\frac{\left\langle\lambda_{1}, \ldots, \lambda_{\kappa}\right\rangle_{\mathbb{Z}}}{\left.\left\langle\prod_{i=1}^{\kappa} \lambda_{i}^{n_{i}} \neq 1\right| n_{i} \in \mathbb{Z} \geq 0 \& \sum_{i=1}^{\kappa} n_{i}\left[\gamma_{i}\right]=0 \text { in } \mathrm{H}_{1}(X ; \mathbb{Z}) / \text { Tors }\right\rangle} .
$$

Clearly, there is a quotient map $\rho_{\tau}: \mathbb{A}_{\tau} \rightarrow \mathbb{B}_{\tau}$. The following is a refinement of Juhász' theorem 1.4 from [Ju2].

Proposition 1.2. An irreducible balanced sutured manifold $(X, \tau)$ is taut if and only if the filtered $\left(\mathbb{B}_{\tau}, \mathbb{H}_{\tau}\right)$ chain homotopy type of the complex

$$
\mathrm{CF}\left(X, \tau ; \mathbb{B}_{\tau}\right)=\bigoplus_{\mathfrak{s} \in \operatorname{Spin}^{c}(\bar{X})} \mathrm{CF}\left(X, \tau ; \mathfrak{s} ; \mathbb{B}_{\tau}\right)
$$

is non-trivial.

For a knot $K$ inside a closed three-manifold $Y$, the boundary of the corresponding sutured manifold $(X=Y-\operatorname{nd}(K), \tau)$ consists of a torus and $\tau$ consists of a pair of parallel sutures on this torus. Thus, with the above notation,

$$
k=l=1, \quad g_{1}^{+}=g_{1}^{-}=0, \quad \& \quad \lambda_{1}^{+}=\lambda_{1}^{-}=\lambda_{1} \lambda_{2} .
$$

Thus, the algebra $\mathbb{A}$ is equal to $\mathbb{Z}\left[\lambda_{1}, \lambda_{2}\right]=\left\langle\lambda_{1}, \lambda_{2}\right\rangle_{\mathbb{Z}}$, which gives the $\mathbb{Z} \oplus \mathbb{Z}$ filtration associated with the knot $K$ inside the three-manifold $Y$.

The surgery exact triangle for the Ozsváth-Szabó complexes associated with closed three-manifolds may be extended to our setup. Namely, let $(X, \tau)$ be a sutured manifold and $\gamma_{1}, \gamma_{2} \in \tau$ be two parallel sutures with opposite orientation which form the common boundary of a cylindrical component $R_{1}^{+} \subset \mathfrak{R}^{+}(\tau)$ and a genus zero component $R_{1}^{-} \subset \mathfrak{R}^{-}(\tau)$. Consider a simple closed curve

$$
\lambda \subset R_{1}^{+} \cup R_{1}^{-} \cup \gamma_{1} \cup \gamma_{2}
$$

which cuts $\gamma_{1}$ and $\gamma_{2}$ in a single transverse point and remains disjoint from the rest of the sutures. Replacing $\gamma_{1}$ and $\gamma_{2}$ with two parallel copies of $\lambda$ (with opposite orientation) results in a new sutured manifold $\left(X, \tau_{\lambda}\right)$. Let $\lambda(n)$ be (the homotopy class of) the simple closed curve obtained from $\lambda$ by twisting it $n$ times along $\gamma_{1}$ (or equivalently, $-n$ times along $\gamma_{2}$ ). Correspondingly, we obtain the sutured manifold $\left(X, \tau_{\lambda(n)}\right)$. When the choice of $\lambda$ is fixed, we sometimes write $\left(X, \tau_{n}\right)$ for $\left(X, \tau_{\lambda(n)}\right)$.

The algebra associated with all the sutured manifolds $\left(X, \tau_{n}\right)$ is the same. Let us denote this algebra by $\mathbb{A}$, and assume that $\zeta_{1}, \ldots, \zeta_{\kappa}$ are the generators of $\mathbb{A}$ which correspond to the sutures. Furthermore, let $\zeta_{1}$ and $\zeta_{2}$ correspond to $\gamma_{1}$ and $\gamma_{2}$ respectively. Note that in the relations ideal $I_{\tau}$ in $\mathbb{Z}[\kappa]=\left\langle\zeta_{1}, \ldots, \zeta_{\kappa}\right\rangle_{\mathbb{Z}}$ (which defines $\mathbb{A}$ as $\left.\mathbb{Z}[\kappa] / I_{\tau}\right)$ the generators either use $\zeta_{1} \zeta_{2}$, or they use none of $\zeta_{1}$ and $\zeta_{2}$. 
We may thus introduce a new algebra $\mathbb{B}$ as a quotient of $\left\langle\lambda_{0}, \lambda_{1}, \ldots, \lambda_{\kappa}\right\rangle_{\mathbb{Z}}$ by an ideal $J_{\tau}$. The generators of $J_{\tau}$ are constructed from the generators of $I_{\tau}$ by replacing $\zeta_{j}$ with $\lambda_{j}$ for $j=3, \ldots, \kappa$ and replacing $\zeta_{1} \zeta_{2}$ with $\lambda_{0} \lambda_{1} \lambda_{2}$. For $i=0,1,2$ we obtain embeddings $\imath^{i}$ of $\mathbb{A}$ in $\mathbb{B}$ :

$$
\imath^{i}: \mathbb{A} \rightarrow \mathbb{B}, \quad i^{i}\left(\zeta_{j}\right)=\left\{\begin{array}{ll}
\lambda_{i} & \text { if } j=1 \\
\frac{\lambda_{0} \lambda_{1} \lambda_{2}}{\lambda_{i}} & \text { if } j=2 \\
\lambda_{j} & \text { if } 3 \leq j \leq \kappa
\end{array} .\right.
$$

We write $\mathbb{A}_{i}$ in order to refer to $\mathbb{A}$ as the sub-ring $\imath^{i}(\mathbb{A}) \subset \mathbb{B}$.

To keep the exposition simpler, we only consider the surgery triangle associated with the sutured manifolds $(X, \tau),\left(X, \tau_{0}\right)$ and $\left(X, \tau_{1}\right)$. Let us denote by $\chi_{j} \in$ $\mathrm{H}^{2}(X, \partial X ; \mathbb{Z})$ the Poincaré dual of the suture $\gamma_{j}$, for $j=3, \ldots, \kappa$. Furthermore, let $\chi_{0}, \chi_{1}$ and $\chi_{2}$ denote the Poincaré duals of $\gamma_{1}, \lambda(0)$ and $-\lambda(1)$, respectively. Note that $\chi_{0}+\chi_{1}+\chi_{2}=0$ in $\mathbb{H}=\mathrm{H}^{2}(X, \partial X ; \mathbb{Z})$. Define the filtration map by

$$
\begin{aligned}
& \chi: G(\mathbb{B}) \longrightarrow \mathrm{H}^{2}(X, \partial X ; \mathbb{Z}) \\
& \chi\left(\prod_{j=0}^{\kappa} \lambda_{i}^{a_{i}}\right):=\sum_{j=0}^{\kappa} a_{i} \chi_{i} .
\end{aligned}
$$

Associated with any $\operatorname{Spin}^{c}$ class $\mathfrak{s} \in \operatorname{Spin}^{c}(\bar{X})$ let $\mathbb{E}_{i}\left(\mathfrak{s} ; \mathbb{A}_{i}\right)$ be the complex $\operatorname{CF}\left(X, \tau, \mathfrak{s} ; \mathbb{A}_{0}\right)$, $\mathrm{CF}\left(X, \tau_{0}, \mathfrak{s} ; \mathbb{A}_{1}\right)$, or $\mathrm{CF}\left(X, \tau_{1}, \mathfrak{s} ; \mathbb{A}_{2}\right)$ depending on whether $i=0,1$ or 2 . Let $\mathbb{E}_{i}(\mathfrak{s} ; \mathbb{B})=\mathbb{E}_{i}\left(\mathfrak{s} ; \mathbb{A}_{i}\right) \otimes_{\mathbb{A}_{i}} \mathbb{B}$.

Theorem 1.3. With the above notation fixed, we have a triangle

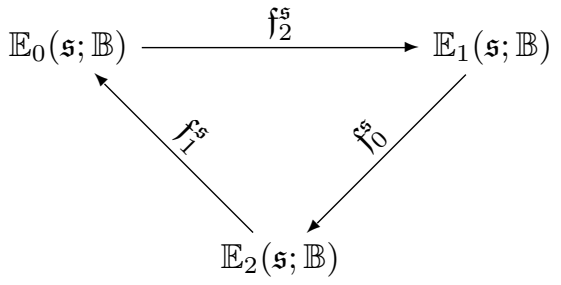

of filtered $(\mathbb{B}, \mathbb{H})$ chain maps such that $\mathfrak{f}_{1}^{\mathfrak{s}} \circ \mathfrak{f}_{0}^{\mathfrak{s}}, \mathfrak{f}_{2}^{\mathfrak{s}} \circ \mathfrak{f}_{1}^{\mathfrak{s}}$, and $\mathfrak{f}_{0}^{\mathfrak{s}} \circ \mathfrak{f}_{2}^{\mathfrak{s}}$ are null homotopic. Moreover, $\mathbb{E}_{i}(\mathfrak{s} ; \mathbb{B})$ is filtered $(\mathbb{B}, \mathbb{H})$ chain homotopic to the mapping cone of $\mathfrak{f}_{i}^{\mathfrak{s}}$. In particular, if there is a homomorphism $\rho_{R}: \mathbb{B} \rightarrow R$ to a ring $R$, taking the tensor product of the above triangle with $R$ and computing the homology groups we obtain a long exact sequence in homology:

$$
\ldots \stackrel{f_{1}^{\mathfrak{s}}}{\longrightarrow} \mathrm{HF}(X, \tau, \mathfrak{s} ; R) \stackrel{\mathfrak{f}_{2}^{\mathfrak{s}}}{\longrightarrow} \mathrm{HF}\left(X, \tau_{0}, \mathfrak{s} ; R\right) \stackrel{\mathfrak{f}_{0}^{\mathfrak{s}}}{\longrightarrow} \mathrm{HF}\left(X, \tau_{1}, \mathfrak{s} ; R\right) \stackrel{\mathfrak{f}_{1}^{\mathfrak{s}}}{\longrightarrow} \ldots
$$

If the homomorphism $\rho_{R}$ also respects the filtration by $\mathbb{H}$, the above exact sequence refines to an exact sequence corresponding to any of the relative $\operatorname{Spin}^{c}$ structures $\underline{\mathfrak{s}} \in \operatorname{Spin}^{c}(X, \tau)$.

1.2. Previous results and the history. Attempts on extending the OzsváthSzabó invariants to three manifolds with boundary, at least when the boundary is equipped with some extra structure have been made through two different approaches. If a parametrization of the boundary surface is fixed, the three-manifold 
is called a bordered three manifold. Lipshitz, Ozsváth and Thurston generalize the hat version of the Ozsváth-Szabó complex for bordered three-manifold by first constructing a graded differential algebra corresponding to the parameterized boundary, and then associating the Bordered Floer modules of type $A$ and $D$ to the bordered manifold, which are respectively an $\mathcal{A}_{\infty}$ module and a module over the differential graded algebra (see LOT1, [LOT2]). Gluing of bordered three-manifolds for constructing closed three-manifolds is translated to an appropriate tensor product construction on the corresponding Bordered Floer modules.

In a different direction, if the boundary of a three-manifold $X$ is decorated with a set $\tau$ of suture, Juhász associates a complex, the so called sutured Floer complex to the sutured manifold $(X, \tau)$ Ju1, provided that $(X, \tau)$ is balanced. The complex generalizes the hat versions of the Ozsváth-Szabó complexes associated with closed three-manifolds and links inside three-manifolds. The theory of sutured manifolds was introduced in Gab1 and developed in Gab2 and Gab3 by D. Gabai in order to study the existence of taut foliations on three-manifolds. Sutured manifolds are oriented three-manifolds with boundary, together with a set of oriented simple closed curves (the sutures) that divide the boundary into positive and negative parts. Gabai defines the so called sutured manifold decomposition which consists of cutting the manifold along a properly embedded oriented surface $R$ and adding one side of $R$ to the plus boundary and the other side to the minus boundary. He shows that a sutured manifold carries a taut foliation if and only if there is a sequence of decompositions that result in a product sutured manifold. Honda, Kazez, and Matic generalized the theory of sutured manifold decomposition for the study of tight contact structures on three-manifolds, and developed the convex decomposition theory HKM1. In addition to the introduction of sutured Floer complex, Juhász described how sutured Floer complex changes through sutured manifold decomposition [Ju2]. As a consequence, he shows that a sutured manifold $(X, \tau)$ is taut if and only if the sutured Floer homology group $\operatorname{SFH}(X, \tau)$ is non-trivial.

These results suggested a deep connection between sutured Floer theory of Juhász and the sutured manifold decomposition theory of Gabai, as well as the contact geometry of three-manifolds. Subsequent developments included the study of sutured Floer polytope by Juhász $\mathrm{Ju} 3$ and introduction of contact invariants for contact three-manifolds with convex boundary by Honda, Kazez and Matić HKM2. This last invariant generalizes the contact invariant of Ozsváth and Szabó for a closed contact three-manifold defined in OS10.

1.3. Outline of the paper. The paper is organized as follows. In section 2 we review some of the basic notions, including the sutured manifolds, the corresponding Heegaard diagrams, and the $\operatorname{Spin}^{c}$ structures on sutured manifolds. We will also review some of the main constructions studied in this paper, including surgery and filling the sutures.

In section 4 we investigate a notion of admissibility for Heegaard diagrams, which makes it possible to construct an Ozsváth and Szabó complex using Heegaard Floer theory. The admissibility condition is slightly weaker, in a sense, than the strong admissibility of Ozsváth and Szabó in the context of closed three-manifolds. However, it is strong enough for the construction of Ozsváth-Szabó complex to work. 
We show that all balanced sutured manifolds admit admissible Heegaard diagrams corresponding to any $\operatorname{Spin}^{c}$ class.

In section 3 we develop the language of chain complexes filtered by a module, and make some simple algebraic observations. Moreover, we construct an algebra associated with the boundary of a balanced sutured manifold, as well as a filtration of its generators by classes in $\mathrm{H}^{2}(X, \partial X ; \mathbb{Z})$. The algebra plays the role of the coefficient ring for the Ozsváth-Szabó chain complex associated with the balanced sutured manifold.

In section 5 we study the orientability issues for the corresponding moduli spaces. In particular, an appropriate orientation for the moduli spaces of boundary degenerations is required so that the differential $\partial$ of the associated Ozsváth-Szabó chain complex satisfies $\partial^{2}=0$. Analyzing the analytic aspects of the theory thus requires some new techniques which are developed in section 5 .

In section 5 we construct the chain complex associated with an admissible Heegaard diagram for the balanced sutured manifold $(X, \tau)$. We show that the filtered chain homotopy type of this complex is invariant under Heegaard moves, and is independent of the choice of the path of almost structure on the symmetric product of the Heegaard surface. The choice of the algebra associated with the boundary plays a very crucial role both in defining the chain complex and proving the invariance of the filtered chain homotopy type.

In section 7 we study how the filtered chain homotopy type of the Ozsváth-Szabó complex associated with a balanced sutured manifold $(X, \tau)$ changes when we add two parallel copies of an existing suture to the boundary with appropriate orientation. The operation is called the stabilization of the sutured manifold $(X, \tau)$. When $(X, \tau)$ corresponds to a knot $K$ inside a closed three-manifold $Y$, the stabilization corresponds to considering multi-pointed Heegaard diagrams for defining the knot Floer complex, and the stabilization formula generalizes the relation between usual Ozsváth-Szabó complexes and the multi-pointed ones.

Finally, in section 8 we introduce a generalization of the surgery triangle for balanced sutured manifolds. The freedom to choose many marked points on the Heegaard diagram allows us to understand the chain maps in a better way, and refine the existing triangles, and long exact sequences. 


\section{BACKGROUND ON SUTURED MANIFOLDS}

2.1. Sutured manifolds and relative $\operatorname{Spin}^{c}$ structures. In this paper, we deal only with balanced sutured manifold, so we will modify the standard definition of sutured manifolds, by throwing away the possibility of having a torus component in the suture.

Definition 2.1. A sutured manifold $(X, \tau)$ is a compact oriented three-manifold $X$ with boundary $\partial X$, together with a set of disjoint oriented simple closed curves $\tau=\left\{\gamma_{1}, \ldots, \gamma_{\kappa}\right\}$ on $\partial X$. We will denote by $A\left(\gamma_{i}\right)$ a tubular neighborhood of $\gamma_{i}$ in $\partial X$, which will be an annulus. We let $A(\tau)=A\left(\gamma_{1}\right) \cup \ldots \cup A\left(\gamma_{\kappa}\right)$. Every component of $\mathfrak{R}(\tau)=\partial X-A(\tau)^{\circ}$ is oriented (where $A(\tau)^{\circ}$ denotes the interior of $A(\tau)$. Let $\mathfrak{R}(\tau)=\mathfrak{R}^{+}(\tau) \cup \mathfrak{R}^{-}(\tau)$ where $\mathfrak{R}^{+}(\tau)$ denotes the union of components of $\mathfrak{R}(\tau)$ with the property that the orientation induced on $\tau$ as the boundary of $\mathfrak{R}^{+}(\tau)$ agrees with the orientation of $\tau$, while $\mathfrak{R}^{-}(\tau)$ denotes the union of components of $\mathfrak{R}(\tau)$ with the property that the orientation induced on $\tau$ as the boundary of $\mathfrak{R}^{-}(\tau)$ is the opposite of the orientation of $\tau$. We assume that the orientation on the components of $\mathfrak{R}(\tau)$ is compatible with the orientation of the boundary $\partial \mathfrak{R}(\tau)$ induced by the sutures $\gamma_{1}, \ldots, \gamma_{\kappa}$. A sutured manifold $(X, \tau)$ is called balanced if $X$ has no closed components, $\chi\left(\mathfrak{R}^{+}(\tau)\right)=\chi\left(\mathfrak{R}^{-}(\tau)\right)$ and the induced map $\pi_{0}(A(\tau)) \rightarrow \pi_{0}(\partial X)$ is surjective.

Definition 2.2. A Heegaard diagram is a tuple $(\Sigma, \boldsymbol{\alpha}, \boldsymbol{\beta}, \mathbf{z})$ such that $(\Sigma, \boldsymbol{\alpha}, \boldsymbol{\beta})$ is a balanced Heegaard diagram i.e. $\Sigma$ is a compact oriented surface and $\boldsymbol{\alpha}$ and $\boldsymbol{\beta}$ are sets of disjoint oriented simple closed curves on $\Sigma$ where $|\boldsymbol{\alpha}|=|\boldsymbol{\beta}|=\ell$, and

$$
\mathbf{z}=\left\{z_{1}, \ldots, z_{\kappa}\right\} \subset \operatorname{int}(\Sigma-\bigcup \boldsymbol{\alpha}-\bigcup \boldsymbol{\beta})
$$

is a set of marked points such that each connected component of $\Sigma-\boldsymbol{\alpha}$ and $\Sigma-\boldsymbol{\beta}$ contains at least one marked point.

Every Heegaard diagram $(\Sigma, \boldsymbol{\alpha}, \boldsymbol{\beta}, \mathbf{z})$ uniquely defines a balanced sutured manifold manifold as follows. Let $\Sigma^{\circ}=\Sigma-D_{1}-\ldots-D_{\kappa}$ denote the complement of small disks $D_{1}, \ldots, D_{\kappa}$ around $z_{1}, \ldots, z_{\kappa}$, where $\mathbf{z}=\left\{z_{1}, \ldots, z_{\kappa}\right\}$. The three-manifold $X$ is obtained from $\Sigma^{\circ} \times[-1,1]$ by attaching 3-dimensional 2-handles along the curves $\alpha_{i} \times\{-1\}$ and $\beta_{j} \times\{1\}$ for $\left.i, j=1, \ldots, \ell\right)$. We may define the set of sutures on the boundary of $X$ by

$$
\tau=\left\{\gamma_{1}, \ldots, \gamma_{\kappa}\right\}, \quad \gamma_{i}=\partial D_{i} \times\{0\} .
$$

In this situation, we say that $(\Sigma, \boldsymbol{\alpha}, \boldsymbol{\beta}, \mathbf{z})$ is associated with the the sutured threemanifold $(X, \tau)$.

Proposition 2.3. For every balanced sutured manifold $(X, \tau)$, there exists a Heegaard diagram associated with it in the above sense.

Proof. Let $\left(\Sigma_{\tau}, \boldsymbol{\alpha}, \boldsymbol{\beta}\right)$ be a sutured Heegaard diagram for the balanced sutured manifold $(X, \tau)$ in the sense of Ju1. If $\tau=\left\{\gamma_{1}, \ldots, \gamma_{\kappa}\right\}$ consists of $\kappa$ sutures, take $\Sigma$ to be the surface obtained from $\Sigma_{\tau}$ by gluing $\kappa$ disks $D_{1}, D_{2}, \ldots, D_{\kappa}$ to it along the boundary components corresponding to $\gamma_{1}, \ldots, \gamma_{\kappa}$. Let $z_{i}$ be the center of $D_{i}$, $i=1, \ldots, \kappa$. Then $\left(\Sigma, \boldsymbol{\alpha}, \boldsymbol{\beta}, \mathbf{z}=\left\{z_{1}, \ldots, z_{\kappa}\right\}\right)$ is a Heegaard diagram for $(X, \tau)$. 
Proposition 2.4. If $\left(\Sigma_{1}, \boldsymbol{\alpha}_{1}, \boldsymbol{\beta}_{1}, \mathbf{z}\right)$ and $\left(\Sigma_{2}, \boldsymbol{\alpha}_{2}, \boldsymbol{\beta}_{2}, \mathbf{w}\right)$ are two Heegaard diagrams for a balanced sutured manifold $(X, \tau)$, then they are diffeomorphic after a finite set of Heegaard moves, which are supported away from the marked points.

Proof. This is proposition 2.15 from [Ju1].

For the most part of this paper, we will identify $\mathfrak{R}(\tau)=\mathfrak{R}^{+}(\tau) \cup \mathfrak{R}^{-}(\tau)$ as the connected components of $\partial X-\tau$. Thus the boundary of each connected component $R \subset \mathfrak{R}(\tau)$ may be identified as a union of curves in $\tau$. In the few situations where the annuli $A\left(\gamma_{i}\right)$ are relevant, we will emphasize them in the notation.

Suppose that $(X, \tau)$ is a balanced sutured manifold. One may define a nowhere vanishing vector field on $\partial X$ as follows. Let $v_{\tau}$ be a vector field (with values in $\left.\left.T X\right|_{\partial X}\right)$ which points outward on $\mathfrak{R}^{+}(\tau) \subset \partial X-A(\tau)=\mathfrak{R}(\tau)$, and points inward on $\mathfrak{R}^{-}(\tau) \subset \mathfrak{R}(\tau)$. Furthermore, under the identification $A\left(\gamma_{i}\right)=\gamma_{i} \times$ $[-1,1]$, let $\left.v_{\tau}\right|_{A\left(\gamma_{i}\right)}$ be the vector field $\frac{\partial}{\partial t}$ determining the unit tangent vector of the second factor, i.e. the interval $[-1,1]$. In fact, we have to perturb $v_{\tau}$ on a small neighborhood $\partial A(\tau)$ to make it continuous, but we typically drop this perturbation from our notation.

Definition 2.5. Suppose that the non-vanishing vector fields $v$ and $w$ on $X$ agree with $v_{\tau}$ on $\partial X$. We say that $v$ and $w$ are homologous if there is a ball $B \subset X^{\circ}$ such that the restrictions of $v$ and $w$ to $X-B$ are homotopic relative the boundary of $X$. We define the space $\operatorname{Spin}^{c}(X, \tau)$ of relative $\operatorname{Spin}^{c}$ structures on the sutured manifold $(X, \tau)$ to be the space of homology classes of such nowhere vanishing vector fields on $X$ which agree with $v_{\tau}$ on $\partial X$.

Note that $\operatorname{Spin}^{c}(X, \tau)$ is an affine space over $\mathrm{H}^{2}(X, \partial X, \mathbb{Z})$. Let us assume that the $\operatorname{Spin}^{c}$ structure $\underline{\mathfrak{s}} \in \operatorname{Spin}^{c}(X, \tau)$ is represented by a nowhere vanishing vector field $v$, so that $\left.v\right|_{\partial X}=v_{\tau}$. Let us define the first Chern class of $\mathfrak{s}$ to be the first Chern class of the oriented 2-plane field $v^{\perp}$ over $X$, which lives in $\mathrm{H}^{2}(X, \mathbb{Z})$. Let us denote the inclusion of $\partial X$ in $X$ by $i: \partial X \rightarrow X$. We thus get a map

$$
i^{*}: \mathrm{H}^{2}(X, \mathbb{Z}) \rightarrow \mathrm{H}^{2}(\partial X, \mathbb{Z}) .
$$

The first Chern class of the 2-plane field $v_{\tau}^{\perp}$ lives in $\mathrm{H}^{2}(\partial X, \mathbb{Z})$ and $c_{1}(\underline{\mathfrak{s}})$ is thus included in

$$
\left(i^{*}\right)^{-1}\left(c_{1}\left(v_{\tau}^{\perp}\right)\right) \subset \mathrm{H}^{2}(X, \mathbb{Z}) .
$$

We may glue a solid cylinder $D^{2} \times[-1,1]$ to each component $A\left(\gamma_{i}\right)$ of $A(\tau)$ along $S^{1} \times[-1,1]$. This way, we obtain a three-manifold with boundary, which will be denoted by $\bar{X}=\bar{X}^{\tau}$. The set of homology classes of no-where vanishing vector fields on $\bar{X}$ which point outward on the positive boundary components of $\bar{X}$ and point inward on the negative boundary components of $\bar{X}$ will be denoted by $\operatorname{Spin}^{c}(\bar{X})$. Note that $\operatorname{Spin}^{c}(\bar{X})$ is an affine space over $\mathrm{H}^{2}(\bar{X}, \partial \bar{X})$. Again, if $\mathfrak{s} \in \operatorname{Spin}^{c}(\bar{X})$ is a given $\operatorname{Spin}^{c}$ structure represented by a nowhere vanishing vector field $w$ as above, we have the notion of the first Chern class associated with $\mathfrak{s}$, which is defined to be $c_{1}\left(w^{\perp}\right)$. Clearly, $c_{1}(\mathfrak{s})$, as defined, is an element of $\mathrm{H}^{2}(\bar{X}, \mathbb{Z})$.

There is a notion of restricting $\operatorname{Spin}^{c}$ structures from $(X, \tau)$ to $\bar{X}$ as follows. If $\underline{\mathfrak{s}} \in \operatorname{Spin}^{c}(X, \tau)$ is represented by the no-where vanishing vector field $v$ (so that 
$\left.v\right|_{\partial X}=v_{\tau}$ ), we may extend $v$ over each one of the glued cylinders $D^{2} \times[-1,1]$. In fact, $v$ may be extended over $D^{2} \times[-1,1]$ by setting it equal to $\frac{\partial}{\partial t}$, where $t$ denotes the variable associated with the interval $[-1,1]$. The new vector field $\bar{v}$ determines

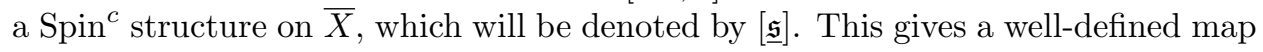

$$
[.]: \operatorname{Spin}^{c}(X, \tau) \longrightarrow \operatorname{Spin}^{c}(\bar{X}) \text {. }
$$

Let us denote the inclusion of $X$ in $\bar{X}$ by $\imath: X \rightarrow \bar{X}$. This inclusion gives a map

$$
\imath^{*}: \mathrm{H}^{2}(\bar{X}, \mathbb{Z}) \longrightarrow \mathrm{H}^{2}(X, \mathbb{Z})
$$

From the definition of the first Chern class, we know that if $[\mathfrak{g}]$ is represented by $\bar{v}$, $\underline{\mathfrak{s}}$ is represented by $v=\imath^{*}(\bar{v})$, and thus

$$
\imath^{*}\left(c_{1}([\mathfrak{\mathfrak { g }}])\right)=c_{1}(\underline{\mathfrak{s}}) .
$$

2.2. Associated sutured manifolds; surgery and filling the sutures. Let $(X, \tau)$ be a balanced sutured manifold, with $\tau$ the set of sutures on the boundary of $X$, and with $\mathfrak{R}^{+}(\tau)$ and $\mathfrak{R}^{-}(\tau)$ the union of positive, respectively negative, components in $\partial X-\tau$ as above. Suppose that $R^{+}$is a component of $\mathfrak{R}^{+}(\tau), R^{-}$ is a component of $\mathfrak{R}^{-}(\tau)$, and $\gamma_{1}$ and $\gamma_{2}$ are sutures in $\tau$ such that

$$
\partial R^{+} \cap \partial R^{-}=\left\{\gamma_{1}, \gamma_{2}\right\} .
$$

Let $\lambda$ be a simple closed curve, which consists of a union $\lambda=\lambda^{+} \cup \lambda^{-}$, with $\lambda^{\bullet} \subset \overline{R^{\bullet}}, \bullet \in\{+,-\}$, which cuts either of $\gamma_{1}$ and $\gamma_{2}$ in a single transverse point. Sometimes we may assume that the image of the homology class $[\lambda] \in \mathrm{H}_{1}(\partial X, \mathbb{Z})$ in $\mathrm{H}_{1}(X, \mathbb{Z})$ is trivial, i.e. that $\lambda$ bounds a closed surface in $X$. In this situation, we will say that $\lambda$ is homologically trivial in $X$. It makes sense to talk about Morse surgery along $\lambda$ on the sutured manifold $X$ as will follow.

Consider two parallel copies of $\lambda$ which we will denote by

$$
\lambda_{1}=\lambda_{1}^{+} \cup \lambda_{1}^{-}, \quad \& \quad \lambda_{2}=\lambda_{2}^{+} \cup \lambda_{2}^{-} .
$$

Let $N_{i}$ be a tubular neighborhood of $\gamma_{i}$ in $\partial X$, which may be identified with the standard cylinder $N_{i}=S^{1} \times I$, where $I$ is the unit interval. The pair of arcs $\left(\lambda_{1} \cup \lambda_{2}\right) \cap N_{i}$ may then be pictured as $\{1,-1\} \times I$, where the area between the parallel curves $\lambda_{1}$ and $\lambda_{2}$ is identified as

$$
\left\{z \in S^{1} \mid \operatorname{Im}(z)>0\right\} \times I \subset S^{1} \times I=N_{i} .
$$

We may change the arcs $\{1,-1\} \times I$ with the arcs

$$
\begin{aligned}
& \left\{\left(e^{t \pi \sqrt{-1}}, t\right) \mid t \in I=[0,1]\right\} \subset S^{1} \times I, \quad \& \\
& \left\{\left(-e^{t \pi \sqrt{-1}}, t\right) \mid t \in I=[0,1]\right\} \subset S^{1} \times I
\end{aligned}
$$

in both $N_{1}$ and $N_{2}$. With this change, the curves $\lambda_{1}$ and $\lambda_{2}$ are replaced by new simple closed curves $\delta_{1}$ and $\delta_{2}$. Let $\left(X, \tau_{\lambda}\right)$ be the balanced sutured manifold defined with

$$
\tau_{\lambda}=\left(\tau-\left\{\gamma_{1}, \gamma_{2}\right\}\right) \cup\left\{\delta_{1}, \delta_{2}\right\} .
$$

In order to see that $\left(X, \tau_{\lambda}\right)$ is balanced, it suffices to note that

$$
\partial X-\tau_{\lambda}=\left((\partial X-\tau)-\left(R^{+} \cup R^{-}\right)\right) \cup\left(S^{+} \cup S^{-}\right),
$$

where

$$
S^{+}=\left(R^{+}-\operatorname{nd}\left(\lambda^{+}\right)\right) \cup \operatorname{nd}\left(\lambda^{-}\right), \quad \& \quad S^{-}=\left(R^{-}-\operatorname{nd}\left(\lambda^{-}\right)\right) \cup \operatorname{nd}\left(\lambda^{+}\right),
$$


and thus $\chi\left(S^{\bullet}\right)=\chi\left(R^{\bullet}\right), \bullet \in\{+,-\}$. The surgery is usually denoted by $(X, \tau) \rightsquigarrow$ $\left(X, \tau_{\lambda}\right)$ in this paper.

Suppose that the simple closed curve $\lambda$ is chosen as above. For $n=\left(n_{1}, n_{2}\right)$ in $\mathbb{Z} \oplus \mathbb{Z}$, let $\lambda(n)$ be the simple closed curve obtained from $\lambda$ by winding it $n_{1}$ times around $\gamma_{1}$ (close to the intersection of $\lambda$ with $\gamma_{1}$ ) and $n_{2}$ times around $\gamma_{2}$ (close to the intersection of $\lambda$ with $\gamma_{2}$ ). The homology class represented by $\lambda(n)$ in $\mathrm{H}_{1}(\partial X, \mathbb{Z})$ is $[\lambda]+n_{1}\left[\gamma_{1}\right]-n_{2}\left[\gamma_{2}\right]$. When there is no confusion, we will denote $\left(X, \tau_{\lambda(n)}\right)$ by $\left(X, \tau_{n}\right)$.

Let us assume that

$$
H=\left(\Sigma, \boldsymbol{\alpha}=\left\{\alpha_{1}, \ldots, \alpha_{\ell}\right\}, \boldsymbol{\beta}=\left\{\beta_{1}, \ldots, \beta_{\ell}\right\}, \mathbf{z}=\left\{z_{1}, \ldots, z_{\kappa}\right\}\right)
$$

is a Heegaard diagram for the sutured manifold $(X, \tau)$. Here $\Sigma$ is a closed Riemann surface of genus $g, \boldsymbol{\alpha}$ and $\boldsymbol{\beta}$ are $\ell$-tuples of simple closed curves which are homologically linearly independent in $\Sigma-\mathbf{z}$, and $\mathbf{z}=\left\{z_{1}, \ldots, z_{\kappa}\right\}$ is a $\kappa$-tuple of marked points determining the set $\tau$ of sutures on the boundary of the three-manifold $X$. Furthermore, let us assume that $z_{1}, z_{2}$ are the marked points corresponding to the sutures $\gamma_{1}$ and $\gamma_{2}$ respectively. We may choose the Heegaard diagram so that $z_{1}$ and $z_{2}$ are in the same connected component of $\Sigma-\boldsymbol{\alpha}-\boldsymbol{\beta}_{0}$, where $\boldsymbol{\beta}_{0}$ denotes the subset $\boldsymbol{\beta}-\left\{\beta_{\ell}\right\}$ of $\boldsymbol{\beta}$. In this diagram, the framing $\lambda$ is determined as an arc which joins $z_{1}$ to $z_{2}$ in $\Sigma-\boldsymbol{\beta}$, and may be completed to a simple closed curve by adding to it a short arc from $z_{2}$ to $z_{1}$, meeting $\beta_{\ell}$ transversely in a single point, and staying disjoint from $\boldsymbol{\alpha} \cup \boldsymbol{\beta}_{0}$.

The Heegaard diagram describing the surgery $(X, \tau) \rightsquigarrow\left(X, \tau_{\lambda}\right)$ is then obtained as follows. Let

$$
H_{\lambda}=\left(\Sigma, \boldsymbol{\alpha}, \boldsymbol{\beta}_{\lambda}=\left\{\beta_{1}^{\prime}, \ldots, \beta_{\ell}^{\prime}\right\}, \mathbf{z}\right),
$$

where $\beta_{i}^{\prime}$ for $i=1, \ldots, \ell-1$ is an exact Hamiltonian isotope of the curve $\beta_{i}$ which cuts it in a pair of canceling transverse intersection points. Moreover, $\beta_{\ell}^{\prime}$ is obtained as an isotope of the simple closed curve on $\Sigma$ associated with $\lambda$ which separates the marked points $z_{1}$ and $z_{2}$ from each other. Abusing the notation, we will sometimes denote $\beta_{\ell}^{\prime}$ by $\lambda$. This Heegaard diagram corresponds to the balanced sutured manifold $\left(X, \tau_{\lambda}\right)$. The Heegaard triple

$$
\left(\Sigma, \boldsymbol{\alpha}, \boldsymbol{\beta}, \boldsymbol{\beta}_{\lambda}, \mathbf{z}\right)
$$

will then described the surgery $(X, \tau) \rightsquigarrow\left(X, \tau_{\lambda}\right)$ associated with $\lambda$.

We are particularly interested in the case where the curves $\gamma_{1}$ and $\gamma_{2}$ in $\tau=$ $\left\{\gamma_{1}, \ldots, \gamma_{\kappa}\right\}$ used for the surgery are the boundary of a a subset $B \subset \partial X$ which is homeomorphic to a cylinder $[0,1] \times S^{1}$ (and $\gamma_{1}$ and $\gamma_{2}$ are identified with $\{0\} \times S^{1}$ and $\{1\} \times S^{1}$ respectively). Then for $n=\left(n_{1}, n_{2}\right)$ and $m=\left(m_{1}, m_{2}\right)$ in $\mathbb{Z} \oplus \mathbb{Z}$, the sutured manifolds $\left(X, \tau_{n}\right)$ and $\left(X, \tau_{m}\right)$ are the same if $m_{1}+m_{2}=n_{1}+n_{2}$. Thus in this case, it makes sense to talk about $\left(X, \tau_{n}\right)$ for $n \in \mathbb{Z}$, if the simple closed curve $\lambda$ is fixed.

Let $(X, \tau)$ be a balanced sutured manifold as above. Let $I$ denote a subset of $\{1, \ldots, \kappa\}$. Consider the sutured manifold $(X(I), \tau(I))$ obtained by filling out the sutures of $(X, \tau)$ corresponding to the subset $I$ with solid cylinders $D^{2} \times[-1,1]$. 
In particular, we have $\bar{X}=X(1, \ldots, \kappa)$. In terms of the Heegaard diagrams, if $\left(\Sigma, \boldsymbol{\alpha}, \boldsymbol{\beta}, \mathbf{z}=\left\{z_{1}, \ldots, z_{\kappa}\right\}\right)$ is a Heegaard diagram associated with $(X, \tau)$ so that $z_{i}$ corresponds to the suture $\gamma_{i}$, a diagram for $(X(I), \tau(I))$ will be the pointed Heegaard diagram

$$
\left(\Sigma, \boldsymbol{\alpha}, \boldsymbol{\beta}, \mathbf{z}-\left\{z_{i} \mid i \in I\right\}\right) .
$$

Let $(\Sigma, \boldsymbol{\alpha}, \boldsymbol{\beta}, \mathbf{z})$ be a Heegaard diagram for the balanced sutured manifold $(X, \tau)$. Consider the symmetric product

$$
\operatorname{Sym}^{\ell}(\Sigma)=\frac{\Sigma^{\times \ell}}{S_{\ell}}=\frac{\Sigma \times \ldots \times \Sigma}{S_{\ell}}
$$

equipped with a path of complex structures of the form $\left\{J_{t}=\operatorname{Sym}^{\ell}\left(j_{t}\right)\right\}_{t \in[0,1]}$, which is induced from a path of complex structure $\left\{j_{t}\right\}_{t \in[0,1]}$ on $\Sigma$, such that the map $\Sigma^{\times \ell} \rightarrow \operatorname{Sym}^{\ell}(\Sigma)$ is $\left(j_{t}, J_{t}\right)$-holomorphic for all $t \in[0,1]$. Then $\mathbb{T}_{\alpha}=\alpha_{1} \times \ldots \times \alpha_{\ell}$ and $\mathbb{T}_{\beta}=\beta_{1} \times \ldots \times \beta_{\ell}$ are totally real sub-manifolds of $\operatorname{Sym}^{\ell}(\Sigma)$. We may define a map

$$
\underline{\mathfrak{s}}=\underline{\mathfrak{s}}_{\mathbf{z}}: \mathbb{T}_{\alpha} \cap \mathbb{T}_{\beta} \longrightarrow \operatorname{Spin}^{c}(X, \tau)
$$

which is defined by choosing a Morse function compatible with the Heegaard diagram for the sutured manifold $(X, \tau)$, viewing an intersection point $\mathbf{x} \in \mathbb{T}_{\alpha} \cap \mathbb{T}_{\beta}$ as a set of flow lines joining index- 1 critical points to index- 2 critical points of the Morse function, and perturbing the gradient vector field of the corresponding Morse function in a neighborhood of this set of flow lines associated with $\mathbf{x}$ in order to obtain a nowhere vanishing vector field on $X$ with the desired properties.

Denote the natural maps obtained by extending the relative $\operatorname{Spin}^{c}$ structures on sutured manifolds over the attached solid cylinders by

$$
s_{I}=s_{I}^{\tau}: \operatorname{Spin}^{c}(X, \tau) \longrightarrow \operatorname{Spin}^{c}(X(I), \tau(I)), \quad \forall I \subset\{1, \ldots, \kappa\} .
$$

In particular, $s_{\{1, \ldots, \kappa\}}^{\tau}$ is the restriction map [.] : $\operatorname{Spin}^{c}(X, \tau) \rightarrow \operatorname{Spin}^{c}(\bar{X})$ defined before. Note that there is an exact sequence

$$
0 \longrightarrow\left\langle\mathrm{PD}\left[\gamma_{i}\right] \mid i \in I\right\rangle_{\mathbb{Z}} \longrightarrow \operatorname{Spin}^{c}(X, \tau) \stackrel{s_{I}}{\longrightarrow} \operatorname{Spin}^{c}(X(I), \tau(I)) \longrightarrow 0 .
$$

This sequence should be interpreted as follows. If two relative $\operatorname{Spin}^{c}$ structures $\underline{\mathfrak{s}}, \underline{\mathfrak{t}} \in \operatorname{Spin}^{c}(X, \tau)$ satisfy $s_{I}(\underline{\mathfrak{s}})=s_{I}(\underline{\mathfrak{t}})$, then the cohomology class $\underline{\mathfrak{s}}-\underline{\mathfrak{t}}$ is generated by the Poincaré duals of the sutures corresponding to $I$.

2.3. Relative $\operatorname{Spin}^{c}$-structures and Heegaard diagrams. Let the Heegaard diagram $(\Sigma, \boldsymbol{\alpha}, \boldsymbol{\beta}, \mathbf{z})$ for the balanced sutured manifold $(X, \tau)$, the symmetric product $\operatorname{Sym}^{\ell}(\Sigma)$, the totally real tori $\mathbb{T}_{\alpha}$ and $\mathbb{T}_{\beta}$, and the path of complex structures $\left\{J_{t}=\operatorname{Sym}^{\ell}\left(j_{t}\right)\right\}_{t \in[0,1]}$, be as before.

Definition 2.6. Let $D \subset \mathbb{C}$ be the unit disk in the complex plane, and $\mathbf{x}, \mathbf{y} \in$ $\mathbb{T}_{\alpha} \cap \mathbb{T}_{\beta}$. A Whitney disk is a continuous map $\phi: D \rightarrow \operatorname{Sym}^{\ell}(\Sigma)$ such that $\phi(-i)=$ $\mathbf{x}, \phi(i)=\mathbf{y}$ and

$$
\begin{aligned}
& \phi\{z \in \partial D \mid \operatorname{Re}(z) \geq 0\} \subset \mathbb{T}_{\alpha} \quad \& \\
& \phi\{z \in \partial D \mid \operatorname{Re}(z) \leq 0\} \subset \mathbb{T}_{\beta} .
\end{aligned}
$$

The set of homotopy classes of Whitney disks connecting $\mathbf{x}$ to $\mathbf{y}$ is denoted by $\pi_{2}(\mathbf{x}, \mathbf{y})$. For any homology class $\phi \in \pi_{2}(\mathbf{x}, \mathbf{y})$, we will denote the moduli space of 
$\left\{J_{t}\right\}_{t}$-holomorphic representatives of $\phi$ by $\mathcal{M}(\phi)$. There exists a translation action of $\mathbb{R}$ on $\mathcal{M}(\phi)$. The quotient of $\mathcal{M}(\phi)$ under this action will be denoted by $\widehat{\mathcal{M}}(\phi)$. The Maslov index of $\phi$ is denoted by $\mu(\phi)$. For $i \in \mathbb{Z}$, we will denote by $\pi_{2}^{i}(\mathbf{x}, \mathbf{y})$ the subset of $\pi_{2}(\mathbf{x}, \mathbf{y})$ which consists of all $\phi$ with $\mu(\phi)=i$.

It is known ( $\mathrm{OS} 5$, and OS8 $)$ that for any generic path $\left\{J_{t}\right\}_{t}$ of complex structures, $\mathcal{M}(\phi)$ is a smooth manifold of dimension $\mu(\phi)$, which is not necessarily compact. In fact, this moduli space may be compactified by adding the Gromov limits of pseudo-holomorphic curves. But the boundary strata which correspond to degenerations of the domain are not necessarily of lower dimension. We will return to this issue in section 5 .

Definition 2.7. Let $\mathcal{D}_{1}, \ldots, \mathcal{D}_{m}$ be the connected components of $\Sigma-\boldsymbol{\alpha}-\boldsymbol{\beta}$. Each element of the free abelian group generated by $\left\{\mathcal{D}_{1}, \ldots, \mathcal{D}_{m}\right\}$ is called a domain. $A$ domain $\mathcal{D}=a_{1} \mathcal{D}_{1}+\ldots+a_{m} \mathcal{D}_{m}$ is called positive, denoted $\mathcal{D} \geq 0$, if $a_{i} \geq 0$ for $1 \leq i \leq m$. It is called periodic if its boundary is a sum of $\alpha$ and $\beta$ curves.

For every Whitney disk $\phi$ connecting intersection points $\mathbf{x}$ and $\mathbf{y}$, the domain associated with $\phi$ is defined as follows:

$$
\mathcal{D}(\phi)=\sum_{i=1}^{m} n_{p_{i}}(\phi) \mathcal{D}_{i}
$$

where $p_{i} \in \mathcal{D}_{i}$ is a marked point. Here $n_{p}(\phi)$ for a point $p \in \Sigma-\boldsymbol{\alpha}-\boldsymbol{\beta}$ denotes the algebraic intersection number of $\phi$ with the subvariety

$$
\Delta_{p}=\left\{\left(p_{1}, \ldots, p_{\ell}\right) \in \operatorname{Sym}^{\ell}(\Sigma) \mid p_{i}=p, \text { for some } 1 \leq i \leq \ell\right\} .
$$

If the map $\phi$ is holomorphic then $\mathcal{D}(\phi)$ is obviously a positive domain. We will denote by $\pi_{2}^{+}(\mathbf{x}, \mathbf{y})$ the subset of $\pi_{2}(\mathbf{x}, \mathbf{y})$ which consists of all $\phi$ with $\mathcal{D}(\phi) \geq 0$.

If $\mathcal{P}$ is a periodic domain we can associate to it a homology class in $H_{2}(\bar{X}, \mathbb{Z})$. More precisely, let

$$
\partial \mathcal{P}=\sum_{i=1}^{\ell} a_{i} \alpha_{i}+\sum_{i=1}^{\ell} b_{i} \beta_{i}
$$

and let $D_{i}$ be the union of $\alpha_{i} \times[-1,0]$ with the core of the two-handles attached to $\alpha_{i} \times\{-1\}$ in $X$. Similarly, let $D_{i}^{\prime}$ be the union of $\beta_{i} \times[0,1]$ with the core of the two-handle attached to $\beta_{i} \times\{1\}$. Define

$$
H(\mathcal{P})=\mathcal{P}+\sum_{i=1}^{\ell} a_{i} D_{i}+\sum_{i=1}^{\ell} b_{i} D_{i}^{\prime}
$$

If $\phi \in \pi_{2}(\mathbf{x}, \mathbf{x})$ is a Whitney disk connecting $\mathbf{x}$ to itself, with $\mathbf{x} \in \mathbb{T}_{\alpha} \cap \mathbb{T}_{\beta}$, the domain $\mathcal{D}(\phi)$ will be a periodic domain. Conversely, any periodic domain $\mathcal{P}$ determines the class of a Whitney disk in $\pi_{2}(\mathbf{x}, \mathbf{x})$ for any $\mathbf{x} \in \mathbb{T}_{\alpha} \cap \mathbb{T}_{\beta}$. Thus the space of periodic domains may be identified with $\pi_{2}(\mathbf{x}, \mathbf{x})$.

For each $\mathbf{x} \in \mathbb{T}_{\alpha} \cap \mathbb{T}_{\beta}$ let $\gamma_{\mathbf{x}}$ be the flow lines of a compatible Morse function connecting the index-1 critical points to the index- 2 critical points passing through the union $\mathbf{x}$ of the intersection points on $\Sigma \times\{0\} \subset X$. 
Lemma 2.8. For $\mathbf{x}, \mathbf{y} \in \mathbb{T}_{\alpha} \cap \mathbb{T}_{\beta}$ we have $\underline{\mathfrak{s}}(\mathbf{x})-\underline{\mathfrak{s}}(\mathbf{y})=\operatorname{PD}(\epsilon(\mathbf{x}, \mathbf{y}))$ where $\epsilon(\mathbf{x}, \mathbf{y})=$ $\gamma_{\mathbf{x}}-\gamma_{\mathbf{y}} \in \mathrm{H}_{1}(X, \mathbb{Z})$.

Proof. This is lemma 4.7 from [Ju1].

Corollary 2.9. If $\phi \in \pi_{2}(\mathbf{x}, \mathbf{y})$ then we have

$$
\underline{\mathfrak{s}}(\mathbf{x})-\underline{\mathfrak{s}}(\mathbf{y})=\sum_{i=1}^{\kappa} n_{z_{i}}(\phi) \cdot \operatorname{PD}\left[\gamma_{i}\right] .
$$

Proof. The disk $\phi$ gives a domain $\mathcal{D}(\phi)$, with the property that $\epsilon(\mathbf{x}, \mathbf{y})$ is represented by

$$
\partial(\mathcal{D}(\phi)) \in \mathrm{H}_{1}(X, \mathbb{Z})=\frac{\mathrm{H}_{1}\left(\Sigma-\left\{z_{1}, \ldots, z_{\kappa}\right\}, \mathbb{Z}\right)}{\left\langle\alpha_{1}, \ldots, \alpha_{\ell}, \beta_{1}, \ldots, \beta_{\ell}\right\rangle_{\mathbb{Z}}} .
$$

If $\epsilon_{i}$ denotes a small loop around $z_{i} \in \Sigma$, the domain $\mathcal{D}(\phi)$ gives a 2-chain connecting $\epsilon(\mathbf{x}, \mathbf{y})$ and $n_{z_{1}}(\phi) \epsilon_{1}+\ldots+n_{z_{\kappa}}(\phi) \epsilon_{\kappa}$. However, $\epsilon_{i}$ is homologous to $\gamma_{i}$, and we thus have

$$
\underline{\mathfrak{s}}(\mathbf{x})-\underline{\mathfrak{s}}(\mathbf{y})=\operatorname{PD}[\epsilon(\mathbf{x}, \mathbf{y})]=\sum_{i=1}^{\kappa} n_{z_{i}}(\phi) \operatorname{PD}\left[\gamma_{i}\right] .
$$

This completes the proof of the corollary.

Let us finish this subsection with a lemma for computing the Maslov index of a periodic domain. Let

$$
\Sigma-\boldsymbol{\alpha}=\bigcup_{i=1}^{k} A_{i}, \quad \& \quad \Sigma-\boldsymbol{\beta}=\bigcup_{i=1}^{l} B_{i},
$$

and assume we have $m=k+l-1$ points $w_{1}, \ldots, w_{m}$ on $\Sigma$ such that $w_{i} \in A_{i} \cap B_{1}$ for $1 \leq i \leq k$, and $w_{i+k} \in A_{k} \cap B_{i+1}$ for $1 \leq i<l$.

Lemma 2.10. For any periodic domain $\mathcal{P} \in \pi_{2}(\mathbf{x}, \mathbf{x})$ such that $n_{w_{i}}(\mathcal{P})=0$ for $1 \leq i \leq m$ we have:

$$
\mu(\mathcal{P})=\left\langle c_{1}([\underline{\mathfrak{s}}(\mathbf{x})]), H(\mathcal{P})\right\rangle .
$$

Proof. Let $\Sigma_{\mathbf{w}}=\Sigma-\mathrm{nd}(\mathbf{w})$, with $\mathbf{w}=\left\{w_{1}, \ldots, w_{m}\right\}$. Now $\left(\Sigma_{\mathbf{w}}, \boldsymbol{\alpha}, \boldsymbol{\beta}\right)$ is a sutured Heegaard diagram for a sutured manifold $X_{\mathbf{w}}$ which is obtained from $\bar{X}$ by removing neighborhoods of the flow lines passing through $\mathbf{w}$. If $i: X_{\mathbf{w}} \rightarrow \bar{X}$ is the embedding of $X_{\mathbf{w}}$ in $\bar{X}$, then $i^{-1}(\mathcal{P})$ is a periodic domain in $\left(\Sigma_{\mathbf{w}}, \boldsymbol{\alpha}, \boldsymbol{\beta}\right)$, and by theorem 5.2 from [Ju1] we have

$$
\mu\left(i^{-1} \mathcal{P}\right)=\left\langle c_{1}\left(\underline{\mathfrak{s}}_{\mathbf{w}}(\mathbf{x})\right), H\left(i^{-1}(\mathcal{P})\right)\right\rangle .
$$

We have $i^{*} H(\mathcal{P})=H\left(i^{-1}(\mathcal{P})\right)$. Thus it is enough to show that

$$
c_{1}\left(\underline{\mathfrak{s}}_{\mathbf{w}}(\mathbf{x})\right)=i^{*} c_{1}([\underline{\mathfrak{g}}(\mathbf{x})]) .
$$

Let $\nu$ be the vector field defining $\mathfrak{s}(\mathbf{x})$, and let $\bar{\nu}$ be the extension of $\nu$ to $\bar{X}$. Then $i^{*} \bar{\nu}$ is the vector field defining $\underline{\mathfrak{s}}_{\mathbf{w}}(\mathbf{x})$ and thus equation 1 is satisfied. 
Lemma 2.11. For any periodic domain $\mathcal{P} \in \pi_{2}(\mathbf{x}, \mathbf{x})$ we have:

$$
\mu(\mathcal{P})=\left\langle c_{1}(\underline{\mathfrak{s}}(\mathbf{x})), H(\mathcal{P})\right\rangle .
$$

Proof. Moving the curves by isotopies does not change the two sides of the above equality. We may thus assume that we have $m=k+l-1$ points $w_{1}, \ldots, w_{m}$ on $\Sigma$ such that $w_{i} \in A_{i} \cap B_{1}$ for $1 \leq i \leq k$, and $w_{i+k} \in A_{k} \cap B_{i+1}$ for $1 \leq i<l$. Let us denote $n_{w_{i}}(\mathcal{P})$ by $n_{i}$, and set

$$
\mathcal{Q}=\mathcal{P}-\left(\sum_{i=1}^{k} n_{i} A_{i}\right)-\left(\sum_{i=1}^{l-1}\left(n_{i+k}-n_{k}\right) B_{i+1}\right)
$$

Clearly $n_{w_{i}}(\mathcal{Q})=0$ for $i=1, \ldots, m$, and lemma 2.10 implies (setting $\mathfrak{s}=[\mathfrak{s}(\mathbf{x})]$, and regarding $\mathcal{Q}$ as an element in $\left.\pi_{2}(\mathbf{x}, \mathbf{x})\right)$

$$
\begin{aligned}
\mu(\mathcal{Q})= & \left\langle c_{1}(\mathfrak{s}), H(\mathcal{Q})\right\rangle \\
= & \left\langle c_{1}(\mathfrak{s}), H(\mathcal{P})\right\rangle-\left(\sum_{i=1}^{k} n_{i}\left\langle c_{1}(\mathfrak{s}), H\left(A_{i}\right)\right\rangle\right) \\
& \quad-\left(\sum_{i=1}^{l-1}\left(n_{i+k}-n_{k}\right)\left\langle c_{1}(\mathfrak{s}), H\left(B_{i+1}\right)\right\rangle\right) \\
= & \left\langle c_{1}(\mathfrak{s}), H(\mathcal{P})\right\rangle-\left(\sum_{i=1}^{k} n_{i} \chi\left(A_{i}\right)\right)-\left(\sum_{i=1}^{l-1}\left(n_{i+k}-n_{k}\right) \chi\left(B_{i}\right)\right) .
\end{aligned}
$$

In the last equation we denote by $\chi\left(A_{i}\right)$ and $\chi\left(B_{i}\right)$ the expressions $2-2 g_{A_{i}}$ and $2-2 g_{B_{i}}$, respectively, where $g_{A_{i}}$ and $g_{B_{i}}$ denote the genera of the components in $\mathfrak{R}^{-}(\tau)$ and $\mathfrak{R}^{+}(\tau)$ which correspond to $A_{i}$ and $B_{i}$, respectively.

On the other hand, the formula of Lipshitz (Lip) may be used to compute $\mu\left(A_{i}\right)$ and $\mu\left(B_{j}\right)$ as periodic domains in $\pi_{2}(\mathbf{x}, \mathbf{x})$. As such, we will have

$$
\mu\left(A_{i}\right)=\chi\left(A_{i}\right), \quad i=1, \ldots, k, \quad \& \mu\left(B_{j}\right)=\chi\left(B_{i}\right), \quad j=1, \ldots, l .
$$

Combining equations 3 and 2 we obtain

$$
\begin{aligned}
\mu(\mathcal{P})=\left\langle c_{1}(\mathfrak{s}), H(\mathcal{P})\right\rangle & -\left(\sum_{i=1}^{k} n_{i} \chi\left(A_{i}\right)\right)-\left(\sum_{i=1}^{l-1}\left(n_{i+k}-n_{k}\right) \chi\left(B_{i}\right)\right) \\
& +\left(\sum_{i=1}^{k} n_{i} \mu\left(A_{i}\right)\right)+\left(\sum_{i=1}^{l-1}\left(n_{i+k}-n_{k}\right) \mu\left(B_{i}\right)\right) \\
=\left\langle c_{1}(\mathfrak{s}), H(\mathcal{P})\right\rangle . &
\end{aligned}
$$

This completes the proof of the lemma. 


\section{Algebra inPut}

3.1. The $\mathbb{A}$-chain complexes. Let us assume that $\mathbb{A}$ is a (commutative) finitely generated $\mathbb{Z}$-algebra.

Definition 3.1. If $\mathbb{B}$ is another (commutative) ring, together with a homomorphism $\phi_{\mathbb{B}}: \mathbb{A} \rightarrow \mathbb{B}$ we will call $\left(\mathbb{B}, \phi_{\mathbb{B}}\right)$ a test ring for $\mathbb{A}$.

In other words, a test ring is a ring that has the structure of an $\mathbb{A}$-module. We may then define $\phi_{\mathbb{B}}(a)=a .1_{\mathbb{B}}$ for $a \in \mathbb{A}$. We will denote the image of an element $a \in \mathbb{A}$ in $\mathbb{B}$ by $[a]^{\mathbb{B}}$, and drop $\phi_{\mathbb{B}}$ from the notation for simplicity.

Definition 3.2. A chain complex with coefficient ring $\mathbb{A}$, or simply an $\mathbb{A}$ chain complex, is an $\mathbb{A}$-module $C$, together with a homomorphism of $\mathbb{A}$-modules $d: C \rightarrow$ $C$, such that $d \circ d=0$.

Let us assume that $(C, d)$ is an $\mathbb{A}$ chain complex. Choose a test ring $\mathbb{B}$ and let $C(\mathbb{B})=C \otimes_{\mathbb{A}} \mathbb{B}$. The differential $d$ of the complex $(C, d)$ induces a differential $d^{\mathbb{B}}: C(\mathbb{B}) \rightarrow C(\mathbb{B})$.

Definition 3.3. If $\left(C_{1}, d_{1}\right)$ and $\left(C_{2}, d_{2}\right)$ are $\mathbb{A}$ chain complexes, a homomorphism $f: C_{1} \rightarrow C_{2}$ of $\mathbb{A}$-modules is called an $\mathbb{A}$ chain map if $f \circ d_{1}=d_{2} \circ f$.

The following lemma is an immediate consequence of the definitions.

Lemma 3.4. Let $\mathbb{A}$ be as above and suppose that $\mathbb{B}$ is a test ring for $\mathbb{A}$. If $\left(C_{1}, d_{1}\right)$ and $\left(C_{2}, d_{2}\right)$ are $\mathbb{A}$ chain complexes and $f: C_{1} \rightarrow C_{2}$ is an $\mathbb{A}$ chain map, then $f$ induces a $\mathbb{B}$ chain map

$$
f^{\mathbb{B}}:\left(C_{1}(\mathbb{B}), d_{1}^{\mathbb{B}}\right) \longrightarrow\left(C_{2}(\mathbb{B}), d_{2}^{\mathbb{B}}\right)
$$

where $d_{i}^{\mathbb{B}}$ denotes the differential induced by $d_{i}$ on $C_{i}(\mathbb{B}), i=1,2$.

Associated with any $\mathbb{A}$-chain complex $(C, d)$, and any test ring $\mathbb{B}$, we consider the homology group

$$
H_{*}(C, d ; \mathbb{B}):=H_{*}\left(C(\mathbb{B}), d^{\mathbb{B}}\right) .
$$

We may denote this homology group by $H_{*}(C ; \mathbb{B})$, if there is no confusion. If $\left(C_{1}, d_{1}\right)$ and $\left(C_{2}, d_{2}\right)$ are $\mathbb{A}$ chain complexes and $f: C_{1} \rightarrow C_{2}$ is an $\mathbb{A}$ chain map, then the above lemma implies that $f$ induces a homomorphism

$$
f_{*}^{\mathbb{B}}: H\left(C_{1}, d_{1} ; \mathbb{B}\right) \longrightarrow H\left(C_{2}, d_{2} ; \mathbb{B}\right) .
$$

Definition 3.5. An $\mathbb{A}$-chain map $f:\left(C_{1}, d_{1}\right) \rightarrow\left(C_{2}, d_{2}\right)$ between $\mathbb{A}$ chain complexes is called null-homotopic if there is another $\mathbb{A}$ chain map $H:\left(C_{1}, d_{1}\right) \rightarrow$ $\left(C_{2}, d_{2}\right)$ such that $f=H \circ d_{1}-d_{2} \circ H . f$ is called a homotopy equivalence of $\mathbb{A}$ chain complexes if there exist an $\mathbb{A}$-chain map $g:\left(C_{2}, d_{2}\right) \rightarrow\left(C_{1}, d_{1}\right)$ such that $g \circ f-I d_{C_{1}}$ and $f \circ g-I d_{C_{2}}$ are null-homotopic. $f:\left(C_{1}, d_{1}\right) \rightarrow\left(C_{2}, d_{2}\right)$ is called $a$ quasi-isomorphism if the induced map

$$
f_{*}^{\mathbb{B}}: H\left(C_{1}, d_{1} ; \mathbb{B}\right) \longrightarrow H\left(C_{2}, d_{2} ; \mathbb{B}\right)
$$

is an isomorphism for any test ring $\mathbb{B}$. More generally, if $\mathfrak{B}$ is a family of test rings for $\mathbb{A}$, the $\mathbb{A}$ chain map $f$ is called a $\mathfrak{B}$-isomorphism if $f_{*}^{\mathbb{B}}$ is an isomorphism for any test ring $\mathbb{B} \in \mathfrak{B}$. Two $\mathbb{A}$ chain complexes $\left(C_{1}, d_{1}\right)$ and $\left(C_{2}, d_{2}\right)$ are quasi-isomorphic if there is a third $\mathbb{A}$ chain complex $(C, d)$, together with quasi-isomorphisms $f_{i}$ : $\left(C_{i}, d_{i}\right) \rightarrow(C, d), i=1,2$. Similarly, we may define $\mathfrak{B}$-isomorphic $\mathbb{A}$ chain complexes. 
Lemma 3.6. If $f:\left(C_{1}, d_{1}\right) \rightarrow\left(C_{2}, d_{2}\right)$ is a homotopy equivalence of $\mathbb{A}$ chain complexes, then $f$ is a quasi-isomorphism.

Proof. If $g:\left(C_{2}, d_{2}\right) \rightarrow\left(C_{1}, d_{1}\right)$ is the inverse of $f$ such that

$$
g \circ f-I d_{C_{1}}=H \circ d_{1}-d_{1} \circ H, \quad \& \quad f \circ g-I d_{C_{2}}=K \circ d_{2}-d_{2} \circ K,
$$

for homotopy maps $H$ and $K$, we obtain the induced maps $f^{\mathbb{B}}, g^{\mathbb{B}}, H^{\mathbb{B}}$ and $K^{\mathbb{B}}$ over the induced complexes associated with any test ring $\mathbb{B}$. Thus, $f_{*}^{\mathbb{B}}$ is an isomorphism for any test ring $\mathbb{B}$, and $g_{*}^{\mathbb{B}}$ is its inverse.

3.2. The mapping cones of $\mathbb{A}$-chain maps. Most part of this sub-section is borrowed from Ozsváth and Szabó's [S6] (subsection 4.1) with minor modifications.

If $\left(A_{1}, d_{1}\right)$ and $\left(A_{2}, d_{2}\right)$ are $\mathbb{A}$ chain complexes and $f: A_{1} \rightarrow A_{2}$ is an $\mathbb{A}$ chain map, we can form the mapping cone $\mathbb{M}(f)$ of $f$, whose underlying complex is the direct sum $A_{1} \oplus A_{2}$, which is equipped with the differential

$$
d_{\mathbb{M}}=\left(\begin{array}{cc}
d_{1} & 0 \\
f & -d_{2}
\end{array}\right) .
$$

The chain complex $\mathbb{M}(f)$ inherits the structure of an $\mathbb{A}$-module from $A_{1}$ and $A_{2}$, and its differential respects the $\mathbb{A}$-module structure, since $d_{1}$ and $d_{2}$ do so and $f$ is an $\mathbb{A}$ chain map. The following lemma follows immediately.

Lemma 3.7. With the above notation, we have $\mathbb{M}(f)(\mathbb{B})=\mathbb{M}\left(f^{\mathbb{B}}\right)$.

There is a short exact sequence of $\mathbb{A}$ chain complexes

$$
0 \longrightarrow A_{2}(\mathbb{B}) \stackrel{\imath^{\mathbb{B}}}{\longrightarrow} \mathbb{M}(f)(\mathbb{B}) \stackrel{\pi^{\mathbb{B}}}{\longrightarrow} A_{1}(\mathbb{B}) \longrightarrow 0
$$

induced from the natural sequence

$$
0 \longrightarrow A_{2} \stackrel{\imath}{\longrightarrow} \mathbb{M}(f) \stackrel{\pi}{\longrightarrow} A_{1} \longrightarrow 0 .
$$

For each test ring $\mathbb{B}$ for $\mathbb{A}$ we thus obtain a long exact sequence in homology

$$
\ldots \longrightarrow H\left(A_{2}, d_{2} ; \mathbb{B}\right) \longrightarrow H\left(\mathbb{M}(f), d_{\mathbb{M}} ; \mathbb{B}\right) \longrightarrow H\left(A_{1}, d_{1} ; \mathbb{B}\right) \stackrel{f_{*}^{\mathbb{B}}}{\longrightarrow} H\left(A_{2}, d_{2} ; \mathbb{B}\right) \longrightarrow \ldots
$$

The construction of the mapping cone is natural in the sense that a commutative diagram of $\mathbb{A}$ chain maps

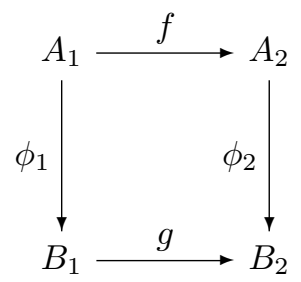


induces an $\mathbb{A}$-chain map $\mathfrak{m}\left(\phi_{1}, \phi_{2}\right): \mathbb{M}(f) \rightarrow \mathbb{M}(g)$ such that there is a homotopy commutative diagram with exact rows

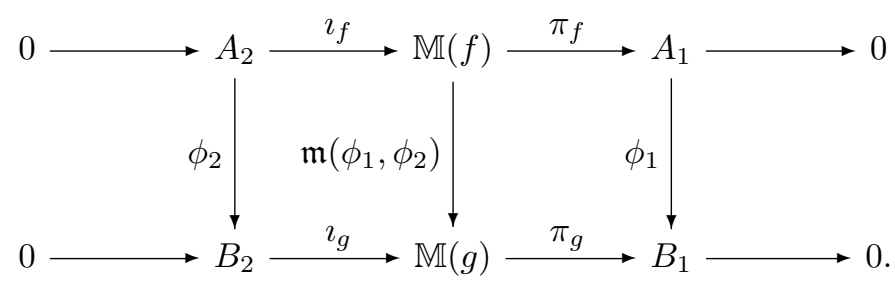

The following lemma is the main algebraic ingredient in the study of holomorphic triangles in this paper.

Lemma 3.8. (c.f. lemma 4.4 from OS6]) Let $\left\{\left(A_{i}, d_{i}\right)\right\}_{i=1}^{\infty}$ be a collection of $\mathbb{A}$ chain complexes and $\left\{f_{i}: A_{i} \rightarrow A_{i+1}\right\}$ be a collection of $\mathbb{A}$ chain maps between these complexes which satisfy the following two properties:

(1) There are $\mathbb{A}$ homomorphisms $H_{i}: A_{i} \rightarrow A_{i+2}$ such that

$$
f_{i+1} \circ f_{i}=H_{i} \circ d_{i}+d_{i+2} \circ H_{i}
$$

i.e. $f_{i+1} \circ f_{i}$ is null-homotopic via $\mathbb{A}$ chain homotopy maps $H_{i}$.

(2)The difference

$$
f_{i+2} \circ H_{i}-H_{i+1} \circ f_{i}: A_{i} \rightarrow A_{i+3}
$$

is a homotopy equivalence for $i=1,2, \ldots$.

Then $\mathbb{M}\left(f_{i}\right)$ is homotopy equivalent to $A_{i+2}$ for $i \geq 2$. Moreover, if

$$
f_{i+2} \circ H_{i}-H_{i+1} \circ f_{i}: A_{i} \rightarrow A_{i+3}
$$

is a $\mathfrak{B}$-isomorphism for some family $\mathfrak{B}$ of test rings for $\mathbb{A}$ and for $i=1,2, \ldots$, then $\mathbb{M}\left(f_{i}\right)$ is $\mathfrak{B}$-isomorphic to $A_{i+2}$ for $i \geq 2$.

Proof. The maps $\phi_{i}=(-1)^{i}\left(f_{i+2} \circ H_{i}-H_{i+1} \circ f_{i}\right): A_{i} \rightarrow A_{i+3}$ are $\mathbb{A}$ chain maps, making the following diagram homotopy commutative

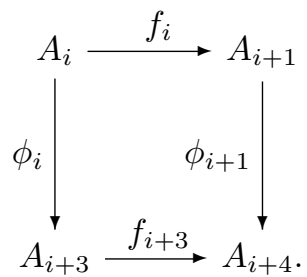

In fact, using the first property in the statement of the lemma we will have

$$
\phi_{i+1} \circ f_{i}-f_{i+3} \circ \phi_{i}=(-1)^{i}\left(\left(H_{i+2} \circ H_{i}\right) \circ d_{i}-d_{i+4} \circ\left(H_{i+2} \circ H_{i}\right)\right),
$$

and $\phi_{i+1} \circ f_{i}-f_{i+3} \circ \phi_{i}$ is thus null-homotopic. Let us denote $H_{i+2} \circ H_{i}$ by $L_{i}: A_{i} \rightarrow A_{i+4}$. We then define $\alpha_{i}: \mathbb{M}\left(f_{i}\right) \rightarrow A_{i+2}$ and $\beta_{i}: A_{i} \rightarrow \mathbb{M}\left(f_{i+1}\right)$ by

$$
\alpha_{i}\left(a_{i}, a_{i+1}\right)=f_{i+1}\left(a_{i+1}\right)-H_{i}\left(a_{i}\right), \quad \& \quad \beta_{i}\left(a_{i}\right)=\left(f_{i}\left(a_{i}\right), H_{i}\left(a_{i}\right)\right)
$$


respectively. Then $\alpha_{i+1} \circ \beta_{i}=(-1)^{i} \phi_{i}$ is a homotopy equivalence by the second property above. All the squares in the following diagram commute up to homotopy

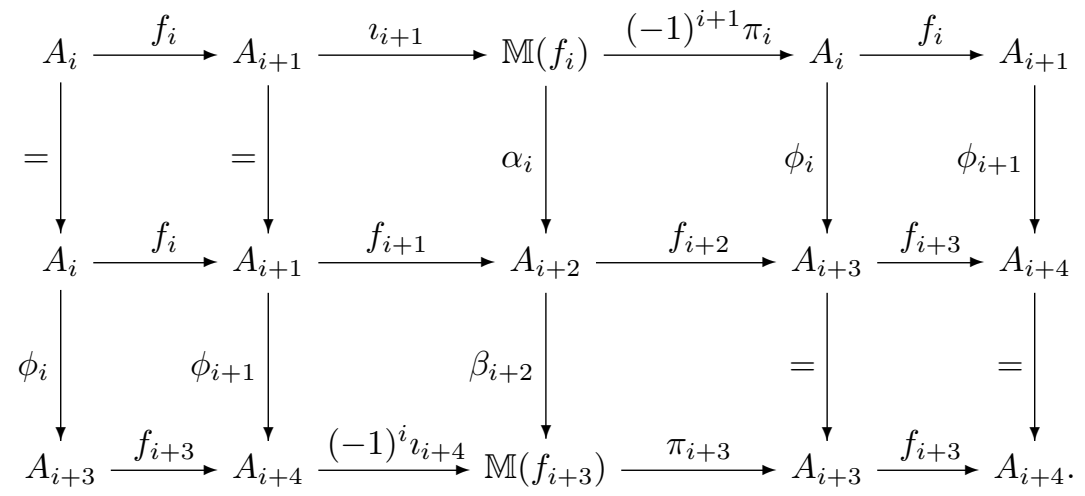

The commutativity of the two squares on the right and the two squares on the left already follows from the commutativity of the square in equation 5 . The definition of $\alpha_{i}$ and $\beta_{i+2}$ imply the equalities

$$
f_{i+2}=\pi_{i+3} \circ \beta_{i+2}, \quad \& f_{i+1}=\alpha_{i} \circ \imath_{i+1} .
$$

For the remaining two squares, let us define

$$
\begin{array}{ll}
K_{i}^{1}: \mathbb{M}\left(f_{i}\right) \rightarrow A_{i+3}, & K_{i}^{1}\left(a_{i}, a_{i+1}\right):=H_{i+1}\left(a_{i+1}\right), \\
K_{i}^{2}: A_{i} \rightarrow \mathbb{M}\left(f_{i+2}\right), & K_{i}^{2}\left(a_{i}\right)=\left(H_{i}\left(a_{i}\right), 0\right)
\end{array}
$$

We can then compute

$$
\begin{aligned}
& (-1)^{i+1} \phi_{i} \circ \pi_{i}-f_{i+2} \circ \alpha_{i}=K_{i}^{1} \circ d_{\mathbb{M}_{i}}-d_{i+3} \circ K_{i}^{1}, \quad \& \\
& \beta_{i+2} \circ f_{i+1}-(-1)^{i} u_{i+4} \circ \phi_{i+1}=K_{i+1}^{2} \circ d_{i+1}+d_{\mathbb{M}_{i+3}} \circ K_{i+1}^{2},
\end{aligned}
$$

where $d_{\mathbb{M}_{i}}$ denotes the differential of $\mathbb{M}_{i}=\mathbb{M}\left(f_{i}\right)$. We first claim that

$$
F_{i}=\beta_{i+2} \circ \alpha_{i}: \mathbb{M}\left(f_{i}\right) \rightarrow \mathbb{M}\left(f_{i+3}\right)
$$

is a chain homotopy equivalence. In fact, note that

$$
\begin{aligned}
F_{i}\left(a_{i}, a_{i+1}\right) & =\beta_{i+2}\left(f_{i+1}\left(a_{i+1}\right)-H_{i}\left(a_{i}\right)\right) \\
& =\left(f_{i+2}\left(H_{i}\left(a_{i}\right)-f_{i+1}\left(a_{i+1}\right)\right), H_{i+2}\left(H_{i}\left(a_{i}\right)-f_{i+1}\left(a_{i+1}\right)\right)\right) \\
& =\mathfrak{m}\left(\phi_{i}, \phi_{i+1}\right)\left(a_{i}, a_{i+1}\right)+\left(d_{\mathbb{M}_{i+3}} \circ H^{i}+H^{i+3} \circ d_{\mathbb{M}_{i}}\right)\left(a_{i}, a_{i+1}\right) \\
\text { where } & \left\{\begin{array}{l}
H^{i}\left(a_{i}, a_{i+1}\right):=\left(H_{i+1}\left(a_{i+1}\right), 0\right), \\
\mathfrak{m}\left(\phi_{i}, \phi_{i+1}\right)\left(a_{i}, a_{i+1}\right):=\left((-1)^{i-1} \phi_{i}\left(a_{i}\right),(-1)^{i} \phi_{i+1}\left(a_{i+1}\right)-L_{i}\left(a_{i}\right)\right)
\end{array}\right.
\end{aligned}
$$

Since $\mathfrak{m}\left(\phi_{i}, \phi_{i+1}\right)$ is a chain homotopy equivalence, it follows that the same is true for $F_{i}$. Since $\alpha_{i+3} \circ \beta_{i+2}=(-1)^{i} \phi_{i+2}$ is a chain homotopy equivalence as well, it follows that $\alpha_{2}$ is a chain homotopy equivalence (one needs to use the fact that $\alpha_{i+3}, \beta_{i+2}$ and $F_{i}$ are all chain maps).

For the $\mathfrak{B}$-isomorphism statement, note that for any test ring $\mathbb{B} \in \mathfrak{B}$ for the ring $\mathbb{A}$, we may replace the complexes $A_{i}$ with $A_{i}(\mathbb{B})$ and $\mathbb{M}\left(f_{i}\right)$ with $\mathbb{M}\left(f_{i}^{\mathbb{B}}\right)$ in the commutative diagram 6. Then the maps induced on homology associated with the first and the third row of the above diagram are exact. From the five lemma, it follows that the map induced on homology by $\beta_{i+2}^{\mathbb{B}} \circ \alpha_{i}^{\mathbb{B}}$ is an isomorphism. Since 
$\alpha_{i+3} \circ \beta_{i+2}=\phi_{i+2}$ is a $\mathfrak{B}$-isomorphism, we conclude that $\beta_{i+2}$, and hence $\alpha_{i}$ are $\mathfrak{B}$-isomorphisms as well.

3.3. Filtration by a $\mathbb{Z}$-module. Let us assume that $\mathbb{A}$ is an algebra over $\mathbb{Z}$ which is generated, as a free module over $\mathbb{Z}$, by a set $G(\mathbb{A})$ of generators. We will assume that $1 \in G(\mathbb{A})$. The choice of this basis for $\mathbb{A}$ as a free module over $\mathbb{Z}$ will be implicit in our notation. Furthermore, let $\mathbb{H}$ be a $\mathbb{Z}$-module.

Definition 3.9. By a filtration for $\mathbb{A}$ with values in $\mathbb{H}$ we mean a choice of the basis $G(\mathbb{A})$ for the free $\mathbb{Z}$-module $\mathbb{A}$, and a map

$$
\chi: G(\mathbb{A}) \longrightarrow \mathbb{H}
$$

which satisfies $\chi(1)=0$ and $\chi(a b)=\chi(a)+\chi(b)$ for all $a, b \in G(\mathbb{A})$. The pair $(\mathbb{A}, \chi: G(\mathbb{A}) \rightarrow \mathbb{H})$ is called a coefficient ring filtered by $\mathbb{H}$. We will typically drop $\chi$ and the choice of $G(\mathbb{A})$ from the notation, if there is no confusion, and will denote the filtered ring by the pair $(\mathbb{A}, \mathbb{H})$.

Suppose that $\phi_{\mathbb{B}}: \mathbb{A} \rightarrow \mathbb{B}$ is a test ring for $\mathbb{A}$ which is a free $\mathbb{Z}$-module on its own with basis $G(\mathbb{B})$, and that

is a filtration for $\mathbb{B}$.

$$
\chi_{\mathbb{B}}: G(\mathbb{B}) \longrightarrow \mathbb{H}
$$

Definition 3.10. We say that $\chi_{\mathbb{B}}$ is compatible with $\chi$ if

$$
\phi_{\mathbb{B}}(a) \in\left\langle\chi_{\mathbb{B}}^{-1}(\chi(a))\right\rangle_{\mathbb{Z}} \subset \mathbb{B}, \quad \forall a \in G(\mathbb{A}) .
$$

If this is the case, we will call the triple $\left(\mathbb{B}, \phi_{\mathbb{B}}, \chi_{\mathbb{B}}\right)$ a filtered test $\operatorname{ring}$ for $(\mathbb{A}, \mathbb{H})$. Again, when there is no confusion we will denote this triple by $(\mathbb{B}, \mathbb{H})$.

Let us assume that $(C, d)$ is an $\mathbb{A}$ chain complex, that $\chi: G(\mathbb{A}) \rightarrow \mathbb{H}$ is a filtration for $\mathbb{A}$, and that $C$ is freely generated over $\mathbb{A}$ by some subset $I$ of $C$.

Definition 3.11. We say that the $\mathbb{A}$ chain complex $(C, d)$ is a filtered $(\mathbb{A}, \mathbb{H})$ chain complex if there is a basis $I \subset C$ for $C$ over $\mathbb{A}$ and a filtration

$$
\chi: I \times I \rightarrow \mathbb{H} \text {, }
$$

which satisfies:

1) $\chi\left(c_{1}, c_{2}\right)=-\chi\left(c_{2}, c_{1}\right)$ for all $c_{1}, c_{2} \in I$.

2) $\chi\left(c_{1}, c_{2}\right)+\chi\left(c_{2}, c_{3}\right)=\chi\left(c_{1}, c_{3}\right)$, for all $c_{1}, c_{2}, c_{3} \in I$.

3) For any $c \in I, d(c)=\sum_{i=1}^{N} a_{i} c_{i}$, with $c_{1}, \ldots, c_{N} \in I$ and $a_{1}, \ldots, a_{N} \in G(\mathbb{A})$, such that

$$
\chi\left(c, c_{i}\right)=\chi\left(a_{i}\right), \quad \forall i \in\{1, \ldots, N\} .
$$

We are of course abusing the notation by denoting both the filtration of $C$ and the filtration of $\mathbb{A}$ by $\chi$.

If $(C, d)$ is a filtered $(\mathbb{A}, \mathbb{H})$ chain complex as above, one may think of $\chi\left(c_{1}, c_{2}\right)$ as the difference $\chi\left(c_{1}\right)-\chi\left(c_{2}\right)$, where $\chi: I \rightarrow \mathbb{S}$ and $\mathbb{S}$ is an affine space over the module $\mathbb{H}$. With this new notation, $\chi($ a.c $)$ for $a \in G(\mathbb{A})$ and $c \in I$ may be defined as $\chi(a)+\chi(c) \in \mathbb{S}$.

Clearly, taking the tensor product of $(C, d)$ with any filtered test ring $(\mathbb{B}, \mathbb{H})$ results in a filtered $(\mathbb{B}, \mathbb{H})$ chain complex. 
Definition 3.12. An $\mathbb{A}$ chain map $f:\left(C_{1}, d_{1}\right) \rightarrow\left(C_{2}, d_{2}\right)$ between $(\mathbb{A}, \mathbb{H})$ chain complexes $\left(C_{i}, d_{i}\right)$ with basis $I_{i}, i=1,2$ and filtrations $\chi_{1}, \chi_{2}$ is called a filtered $(\mathbb{A}, \mathbb{H})$ chain map if for all $c, c^{\prime} \in I_{1}$ we may write

$$
f(c)=\sum_{i=1}^{N} a_{i} b_{i}, \quad f\left(c^{\prime}\right)=\sum_{i=j}^{M} a_{j}^{\prime} b_{j}^{\prime}, \quad a_{i}, a_{j}^{\prime} \in G(\mathbb{A}), \& b_{i}, b_{j}^{\prime} \in I_{2},
$$

such that for any $i=1, \ldots, N$ and $j=1, \ldots, M$ we have

$$
\chi_{1}\left(c, c^{\prime}\right)=\chi_{2}\left(b_{i}, b_{j}^{\prime}\right)+\chi\left(a_{i}\right)-\chi\left(a_{j}^{\prime}\right) .
$$

In particular, if for some affine space $\mathbb{S}$ over $\mathbb{H}$, there are maps $\chi_{i}: I_{i} \rightarrow \mathbb{S}$ which satisfy $\chi_{i}\left(c_{1}, c_{2}\right)=\chi_{i}\left(c_{1}\right)-\chi_{i}\left(c_{2}\right)$ for $i=1,2$ and $c_{1}, c_{2} \in I_{i}$, the above condition may be translated to $\chi_{2}\left(a_{i} b_{i}\right)=\chi_{1}(c)$ for $i=1, \ldots, N$, whenever $f(c)=\sum_{i=1}^{N} a_{i} b_{i}$ with $a_{i} \in G(\mathbb{A})$ and $b_{i} \in I_{2}$.

Similarly, we may define the notion of a chain homotopy respecting the filtrations (i.e. $(\mathbb{A}, \mathbb{H})$ chain homotopy), and filtered $(\mathbb{A}, \mathbb{H})$ chain homotopy equivalence. Mapping cones of filtered $(\mathbb{A}, \mathbb{H})$ chain maps are filtered $(\mathbb{A}, \mathbb{H})$ chain complexes. Moreover, the following refinement of lemma 3.8 may be proved with with a similar argument.

Lemma 3.13. With the notation of lemma 3.8, if the $\mathbb{A}$ chain complexes $A_{i}$ are all filtered $(\mathbb{A}, \mathbb{H})$ chain complexes, the $\mathbb{A}$ chain maps $f_{i}$, as well as the $\mathbb{A}$ homomorphisms $H_{i}$ are all $(\mathbb{A}, \mathbb{H})$ filtered, and $f_{i+2} \circ H_{i}-H_{i+1} \circ f_{i}$ are all filtered $(\mathbb{A}, \mathbb{H})$ chain homotopy equivalences, $\mathbb{M}\left(f_{i}\right)$ is filtered $(\mathbb{A}, \mathbb{H})$ chain homotopy equivalent to $A_{i+2}$.

3.4. The algebra associated with the boundary of a sutured manifold. Let $(X, \tau)$ be a balanced sutured manifold. We will assume that

$$
\mathfrak{R}^{-}(\tau)=\bigcup_{i=1}^{k} R_{i}^{-}, \quad \& \mathfrak{R}^{+}(\tau)=\bigcup_{j=1}^{l} R_{j}^{+} .
$$

Here $R_{i}^{-}$and $R_{j}^{+}$are the connected components of $\mathfrak{R}^{-}(\tau)$ and $\mathfrak{R}^{+}(\tau)$ respectively, for $i=1, \ldots, k$ and $j=1, \ldots, l$, and let $g_{i}^{-}$denote the genus of $R_{i}^{-}$and $g_{j}^{+}$denote the genus of $R_{j}^{+}$. The set of sutures $\tau=\left\{\gamma_{1}, \ldots, \gamma_{\kappa}\right\}$ determines an algebra over $\mathbb{Z}$ as follows. Consider the free $\mathbb{Z}$-algebra

$$
\mathbb{Z}[\kappa]:=\left\langle\lambda_{1}, \ldots, \lambda_{\kappa}\right\rangle_{\mathbb{Z}}:=\mathbb{Z}\left[\lambda_{1}, \ldots, \lambda_{\kappa}\right],
$$

and consider the following elements in it

$$
\begin{aligned}
& \lambda^{-}=\lambda^{-}(\tau):=\sum_{i=1}^{k} \lambda\left(R_{i}^{-}\right), \quad \& \quad \lambda^{+}=\lambda^{+}(\tau):=\sum_{i=1}^{l} \lambda\left(R_{i}^{+}\right), \quad \text { where } \\
& \lambda_{i}^{-}=\lambda\left(R_{i}^{-}\right):=\prod_{\gamma_{j} \subset \partial R_{i}^{-}} \lambda_{j}, \quad 1 \leq i \leq k, \quad \& \\
& \lambda_{i}^{+}=\lambda\left(R_{i}^{+}\right):=\prod_{\gamma_{j} \subset \partial R_{i}^{+}} \lambda_{j}, \quad 1 \leq i \leq l .
\end{aligned}
$$


Consider the following quotients of $\left\langle\lambda_{1}, \ldots, \lambda_{\kappa}\right\rangle$, which are all finitely generated algebras over $\mathbb{Z}$ :

$$
\begin{aligned}
& \widetilde{\mathbb{A}}_{\tau}:=\frac{\left\langle\lambda_{1}, \ldots, \lambda_{\kappa}\right\rangle_{\mathbb{Z}}}{\left\langle\lambda_{i}^{-} \mid g_{i}^{-}>0\right\rangle_{\mathbb{Z}[\kappa]}+\left\langle\lambda_{j}^{+} \mid g_{j}^{+}>0\right\rangle_{\mathbb{Z}[\kappa]}}, \\
& \mathbb{A}_{\tau}:=\frac{\left\langle\lambda_{1}, \ldots, \lambda_{\kappa}\right\rangle_{\mathbb{Z}}}{\left\langle\lambda^{+}(\tau)-\lambda^{-}(\tau)\right\rangle_{\mathbb{Z}[\kappa]}+\left\langle\lambda_{i}^{-} \mid g_{i}^{-}>0\right\rangle_{\mathbb{Z}[\kappa]}+\left\langle\lambda_{j}^{+} \mid g_{j}^{+}>0\right\rangle_{\mathbb{Z}[\kappa]}}, \quad \& \\
& \widehat{\mathbb{A}}_{\tau}:=\frac{\left\langle\lambda_{1}, \ldots, \lambda_{\kappa}\right\rangle_{\mathbb{Z}}}{\left\langle\lambda_{i}^{-} \mid i=1, \ldots, k\right\rangle_{\mathbb{Z}[\kappa]}+\left\langle\lambda_{j}^{+} \mid j=1, \ldots, l\right\rangle_{\mathbb{Z}[\kappa]}} .
\end{aligned}
$$

Clearly $\mathbb{A}=\mathbb{A}_{\tau}, \widehat{\mathbb{A}}=\widehat{\mathbb{A}}_{\tau}$ and $\widetilde{\mathbb{A}}=\widetilde{\mathbb{A}}_{\tau}$ are all generated, as modules over $\mathbb{Z}$, by elements of the form $\prod_{i=1}^{\kappa} \lambda_{i}^{a_{i}}$, where $a_{i}$ are non-negative integers. We will denote the set of all such monomials by $G(\mathbb{A})=G(\widetilde{\mathbb{A}})$. The ring $\mathbb{A}_{\tau}$ will be used as the coefficient ring for the Ozsváth-Szabó chain complexes which we introduce in this paper, while $\widetilde{\mathbb{A}}_{\tau}$ is used to make the admissibility criteria for the Heegaard diagrams slightly stronger, and is not really essential in our construction. The ring $\widehat{\mathbb{A}}_{\tau}$ will appear when we discuss a generalization of Juhász' surface decomposition formula from [Ju1] later in [?].

One may define a natural map, called the Poincaré duality character in this paper, from $G(\mathbb{A})$ to the $\mathbb{Z}$-module $\mathbb{H}=\mathbb{H}_{\tau}:=\mathrm{H}^{2}(X, \partial X, \mathbb{Z})$ by

$$
\begin{aligned}
& \chi: G(\mathbb{A}) \longrightarrow \mathbb{H}=\mathrm{H}^{2}(X, \partial X ; \mathbb{Z}), \\
& \chi\left(\prod_{i=0}^{\kappa} \lambda_{i}^{a_{i}}\right):=a_{1} \operatorname{PD}\left[\gamma_{1}\right]+\ldots+a_{\kappa} \operatorname{PD}\left[\gamma_{\kappa}\right], \quad \forall a_{1}, . ., a_{\kappa} \in \mathbb{Z}^{\geq 0} .
\end{aligned}
$$

As defined, $\chi$ is just a map from the set of generators for $\mathbb{Z}[\kappa]$ to $\mathrm{H}^{2}(X, \partial X, \mathbb{Z})$. However, since $\chi\left(\lambda\left(R_{i}^{-}\right)\right)=-\mathrm{PD}\left[\partial R_{i}^{-}\right]=0$ and $\chi\left(\lambda\left(R_{j}^{+}\right)\right)=\mathrm{PD}\left[\partial R_{j}^{+}\right]=0$ for all $i=1, \ldots, k$ and $j=1, \ldots, l$, the map is well-defined on $G(\mathbb{A})$ and $G(\widetilde{\mathbb{A}})$. The Poincaré duality character gives filtrations of $\mathbb{A}$ and $\widetilde{\mathbb{A}}$ by $\mathbb{H}=\mathrm{H}^{2}(X, \partial X ; \mathbb{Z})$.

We may also define a map from the set of positive Whitney disks associated with a Heegaard diagram $(\Sigma, \boldsymbol{\alpha}, \boldsymbol{\beta}, \mathbf{z})$ for $(X, \tau)$ to $G(\mathbb{A})$ and $G(\widetilde{\mathbb{A}})$ by computing the local multiplicities of the domain associated with each disk at the marked points in $\mathbf{z}$ :

$$
\begin{aligned}
& \lambda=\lambda_{\mathbf{z}}: \coprod_{\mathbf{x}, \mathbf{y} \in \mathbb{T}_{\alpha} \cap \mathbb{T}_{\beta}} \pi_{2}^{+}(\mathbf{x}, \mathbf{y}) \longrightarrow G(\mathbb{A}) \\
& \lambda(\phi):=\prod_{i=1}^{\kappa} \lambda_{i}^{n_{z_{i}}(\phi)}, \quad \forall \mathbf{x}, \mathbf{y} \in \mathbb{T}_{\alpha} \cap \mathbb{T}_{\beta}, \& \forall \phi \in \pi_{2}^{+}(\mathbf{x}, \mathbf{y}) .
\end{aligned}
$$

Similarly, we may define $\tilde{\lambda}=\widetilde{\lambda}_{\mathbf{z}}: \pi_{2}^{+}(\mathbf{x}, \mathbf{y}) \rightarrow G(\widetilde{\mathbb{A}})$. Note theta $\lambda$ is just the composition of $\widetilde{\lambda}$ with the quotient homomorphism $\widetilde{\mathbb{A}} \rightarrow \mathbb{A}$. The composition $\chi(\lambda(\phi))$ in $\mathrm{H}^{2}(X, \partial X, \mathbb{Z})$ will be denoted by $\widehat{H}(\phi)$ for any $\phi \in \pi_{2}^{+}(\mathbf{x}, \mathbf{y})$. Of course, 
the definition of $\widehat{H}(\phi)$ may be extended to arbitrary $\phi \in \pi_{2}(\mathbf{x}, \mathbf{y})$ by setting

$$
\widehat{H}(\phi)=\sum_{i=1}^{\kappa} n_{z_{i}}(\phi) \operatorname{PD}\left[\gamma_{i}\right] .
$$

Thus, corollary 2.9 may be re-stated as

$$
\phi \in \pi_{2}(\mathbf{x}, \mathbf{y}) \Rightarrow \underline{\mathfrak{s}}(\mathbf{x})-\underline{\mathfrak{s}}(\mathbf{y})=\widehat{H}(\phi) .
$$

Lemma 3.14. With the above notation, if $\lambda \in G\left(\widetilde{\mathbb{A}}_{\tau}\right)$ is a monomial and $\lambda^{n}=0$ for some positive integer $n \in \mathbb{Z}^{+}$, then $\lambda=0$ in $\widetilde{\mathbb{A}}_{\tau}$. In other words, $\widetilde{\mathbb{A}}_{\tau}$ does not contain any non-trivial nilpotent monomials.

Proof. It suffices to show that $\lambda^{2}=0$ implies $\lambda=0$. Since the monomials are linearly independent (over $\mathbb{Q}$ ) in $\mathbb{Z}[\kappa], \lambda^{2}=0$ implies that at least one monomial in

$$
\left\{\lambda_{i}^{+} \mid g_{i}^{+}>0\right\} \bigcup\left\{\lambda_{i}^{-} \mid g_{i}^{-}>0\right\}
$$

divides $\lambda^{2}$ in $\mathbb{Z}[\kappa]$. Note that all the monomials in this set are square free. Thus, if $\lambda_{i}^{+}$for some index $i$ with $g_{i}^{+}>0$ divides $\lambda^{2}$ in $\mathbb{Z}[\kappa]$, it should divide $\lambda$ as well, and $\lambda=0$ in $\widetilde{\mathbb{A}}$. Similarly, if $\lambda_{i}^{-}$for some index $i$ with $g_{i}^{-}>0$ divides $\lambda^{2}$ in $\mathbb{Z}[\kappa]$, then $\lambda=0$ in $\widetilde{\mathbb{A}}$ and we are done.

Example 3.15. If the boundary of the sutured manifold $(X, \tau)$ consists of a torus $T$, and if on $T$ we have $2 n$ parallel simple closed curves $\mu_{1}, \ldots, \mu_{2 n}$, we may assume that the corresponding sutured manifold is defined by the following data:

$$
\begin{aligned}
& \tau=\left\{\mu_{1}, \ldots, \mu_{2 n}\right\}, \quad \mu_{2 n+1}:=\mu_{1}, \\
& \mathfrak{R}^{+}(\tau)=\bigcup_{j=1}^{n} R_{j}^{+}, \quad \& \quad \mathfrak{R}^{-}(\tau)=\bigcup_{j=1}^{n} R_{j}^{-} \\
& \partial R_{j}^{+}=\mu_{2 j-1}+\mu_{2 j}, \quad \& \partial R_{j}^{-}=-\mu_{2 j}-\mu_{2 j+1}, \quad j=1, \ldots, n .
\end{aligned}
$$

The sutured manifold $(X, \tau)$ determines a knot $K$ inside the closed three-manifold $Y$, obtained by gluing a 2-handle to one of $\mu_{i}$ and then a 3-handle to the spherical boundary of the new three-manifold. The torus $T$ may then be pictured as the boundary of a neighborhood of $K$ in the resulting closed three-manifold.

Let $\lambda_{j}$ denote the variable associated with the suture $\mu_{j}, j=1, \ldots, 2 n$. Then, since all the components in $\mathfrak{R}(\tau)$ are surfaces of genus zero, the following relation is the only relation satisfied in $\mathbb{A}_{\tau}$ :

$$
\lambda_{1} \lambda_{2}-\lambda_{2} \lambda_{3}+\ldots+\lambda_{2 n-1} \lambda_{2 n}-\lambda_{2 n} \lambda_{1}=0 .
$$

In other words, we have

$$
\mathbb{A}_{\tau}=\frac{\mathbb{Z}\left[\lambda_{1}, \ldots, \lambda_{2 n}\right]}{\left\langle\lambda_{1} \lambda_{2}+\ldots+\lambda_{2 n-1} \lambda_{2 n}=\lambda_{2} \lambda_{3}+\ldots+\lambda_{2 n} \lambda_{1}\right\rangle}
$$

In particular, for $n=1$, the above relation is trivial and $\mathbb{A}_{\tau}=\mathbb{Z}\left[\lambda_{1}, \lambda_{2}\right]$ is the coefficient ring used by Ozsváth and Szabó in defining $\mathrm{CF}^{-}(Y, K)$.

Suppose that $(X, \tau)$ is a balanced sutured manifold, and $H=(\Sigma, \boldsymbol{\alpha}, \boldsymbol{\beta}, \mathbf{z}=$ $\left.\left\{z_{1}, \ldots, z_{\kappa}\right\}\right)$ is a Heegaard diagram associated with it, and $\mathbb{A}_{\tau}$ be the corresponding algebra. Associated with the Heegaard diagram is a free $\mathbb{A}_{\tau}$-module generated 
by the intersection points $\mathbf{x} \in \mathbb{T}_{\alpha} \cap \mathbb{T}_{\beta}$. We will denote this free $\mathbb{A}_{\tau}$-module by $\mathrm{CF}(X, \tau ; H)$. We thus have

$$
\begin{aligned}
\mathrm{CF}(X, \tau ; H): & =\left\langle\mathbf{x} \mid \mathbf{x} \in \mathbb{T}_{\alpha} \cap \mathbb{T}_{\beta}\right\rangle_{\mathbb{A}_{\tau}} \\
& =\left\langle a \cdot \mathbf{x} \mid \mathbf{x} \in \mathbb{T}_{\alpha} \cap \mathbb{T}_{\beta}, \& a \in G\left(\mathbb{A}_{\tau}\right)\right\rangle_{\mathbb{Z}} .
\end{aligned}
$$

The assignment of relative $\operatorname{Spin}^{c}$ structures in $\mathbb{S}=\mathbb{S}_{\tau}=\operatorname{Spin}^{c}(X, \tau)$ to the intersection points in $\mathbb{T}_{\alpha} \cap \mathbb{T}_{\beta}$ by the map $\underline{\mathfrak{s}}=\mathfrak{\mathfrak { s }}_{\mathbf{z}}$ may thus be regarded as a filtration on the module $\mathrm{CF}(X, \tau ; H)$. 


\section{Admissible HeegaArd diagrams}

4.1. The notion of $\mathfrak{s}$-admissibility. Let $\left(\Sigma, \boldsymbol{\alpha}, \boldsymbol{\beta}, \mathbf{z}=\left\{z_{1}, \ldots, z_{\kappa}\right\}\right)$ be a Heegaard diagram for the balanced sutured manifold $\left(X, \tau=\left\{\gamma_{1}, \ldots, \gamma_{\kappa}\right\}\right)$. As before, we let

$$
\Sigma-\boldsymbol{\alpha}=\coprod_{i=1}^{k} A_{i}, \quad \& \quad \Sigma-\boldsymbol{\beta}=\coprod_{i=1}^{l} B_{i} .
$$

Definition 4.1. Let $\bar{X}=X(1, \ldots, \kappa)$ be the three manifold obtained by filling the sutures in $\tau$. For $\mathfrak{s} \in \operatorname{Spin}^{c}(\bar{X})$, a Heegaard diagram $(\Sigma, \boldsymbol{\alpha}, \boldsymbol{\beta}, \mathbf{z})$ is called $\mathfrak{s}$-admissible if for any nontrivial periodic domain $\mathcal{P}$ with the property $\left\langle c_{1}(\mathfrak{s}), H(\mathcal{P})\right\rangle=0$ one of the following happens,

(a) There is a point $w \in \Sigma$ such that $n_{w}(\mathcal{P})<0$.

(b) We have $\mathcal{P} \geq 0$ and $\widetilde{\lambda}(\mathcal{P})=0$ in $\widetilde{\mathbb{A}}$.

Lemma 4.2. For $\mathfrak{s} \in \operatorname{Spin}^{c}(\bar{X})$, let $(\Sigma, \boldsymbol{\alpha}, \boldsymbol{\beta}, \mathbf{z})$ be an $\mathfrak{s}$-admissible Heegaard diagram for the balanced sutured manifold $(X, \tau)$. Then for any two intersection points $\mathbf{x}, \mathbf{y} \in \mathbb{T}_{\alpha} \cap \mathbb{T}_{\beta}$ with $\mathfrak{\underline { s }}(\mathbf{x}), \underline{\mathfrak{s}}(\mathbf{y}) \in \mathfrak{s} \subset \operatorname{Spin}^{c}(X, \tau)$, and for any integer $j$ there are only finitely many $\phi \in \pi_{2}(\mathbf{x}, \mathbf{y})$ such that $\mu(\phi)=j, \mathcal{D}(\phi) \geq 0$ and $\widetilde{\lambda}(\phi) \neq 0$.

Proof. Suppose that, for an integer $j$, there are infinitely many $\phi \in \pi_{2}(\mathbf{x}, \mathbf{y})$ such that $\mu(\phi)=j, \mathcal{D}(\phi) \geq 0$ and $\widetilde{\lambda}(\phi) \neq 0$. Fix an element $\phi_{0} \in \pi_{2}(\mathbf{x}, \mathbf{y})$ with these properties. Any $\phi \in \pi_{2}(\mathbf{x}, \mathbf{y})$ with these properties can then be written as $\phi=\phi_{0}+\mathcal{P}$ where $\mathcal{P} \in \pi_{2}(\mathbf{x}, \mathbf{x})$ and $\mu(\mathcal{P})=\left\langle c_{1}(\mathfrak{s}), H(\mathcal{P})\right\rangle=0$. Thus the set

$$
Q=\left\{\mathcal{P} \in \pi_{2}(\mathbf{x}, \mathbf{x}) \mid \mu(\mathcal{P})=0, \mathcal{P}+\mathcal{D}\left(\phi_{0}\right) \geq 0, \tilde{\lambda}\left(\phi_{0}+\mathcal{P}\right) \neq 0\right\}
$$

is not finite. Let $m$ denote the total number of domains in $\Sigma-\boldsymbol{\alpha}-\boldsymbol{\beta}$, and $D_{i}$, for $i=1, \ldots, m$, denote the corresponding domains. Consider $Q$ as a subset of the set of all lattice points in the vector space

$$
V=\left\langle\mathcal{P} \in \pi_{2}(\mathbf{x}, \mathbf{x}) \mid\left\langle c_{1}(\mathfrak{s}), H(\mathcal{P})\right\rangle=0\right\rangle_{\mathbb{R}} \subset \mathbb{R}^{m} .
$$

Here, $\mathbb{R}^{m}$ is the vector space generated by the domains $D_{i}, i=1, \ldots, m$ over $\mathbb{R}$. If $Q$ is not finite, there is a sequence $\left(\mathcal{P}_{i}\right)_{i=1}^{\infty}$ in $Q$, such that $\left\|\mathcal{P}_{i}\right\| \rightarrow \infty$. By passing to a subsequence if necessary, we may assume that the sequence $\left\{\frac{\mathcal{P}_{i}}{\left\|\mathcal{P}_{i}\right\|}\right\}_{i=1}^{\infty}$ is convergent on the unit ball inside $V$. Since $\left\|\mathcal{P}_{i}\right\| \rightarrow \infty$, and $\mathcal{P}_{i}+\mathcal{D}\left(\phi_{0}\right) \geq 0$, it converges to a real vector in $\mathbb{R}^{m}$ with non-negative entries. Denote the limit of $\left(\frac{\mathcal{P}_{i}}{\left\|\mathcal{P}_{i}\right\|}\right)$ by $\widetilde{\mathcal{P}}$, which is a periodic domain with non-negative real entries. There is a positive rational periodic domain $\mathcal{P}$, sufficiently closed to $\widetilde{\mathcal{P}}$, such that

- The Maslov index $\mu(\mathcal{P})=0$, i.e. $\mathcal{P} \in V$

- If the coefficients of $\widetilde{\mathcal{P}}$ in some domain $D_{i}$ is zero, the coefficient of $\mathcal{P}$ in $D_{i}$ is zero as well.

Thus for a sufficiently large number $M, M \widetilde{\mathcal{P}}-\mathcal{P}$ is a positive periodic domain. After multiplying $\mathcal{P}$ with an appropriate positive integer $N$, we obtain a positive periodic domain $N \mathcal{P}$ with integral coefficients, and with Maslov index zero i.e. $\left\langle c_{1}(\mathfrak{s}), H(N \mathcal{P})\right\rangle=0$. The $\mathfrak{s}$-admissibility condition implies that $\widetilde{\lambda}(N \mathcal{P})=0$. Since

$$
\lim _{i \rightarrow \infty} \frac{M N}{\left\|\mathcal{P}_{i}\right\|}\left(\mathcal{D}\left(\phi_{0}\right)+\mathcal{P}_{i}\right)=M N \widetilde{\mathcal{P}}
$$


and $\mathcal{D}\left(\phi_{0}\right)+\mathcal{P}_{i} \geq 0$, there exists a sufficiently large integer $K>0$ such that

$$
\frac{M N}{\left\|\mathcal{P}_{i}\right\|}\left(\mathcal{D}\left(\phi_{0}\right)+\mathcal{P}_{i}\right)-N \mathcal{P} \geq 0, \quad \forall i>K .
$$

Note that $\left\|\mathcal{P}_{i}\right\| \rightarrow \infty$, thus for an $K$ sufficiently large we have

$$
\frac{M}{\left\|\mathcal{P}_{i}\right\|}<1, \quad \& N\left(\mathcal{D}\left(\phi_{0}\right)+\mathcal{P}_{i}\right)-N \mathcal{P} \geq 0, \quad \forall i>K .
$$

The equality $\widetilde{\lambda}(N \mathcal{P})=0$ implies that $\widetilde{\lambda}\left(\phi_{i}\right)^{N}=\widetilde{\lambda}\left(N\left(\mathcal{D}\left(\phi_{0}\right)+\mathcal{P}_{i}\right)\right)=0$ for any $i>K$. Since there are no nilpotent monomials in $\widetilde{\mathbb{A}}_{\tau}$ by lemma 3.14 , we should have $\widetilde{\lambda}\left(\phi_{i}\right)=0$ for any $i>K$, which is in contradiction with the assumption that the map $\widetilde{\lambda}$ is nonzero over the classes $\phi_{i}$.

Remark 4.3. When we use a test ring $\mathbb{B}$ for $\mathbb{A}_{\tau}$ (which comes together with a ring homomorphism $\rho_{\mathbb{B}}: \mathbb{A}_{\tau} \rightarrow \mathbb{B}$ ) as the ring of coefficients for the chain complex, it suffices to assume that the Heegaard diagram $(\Sigma, \boldsymbol{\alpha}, \boldsymbol{\beta}, \mathbf{z})$ is admissible in the following weaker sense: If $\mathcal{P}$ is a periodic domain with $\mathcal{P} \geq 0$ and $\left\langle c_{1}(\mathfrak{s}), H(\mathcal{P})\right\rangle=0$, then $\rho_{\mathbb{B}}(\lambda(\mathcal{P}))=0$. In particular, for $\mathbb{B}=\mathbb{Z}$ this gives us the notion of weak admissibility used by Juhász [Ju1]. More generally, define

$$
\mathbb{B}_{\tau}=\frac{\left\langle\lambda_{1}, \ldots, \lambda_{\kappa}\right\rangle_{\mathbb{Z}}}{\left.\left\langle\prod_{i=1}^{\kappa} \lambda_{i}^{n_{i}} \neq 1\right| n_{i} \in \mathbb{Z} \geq 0 \& \sum_{i=1}^{\kappa} n_{i}\left[\gamma_{i}\right]=0 \text { in } \mathrm{H}_{1}(X ; \mathbb{Z}) / \text { Tors }\right\rangle} .
$$

Clearly $\mathbb{B}_{\tau}$ is a quotient of $\mathbb{A}_{\tau}$. Let us denote the quotient map by

$$
\rho_{\tau}: \mathbb{A}_{\tau} \longrightarrow \mathbb{B}_{\tau} .
$$

Any positive periodic domain $\mathcal{P}$ with $\lambda_{\mathbf{z}}(\mathcal{P})=\prod_{i=1}^{\kappa} \lambda_{i}^{n_{i}}$ determines a 2-chain in $X$ with boundary equal to $\sum_{i=1}^{\kappa} n_{i} \gamma_{i}$. This implies that $\rho_{\tau}\left(\lambda_{\mathbf{z}}(\mathcal{P})\right)=0$, unless $n_{1}=n_{2}=\ldots=n_{\kappa}=0$. Thus, the notion of admissibility for the coefficient ring $\mathbb{B}_{\tau}$ is a direct consequence of weak admissibility in the sense of Juhász [Ju1].

4.2. Existence of $\mathfrak{s - a d m i s s i b l e ~ H e e g a a r d ~ d i a g r a m s . ~ . ~ P e r f o r m i n g ~ s p e c i a l ~ i s o - ~}$ topies on the curves in $\boldsymbol{\alpha}$, as in [OS5], produces $\mathfrak{s}$-admissible Heegaard diagrams.

Definition 4.4. Let $\gamma$ be an oriented simple closed curve in $\Sigma$. Consider the coordinate system $(t, \theta) \in(-\epsilon, \epsilon) \times S^{1}$ in a neighborhood of $\gamma=\{0\} \times S^{1}$. The diffeomorphism of $\Sigma$ obtained by integrating a vector field $\zeta$ supported in this neighborhood of $\gamma$ with the property $d \theta(\zeta)>0$ is called winding along $\gamma$. Let $\alpha$ be a simple closed curve which intersects $\gamma$ in one point and $\phi$ be a winding around $\gamma$. If $\phi(\alpha)$ intersects $\alpha$ in $2 n$ points then we say that $\phi$ is an isotopy which winds $\alpha$ $n$-times around $\gamma$.

Lemma 4.5. Let $(X, \tau)$ be a balanced sutured manifold as before, $\bar{X}$ be the threemanifold obtained from $(X, \tau)$ by filling the sutures, and $\mathfrak{s} \in \operatorname{Spin}^{c}(\bar{X})$ be a $\operatorname{Spin}^{c}$ structure. Then $(X, \tau)$ admits an $\mathfrak{s}$-admissible Heegaard diagram. Moreover, every Heegaard diagram $(\Sigma, \boldsymbol{\alpha}, \boldsymbol{\beta}, \mathbf{z})$ for $(X, \tau)$ may be modified to an $\mathfrak{s - a d m i s s i b l e ~ H e e - ~}$ gaard diagram by performing isotopies (supported away from the marked points) on the curves in $\boldsymbol{\alpha}$. 
Proof. Let $(\Sigma, \boldsymbol{\alpha}, \boldsymbol{\beta}, \mathbf{z})$ be a Heegaard diagram for $(X, \tau)$. Let

$$
\Sigma-\boldsymbol{\alpha}=\coprod_{i=1}^{k} A_{i}, \quad \& \quad \Sigma-\boldsymbol{\beta}=\coprod_{i=1}^{l} B_{i},
$$

be the connected components in the complement of $\boldsymbol{\alpha}$ and $\boldsymbol{\beta}$ respectively. For any $i \in\{1, \ldots, k\}$ and $j \in\{1, \ldots, l\}$ we can find a curve $\gamma$ such that $\gamma$ connects $\partial A_{i}$ to $\partial B_{j}$, and avoids $A_{i}$ and $B_{j}$. By finger moving those $\alpha$ curves which intersect the curve $\gamma$ (simultaneously) along $\gamma$, we create a new Heegaard diagram with the property that $A_{i} \cap B_{j} \neq \emptyset$. Repeating this procedure for all $i=1, \ldots, k$ and $j=1, \ldots, l$, we may thus assume that

$$
A_{i} \cap B_{j} \neq \emptyset, \quad \forall i=1, \ldots, k, j=1, \ldots, l .
$$

Let $\boldsymbol{\alpha}_{0}$ be a set of disjoint simple closed curves on $\Sigma$, disjoint from $\boldsymbol{\alpha}$, such that $\Sigma-\boldsymbol{\alpha}-\boldsymbol{\alpha}_{0}$ has the same number of connected components as $\Sigma-\boldsymbol{\alpha}$, and all of its connected components have genus zero. Furthermore, let $\boldsymbol{\alpha}=\boldsymbol{\alpha}_{1} \cup \boldsymbol{\alpha}_{2}$ where

$$
\boldsymbol{\alpha}_{2}=\left\{\alpha_{i} \in \boldsymbol{\alpha} \mid \exists j \quad \alpha_{i} \subset \partial A_{j}\right\} .
$$

For $i=0,1,2$, let us denote the number of elements in $\boldsymbol{\alpha}_{i}$ by $\ell_{i}$. Thus, in particular, $\ell=\ell_{1}+\ell_{2}$.

We define a graph $G$ with $k$ vertices corresponding to the domains $A_{1}, \ldots, A_{k}$. The edges of $G$ correspond to the elements of $\boldsymbol{\alpha}_{2}$, i.e. if $\alpha \in \boldsymbol{\alpha}_{2}$ is a curve in $\partial A_{i} \cap \partial A_{j}$, we put an edge in $G$ connecting $A_{i}$ to $A_{j}$ associated with $\alpha$. If $=\Sigma\left[\boldsymbol{\alpha}_{1}\right]$ is the surface obtained from $\Sigma$ by surgering out the elements of $\boldsymbol{\alpha}_{1}$, each loop in $G$ corresponds to a homologically nontrivial simple closed curve in $\Sigma\left[\boldsymbol{\alpha}_{1}\right]$ which is disjoint from $\boldsymbol{\alpha}_{0}$. In other words, each loop in $G$ corresponds to a homologically nontrivial simple closed curve in $\Sigma\left[\boldsymbol{\alpha}_{0} \cup \boldsymbol{\alpha}_{1}\right]$. Furthermore, $h=\operatorname{dim}\left(\mathrm{H}_{1}(G, \mathbb{Z})\right)$ is the genus of $\Sigma\left[\boldsymbol{\alpha}_{0} \cup \boldsymbol{\alpha}_{1}\right]$. One may easily compute $h=\ell_{2}-k+1$.

Consider a set of pairwise disjoint simple closed curves

$$
\gamma=\gamma_{1} \cup \gamma_{2}=\left\{\gamma^{1}, \ldots, \gamma^{\ell_{1}}\right\} \bigcup\left\{\gamma^{\ell_{1}+1}, \ldots, \gamma^{\ell-k+1}\right\}
$$

on $\Sigma$ with the following properties. First of all, we assume that $\gamma_{1}$ is a dual set for $\boldsymbol{\alpha}_{1}$ i.e. each element of $\boldsymbol{\gamma}_{1}$ intersects exactly one element of $\boldsymbol{\alpha}_{1}$ with intersection number one, and for each element of $\boldsymbol{\alpha}_{1}$ there is one element of $\gamma_{1}$ intersecting it (with intersection number one). Furthermore, we assume that

$$
\gamma_{1} \cap \boldsymbol{\alpha}_{0}=\gamma_{1} \cap \boldsymbol{\alpha}_{2}=\emptyset
$$

The set $\gamma_{2}$ corresponds to a basis for $\mathrm{H}_{1}(G, \mathbb{Z})$ which is a set of disjoint, oriented, and linearly independent simple closed curves on $\Sigma\left[\boldsymbol{\alpha}_{0} \cup \boldsymbol{\alpha}_{1}\right]$. There is a one to one map $i: \gamma_{2} \rightarrow \boldsymbol{\alpha}_{2}$ with the property that for each $\gamma \in \gamma_{2}$ the curve $i(\gamma)$ has nonempty intersection with $\gamma$. In fact if this is not true, by Hall's theorem there is a subset of $\gamma_{2}$ with $n$ elements, say $\left\{\gamma^{i_{1}}, \ldots, \gamma^{i_{n}}\right\} \subset \gamma_{2}$, such that for

$$
A=\left\{\alpha \in \boldsymbol{\alpha}_{2} \mid \exists j \in\{1, \ldots, n\} \text { s.t. } \alpha \cap \gamma^{i_{j}} \neq \emptyset\right\}
$$

we have $|A|<n$. Since the sum of the genera of the connected components of $\Sigma[\boldsymbol{\alpha}]$ is $\ell_{0}, \Sigma[\boldsymbol{\alpha}-A]$ is a surface whose genus is less than or equal to $|A|+\ell_{0}$. 
Furthermore, the curves in $\left\{\gamma^{i_{1}}, \ldots, \gamma^{i_{n}}\right\} \cup \boldsymbol{\alpha}_{0}$ are linearly independent in

$$
\mathrm{H}_{1}(\Sigma[\boldsymbol{\alpha}-A], \mathbb{Z}) .
$$

Thus the genus of $\Sigma\left[\boldsymbol{\alpha}_{2}-A\right]$ is at least $n+\ell_{0}$ which is in contradiction with the assumption $|A|<n$.

Choose a parallel copy of each curve $\gamma^{i}$ for $i=1, \ldots, \ell-k+1$, with the opposite orientation and denote it by $\bar{\gamma}^{i}$. We will assume that $\bar{\gamma}^{i}$ is drawn on $\Sigma$ very close to $\gamma^{i}$. Let $v_{i} \in \gamma^{i}$ be points which are not contained in any of the $\boldsymbol{\alpha}$ or $\boldsymbol{\beta}$ curves for any $1 \leq i \leq \ell+k-1$ and denote the corresponding points on $\bar{\gamma}^{i}$ by $\bar{v}_{i}$. For any integer $N$, by winding the $\alpha$ curves $N$ times along the $\gamma$ curves we mean winding all the $\alpha$-curves which cut $\gamma^{i}$ (and hence $\bar{\gamma}^{i}$ ) $N$ times along $\gamma^{i}$ and $N$ times along $\bar{\gamma}^{i}$, for any of the curves $\gamma^{i}, i=1, \ldots, \ell-k+1$. The windings around either of $\gamma^{i}$ and $\bar{\gamma}^{i}$ will be done simultaneously for all the $\alpha$ curves, so that the new $\alpha$-curves remain disjoint from each other.

Let $Q$ be the $\mathbb{Q}$-vector space generated by the periodic domains $\mathcal{P}$ such that

$$
\mu(\mathcal{P})=\left\langle c_{1}(\mathfrak{s}), H(\mathcal{P})\right\rangle=0 .
$$

One may write $Q$ as a direct sum

$$
Q=\left(Q \cap\left\langle A_{1}, \ldots, A_{k}, B_{1}, \ldots, B_{l}\right\rangle_{\mathbb{Q}}\right) \oplus P,
$$

for sum subspace $P$ of $Q$ which is generated by the periodic domains $\left\{\mathcal{P}_{1}, \ldots, \mathcal{P}_{b}\right\}$. Thus any periodic domain $\mathcal{P}$ in the vector space $Q$ is of the form

$$
\mathcal{P}=\sum_{i=1}^{b} q_{i} \mathcal{P}_{i}+\sum_{i=1}^{k} a_{i} A_{i}+\sum_{i=1}^{l} b_{i} B_{i}
$$

where of course the coefficients $a_{i}$ and $b_{j}$ for $i=1, \ldots, k$ and $j=1, \ldots, l$ should satisfy the relation

$$
\mu\left(\sum_{i=1}^{k} a_{i} A_{i}+\sum_{i=1}^{l} b_{i} B_{i}\right)=\sum_{i=1}^{k} a_{i}\left(2-2 g\left(A_{i}\right)\right)+\sum_{i=1}^{l} b_{i}\left(2-2 g\left(B_{i}\right)\right)=0 .
$$

In the above expression $g\left(A_{i}\right)$ and $g\left(B_{j}\right)$ denote the genus of $A_{i}$ and $B_{j}$ respectively, for $i=1, \ldots, k$ and $j=1, \ldots, l$.

Corresponding to any curve $\gamma^{i} \in \boldsymbol{\gamma}$ we define a map $p_{\gamma^{i}}$ from $P$ to $\mathbb{Q}$. If $\mathcal{P} \in P$ is a periodic domain and

$$
\partial \mathcal{P}=\sum_{i=1}^{\ell} p_{i} \alpha_{i}+\sum_{i=1}^{\ell} q_{i} \beta_{i}=\partial_{\alpha} \mathcal{P}+\partial_{\beta} \mathcal{P},
$$

we may define the functions $p_{\gamma^{i}}$ by

$$
p_{\gamma^{i}}(\mathcal{P})=\sum_{j=1}^{\ell} p_{j} \cdot \#\left(\gamma^{i} \cdot \alpha_{j}\right), \quad \forall i \in\{1, \ldots, \ell-k+1\} .
$$


Here \# $\left(\gamma^{i} \cdot \alpha_{j}\right)$ denotes the intersection number of $\gamma^{i}$ with $\alpha_{j}$. If for some periodic domain $\mathcal{P} \in P$ we have $p_{\gamma^{i}}(\mathcal{P})=0$, for $1 \leq i \leq \ell-k+1$, we may conclude that

$$
\begin{aligned}
& \#\left(\partial_{\alpha} \mathcal{P} \cdot \gamma^{i}\right)=p_{\gamma^{i}}(\mathcal{P})=0, \quad \forall 1 \leq i \leq \ell-k+1 \\
\Rightarrow & \partial_{\alpha} \mathcal{P}=\partial\left(\sum_{i=1}^{k} a_{i} A_{i}\right), \quad \text { for some } a_{1}, \ldots, a_{k} \in \mathbb{Q} \\
\Rightarrow & \partial\left(\mathcal{P}-\sum_{i=1}^{k} a_{i} A_{i}\right) \in\left\langle\beta_{1}, \ldots, \beta_{\ell}\right\rangle_{\mathbb{Q}} \\
\Rightarrow & \mathcal{P}=\sum_{i=1}^{k} a_{i} A_{i}+\sum_{i=1}^{l} b_{i} B_{i}, \quad \text { for some } b_{1}, \ldots, b_{l} \in \mathbb{Q}
\end{aligned}
$$

From the assumption $\mathcal{P} \in P$ we have $\mathcal{P}=0$. Thus the map

$$
\begin{aligned}
& e: P \longrightarrow \mathbb{Q}^{\ell-k+1} \\
& e(\mathcal{P}):=\left(p_{\gamma^{1}}(\mathcal{P}), p_{\gamma^{2}}(\mathcal{P}), \ldots, p_{\gamma^{\ell-k+1}}(\mathcal{P})\right)
\end{aligned}
$$

is one to one. By a change of basis in $P$, and changing the order curves in $\gamma$ if necessary, we can assume that

$$
\pi_{i}\left(e\left(\mathcal{P}_{j}\right)\right)=\delta_{i j} \quad \forall 1 \leq i, j \leq b,
$$

where $\pi_{i}: \mathbb{Q}^{\ell-k+1} \rightarrow \mathbb{Q}$ is the projection over the $i$-th factor.

We would first like to show that for any positive periodic domain $\mathcal{Q}$ in $Q$, which is not included in the vector space generated by $A_{i}$ 's and $B_{j}$ 's, there is an integer $N=N(\mathcal{Q})$ such that by winding $\alpha$-curves $N$ times along the curves in $\gamma$ (in both positive and negative directions) the new periodic domain obtained from $\mathcal{Q}$ will have some negative coefficient. Let

$$
\mathcal{Q}=\sum_{i=1}^{b} q_{i} \mathcal{P}_{i}+\sum_{i=1}^{k} a_{i} A_{i}+\sum_{i=1}^{l} b_{i} B_{i}
$$

be a positive periodic domain in $Q$ such that there is an index $i$ so that $q_{i} \neq 0$. Then we may choose an integer $N$ such that

$$
\left|q_{i}\right| N>\max \left\{n_{v_{i}}(\mathcal{Q}), n_{\bar{v}_{i}}(\mathcal{Q})\right\} .
$$

Wind the $\alpha$ curves $N$ times along $\gamma$ curves. In the new diagram (obtained after the above winding procedure) let

$$
\left\{\mathcal{P}_{1}^{\prime}, \ldots, \mathcal{P}_{b}^{\prime}, A_{1}^{\prime}, \ldots, A_{k}^{\prime}, B_{1}^{\prime}, \ldots, B_{l}\right\}
$$

be the new set of periodic domains obtained from

$$
\left\{\mathcal{P}_{1}, \ldots, \mathcal{P}_{b}, A_{1}, \ldots, A_{k}, B_{1}, \ldots, B_{l}\right\}
$$

Note that we may compute the coefficients of these new domains at $v_{i}$ and $\bar{v}_{i}$ from the following equations

$$
\begin{aligned}
& n_{v_{i}}\left(A_{j}^{\prime}\right)=n_{v_{i}}\left(A_{j}\right), \quad \forall j=1, \ldots, k, \quad n_{v_{i}}\left(B_{j}^{\prime}\right)=n_{v_{i}}\left(B_{j}\right), \quad \forall j=1, \ldots, l, \\
& \qquad \quad n_{v_{i}}\left(\mathcal{P}_{j}^{\prime}\right)= \begin{cases}n_{v_{i}}\left(\mathcal{P}_{j}\right) & \text { if } i \neq j \\
n_{v_{i}}\left(\mathcal{P}_{j}\right)+N & \text { if } i=j\end{cases}
\end{aligned}
$$


Similar equations are satisfied for the local coefficients at $\bar{v}_{i}$. In fact, we will have $n_{\bar{v}_{i}}\left(\mathcal{P}_{i}^{\prime}\right)=n_{\bar{v}_{i}}\left(\mathcal{P}_{i}\right)-N$, while the rest of local coefficients remain unchanged. If $q_{i}<0$ we thus have

$$
n_{v_{i}}\left(\mathcal{Q}^{\prime}\right)=n_{v_{i}}(\mathcal{Q})+q_{i} N<0,
$$

and if $q_{i}>0$ then $n_{\bar{v}_{i}}\left(\mathcal{Q}^{\prime}\right)<0$.

To finish the proof, first suppose that there is an integer $N$ such that, after winding the $\alpha$ curves $N$ times along the curves in $\gamma$, any periodic domain $\mathcal{Q} \in Q$ with integer coefficients either has some negative coefficient or $\widetilde{\lambda}(\mathcal{Q})=0$. Then we are clearly done with the proof of the lemma. So, let us assume otherwise, that for any integer $n$ there exists a periodic domain $\mathcal{Q}_{n}$ with integer coefficients in $Q$ such that after winding the $\alpha$ curves $n$ times along the curves in $\gamma$, the resulting domain $\mathcal{Q}_{n}^{\prime}$ is positive and satisfies $\widetilde{\lambda}\left(\mathcal{Q}_{n}^{\prime}\right) \neq 0$. Let $\left\{\mathcal{Q}_{n}\right\}_{n=1}^{\infty}$ be the sequence constructed from these elements of $Q$. As in the proof of lemma 4.2. after passing to a subsequence if necessary, we may assume that the sequence $\left\{\frac{\mathcal{Q}_{n}}{\left\|\mathcal{Q}_{n}\right\|}\right\}_{n=1}^{\infty}$ is convergent.

Let us assume that

$$
\widetilde{\mathcal{Q}}=\lim _{i \rightarrow \infty} \frac{\mathcal{Q}_{n}}{\left\|\mathcal{Q}_{n}\right\|} \in Q \otimes_{\mathbb{Q}} \mathbb{R} .
$$

If $\widetilde{\mathcal{Q}}$ is not in the real vector space generated by $A_{i}$ 's and $B_{j}$ 's, there is an integer $N$ with the property that after winding the $\alpha$-curves $N$ times along all the curves in $\gamma$, the resulting domain $\widetilde{\mathcal{Q}}^{\prime}$ will have some negative coefficient. So there is an integer $K$ such that for any $i>K, \mathcal{Q}_{i}^{\prime}$ has some negative coefficient after winding the $\alpha$-curves $N$ times along $\gamma$. This is in contradiction with the definition of $\mathcal{Q}_{i}$ if $i>N$. Thus $\widetilde{\mathcal{Q}}$ may be written as

$$
\widetilde{\mathcal{Q}}=\sum_{i=1}^{k} a_{i} A_{i}+\sum_{i=1}^{l} b_{i} B_{i}
$$

for some coefficients $a_{i}$ and $b_{i}$ in $\mathbb{R}$.

Note that $\widetilde{\mathcal{Q}} \geq 0$, which implies that for any $w \in \Sigma$, we have

$$
\sum_{i=1}^{k} a_{i} n_{w}\left(A_{i}\right)+\sum_{i=1}^{l} b_{i} n_{w}\left(B_{i}\right) \geq 0 .
$$

Since $A_{i} \cap B_{j} \neq \emptyset$ for $i=1, \ldots, k$ and $j=1, \ldots, l$, we may pick $w=w_{i j}$ to be a point in this intersection. But for this choice of $w$, the above inequality reads as $a_{i}+b_{j} \geq 0$. If $b_{j}$ is the smallest of all $b_{1}, \ldots, b_{l}$, the above consideration implies that $a_{i}+b_{j} \geq 0$ for all $i=1, \ldots, k$. We may thus write

$$
\widetilde{\mathcal{Q}}=\sum_{i=1}^{k}\left(a_{i}+b_{j}\right) A_{i}+\sum_{i=1}^{l}\left(b_{i}-b_{j}\right) B_{i},
$$

since $\sum_{i} A_{i}=\sum_{i} B_{i}=\Sigma$. However, all the coefficients in the above expression are non-negative. As a result, after replacing these new coefficients, we may assume that the real numbers $a_{i}$ and $b_{j}$ in equation 7 are positive. 
As in the proof of lemma 4.2 , choose a positive rational periodic domain

$$
\mathcal{Q}=\sum_{i=1}^{k} a_{i}^{\prime} A_{i}+\sum_{j=1}^{l} b_{j}^{\prime} B_{j},
$$

which is sufficiently close to $\widetilde{\mathcal{Q}}$, and such that the coefficient of $\mathcal{Q} \in Q$ in the domains where $\widetilde{\mathcal{Q}}$ has zero coefficient is zero as well. As before, there are integers $N$ and $M$ such that $N \mathcal{Q}$ is a periodic domain with integer coefficients and $M \widetilde{\mathcal{Q}}-\mathcal{Q}>0$. The positivity of the coefficients of $N \mathcal{Q}$ imply that $\widetilde{\lambda}(N \mathcal{Q})=0$. Moreover, there is some positive integer $K>0$ such that for $i>K$ we have

$$
\begin{aligned}
& M N \frac{\mathcal{Q}_{i}}{\left\|\mathcal{Q}_{i}\right\|}-N \mathcal{Q} \geq 0, \& \frac{M}{\left\|\mathcal{Q}_{i}\right\|}<1 \\
\Rightarrow & N \mathcal{Q}_{i}-N \mathcal{Q} \geq 0 .
\end{aligned}
$$

This means that for $i>K$, we have $\widetilde{\lambda}\left(N \mathcal{Q}_{i}\right)=\widetilde{\lambda}\left(\mathcal{Q}_{i}\right)^{N}=0$. Thus we may conclude, using lemma 3.14 , that $\tilde{\lambda}\left(\mathcal{Q}_{i}\right)=0$ for $i>K$. This is in clear contradiction with our assumption on the integral periodic domains $\mathcal{Q}_{i}$.

The above argument shows that there is an integer $N$ with the property that after winding the curves in $\boldsymbol{\alpha}$ a total of $N$ times along the curves in $\gamma$ we obtain an $\mathfrak{s}$-admissible Heegaard diagram. This completes the proof of the lemma.

Remark 4.6. The argument of lemma 4.5 may be extended to show that for any balanced sutured manifold $(X, \tau)$ and any $\operatorname{Spin}^{c}$ class $\mathfrak{s} \in \operatorname{Spin}^{c}(\bar{X})$ there is a Heegaard diagram $(\Sigma, \boldsymbol{\alpha}, \boldsymbol{\beta}, \mathbf{z})$ which is admissible in the following stronger sense. If $\mathcal{P}$ is a periodic domain with $\mathcal{P} \geq 0$, and $\widetilde{\lambda}(\mathcal{P}) \neq 0$ in $\widetilde{\mathbb{A}}_{\tau}$ then

$$
\left\langle c_{1}(\mathfrak{s}), H(\mathcal{P})\right\rangle>0
$$

When there are genus zero components in $\mathfrak{R}(\tau)$, the above criteria is the same as $\mathfrak{s - a d m i s s i b i l i t y ~ c o n d i t i o n . ~ H o w e v e r , ~ i n ~ c e r t a i n ~ s i t u a t i o n s ~ w h e r e ~ a l l ~ t h e ~ c o n n e c t e d ~}$ components of $\mathfrak{R}(\tau)$ have positive genus, using such Heegaard diagrams may be useful. We face this situation in section 7 . 


\section{The CHAin COMPleX ASSOCIATED With A BALANCED SUTURED MANifold}

5.1. Holomorphic disks and boundary degenerations; orientation issues. Let us assume that $(\Sigma, \boldsymbol{\alpha}, \boldsymbol{\beta}, \mathbf{z})$ is an $\mathfrak{s}$-admissible Heegaard diagram for a balanced sutured manifold $(X, \tau)$ and $\mathfrak{s} \in \operatorname{Spin}^{c}\left(\bar{X}^{\tau}\right)$. We assume that $|\boldsymbol{\alpha}|=|\boldsymbol{\beta}|=\ell$ and that $\mathbf{z}=\left\{z_{1}, \ldots, z_{\kappa}\right\}$. We have already defined $\pi_{2}(\mathbf{x}, \mathbf{y})$ for any two intersection points $\mathbf{x}, \mathbf{y} \in \mathbb{T}_{\alpha} \cap \mathbb{T}_{\beta}$. In discussing the analytic aspects of a Floer theory, we need to consider boundary degenerations and sphere bubblings as well. We recall the following definitions from OS8.

Definition 5.1. Suppose that $\mathbf{x} \in \mathbb{T}_{\alpha} \cap \mathbb{T}_{\beta}$ is an arbitrary intersection point. $A$ continuous map

$$
\psi: \mathbb{R} \times[0, \infty) \longrightarrow \operatorname{Sym}^{\ell}(\Sigma)
$$

satisfying the boundary conditions

$$
\begin{gathered}
\psi(\mathbb{R} \times\{0\}) \subset \mathbb{T}_{\alpha}, \\
\lim _{|s| \rightarrow \infty} \psi(s, t)=\mathbf{x}, \quad \& \quad \lim _{t \rightarrow \infty} \psi(s, t)=\mathbf{x}
\end{gathered}
$$

is called an $\boldsymbol{\alpha}$-boundary degeneration. The space of homotopy classes of such maps is denoted by $\pi_{2}^{\alpha}(\mathbf{x})$. The space $\pi_{2}^{\beta}(\mathbf{x})$ of $\boldsymbol{\beta}$-boundary degenerations is defined similarly.

If $\left\{J_{t}=\operatorname{Sym}^{\ell}\left(j_{t}\right)\right\}_{t \in[0,1]}$ is a generic path of almost complex structure, associated with any $\phi \in \pi_{2}(\mathbf{x}, \mathbf{y})$ we may consider the moduli space $\mathcal{M}(\phi)$ of the representatives

$$
u:[0,1] \times \mathbb{R} \rightarrow \operatorname{Sym}^{\ell}(\Sigma)
$$

of $\phi$ which satisfy the time dependent Cauchy-Riemann equation

$$
\frac{\partial u}{\partial t}(t, s)+J_{t} \frac{\partial u}{\partial s}(t, s)=0, \quad \forall(t, s) \in[0,1] \times \mathbb{R} .
$$

Similarly, for any $\psi \in \pi_{2}^{\beta}(\mathbf{x}), \mathcal{N}(\psi)$ consists of the representatives $u:[0, \infty) \times \mathbb{R} \rightarrow$ $\operatorname{Sym}^{\ell}(\Sigma)$ of $\psi$ which are $J_{0}$-holomorphic. Also, for any $\psi \in \pi_{2}^{\alpha}(\mathbf{x}), \mathcal{N}(\psi)$ consists of the representatives $u:[0, \infty) \times \mathbb{R} \rightarrow \operatorname{Sym}^{\ell}(\Sigma)$ of $\psi$ which are $J_{1}$-holomorphic.

The determinant line bundle associated with the linearization of the (time dependent) Cauchy-Riemann operator over the moduli of representatives of any of the above homotopy classes is trivial. This makes it possible to equip the corresponding moduli space with an orientation. Following Ozsváth and Szabó's approach in OS5, we may choose a coherent system of orientations as follows.

As in the previous sections, let us assume that

$$
\Sigma-\boldsymbol{\alpha}=\coprod_{i=1}^{k} A_{i}, \quad \& \quad \Sigma-\boldsymbol{\beta}=\coprod_{j=1}^{l} B_{j},
$$

where $A_{i}$ and $B_{j}$ correspond to the components $R_{i}^{-} \subset \mathfrak{R}^{-}(\tau)$ and $R_{j}^{+} \subset \mathfrak{R}^{+}(\tau)$ respectively. Thus, the genus of $A_{i}$ is $g_{i}^{-}$and the genus of $B_{j}$ is $g_{j}^{+}$. Without loosing on generality, let us assume that $l \geq k$. Let $\mathbf{x}_{0}, \ldots, \mathbf{x}_{m}$ be all the intersection points in $\mathbb{T}_{\alpha} \cap \mathbb{T}_{\beta}$ which correspond to the $\operatorname{Spin}^{c}$ class $\mathfrak{s}$. Choose a disk class $\phi_{i} \in \pi_{2}\left(\mathbf{x}_{0}, \mathbf{x}_{i}\right)$ 
for each $i$, and complete the classes of the boundary degenerations $A_{1}, \ldots, A_{k}$ and $B_{1}, \ldots, B_{l-1}$ to a basis for the space of periodic domains in $\pi_{2}\left(\mathbf{x}_{0}, \mathbf{x}_{0}\right)$. Note that

$$
B_{l}=A_{1}+\ldots+A_{k}-\left(B_{1}+\ldots+B_{l-1}\right)
$$

is the only relation satisfied among $A_{1}, \ldots, A_{k}$ and $B_{1}, \ldots, B_{l}$. Let us denote this basis by $\psi_{1}, \ldots, \psi_{n}$. The choice of an orientation (i.e. one of the two classes represented by a non-vanishing section) on the determinant line bundle associated with the classes $\phi_{1}, \ldots, \phi_{m}$ and $\psi_{1}, \ldots, \psi_{n}$ induces an orientation on the moduli space corresponding to any class $\phi \in \pi_{2}\left(\mathbf{x}_{i}, \mathbf{x}_{j}\right), 0 \leq i, j \leq m$. In fact, $\phi+\phi_{i}-\phi_{j}$ is a periodic domain in $\pi_{2}\left(\mathbf{x}_{0}, \mathbf{x}_{0}\right)$, and is thus a linear combination of the classes $\psi_{1}, \ldots, \psi_{n}$. As a result, $\phi$ is a juxtaposition of (possibly several copies of) classes in

$$
\left\{\phi_{1}, \ldots, \phi_{m}, \psi_{1}, \ldots, \psi_{n}\right\},
$$

and thus inherits a natural orientation in our system of coherent orientations.

Let us study the boundary degenerations and their assigned orientation more carefully. Any periodic domain $\psi \in \pi_{2}(\mathbf{x}, \mathbf{x})$ such that $\partial \mathcal{D}(\psi)$ is a union of $\alpha$ curves determines the class of an $\alpha$ boundary degeneration. Thus, the domain of any $\alpha$ boundary degeneration $\psi \in \pi_{2}^{\alpha}(\mathbf{x})$ is a linear combination of $A_{1}, \ldots, A_{k}$ :

$$
\mathcal{D}(\psi)=a_{1} A_{1}+\ldots+a_{k} A_{k} .
$$

We may use Lipshitz' index formula to compute the Maslov index of $\psi$ :

$$
\mu(\psi)=a_{1} \chi\left(A_{1}\right)+\ldots+a_{k} \chi\left(A_{k}\right) .
$$

If furthermore, $\mathcal{D}(\psi)$ is a positive domain, e.g. if $\psi$ is a holomorphic boundary degeneration, then all $a_{i}$ are non-negative. We may then define the map

$$
\lambda: \coprod_{\mathbf{x} \in \mathbb{T}_{\alpha} \cap \mathbb{T}_{\beta}}\left(\pi_{2}^{\beta,+}(\mathbf{x}) \coprod \pi_{2}^{\alpha,+}(\mathbf{x})\right) \longrightarrow \mathbb{A}_{\tau}, \quad \lambda(\psi):=\prod_{i=1}^{\kappa} \lambda_{i}^{n_{z_{i}}(\psi)} .
$$

Here, we use $\pi_{2}^{\alpha,+}(\mathbf{x})$ (respectively, $\left.\pi_{2}^{\alpha,+}(\mathbf{x})\right)$ to denote the subset of $\pi_{2}^{\alpha}(\mathbf{x})$ (respectively, $\left.\pi_{2}^{\beta}(\mathbf{x})\right)$ which consists of the classes $\psi$ with $\mathcal{D}(\psi) \geq 0$.

If an $\alpha$ boundary degeneration $\psi$ as above is positive and $\lambda(\psi) \neq 0$, we may conclude that for $i=1, \ldots, k$, either $a_{i}=0$ or the genus of $A_{i}$ is zero. Without loosing on generality, assume that the genus of $A_{1}, \ldots, A_{k_{0}}$ is zero, and that the rest of $A_{i}$ have positive genus. Thus $\mathcal{D}(\psi) \geq 0$ and $\lambda(\psi) \neq 0$ implies that

$$
\mathcal{D}(\psi)=a_{1} A_{1}+\ldots+a_{k_{0}} A_{k_{0}} .
$$

Consequently $\mu(\psi)=2\left(a_{1}+\ldots+a_{k_{0}}\right)$. Similarly, we may assume that the genera of $B_{1}, \ldots, B_{l_{0}}$ are zero, and that the rest of $B_{i}$ have positive genus. This would imply that for any $\psi \in \pi_{2}^{\beta}(\mathbf{x})$ with $\mathcal{D}(\psi) \geq 0$, we will either have $\lambda(\psi)=0$, or

$$
\mathcal{D}(\psi)=b_{1} B_{1}+\ldots+b_{l_{0}} B_{l_{0}}, \quad \& \quad \mu(\psi)=2\left(b_{1}+\ldots+b_{l_{0}}\right) .
$$

In theorem 5.5 from OS8, Ozsváth and Szabó prove the following statement. In fact, the statement of their result is less general, but the proof applies in the following more general setup as well. 
Lemma 5.2. Let $\psi$ be the class of a boundary degeneration, and that a coherent choice of orientation is fixed for the Heegaard diagram $(\Sigma, \boldsymbol{\alpha}, \boldsymbol{\beta}, \mathbf{z})$. If $\mathcal{D}(\psi) \geq 0$, $\lambda(\psi) \neq 0$, and $\mu(\psi) \leq 2$ then $\mathcal{D}(\psi)=A_{i}$ or $\mathcal{D}(\psi)=B_{j}$ for some $1 \leq i \leq k_{0}$ or $1 \leq j \leq l_{0}$ (or $\psi$ is the class of the constant map). In the first case (i.e. $\mathcal{D}(\psi)=A_{i}$ ) we have

$$
\mathfrak{n}(\psi)= \begin{cases}0 & \text { if } k=1 \\ \pm 1 & \text { if } k>1 .\end{cases}
$$

where $\mathfrak{n}(\psi)=\# \widehat{\mathcal{N}}(\psi)$. Similarly, for $\mathcal{D}(\psi)=B_{j}$ we have $\mathfrak{n}(\psi)=0$ if $l=1$ and $\mathfrak{n}(\psi)= \pm 1$ if $l>1$. Here $\widehat{\mathcal{N}}(\psi)$ is the quotient of $\mathcal{N}(\psi)$ under the action of the subgroup

$$
\mathbb{G}=\left\{\left(\begin{array}{cc}
a & b \\
0 & \frac{1}{a}
\end{array}\right) \mid a \in \mathbb{R}^{+}, b \in \mathbb{R}\right\}<\mathrm{PSL}_{2}(\mathbb{R}) .
$$

Proof. See OS8] theorem 5.5. Note that the moduli spaces are now equipped with an orientation, and we may thus count the points of the moduli spaces with sign, instead of working modulo 2 . The choice of the plus or minus sign comes from the choice on the orientation associated with the homotopy classes $A_{i}$ and $B_{j}$ of $\alpha$ and $\beta$ boundary degenerations respectively.

The argument of Ozsváth and Szabó in fact implies that there is a natural choice of orientation for $A_{1}, \ldots, A_{k_{0}}$ and $B_{1}, \ldots, B_{l_{0}}$ which makes the value of $\mathfrak{n}(\psi)$ equal to +1 . This choice of orientation basically comes from the complex structure on the surface $\Sigma$, since the moduli space of boundary degenerations associated with any of $A_{1}, \ldots, A_{k_{0}}$ and $B_{1}, \ldots, B_{l_{0}}$ is eventually, possibly after stretching the necks, is identified with the group $\mathbb{G}$ via Riemann mapping theorem. This implies a form of compatibility among these preferred orientations on $A_{1}, \ldots, A_{k_{0}}$ and $B_{1}, \ldots, B_{l_{0}}$. More precisely, if $k=k_{0}$ and $l=l_{0}$, choosing the preferred orientation associated with $A_{1}, \ldots, A_{k}, B_{1}, \ldots, B_{l-1}$ induces an orientation on the moduli space corresponding to $B_{l}$. This orientation is the same as the preferred orientation associated with $B_{l}$. If $l_{0}<l$ (or if $k_{0}<k$ ), we are free to choose the preferred orientation associated with the classes $A_{1}, \ldots, A_{k_{0}}$ and $B_{1}, \ldots, B_{l_{0}}$ without any compatibility requirement. We may thus present the following definition.

Definition 5.3. A coherent system of orientations associated with the Heegaard diagram $(\Sigma, \boldsymbol{\alpha}, \boldsymbol{\beta}, \mathbf{z})$ for the balanced sutured manifold $(X, \tau)$ and the $\operatorname{Spin}^{c}$ class $\mathfrak{s} \in \operatorname{Spin}^{c}\left(\bar{X}^{\tau}\right)$ is an assignment $\mathfrak{o}$ of an orientation to the determinant line bundle of the linearized Cauchy-Riemann operator associated with all classes in $\pi_{2}(\mathbf{x}, \mathbf{y})$ (for all $\mathbf{x}, \mathbf{y} \in \mathbb{T}_{\alpha} \cap \mathbb{T}_{\beta}$ ) with the following properties:

- $\mathfrak{o}\left(\phi_{1} \star \phi_{2}\right)$ is the orientation induced by $\mathfrak{o}\left(\phi_{1}\right)$ and $\mathfrak{o}\left(\phi_{2}\right)$ via juxtaposition, for any $\mathbf{x}, \mathbf{y}, \mathbf{z} \in \mathbb{T}_{\alpha} \cap \mathbb{T}_{\beta}$ representing $\mathfrak{s}$, and any $\phi_{1} \in \pi_{2}(\mathbf{x}, \mathbf{y})$ and $\phi_{2} \in \pi_{2}(\mathbf{y}, \mathbf{z})$.

- For any $\mathrm{x} \in \mathbb{T}_{\alpha} \cap \mathbb{T}_{\beta}$ representing $\mathfrak{s}$, any $R_{i}^{-} \subset \mathfrak{R}^{-}(\tau)$ with $g_{i}^{-}=0$, and any $R_{j}^{+} \subset \mathfrak{R}^{+}(\tau)$ with $g_{j}^{+}=0$, let us denote by $\psi^{-} \in \pi_{2}^{\alpha}(\mathbf{x})$ and $\psi^{+} \in \pi_{2}^{\beta}(\mathbf{x})$ the classes of boundary degenerations corresponding to $R_{i}^{-}$and $R_{j}^{+}$respectively. Then $\mathfrak{o}\left(\psi^{-}\right)$and $\mathfrak{o}\left(\psi^{+}\right)$are the preferred orientation of Ozsváth and Szabó on $\mathcal{N}\left(\psi^{-}\right)$ 
and $\mathcal{N}\left(\psi^{+}\right)$respectively, which give $\mathfrak{n}\left(\psi^{-}\right)=1$ and $\mathfrak{n}\left(\psi^{+}\right)=1$ (if $k>1$ and $l>1$ respectively).

The last assumption implies, in particular, that the orientation induced on the periodic domain determined by $\Sigma$ is the natural orientation on it, as defined in section 3.6 of [OS5].

Let us assume that $\psi \in \pi_{2}^{\alpha}\left(\mathbf{x}_{0}\right)$ satisfies $\mathcal{D}(\psi) \in\left\{A_{1}, \ldots, A_{k_{0}}\right\}$, say $\mathcal{D}(\psi)=A_{1}$. Furthermore, assume that a preferred orientation on $\mathcal{N}(\psi)$ is fixed as before. At the same time $\psi$ may be regarded as a class in $\pi_{2}\left(\mathbf{x}_{0}, \mathbf{x}_{0}\right)$, and a moduli space $\mathcal{M}(\psi)$ may also be associated with $\psi$. This moduli space is smooth and two dimensional as well, and gives an open 1-manifold $\widehat{\mathcal{M}}(\psi)$ after we mod out by the translation action of $\mathbb{R}$. The choice of orientation on $\mathcal{N}(\psi)$ induces an orientation on $\mathcal{M}(\psi)$ as well. The reason is that the determinant line bundle of the (time dependent) Cauchy-Riemann operator on both these moduli spaces is pulled back from the same model, as discussed in subsection 3.6 in OS5].

According to the discussion of section 5 from OS8, $\widehat{\mathcal{N}}(\psi)$ will then appear as a boundary point of the smooth one dimensional manifold $\widehat{\mathcal{M}}(\psi)$. This induces a second orientation on $\widehat{\mathcal{N}}(\psi)$, as the boundary of the oriented moduli space $\widehat{\mathcal{M}}(\psi)$. Whether this second orientation agrees with the orientation of $\widehat{\mathcal{N}}(\psi)$ as the quotient of $\mathcal{N}(\psi)$ under the action of $\mathbb{G}$ or not depends on our convention for the embedding of the translation group $\mathbb{R}$ (which acts on on $\mathcal{M}(\psi)$ ) in $\mathbb{G}$, as will be discussed in more detail below. The same discussion is valid for $\beta$ boundary degenerations.

By the Riemann mapping theorem, the half plane

$$
\mathbb{H}^{+}=\mathbb{R} \times[0,+\infty) \subset \mathbb{C}
$$

is conformal to the unit disk, or to the strip $[0,1] \times \mathbb{R} \subset \mathbb{C}$. We may thus think of $\mathbb{H}^{+}$as the domain of the class $\psi$, when considered as an element in $\pi_{2}(\mathbf{x}, \mathbf{x})$. We may then fix a real number $r \in \mathbb{R}$ and interpret $\psi$ as a class with

$$
\psi([r,+\infty) \times\{0\}) \subset \mathbb{T}_{\alpha}, \quad \& \psi((-\infty, r] \times\{0\}) \subset \mathbb{T}_{\beta} .
$$

Furthermore, we have to assume that $\psi(r, 0)$ and $\psi(\infty)$ are both the intersection point $\mathbf{x} \in \mathbb{T}_{\alpha} \cap \mathbb{T}_{\beta}$. The group $\mathbb{G}$ consists of the maps $\rho_{a, b}$ for $a>0$ and $b \in \mathbb{R}$ which are defined by

$$
\rho_{a, b}(z):=a z+b .
$$

With this notation fixed, the re-parametrization group of the domain of $\psi \in \pi_{2}(\mathbf{x}, \mathbf{x})$ is then identified as

$$
\mathbb{R}_{r}=\left\{\rho_{a, b} \mid a \in \mathbb{R}^{+} \& b=r(1-a)\right\}<\mathbb{G} .
$$

If we identify $a$ as the exponential of a real number, the subgroup $\mathbb{R}_{r}$ is identified with $\mathbb{R}$. This induces an orientation on the one dimensional subgroup $\mathbb{R}_{r}$ of $\mathbb{G}$.

As $r$ approaches $-\infty$, the limit of the subgroups $\mathbb{R}_{r}$ determines the embedding of the translation group in the automorphism group of the domain of $\alpha$ boundary degenerations. In the this case, assuming $r<<0$ we may write

$$
a=1-\frac{c}{r}, \quad c \in(r, \infty), \quad \Rightarrow b=c .
$$


As $c$ grows large, $a$ grows large as well. Thus the above parametrization of $\mathbb{R}_{r}$ by the interval $(r,+\infty)$ is orientation preserving. With $r$ converging to $-\infty$, the sequence $\left\{\rho_{1-(c / r), c}\right\}_{r}$ converges to

$$
\rho_{1, c}: \mathbb{H}^{+} \rightarrow \mathbb{H}^{+}, \quad \rho_{1, c}(z)=z+c .
$$

The limit of $\mathbb{R}_{r}$, as $r \rightarrow-\infty$, is thus the translation subgroup

$$
\mathbb{R}_{\alpha}=\left\{\rho_{1, c} \mid c \in \mathbb{R}\right\}<\mathbb{G},
$$

and the above parametrization of $\mathbb{R}_{\alpha}$ is orientation preserving.

On the other hand, when $r$ approaches $+\infty$, the limit of the subgroups $\mathbb{R}_{r}$ determines the embedding of the translation group in the automorphism group of the domain of $\beta$ boundary degenerations. In the this later case, assuming $r>>0$ we may write

$$
a=1-\frac{c}{r}, \quad c \in(-\infty, r), \Rightarrow b=c .
$$

This time, as $c$ grows large, $a$ becomes small. Thus the above parametrization of $\mathbb{R}_{r}$ by the interval $(-\infty, r)$ is orientation reversing. With $r$ growing large, the sequence $\left\{\rho_{1-(c / r), c}\right\}_{r}$ converges to

$$
\rho_{1, c}: \mathbb{H}^{+} \rightarrow \mathbb{H}^{+}, \quad \rho_{1, c}(z)=z+c .
$$

The limit of $\mathbb{R}_{r}$, as $r \rightarrow+\infty$, is thus the translation subgroup

$$
\mathbb{R}_{\beta}=\left\{\rho_{1, c} \mid c \in \mathbb{R}\right\}<\mathbb{G},
$$

but this time, the above parametrization of $\mathbb{R}_{\beta}$ is orientation reversing.

With the above conventions for the orientations of $\mathbb{G}, \mathbb{R}_{r}, \mathbb{R}_{\alpha}$ and $\mathbb{R}_{\beta}$ fixed, we have thus proved the following lemma.

Lemma 5.4. Let $\phi \in \pi_{2}^{\alpha}(\mathbf{x})$ and $\psi \in \pi_{2}^{\beta}(\mathbf{x})$ be the classes of $\alpha$ and $\beta$ boundary degenerations respectively. Furthermore, assume that $\mu(\phi)=\mu(\psi)=2$, and that $\mathcal{N}(\phi)$ and $\mathcal{N}(\psi)$ are smooth manifolds. Then the orientation induced on $\widehat{\mathcal{N}}(\phi)$ agrees with the boundary orientation induced from $\widehat{\mathcal{M}}(\phi)$, while the orientation induced on $\widehat{\mathcal{N}}(\psi)$ is the opposite of the boundary orientation induced from $\widehat{\mathcal{M}}(\psi)$.

5.2. Energy bounds and relative gradings. Recall that for a Riemannian manifold $(M, g)$ and a domain $\Omega \subset \mathbb{C}$ the energy of a smooth map $u: \Omega \rightarrow X$ is defined by

$$
E(u)=\frac{1}{2} \int_{\Omega}\|d u\|_{g}^{2}
$$

Suppose that $(\Sigma, \boldsymbol{\alpha}, \boldsymbol{\beta}, \mathbf{z})$ is a Heegaard diagram for a balanced sutured manifold $(X, \tau)$. Let

$$
\Sigma-\boldsymbol{\alpha}-\boldsymbol{\beta}=\coprod_{i=1}^{m} D_{i}
$$

be the connected components in the complement of the curves, and $\eta$ denotes a Kähler form on $\Sigma$. We denote the area of $D_{i}$ with respect to $\eta$ by $\operatorname{Area}_{\eta}\left(D_{i}\right)$, and for a domain $\mathcal{D}=\sum_{i=1}^{m} a_{i} D_{i}$ we define

$$
\operatorname{Area}_{\eta}(\mathcal{D})=\sum_{i=1}^{m} a_{i} \operatorname{Area}_{\eta}\left(D_{i}\right)
$$


The following lemma is basically lemma 3.5 from [OS5] and theorem 6.3 from [Ju1.

Lemma 5.5. There is a constant $C$ which depends only on the Heegaard diagram $(\Sigma, \boldsymbol{\alpha}, \boldsymbol{\beta}, \mathbf{z})$ and the Kähler form $\eta$ such that for any pseudo-holomorphic Whitney disk

we have

$$
u:(\mathbb{D}, \partial \mathbb{D}) \longrightarrow\left(\operatorname{Sym}^{\ell}(\Sigma), \mathbb{T}_{\alpha} \cup \mathbb{T}_{\beta}\right)
$$

$$
E(u) \leq C \cdot \operatorname{Area}_{\eta}(\mathcal{D}(u)) .
$$

The existence of energy bounds is needed in Gromov compactness arguments.

Finally, note that

$$
\pi_{1}\left(\operatorname{Sym}^{\ell}(\Sigma)\right)=\mathrm{H}_{1}\left(\operatorname{Sym}^{\ell}(\Sigma) ; \mathbb{Z}\right)
$$

provided that $\ell>1$. Throughout the construction, we will assume that the requirement $\ell>1$ is satisfied, by stabilizing Heegaard diagrams if necessary.

Definition 5.6. For $\mathfrak{s} \in \operatorname{Spin}^{c}(\bar{X})$ let

$$
\mathfrak{d}(\mathfrak{s})=\operatorname{gcd}_{h \in \mathrm{H}_{2}(\bar{X}, \mathbb{Z})}\left\langle c_{1}(\mathfrak{s}), h\right\rangle .
$$

If $H=(\Sigma, \boldsymbol{\alpha}, \boldsymbol{\beta}, \mathbf{z})$ is an $\mathfrak{s}$-admissible Heegaard diagram for $(X, \tau)$ for any $\mathbf{x}, \mathbf{y} \in$ $\mathbb{T}_{\alpha} \cap \mathbb{T}_{\beta}$ with $\mathfrak{s}(\mathbf{x}), \mathfrak{s}(\mathbf{y}) \in \mathfrak{s}$, and for $\phi \in \pi_{2}(\mathbf{x}, \mathbf{y})$, we define the relative grading of $\mathbf{x}$ and $\mathbf{y}$ by

Thus, $\operatorname{gr}(\mathbf{x}, \mathbf{y}) \in \mathbb{Z}_{\mathfrak{d}(\mathfrak{s})}=\frac{\mathbb{Z}}{\mathfrak{d}(\mathfrak{s}) \mathbb{Z}}$.

$$
\operatorname{gr}(\mathbf{x}, \mathbf{y})=\mu(\phi) \quad(\bmod \mathfrak{d}(\mathfrak{s}))
$$

The relative grading is independent of the choice of $\phi$. It induces a relative grading on the module $\mathrm{CF}(X, \tau, \mathfrak{s} ; H)$. For this purpose, we should determine the grading associated with the generators $\lambda_{1}, \ldots, \lambda_{\kappa} \in G(\mathbb{A})$. Each $\lambda_{i}$ corresponds to the class

$$
\left[\gamma_{i}\right] \in \operatorname{Ker}\left(\left(\imath_{X}\right)_{*}: \mathrm{H}_{1}(X ; \mathbb{Z}) \rightarrow \mathrm{H}_{1}(\bar{X} ; \mathbb{Z})\right),
$$

where $\imath_{X}: X \rightarrow \bar{X}$ is the inclusion map. It is thus the boundary of an integral 2-chain $A_{i}=A_{\left[\gamma_{i}\right]}$ in $\bar{X}$, which is well defined up to addition of 2-cycles. The evaluation $d_{i}=-\left\langle c_{1}(\mathfrak{s}), A_{i}\right\rangle$ is thus well-defined as an element of $\frac{\mathbb{Z}}{\mathfrak{d}(\mathfrak{s}) \mathbb{Z}}$. We may then define the grading on $G(\mathbb{A})$ by setting

$$
\operatorname{gr}\left(\prod_{i=1}^{\kappa} \lambda_{i}^{n_{i}}\right):=\sum_{i=1}^{\kappa} d_{i} n_{i} \in \frac{\mathbb{Z}}{\mathfrak{d}(\mathfrak{s}) \mathbb{Z}}, \quad \forall \prod_{i=1}^{\kappa} \lambda_{i}^{n_{i}} \in G(\mathbb{A}) .
$$

If $\phi \in \pi_{2}(\mathbf{x}, \mathbf{x})$ is a positive disk, it determines a periodic domain, and

$$
\mu(\phi)=\left\langle c_{1}(\mathfrak{s}), H(\phi)\right\rangle=\operatorname{gr}\left(\lambda_{\mathbf{z}}(\phi)\right) \quad(\bmod \mathfrak{d}(\mathfrak{s})) .
$$

The $\mathbb{A}$-module $\operatorname{CF}(X, \tau, \mathfrak{s} ; H)$ is thus equipped with a relative homological grading by the elements in $\frac{\mathbb{Z}}{\mathfrak{d}(\mathfrak{s}) \mathbb{Z}}$. The differential of the corresponding Ozsváth-Szabó complex $\mathrm{CF}(X, \tau, \mathfrak{s} ; H)$ which will be defined in the following subsection lowers this relative grading by one. In particular, a relative grading is induced on the homology groups corresponding to any test ring $\mathbb{B}$ for $\mathbb{A}_{\tau}$. 
5.3. The construction of the chain complex. Let $(X, \tau)$ be a balanced sutured manifold. As discussed in section 3 we associate a coefficient ring $\mathbb{A}=\mathbb{A}_{\tau}$ with $\tau$, which is a $\mathbb{Z}$-algebra. Let us denote by $\bar{X}$ the three-manifold (with positive and negative boundary components) obtained from $X$ by filling out the sutures in $\tau$. Let $\mathfrak{s} \in \operatorname{Spin}^{c}(\bar{X})$ be a $\operatorname{Spin}^{c}$ structure on $\bar{X}$. Consider an $\mathfrak{s}$-admissible Heegaard diagram $(\Sigma, \boldsymbol{\alpha}, \boldsymbol{\beta}, \mathbf{z})$ for $(X, \tau)$. Associated with this Heegaard diagram, let

$$
\mathrm{CF}(\Sigma, \boldsymbol{\alpha}, \boldsymbol{\beta}, \mathbf{z} ; \mathfrak{s})=\left\langle\mathbf{x} \in \mathbb{T}_{\alpha} \cap \mathbb{T}_{\beta} \mid \underline{\mathfrak{s}}_{\mathbf{z}}(\mathbf{x}) \in \mathfrak{s}\right\rangle_{\mathbb{A}}
$$

be a free $\mathbb{A}$-module generated by the intersection points in $\mathbb{T}_{\alpha} \cap \mathbb{T}_{\beta}$ which represent the $\operatorname{Spin}^{c}$ class $\mathfrak{s}$. Note that the "exact sequence"

$$
0 \longrightarrow\left\langle\operatorname{PD}\left[\gamma_{i}\right]\right\rangle_{i=1}^{\kappa} \longrightarrow \operatorname{Spin}^{c}(X, \tau) \stackrel{[.]=s_{\{1, \ldots, \kappa\}}}{\longrightarrow} \operatorname{Spin}^{c}(\bar{X}) \longrightarrow 0
$$

implies that the assignment of relative $\operatorname{Spin}^{c}$ structures gives a filtration on the $\mathbb{A}$-module $\operatorname{CF}(\Sigma, \boldsymbol{\alpha}, \boldsymbol{\beta}, \mathbf{z} ; \mathfrak{s})$, which is compatible with the filtration $\chi: G(\mathbb{A}) \rightarrow \mathbb{H}$. This module may be decomposed using the filtration by relative $\operatorname{Spin}^{c}$ structures:

$$
\begin{aligned}
& \mathrm{CF}(\Sigma, \boldsymbol{\alpha}, \boldsymbol{\beta}, \mathbf{z} ; \mathfrak{s})=\bigoplus_{\mathfrak{s} \in \mathfrak{s} \subset \operatorname{Spin}^{c}(X, \tau)} \mathrm{CF}(\Sigma, \boldsymbol{\alpha}, \boldsymbol{\beta}, \mathbf{z} ; \mathfrak{\mathfrak { s }}) \\
& \mathrm{CF}(\Sigma, \boldsymbol{\alpha}, \boldsymbol{\beta}, \mathbf{z} ; \mathfrak{\mathfrak { s }})=\left\langle\lambda \mathbf{x} \mid \mathbf{x} \in \mathbb{T}_{\alpha} \cap \mathbb{T}_{\beta}, \lambda \in G(\mathbb{A}), \quad \& \mathfrak{\mathfrak { s }}_{\mathbf{z}}(\mathbf{x})+\chi(\lambda)=\mathfrak{s}\right\rangle_{\mathbb{Z}}
\end{aligned}
$$

Here $G(\mathbb{A})$ denotes the set of generator of the form $\lambda=\prod_{i=1}^{\kappa} \lambda_{i}^{a_{i}}$ for $\mathbb{A}$, as a module over $\mathbb{Z}$.

Furthermore, fix a coherent system $\mathfrak{o}$ of orientations on the determinant line bundles of the linearization of Cauchy-Riemann operators associated with the classes of the Whitney disks (corresponding to $\mathfrak{s}$ ). We will drop $\mathfrak{o}$ from the notation, unless an issue related to the orientation should be discussed.

Define an $\mathbb{A}$-module homomorphism by the following equation

$$
\begin{aligned}
& \partial: \mathrm{CF}(\Sigma, \boldsymbol{\alpha}, \boldsymbol{\beta}, \mathbf{z} ; \mathfrak{s}) \longrightarrow \mathrm{CF}(\Sigma, \boldsymbol{\alpha}, \boldsymbol{\beta}, \mathbf{z} ; \mathfrak{s}) \\
& \partial(\mathbf{x}):=\sum_{y \in \mathbb{T}_{\alpha} \cap \mathbb{T}_{\beta}} \sum_{\left\{\phi \in \pi_{2}^{+}(\mathbf{x}, \mathbf{y}) \mid \mu(\phi)=1\right\}}(\mathfrak{m}(\phi) \lambda(\phi)) . \mathbf{y} .
\end{aligned}
$$

Here $\mathfrak{m}(\phi)=\# \widehat{\mathcal{M}}(\phi)$ is the algebraic count (i.e. with the signs determined by the orientation) of the points in $\widehat{\mathcal{M}}(\phi)$ for any positive Whitney disk class $\phi \in \pi_{2}(\mathbf{x}, \mathbf{y})$ such that $\lambda(\phi) \neq 0$. For other disk classes, the contribution $\mathfrak{m}(\phi) \lambda(\phi)$ is trivial by definition.

It is important to note that for any $\phi \in \pi_{2}^{+}(\mathbf{x}, \mathbf{y})$ with $\mu(\phi)=1$ and $\lambda(\phi) \neq$ 0 , the moduli space $\widehat{\mathcal{M}}(\phi)$ is smooth, zero dimensional, oriented, and compact. Smoothness and zero dimensionality of the moduli space follows from the generic choice of the path of complex structures on $\operatorname{Sym}^{\ell}(\Sigma)$ (see the general discussion of OS5, section 3). The compactness is however more critical. If $u_{i}$ is a sequence of pseudo-holomorphic representatives of $\phi$, the amount of energy $E\left(u_{i}\right)$ remains bounded by lemma 5.5. We may thus use the Gromov compactness theorem to describe the possible limits of this sequence. In fact, any possible Gromov limit of the sequence is the juxtaposition of some pseudo-holomorphic representative $u$ 
of a class $\phi^{\prime} \in \pi_{2}^{+}(\mathbf{x}, \mathbf{y})$ with boundary degenerations and sphere bubblings. Let us assume that $v_{1}, \ldots, v_{p}$ are the classes of degenerations and bubbles. Then the domains of $u$ and $v_{i}, i=1, \ldots, p$ are positive and

$$
\lambda(\phi)=\lambda(u) \lambda\left(v_{1}\right) \ldots \lambda\left(v_{p}\right) \neq 0 .
$$

This implies that the domain of each $v_{i}$ is a linear combination of the domains $A_{1}, \ldots, A_{k_{0}}$ or $B_{1}, \ldots, B_{l_{0}}$ (with non-negative coefficients). Here $A_{1}, \ldots, A_{k_{0}}$ are the zero genus components in $\Sigma-\boldsymbol{\alpha}$ and $B_{1}, \ldots, B_{l_{0}}$ are the zero genus components in $\Sigma-\boldsymbol{\beta}$. The Maslov index of each $v_{i}$ is thus a positive even number. Since the $\mathcal{M}\left(\phi^{\prime}\right) \neq \emptyset, \mu\left(\phi^{\prime}\right)$ is non-negative and $p$ is thus forced to be 0 . However, this means that the Gromov limit of $u_{i}$ is in $\widehat{\mathcal{M}}(\phi)$, i.e. $\widehat{\mathcal{M}}(\phi)$ is compact, and thus finite. In other words, for any class $\phi \in \pi_{2}^{+}(\mathbf{x}, \mathbf{y})$ with $\mu(\phi)=1$, either $\phi$ does not contribute to the coefficient of $\mathbf{y}$ in $\partial(\mathbf{x})$ (e.g. $\lambda(\phi)=0)$, or $\mathfrak{m}(\phi)$ is finite.

The Heegaard diagram $(\Sigma, \boldsymbol{\alpha}, \boldsymbol{\beta}, \mathbf{z} ; \mathfrak{s})$ is $\mathfrak{s}$-admissible, so by lemma 4.5 for any intersection point $\mathbf{y} \in \mathbb{T}_{\alpha} \cap \mathbb{T}_{\beta}$ there are only finitely many $\phi \in \pi_{2}(\mathbf{x}, \mathbf{y})$ such that $\mu(\phi)=1, \mathcal{D}(\phi) \geq 0$, and $\lambda(\phi) \neq 0$. Thus there are only finitely many classes $\phi \in \pi_{2}^{+}(\mathbf{x}, \mathbf{y})$ with $\mu(\phi)=1$ and $\lambda(\phi) \neq 0$ which admit holomorphic representative. This shows that the terms which contribute to the coefficient of $\mathbf{y}$ in $\partial(\mathbf{x})$ are finite, and that the map $\partial$ is thus well-defined.

The map $\partial$ is, by definition, a homomorphism of $\mathbb{A}$-modules. It is obvious from the definition and the discussion of subsection 3.4 that $\partial$ preserves the decomposition of equation 8 , and we thus obtain a set of $\mathbb{Z}$-module homomorphisms

$$
\partial: \mathrm{CF}(\Sigma, \boldsymbol{\alpha}, \boldsymbol{\beta}, \mathbf{z} ; \underline{\mathfrak{s}}) \longrightarrow \mathrm{CF}(\Sigma, \boldsymbol{\alpha}, \boldsymbol{\beta}, \mathbf{z} ; \underline{\mathfrak{s}}),
$$

for any relative $\operatorname{Spin}^{c}$ class $\underline{\mathfrak{s}} \in \operatorname{Spin}^{c}(X, \tau)$.

Theorem 5.7. The filtered $\mathbb{A}$-module $\mathrm{CF}(\Sigma, \boldsymbol{\alpha}, \boldsymbol{\beta}, \mathbf{z} ; \mathfrak{s})$ is a filtered $(\mathbb{A}, \mathbb{H})$ chain complex, where $\mathbb{A}=\mathbb{A}_{\tau}$ is the coefficient ring associated with $\tau$ and the filtration by the elements of the $\mathbb{Z}$-module $\mathbb{H}=\mathrm{H}^{2}(X, \partial X ; \mathbb{Z})$ is given by the assignment of the relative $\operatorname{Spin}^{c}$ classes in $\operatorname{Spin}^{c}(X, \tau)$ to the generators in $\mathbb{T}_{\alpha} \cap \mathbb{T}_{\beta}$ using the map $\underline{\underline{\mathfrak{y}}}_{\mathbf{z}}$.

Before we start proving the above theorem we re-phrase lemma 5.2 in the presence of a coherent system of orientation.

Lemma 5.8. With the notation of lemma 5.2 fixed, let $\mathfrak{o}$ be a coherent system of orientations associated with the Heegaard diagram. Let $\psi$ be the class of a boundary degeneration. If $\mathcal{D}(\psi) \geq 0, \lambda(\psi) \neq 0$, and $\mu(\psi) \leq 2$ then $\mathcal{D}(\psi)=A_{i}$ or $\mathcal{D}(\psi)=B_{j}$ for some $1 \leq i \leq k_{0}$ or $1 \leq j \leq l_{0}$ (or $\psi$ is the class of the constant map). In the first case $\left(\right.$ i.e. $\left.\mathcal{D}(\psi)=A_{i}\right)$ we have

$$
\mathfrak{n}(\psi)= \begin{cases}0 & \text { if } k=1 \\ 1 & \text { if } k>1\end{cases}
$$

Similarly, for $\mathcal{D}(\psi)=B_{j}$ we have $\mathfrak{n}(\psi)=0$ if $l=1$ and $\mathfrak{n}(\psi)=1$ if $l>1$.

Now we can prove theorem 5.7 using the above lemma. The proof is similar to the proof of lemma 4.3 in OS8.

Proof. (of Theorem 5.7.) Clearly, for any Whitney disk $\phi \in \pi_{2}(\mathbf{x}, \mathbf{y})$, which has a holomorphic representative, we have $\mathcal{D}(\phi) \geq 0$ and $\lambda(\phi)$ is thus a well-defined 
element of $\mathbb{A}$. Thus we only need to prove $\partial \circ \partial=0$.

Let $\mathbf{x}$ and $\mathbf{y}$ be two intersection points in $\mathbb{T}_{\alpha} \cap \mathbb{T}_{\beta}$. Fix a class $\phi \in \pi_{2}(\mathbf{x}, \mathbf{y})$ such that $\mu(\phi)=2$. Consider the ends of the moduli space $\widehat{\mathcal{M}}(\phi)$. This space has three types of ends, which are in correspondence with the broken flow-lines. More precisely, these are (respectively) the ends corresponding to a holomorphic Whitney disk $\phi_{1}$ connecting $\mathbf{x}$ to an intersection point $\mathbf{w}$ juxtaposed with a holomorphic Whitney disk $\phi_{2}$ connecting $\mathbf{w}$ to $\mathbf{y}$ such that $\mu\left(\phi_{1}\right)=\mu\left(\phi_{2}\right)=1$, the ends corresponding to a sphere bubbling off i.e. a holomorphic Whitney disk $\phi^{\prime}$ connecting $\mathbf{x}$ to $\mathbf{y}$ juxtaposed with a holomorphic sphere $S$ in $\operatorname{Sym}^{\ell}(\Sigma)$, and the ends corresponding to a boundary bubbling i.e. a holomorphic Whitney disk $\phi^{\prime}$ connecting $\mathbf{x}$ to $\mathbf{y}$ juxtaposed with a holomorphic boundary degeneration.

If $\mathbf{x} \neq \mathbf{y}$ the space $\widehat{\mathcal{M}}(\phi)$ does not have any boundary of the second and the third types, since any holomorphic boundary degeneration or holomorphic sphere with the property that its associated monomial in $\mathbb{A}$ is non-trivial will have Maslov index at least 2. Thus the remaining Whitney disk should have Maslov index less than or equal to zero. This implies that the moduli space associated with the Whitney disk is empty, or consists of a constant function (which can not happen by the assumption $\mathbf{x} \neq \mathbf{y}$ ). When $\mathbf{x} \neq \mathbf{y}$ the Gromov ends of this moduli space thus consist of

$$
\coprod_{\mathbf{w} \in \mathbb{T}_{\alpha} \cap \mathbb{T}_{\beta}} \coprod_{\substack{\phi_{1} \in \pi_{2}(\mathbf{x}, \mathbf{w}) \\ \phi_{2} \in \pi_{2}(\mathbf{w}, \mathbf{y}) \\ \phi_{1} * \phi_{2}=\phi}}\left(\widehat{\mathcal{M}}\left(\phi_{1}\right) \times \widehat{\mathcal{M}}\left(\phi_{2}\right)\right)
$$

For any fixed $\lambda \in G(\mathbb{A})$ the coefficient of $\lambda \mathbf{y}$ in $\partial^{2} \mathbf{x}$ (assuming $\mathbf{x} \neq \mathbf{y}$ ) is equal to

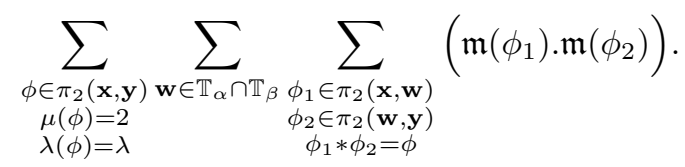

For each $\phi \in \pi_{2}(\mathbf{x}, \mathbf{y})$ the amount of the two interior sums in the above formula is the total number, counted with the sign determined by the coherent system $\mathfrak{o}$ of orientations, of the ends of the moduli space $\widehat{\mathcal{M}}(\phi)$, which is zero. Consequently the total sum in the above formula is trivial, and the coefficient of $\lambda \mathbf{y}$ in $\partial(\partial(\mathbf{x}))$ is thus equal to 0 .

Let us now assume that $\mathbf{x}=\mathbf{y}$. Let us denote the class of the generator of holomorphic spheres by $S$. The domain $\mathcal{D}(S)$ associated with $S$ is the surface $\Sigma$ :

$\mathcal{D}(S)=A_{1}+\ldots+A_{k}=B_{1}+\ldots+B_{l}, \quad \Rightarrow \lambda(S)=\lambda\left(A_{1}\right) \ldots \lambda\left(A_{k}\right)=\lambda\left(B_{1}\right) \ldots \lambda\left(B_{l}\right)$.

Thus $\lambda(S)=0$ unless $k=k_{0}=l_{0}=l$. In this later case, the Maslov index of $S$ is $2 k$, which is greater than 2 unless $k=1$. Combining with lemma 5.8, we may thus conclude that in all possible cases, the total contribution to $\partial^{2}(\mathbf{x})$ from sphere bubblings is trivial.

We may thus assume, without loosing on generality, that the ends of the moduli space $\widehat{\mathcal{M}}(\phi)$ do not contain any sphere bubblings. If the ends of this moduli space contain a boundary disk degeneration, then the degeneration would consist of the juxtaposition of a constant function and a holomorphic boundary degeneration with 
Maslov index 2. If we denote the boundary degeneration by $\psi$, lemma 5.8 implies that $\mathcal{D}(\psi)=A_{i}$ or $\mathcal{D}(\psi)=B_{j}$.

In the above situation, if $\mathcal{D}(\phi)=\mathcal{D}(\psi)=A_{i}$ or $B_{j}$, the boundary disk degeneration among the ends of $\widehat{\mathcal{M}}(\psi)$ are described by lemma 5.8. Suppose first that $k, l>1$. Let $\mathcal{D}(\phi)=B_{j}$, and let $\psi$ be the corresponding boundary disk degeneration with the same domain. Then the ends of $\mathcal{M}(\phi)$ consist of

$$
\widehat{\mathcal{N}}(\psi) \bigcup\left(\coprod_{\mathbf{w} \in \mathbb{T}_{\alpha} \cap \mathbb{T}_{\beta}} \coprod_{\substack{\phi_{1} \in \pi_{2}(\mathbf{x}, \mathbf{w}) \\ \phi_{2} \in \pi_{2}(\mathbf{w}, \mathbf{x}) \\ \phi_{1} \star \phi_{2}=\phi}}\left(\widehat{\mathcal{M}}\left(\phi_{1}\right) \times \widehat{\mathcal{M}}\left(\phi_{2}\right)\right)\right) .
$$

According to lemma 5.4 , the orientation of $\widehat{\mathcal{N}}(\psi)$ agrees with the orientation induced from $\widehat{\mathcal{M}}(\psi)$. Thus the total number of the ends for this moduli space is equal to

$$
\mathfrak{n}(\psi)+\sum_{\mathbf{w} \in \mathbb{T}_{\alpha} \cap \mathbb{T}_{\beta}} \sum_{\substack{\phi_{1} \in \pi_{2}(\mathbf{x}, \mathbf{w}) \\ \phi_{2} \in \pi_{2}(\mathbf{w}, \mathbf{x}) \\ \phi_{1} \star \phi_{2}=\phi}}\left(\mathfrak{m}\left(\phi_{1}\right) \cdot \mathfrak{m}\left(\phi_{2}\right)\right)=0 .
$$

By lemma 5.8 we have $\mathfrak{n}(\psi)=1$, thus the total value of the second sum is equal to -1 .

Note that $\lambda(\psi)=\lambda\left(R_{j}^{+}\right)=\lambda_{j}^{+}$, since the domain associated with $\psi$ is $B_{j}$. Thus, such degenerations contribute to the coefficient of $\lambda\left(B_{j}\right) \mathbf{x}$, i.e. the contribution of $\psi$ to $\partial^{2}(\mathbf{x})$ is $-\lambda_{j}^{+}$.x. Similarly, for a $\alpha$ boundary degeneration $\psi$ with $\mathcal{D}(\psi)=A_{i}$, we obtain the equality

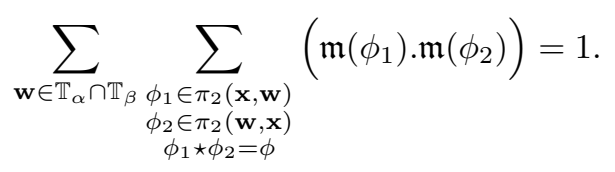

Thus the contribution of $\psi$ to $\partial^{2}(\mathbf{x})$ is $\lambda_{i}^{-} \cdot \mathbf{x}$.

The coefficient of $\mathbf{x}$ in $\partial(\partial(\mathbf{x}))$ is equal to

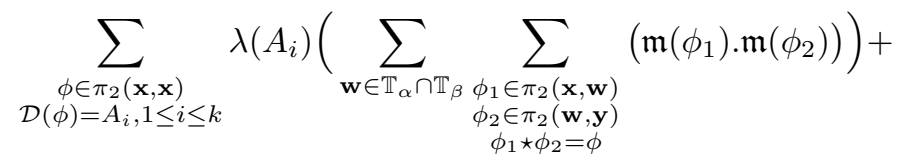

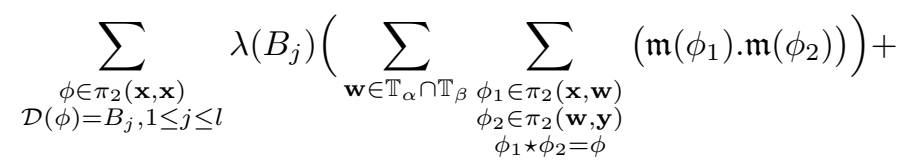

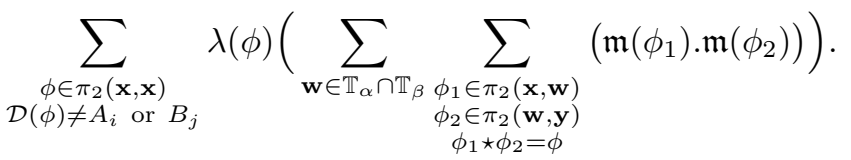

Our argument shows that in the above the sum, the sums in the first and the second line combine to give the following expression:

$$
\sum_{1 \leq i \leq k} \lambda\left(A_{i}\right)-\sum_{1 \leq i \leq l} \lambda\left(B_{i}\right)=\sum_{1 \leq i \leq k} \lambda\left(R_{i}^{+}\right)-\sum_{1 \leq i \leq l} \lambda\left(R_{i}^{-}\right)=0 .
$$


Thus the sum of the contributions from the first two lines in the above expression is zero. The last line is a sum of zero terms, by an argument similar to the case $\mathbf{x} \neq \mathbf{y}$, so it is trivial. Consequently, the coefficient of $\mathbf{x}$ in $\partial(\partial(\mathbf{x}))$ is zero.

When $k=1$ or $l=1$ one should be cautious. If $k=l=1$, the contributions from both $\alpha$ and $\beta$ boundary degenerations is zero by lemma 5.8. However, if $k=1$ and $l>1$, since the Heegaard diagram is balanced, we may conclude that $l>l_{0}$. However,

$$
\lambda\left(A_{1}\right)=\lambda(\Sigma)=\prod_{i=1}^{l} \lambda\left(B_{i}\right)=0 \Rightarrow \sum_{i=1}^{l} \lambda_{i}^{+}=\lambda_{1}^{-}=0 .
$$

The rest of the argument in this case if completely identical with the case $k, l>1$. This completes the proof of the theorem.

In the following section we will prove the following theorem.

Theorem 5.9. The filtered $(\mathbb{A}, \mathbb{H})$ chain homotopy type of the filtered $(\mathbb{A}, \mathbb{H})$ chain complex $\mathrm{CF}(\Sigma, \boldsymbol{\alpha}, \boldsymbol{\beta}, \mathbf{z} ; \mathfrak{s})$ is an invariant of the balanced sutured manifold and the $\operatorname{Spin}^{c}$ class $\mathfrak{s} \in \operatorname{Spin}^{c}(\bar{X})$. In particular, for any filtered test ring $\mathbb{B}$ for $(\mathbb{A}, \mathbb{H})$ and for any $\mathfrak{\underline { s }} \in \operatorname{Spin}^{c}(X, \tau)$, the chain homotopy type of

$$
\mathrm{CF}(\Sigma, \boldsymbol{\alpha}, \boldsymbol{\beta}, \mathbf{z} ; \mathfrak{\mathfrak { s }} ; \mathbb{B}) \subset \mathrm{CF}(\Sigma, \boldsymbol{\alpha}, \boldsymbol{\beta}, \mathbf{z} ; \mathfrak{s}) \otimes_{\mathbb{A}} \mathbb{B}
$$

is also an invariant of $(X, \tau, \underline{\mathfrak{s}})$.

Definition 5.10. We may thus denote the filtered $(\mathbb{A}, \mathbb{H})$ chain homotopy type of the filtered $(\mathbb{A}, \mathbb{H})$ chain complex $\mathrm{CF}(\Sigma, \boldsymbol{\alpha}, \boldsymbol{\beta}, \mathbf{z} ; \mathfrak{s})$ and its invariant decomposition into chain complexes $\mathrm{CF}(\Sigma, \boldsymbol{\alpha}, \boldsymbol{\beta}, \mathbf{z} ; \mathfrak{\mathfrak { s }})$ by

$$
\mathrm{CF}(X, \tau ; \mathfrak{s})=\bigoplus_{\underline{\mathfrak{s}} \in \mathfrak{s} \subset \operatorname{Spin}^{c}(X, \tau)} \mathrm{CF}(X, \tau ; \mathfrak{\mathfrak { s }}) .
$$

5.4. Additional algebraic structure. From the definitions, it is clear that the multiplication by a generator $\lambda \in G(\mathbb{A})$ gives a map

$$
m_{\lambda}: \mathrm{CF}(X, \tau ; \underline{\mathfrak{s}}) \longrightarrow \mathrm{CF}(X, \tau ; \underline{\mathfrak{s}}+\chi(\lambda)) .
$$

This map shifts the homological grading by $\operatorname{gr}(\lambda)$, and generalizes the $U$-action in the original construction of Ozsváth and Szabó.

Let us assume, once again, that $(\Sigma, \boldsymbol{\alpha}, \boldsymbol{\beta}, \mathbf{z})$ is an $\mathfrak{s}$-admissible Heegaard diagram for the sutured manifold $(X, \tau)$, where $\mathfrak{s} \in \operatorname{Spin}^{c}(\bar{X})$ is a fixed $\operatorname{Spin}^{c}$ structure. Let $\Omega\left(\mathbb{T}_{\alpha}, \mathbb{T}_{\beta}\right)$ denote the space of paths connecting $\mathbb{T}_{\alpha}$ to $\mathbb{T}_{\beta}$ in $\operatorname{Sym}^{\ell}(\Sigma)$. Any intersection point $\mathbf{x} \in \mathbb{T}_{\alpha} \cap \mathbb{T}_{\beta}$, viewed as a constant path, is a point in $\Omega\left(\mathbb{T}_{\alpha}, \mathbb{T}_{\beta}\right)$, and for any $\mathbf{x}, \mathbf{y} \in \mathbb{T}_{\alpha} \cap \mathbb{T}_{\beta}$ and any Whitney disk $u$ representing a class $\phi \in \pi_{2}(\mathbf{x}, \mathbf{y})$, $u$ may be viewed as a path connecting $\mathbf{x}$ to $\mathbf{y}$ in $\Omega\left(\mathbb{T}_{\alpha}, \mathbb{T}_{\beta}\right)$. The homotopy class of this path depends only on $\phi$. As in section 4 of OS5 for any one-cocycle

$$
\zeta \in Z^{1}\left(\Omega\left(\mathbb{T}_{\alpha}, \mathbb{T}_{\beta}\right), \mathbb{Z}\right)
$$

the evaluation $\zeta(\phi)$ is well-defined. Correspondingly, we may define the map

$$
\begin{aligned}
A_{\zeta} & : \mathrm{CF}(X, \tau ; \mathfrak{s}) \longrightarrow \mathrm{CF}(X, \tau ; \mathfrak{s}) \\
A_{\zeta}(\mathbf{x}) & :=\sum_{\substack{\mathbf{y} \in \mathbb{T}_{\alpha} \cap \mathbb{T}_{\beta} \\
\mathfrak{s}(\mathbf{y}) \in \mathfrak{s}}} \sum_{\substack{\phi \in \pi_{2}^{+}(\mathbf{x}, \mathbf{y}) \\
\mu(\phi)=1}}\left(\zeta(\phi) \cdot \lambda_{\mathbf{z}}(\phi) \cdot \mathfrak{m}(\phi)\right) \cdot \mathbf{y}, \quad \forall \mathbf{x} \in \mathbb{T}_{\alpha} \cap \mathbb{T}_{\beta}, \quad \text { s.t. } \mathfrak{s}(\mathbf{x}) \in \mathfrak{s} .
\end{aligned}
$$


The map $A_{\zeta}$ is then extended as a homomorphism of $\mathbb{A}$-modules to $\operatorname{CF}(X, \tau ; \mathfrak{s})$. It respects the decomposition according to relative $\operatorname{Spin}^{c}$ structures in $\mathfrak{s} \subset \operatorname{Spin}^{c}(X, \tau)$. As in lemmas 4.18 and 4.19 from OS5] one may prove that the map $A_{\zeta}$ satisfies

(i) $\quad \partial \circ A_{\zeta}+A_{\zeta} \circ \partial=0, \quad \&$

(ii) $A_{\zeta}=\partial \circ H_{\zeta}-H_{\zeta} \circ \partial$, if $\zeta$ is a coboundary,

for some $\mathbb{A}$-module homomorphism $H_{\zeta}$ which respects the filtration in $\mathbb{H}$. As a result, the following proposition may be proved in this generalized setup.

Proposition 5.11. There is a natural action of $\mathrm{H}^{1}\left(\Omega^{1}\left(\mathbb{T}_{\alpha}, \mathbb{T}_{\beta}\right) ; \mathbb{Z}\right)$ on $\mathrm{CF}(X, \tau ; \mathfrak{s})$ lowering degree by one, which is well-defined up to filtered chain homotopy equivalence. Furthermore, this induces an action of the exterior algebra

$$
\wedge^{*}\left(\mathrm{H}_{1}(\bar{X} ; \mathbb{Z}) / \text { Tors }\right) \subset \wedge^{*}\left(\mathrm{H}^{1}\left(\Omega\left(\mathbb{T}_{\alpha}, \mathbb{T}_{\beta}\right), \mathbb{Z}\right)\right)
$$

on the module $\mathrm{CF}(X, \tau ; \mathfrak{s})$, which is well-defined up to chain homotopy equivalence.

Proof. One should simply repeat the proof of proposition 4.17 from OS5. From the properties stated in equation 9 and the isomorphisms

$\mathrm{H}^{1}\left(\Omega\left(\mathbb{T}_{\alpha}, \mathbb{T}_{\beta}\right) ; \mathbb{Z}\right) \cong \operatorname{Hom}\left(\pi_{1}\left(\Omega\left(\mathbb{T}_{\alpha}, \mathbb{T}_{\beta}\right)\right), \mathbb{Z}\right) \cong \pi_{2}\left(\operatorname{Sym}^{\ell}(\Sigma)\right) \oplus \operatorname{Hom}\left(\mathrm{H}^{1}(\bar{X}, \mathbb{Z}), \mathbb{Z}\right)$,

the proof of the above proposition is reduced to showing $A_{\zeta} \circ A_{\zeta}=0$. For this purpose, let $f: \Omega\left(\mathbb{T}_{\alpha}, \mathbb{T}_{\beta}\right) \rightarrow S^{1}$ denote a representative of $\zeta$. For a generic point $p \in S^{1}$ we set $V_{p}=f^{-1}(p)$ and observe that for any generator $\mathbf{x} \in \mathbb{T}_{\alpha} \cap \mathbb{T}_{\beta}$ representing the $\operatorname{Spin}^{c}$ class $\mathfrak{s}$

$$
\begin{aligned}
A_{\zeta}(\mathbf{x}) & =\sum_{\substack{\mathbf{y} \in \mathbb{T}_{\alpha} \cap \mathbb{T}_{\beta} \\
\underline{\mathfrak{s}}(\mathbf{y}) \in \mathfrak{s}}} \sum_{\substack{\boldsymbol{c}\left(\pi_{2}^{+}(\mathbf{x}, \mathbf{y}) \\
\mu(\phi)=1\right.}}\left(a(\zeta, \phi) \cdot \lambda_{\mathbf{z}}(\phi)\right) \cdot \mathbf{y}, \\
\text { where } \quad a(\zeta, \phi) & =\#\left\{u \in \mathcal{M}(\phi) \mid u([0,1] \times\{0\}) \in V_{p}\right\} .
\end{aligned}
$$

Let us now consider a positive homotopy class $\phi \in \pi_{2}^{+}(\mathbf{x}, \mathbf{y})$ with $\mu(\phi)=2$. Associated with $\phi$, and for generic points $p, q \in S^{1}$, we consider the one-dimensional moduli space

$$
\Xi_{p, q}(\phi):=\left\{(s, u) \in[0, \infty) \times \mathcal{M}(\phi) \mid \begin{array}{c}
u([0,1] \times\{s\}) \subset V_{p} \\
u([0,1] \times\{-s\}) \subset V_{q}
\end{array}\right\} .
$$

This one-manifold does not have any boundary at $s=0$. Furthermore, if we set $I_{0}=[0,1] \times\{0\}$, the boundary at infinity (i.e. the structure of the moduli space as $s \rightarrow \infty)$ is modeled on

$$
\coprod_{\substack{\phi_{1} \times \phi_{2}=\phi \\ \mu\left(\phi_{1}\right)=\mu\left(\phi_{2}\right)=1}}\left(\left\{u_{1} \in \mathcal{M}\left(\phi_{1}\right) \mid u_{1}\left(I_{0}\right) \subset V_{p}\right\} \times\left\{u_{2} \in \mathcal{M}\left(\phi_{2}\right) \mid u_{2}\left(I_{0}\right) \subset V_{q}\right\}\right) .
$$

Other possible boundary points correspond to boundary disk degenerations and sphere bubblings. If we furthermore assume that $\lambda_{\mathbf{z}}(\phi) \neq 0$, any boundary disk degeneration or sphere bubbling will reduce the Maslov index at least by 2 . Thus the moduli space corresponding to such degenerations would be empty, if we choose a generic path of almost complex structures. 
The number of points in the boundary of $\Xi_{p, q}(\phi)$, counted with sign, would vanish. On the other hand, this total count corresponds to the contribution of the pairs $\left(\phi_{1}, \phi_{2}\right)$ with $\phi=\phi_{1} \star \phi_{2}$ and $\mu\left(\phi_{1}\right)=\mu\left(\phi_{2}\right)=1$ to the coefficient of $\lambda_{\mathbf{z}}(\phi) . \mathbf{y}$ in $A_{\zeta}^{2}(\mathbf{x})$. Thus $A_{\zeta}^{2}=0$ for all $\zeta \in \mathrm{H}^{1}\left(\Omega\left(\mathbb{T}_{\alpha}, \mathbb{T}_{\beta}\right), \mathbb{Z}\right)$. Thus the action descends to an action of the exterior algebra

$$
\wedge^{*}\left(\mathrm{H}^{1}\left(\Omega\left(\mathbb{T}_{\alpha}, \mathbb{T}_{\beta}\right), \mathbb{Z}\right)\right)
$$

This completes the proof of the proposition. 


\section{INVARIANCE OF THE FILTERED CHAIN HOMOTOPY TYPE}

6.1. Pseudo-holomorphic $m$-gons. Let us assume that the Heegaard diagram $H=\left(\Sigma, \boldsymbol{\alpha}^{1}, \boldsymbol{\alpha}^{2}, \ldots, \boldsymbol{\alpha}^{m}, \mathbf{z}\right)$ is given, so that $\Sigma$ is a closed Riemann surface of genus $g$, each $\boldsymbol{\alpha}^{i}$ is a set of $\ell$ disjoint simple closed curves on $\Sigma$, and $\mathbf{z}=\left\{z_{1}, \ldots, z_{\kappa}\right\}$ is a set of marked points in

$$
\Sigma-\boldsymbol{\alpha}^{1}-\boldsymbol{\alpha}^{2}-\ldots-\boldsymbol{\alpha}^{m}
$$

We assume that for all $i=1, . ., m$, every connected component in $\Sigma-\boldsymbol{\alpha}^{i}$ contains at least one element of $\mathbf{z}$. The Heegaard diagram $\left(\Sigma, \boldsymbol{\alpha}^{i}, \boldsymbol{\alpha}^{j}, \mathbf{z}\right)$ determines a balanced sutured manifold $\left(X_{i j}, \tau_{i j}\right)$. Let $\overline{X_{i j}}$ denote the three-manifold obtained from $X_{i j}$ by filling out the sutures in $\tau_{i j}$, and fix the $\operatorname{Spin}^{c}$ classes $\mathfrak{s}_{i j} \in \operatorname{Spin}^{c}\left(\overline{X_{i j}}\right)$. Assume that for any pair of indices $i<j,\left(\Sigma, \boldsymbol{\alpha}^{i}, \boldsymbol{\alpha}^{j}, \mathbf{z}\right)$ is an $\mathfrak{s}_{i j}$-admissible Heegaard diagram for the balanced sutured manifold $\left(X_{i j}, \tau_{i j}\right)$. Furthermore, let $\mathfrak{o}_{i j}$ be a coherent system of orientations on $\left(\Sigma, \boldsymbol{\alpha}^{i}, \boldsymbol{\alpha}^{j}, \mathbf{z}\right)$ associated with $\mathfrak{s}_{i j}$. Finally, suppose that

$$
\mathrm{CF}\left(\Sigma, \boldsymbol{\alpha}^{i}, \boldsymbol{\alpha}^{j}, \mathbf{z} ; \mathfrak{s}_{i j}\right)=\bigoplus_{\mathfrak{s}_{i j} \in \mathfrak{s}_{i j}} \mathrm{CF}\left(\Sigma, \boldsymbol{\alpha}^{i}, \boldsymbol{\alpha}^{j}, \mathbf{z} ; \mathfrak{\mathfrak { s }}_{i j}\right)
$$

is the corresponding chain complex, and its decomposition into relative $\operatorname{Spin}^{c}$ classes. Let us assume that

$$
\Sigma-\boldsymbol{\alpha}^{i}=\coprod_{j=1}^{k_{i}} A_{j}^{i}, \quad i=1,2, \ldots, m
$$

are the connected components in the complements of the curves in $\boldsymbol{\alpha}^{i}$. We will denote the genus of $A_{j}^{i}$ by $g_{j}^{i} \in \mathbb{Z}^{\geq 0}$. We will also denote

$$
\sum_{p=1}^{k_{i}} \lambda\left(A_{p}^{i}\right) \in\left\langle\lambda_{1}, \ldots, \lambda_{\kappa}\right\rangle_{\mathbb{Z}}
$$

by $\lambda\left(\boldsymbol{\alpha}^{i}\right)$. For any subset $I$ of the set of indices $\{1, \ldots, m\}$ introduce the $\mathbb{Z}$-algebra

$$
\mathbb{A}_{I}=\frac{\left\langle\lambda_{1}, \ldots, \lambda_{\kappa}\right\rangle_{\mathbb{Z}}}{\left\langle\lambda\left(\boldsymbol{\alpha}^{i}\right)=\lambda\left(\boldsymbol{\alpha}^{j}\right) \mid \forall i, j \in I\right\rangle \oplus\left\langle\lambda\left(A_{j}^{i}\right) \mid i \in I, g_{j}^{i}>0\right\rangle} .
$$

If for two subsets $I, J \subset\{1, \ldots, m\}$ we have $I \subset J$, then $\mathbb{A}_{J}$ would be a quotient of $\mathbb{A}_{I}$, and we have a natural homomorphism

$$
\rho_{I J}: \mathbb{A}_{I} \longrightarrow \mathbb{A}_{J} \text {. }
$$

This homomorphism may be used to give $\mathbb{A}_{J}$ the structure of an $\mathbb{A}_{I}$-module. As a result, from any $\mathbb{A}_{I}$ chain complex $(C, d)$, we obtain a natural $\mathbb{A}_{J}$ chain complex $C \otimes_{\mathbb{A}_{I}} \mathbb{A}_{J}$. In particular, for any index set $I$ which contains $i, j$, we may consider the $\mathbb{A}_{I}$ chain complex

$$
C_{i j}(I)=\mathrm{CF}\left(\Sigma, \boldsymbol{\alpha}^{i}, \boldsymbol{\alpha}^{j}, \mathbf{z} ; \mathfrak{s}_{i j}\right) \otimes_{\mathbb{A}_{i j}} \mathbb{A}_{I} .
$$

We will denote $C_{i j}(\{1, \ldots, m\})$ by $C_{i j}$ for simplicity.

Associated with each set of curves $\boldsymbol{\alpha}^{i}$ is a torus $\mathbb{T}_{\alpha^{i}} \subset \operatorname{Sym}^{\ell}(\Sigma)$. A Whitney $m$-gon is a continuous map $u$ from the standard $m$-gon $\mathbb{D}_{m}$ into $\operatorname{Sym}^{\ell}(\Sigma)$ which maps the $i$-th edge of the $m$-gon to $\mathbb{T}_{\alpha^{i}}$. If we fix

$$
\mathbf{x}_{i} \in \mathbb{T}_{\alpha^{i}} \cap \mathbb{T}_{\alpha^{i+1}}, \quad i=1, \ldots, m-1, \quad \& \quad \mathbf{x}_{m} \in \mathbb{T}_{\alpha^{m}} \cap \mathbb{T}_{\alpha^{1}},
$$


we may let $\pi_{2}\left(\mathbf{x}_{1}, \ldots, \mathbf{x}_{m}\right)$ denote the set of Whitney $m$-gons which map the vertex $v_{i}$ between the $i$-th edge and the $(i+1)$-th edge to $\mathbf{x}_{i}$ (for $\left.i=1, \ldots, m-1\right)$, and the vertex $v_{m}$ between the $m$-th edge and the first edge to $\mathbf{x}_{m}$.

Let us fix a generic continuous family $\left\{J_{p}\right\}_{p \in \mathbb{D}_{m}}$ of almost complex structures on $\operatorname{Sym}^{\ell}(\Sigma)$ determined by a family $\left\{j_{p}\right\}_{p \in \mathbb{D}_{m}}$ of complex structures on $\Sigma$. Furthermore, we will assume that under a fixed identification of a neighborhood of the $i$-th vertex $v_{i}$ of $\mathbb{D}_{m}$ with $[0,1] \times(0, \infty)$ the family is translation invariant, i.e.

$$
j_{(s, t)}=j_{(s, t+R)}, \quad \forall(s, t) \in[0,1] \times(0, \infty), \quad R \in \mathbb{R}^{+} .
$$

We will drop this generic family $\left\{J_{p}\right\}_{p \in \mathbb{D}_{m}}$ from our notation. For $\phi \in \pi_{2}\left(\mathbf{x}_{1}, \ldots, \mathbf{x}_{m}\right)$ we let $\mathcal{M}(\phi)$ denote the set of pseudo-holomorphic representatives of $\phi$.

Fix a subset $I=\left\{i_{1}<i_{2}<\ldots<i_{p}\right\} \subset\{1, \ldots, m\}$. This subset determines a sub-diagram

$$
H_{I}=\left(\Sigma, \boldsymbol{\alpha}^{i_{1}}, \ldots, \boldsymbol{\alpha}^{i_{p}}, \mathbf{z}\right)
$$

of $H$. Correspondingly, we may consider the $p$-gons associated with $H_{I}$. We will say that two $p$-gons $\phi \in \pi_{2}\left(\mathbf{x}_{i_{1}}, \ldots, \mathbf{x}_{i_{p}}\right)$ and $\phi^{\prime} \in \pi_{2}\left(\mathbf{y}_{i_{1}}, \ldots, \mathbf{y}_{i_{p}}\right)$ are equivalent if and only if there exists Whitney disk classes $\psi_{i_{j}} \in \pi_{2}\left(\mathbf{x}_{i_{j}}, \mathbf{y}_{i_{j}}\right)$ for $j=1, \ldots, p$ such that $\phi$ is obtained from $\phi^{\prime}$ by juxtaposition of the disk $\psi_{i_{j}}$ at the vertices $\mathbf{y}_{i_{j}}$ for $j=1, \ldots, p$. The set of equivalence classes of such $p$-gons will be denoted by $\operatorname{Spin}^{c}(H, I)$. It is important to note that $\operatorname{Spin}^{c}(H,\{i, j\})$ determines a subset of the set of $\operatorname{Spin}^{c}$ structures on the three-manifold $\overline{X_{i j}}$, which are realized by the Heegaard diagram.

Definition 6.1. Suppose that we have a pair of index sets $I, J \subset\{1, \ldots, m\}$ such that $I=\left\{i_{1}<i_{2}<\ldots<i_{p}\right\}$ and $J=\left\{i_{r}=j_{1}<j_{2}<\ldots<j_{q}=i_{r+1}\right\}$. We will call the pair $I, J$ attachable, and define

$$
I \star J:=\left\{i_{1}<\ldots<i_{r}=j_{1}<j_{2}<\ldots<j_{q}=i_{r+1}<i_{r+2}<\ldots<i_{p}\right\} .
$$

We will denote $r$ by $r(I, J)$ for future reference. Suppose that $I$ and $J$ are attachable index sets as above, and that we are given a p-gon $\phi$ and a $q$-gon $\phi^{\prime}$

$$
\phi \in \pi_{2}\left(\mathbf{x}_{i_{1}}, \ldots, \mathbf{x}_{i_{p}}\right), \quad \& \quad \psi \in \pi_{2}\left(\mathbf{y}_{j_{1}}, \ldots, \mathbf{y}_{j_{p}}\right),
$$

where $\mathbf{x}_{i_{s}} \in \mathbb{T}_{\alpha^{i_{s}}} \cap \mathbb{T}_{\alpha^{i_{s}+1}}$ and $\mathbf{y}_{j_{s}} \in \mathbb{T}_{\alpha^{j_{s}}} \cap \mathbb{T}_{\alpha^{j_{s+1}}}$. Furthermore, assume that $\mathbf{x}_{i_{r}}=\mathbf{y}_{j_{q}}$. Then we may juxtapose $\phi$ and $\psi$ to obtain the class of some $(p+q-2)$ gon, which will be denoted by $\phi \star \psi$.

Let us now restrict ourselves to the polygons whose vertices correspond to the fixed set $\mathfrak{S}=\left\{\mathfrak{s}_{i j}\right\}_{i<j}$ of $\operatorname{Spin}^{c}$ structures. Let us denote the subset of $\operatorname{Spin}^{c}(H, I)$ which consists of polygons such that the $\operatorname{Spin}^{c}$ structures associated with the vertices are in $\mathfrak{S}$ by $\operatorname{Sppin}^{c}(H, I ; \mathfrak{S})$. Then, the above construction gives a well-defined operation between the equivalence classes of polygons in $\operatorname{Spin}^{c}(H, I ; \mathfrak{S})$. More precisely, if $I$ and $J$ are a pair of attachable index sets, we will have a map

$$
(. \star .): \operatorname{Spin}^{c}(H, I ; \mathfrak{S}) \times \operatorname{Spin}^{c}(H, J ; \mathfrak{S}) \longrightarrow \operatorname{Spin}^{c}(H, I \star J ; \mathfrak{S}),
$$

defined by the above juxtaposition process. 
Definition 6.2. With the above notation fixed, a coherent system of Spin ${ }^{c}$ structures on polygons for the Heegaard diagram $H=\left(\Sigma, \boldsymbol{\alpha}^{1}, \ldots, \boldsymbol{\alpha}^{m}, \mathbf{z}\right)$, and compatible with $\mathfrak{S}$ is a choice of classes

$$
\mathfrak{T}=\left\{\phi_{I} \in \operatorname{Spin}^{c}(H, I ; \mathfrak{S})|I \subset\{1, \ldots, m\},| I \mid \geq 3\right\}
$$

such that the following is satisfied. If $I$ and $J$ are attachable index sets, then we have

$$
\phi_{I \star J}=\phi_{I} \star \phi_{J} .
$$

Lemma 6.3. Let us assume that a coherent system $\left\{\phi_{I}\right\}_{I}$ of $\operatorname{Spin}^{c}$ structures is fixed for the Heegaard diagram $H$. If $K=I \star J$ and a polygon $\psi_{K}$ in the same class as

$$
\phi_{K}=\phi_{I} \star \phi_{J} \in \operatorname{Spin}^{c}(H, K ; \mathfrak{S})
$$

is decomposed as $\psi_{K}=\psi_{I} \star \psi_{J}$, then the the class of $\psi_{I}$ in $\operatorname{Spin}^{c}(H, I ; \mathfrak{S})$ is equal to the class of $\phi_{I}$ and the class of $\psi_{J}$ in $\operatorname{Spin}^{c}(H, J ; \mathfrak{S})$ is equal to the class of $\phi_{J}$.

Proof. Fix the above notation and let $K=I \star J$. After addition of disk classes we may assume that the corners of $\psi_{K}$ are the same as the corners of $\phi_{K}$ (i.e. both are chosen from $\left\{\mathbf{x}_{i j}\right\}_{i<j}$ ). This means that

$$
\begin{aligned}
& \phi_{I} \in \pi_{2}\left(\mathbf{x}_{i_{1} i_{2}}, \ldots, \mathbf{x}_{i_{p-1} i_{p}}, \mathbf{x}_{i_{1} i_{p}}\right), \\
& \psi_{I} \in \pi_{2}\left(\mathbf{x}_{i_{1} i_{2}}, \ldots, \mathbf{x}_{i_{r-1} i_{r}}, \mathbf{y}, \mathbf{x}_{i_{r+1} i_{r+2}}, \ldots, \mathbf{x}_{i_{p-1} i_{p}}, \mathbf{x}_{i_{1} i_{p}}\right), \\
& \phi_{J} \in \pi_{2}\left(\mathbf{x}_{j_{1} j_{2}}, \ldots, \mathbf{x}_{j_{q-1} j_{q}}, \mathbf{x}_{j_{1} j_{q}}\right), \quad \& \quad \psi_{J} \in \pi_{2}\left(\mathbf{x}_{j_{1} j_{2}}, \ldots, \mathbf{x}_{j_{q-1} j_{q}}, \mathbf{y}\right)
\end{aligned}
$$

and we have $\phi_{I} \star \phi_{J}=\psi_{I} \star \psi_{J}$. We thus have the following relation among the associated domains:

$$
\mathcal{D}\left(\phi_{I}\right)-\mathcal{D}\left(\psi_{I}\right)=\mathcal{D}\left(\psi_{J}\right)-\mathcal{D}\left(\phi_{J}\right)=\mathcal{D} .
$$

The coefficients of the domains in the expression appearing on the left hand side of the above equality on both sides of any curve in $\boldsymbol{\alpha}^{i}$, with $i \notin I$, are equal. Similarly, the coefficients of the domains in the expression appearing as the middle term in the above equality on the two sides of any curve in $\boldsymbol{\alpha}^{j}$, with $j \notin J$, are equal. This implies that $\partial(\mathcal{D})$ is included in

$$
\coprod_{i \in I \cap J} \boldsymbol{\alpha}^{i}=\boldsymbol{\alpha}^{i_{r}} \coprod \boldsymbol{\alpha}^{i_{r+1}}
$$

Thus, $\mathcal{D}$ is the domain associated with a disk in $\pi_{2}\left(\mathbf{x}_{i_{r} i_{r+1}}, \mathbf{y}\right)$, and the $\operatorname{Spin}^{c}$ class of $\psi_{I}$ is the same as that of $\phi_{I}$. Similarly, the $\operatorname{Spin}^{c}$ class of $\psi_{J}$ is the same as that of $\phi_{J}$. This completes the proof of the lemma.

The above lemma implies that a coherent system of $\operatorname{Spin}^{c}$ structures on polygons for $H$ is completely determined by the choice of triangle classes

$$
\left\{\phi_{i j k} \in \operatorname{Spin}^{c}(H,\{i, j, k\} ; \mathfrak{S}) \mid 1 \leq i<j<k \leq m\right\},
$$

which satisfy the following compatibility relation

$$
\phi_{i k l} \star \phi_{i j k}=\phi_{i j l} \star \phi_{j k l} \quad \forall 1 \leq i<j<k<l \leq m .
$$

Furthermore, the above lemma implies that for $\phi_{i j k}, \phi_{i k l}$ and $\phi_{i j l}$ as above, there exists at most one class $\phi_{j k l}$ such that equation 10 is satisfied. This observation 
implies that a coherent system of $\operatorname{Spin}^{c}$ classes of polygons for the Heegaard diagram $H$ is determined by the family of triangle classes

$$
\left\{\phi_{1 i j} \mid 1<i<j \leq m\right\} .
$$

However, this family should have the property that for any triple $1<i<j<k \leq m$ of indices, there is a triangle class $\psi$ such that

$$
\phi_{1 j k} \star \phi_{1 i j}=\phi_{1 i k} \star \psi \text {. }
$$

If this is the case, we will write

$$
\mathfrak{T}=\left\{\phi_{I}\right\}_{I}=\left\langle\phi_{1 i j} \mid 1<i<j \leq m\right\rangle .
$$

Let us fix a system $\mathfrak{T}$ of compatible $\operatorname{Spin}^{c}$ structures for the Heegaard diagram $H$ as above, which is generated by the triangle classes $\phi_{1 i j}$. The set of periodic domains for polygons in $\mathfrak{T}$ is generated by periodic domains for each pair $\left(\boldsymbol{\alpha}^{i}, \boldsymbol{\alpha}^{j}\right)$. To be more precise, let us denote by $\mathfrak{P}_{i j}$ the set of periodic domains for the Heegaard diagram $\left(\Sigma, \boldsymbol{\alpha}^{i}, \boldsymbol{\alpha}^{j}\right)$. Then any periodic domain which appears as the difference of two $q$-gons with the same set of vertices

$$
\mathbf{y}_{j} \in \mathbb{T}_{\alpha^{i_{j}}} \cap \mathbb{T}_{\alpha^{i_{j+1}}}, \quad j=1, \ldots, q, i_{q+1}:=i_{1}, \& i_{1}<i_{2}<\ldots<i_{q},
$$

and representing the same $\operatorname{Spin}^{c}$ class may be written as a sum of periodic domains in $\mathfrak{P}_{i_{1} i_{2}}, \mathfrak{P}_{i_{2} i_{3}}, \ldots, \mathfrak{P}_{i_{q-1} i_{q}}$, and $\mathfrak{P}_{i_{1} i_{q}}$.

Definition 6.4. Let the Heegaard diagram $H=\left(\Sigma, \boldsymbol{\alpha}^{1}, \boldsymbol{\alpha}^{2}, \ldots, \boldsymbol{\alpha}^{m}, \mathbf{z}\right)$ and $\mathfrak{S}, \mathfrak{T}$, and $\mathfrak{P}_{i j}$ be as above. The Heegaard diagram $H$ is called $\mathfrak{S}$-admissible if for any index set $I=\left\{i_{1}<\ldots<i_{q}\right\}$, and any periodic domain

$$
\mathcal{P}=\mathcal{P}_{i_{1} i_{2}}+\mathcal{P}_{i_{2} i_{3}}+\ldots+\mathcal{P}_{i_{q-1} i_{q}}+\mathcal{P}_{i_{1} i_{q}}
$$

with $\mathcal{P}_{i j} \in \mathfrak{P}_{i j}$, the following is true. If

$$
\sum_{j=1}^{q}\left\langle c_{1}\left(\mathfrak{s}_{i_{j} i_{j+1}}\right), H\left(\mathcal{P}_{i_{j} i_{j+1}}\right)\right\rangle=0
$$

then either the coefficients of the domain $\mathcal{P}$ at some point $w$ is negative, or $\lambda(\mathcal{P})=0$ in $\mathbb{A}_{I}$.

The existence of $\mathfrak{S}$-admissible Heegaard diagrams, and the possibility of modifying $H$ to an admissible Heegaard diagram using finger moves, follows with an argument completely similar to the arguments of section 4 . Furthermore, the $\mathfrak{S}$ admissibility of the Heegaard diagram $H$ implies that for any index set

$$
I=\left\{i_{1}<\ldots<i_{q}\right\} \subset\{1, \ldots, m\},
$$

any integer $N$, and any set of corners

$$
\mathbf{y}_{j} \in \mathbb{T}_{\alpha^{i j}} \cap \mathbb{T}_{\alpha^{i j}+1}, \quad j=1, \ldots, q, i_{q+1}:=i_{1}, \& i_{1}<i_{2}<\ldots<i_{q},
$$

such that $\underline{\underline{\mathfrak{z}}}_{\mathbf{z}}\left(\mathbf{y}_{j}\right) \in \mathfrak{s}_{i_{j} i_{j+1}}$, there are at most finitely many classes $\phi \in \pi_{2}\left(\mathbf{y}_{1}, \ldots, \mathbf{y}_{q}\right)$ satisfying the following three conditions.

- $\phi=\phi_{I} \in \operatorname{Spin}^{c}(H, I ; \mathfrak{S})$.

- $\mu(\phi)=N$. 
- $\mathcal{D}(\phi) \geq 0$ and $\lambda_{\mathbf{z}}(\phi ; I) \neq 0$, where $\lambda_{\mathbf{z}}(\phi ; I)$ is defined by

$$
\lambda_{\mathbf{z}}(\phi ; I):=\prod_{i=1}^{\kappa} \lambda_{i}^{n_{z_{i}}(\phi)} \in \mathbb{A}_{I} .
$$

The construction of Ozsváth and Szabó in subsection 8.2 from OS5 may be extended to this more general context without any major modification. Namely, for any index set $I \subset\{1, \ldots, m\}$, and any polygon class $\phi \in \phi_{I}$, the determinant line bundle of the Cauchy-Riemann operator over $\mathcal{M}(\phi)$ is trivial, and one may thus choose an orientation, i.e. one of the two classes of nowhere vanishing sections of this determinant line bundle, associated with $\phi$.

Definition 6.5. A coherent system of orientations associated with the Heegaard diagram $H$ and the coherent system $\mathfrak{T}$ of $\operatorname{Spin}^{c}$ classes of polygons of $H$ is a choice of orientation $\mathfrak{o}_{I}(\phi)$ for any polygon class $\phi$ with $\phi=\phi_{I} \in \operatorname{Spin}^{c}(H, I ; \mathfrak{S})$, such that the following are satisfied.

- For any $1 \leq i<j \leq m, \mathfrak{o}_{i j}$ is a coherent system of orientations associated with the $\operatorname{Spin}^{c}$ class $\mathfrak{s}_{i j}$ for the Heegaard diagram $\left(\Sigma, \boldsymbol{\alpha}^{i}, \boldsymbol{\alpha}^{j}, \mathbf{z}\right)$.

- For any pair $I, J$ of attachable index sets and any attachable polygon classes $\phi$ and $\psi$, with

$$
\phi=\phi_{I} \in \operatorname{Spin}^{c}(H, I ; \mathfrak{S}), \quad \& \quad \psi \in \operatorname{Spin}^{c}(H, J ; \mathfrak{S}),
$$

we have

$$
(-1)^{r(I, J)|J|} \mathfrak{o}_{I}(\phi) \wedge \mathfrak{o}_{J}(\psi)=\mathfrak{o}_{I \star J}(\phi \star \psi) .
$$

Lemma 6.3 implies that in order for us to obtain a coherent system of orientations associated with the Heegaard diagram $H$ and the coherent system of $\operatorname{Spin}^{c}$ classes of polygons $\mathfrak{T}$, it suffices to determine $\mathfrak{o}_{i j}$ and $\mathfrak{o}_{1 i j}$ for any pair of indices $1 \leq i<j \leq m$. This observation implies that the following lemma, which was proved in OS5 as lemma 8.7, is valid in our setup. Although the Heegaard diagrams are more general, the proof carries over without any major modification.

Lemma 6.6. Suppose that the Heegaard diagram H, and the coherent system of Spin $^{c}$ classes of polygons $\mathfrak{T}$ are as above. Then for any choice of coherent systems of orientations $\mathfrak{o}_{1 i}$ corresponding to the $\operatorname{Spin}^{c}$ classes $\mathfrak{s}_{1 i}$ (with $1<i \leq m$ ), and any choice of $\mathfrak{o}_{1 i j}\left(\phi_{1 i j}\right)$ for $1<i<j \leq m$, there always exists a coherent system of orientations $\mathfrak{o}=\left\{\mathfrak{o}_{I}\right\}_{I}$ such that $\mathfrak{o}_{1 i}$ is the initial choice of the coherent system of orientations corresponding to $\mathfrak{s}_{1 i}$ and $\mathfrak{o}_{1 i j}\left(\phi_{1 i j}\right)$ is the prescribed orientation.

Proof. For an index set $I=\left\{i_{1}<\ldots<i_{q}\right\}$ let $i(I)=i_{1}$ and $j(I)=i_{q}$ denote the smallest and largest element of $I$ respectively. Let us assume that $\phi$ is a $q$-gon class in the same $\operatorname{Spin}^{c}$ class as $\phi_{I}$.

If $1 \in I$, then we may assume $|I| \geq 3$, since otherwise, we already have a choice of orientation. In this case, we may write, in a unique way,

$$
\begin{aligned}
& \phi=\phi_{I} \star \phi_{1} \star \ldots \star \phi_{q}, \quad \phi_{j} \in \pi_{2}\left(\mathbf{y}_{j}, \mathbf{x}_{i_{j} i_{j+1}}\right) \\
& \phi_{I}=\phi_{1 i_{2} i_{3}} \star \phi_{1 i_{3} i_{4}} \star \ldots \star \phi_{1 i_{q-1} i_{q}} .
\end{aligned}
$$

Thus $\mathfrak{o}_{I}(\phi)$ is determined if we determine all the maps $\mathfrak{o}_{i j}$ for $1<i<j \leq m$ in a compatible way. Note that $\mathfrak{o}_{1 i j}\left(\phi_{1 i j}\right)$ is already defined. If otherwise $1 \notin I$, we may 
write, again in a unique way

$$
\begin{aligned}
& \phi=\phi_{I} \star \phi_{1} \star \ldots \star \phi_{q}, \quad \phi_{j} \in \pi_{2}\left(\mathbf{y}_{j}, \mathbf{x}_{i_{j} i_{j+1}}\right) \\
& \phi_{1 i(I) j(I)} \star \phi_{I}=\phi_{1 i_{1} i_{2}} \star \phi_{1 i_{2} i_{3}} \star \ldots \star \phi_{1 i_{q-1} i_{q}} .
\end{aligned}
$$

Thus, in order to determine the orientation $\mathfrak{o}_{I}(\phi)$, it suffices to determine all maps $\mathfrak{o}_{i j}$ for $1<i<j \leq m$. In order to determine the $\mathfrak{o}_{i j}$ from $\mathfrak{o}_{1 i}, \mathfrak{o}_{1 j}$ and $\mathfrak{o}_{1 i j}\left(\phi_{1 i j}\right)$, one may then use the argument of lemma 8.7 from [OS5].

Remark 6.7. Note that the choice of $\mathfrak{o}_{1 i}$ for $1<i \leq m$ determines the orientation for all boundary degenerations in a unique way. In fact, suppose that $\psi$ is the class of some $\boldsymbol{\alpha}^{i}$ boundary degeneration corresponding to the corner $\mathbf{y} \in \mathbb{T}_{\alpha^{i}} \cap \mathbb{T}_{\alpha^{j}}$, say for some $j>i$, and that $\phi$ is a Whitney disk in $\pi_{2}\left(\mathbf{x}_{i j}, \mathbf{y}\right)$. Furthermore, let $\psi^{\prime}$ denote the class in $\pi_{2}^{\alpha^{i}}\left(\mathbf{x}_{1 i}\right)$ which has the same domain as $\psi$. We may then write

$$
\phi_{1 i j} \star \phi \star \psi=\phi_{1 i j} \star \psi^{\prime} \star \phi,
$$

implying that $\mathfrak{o}_{i j}(\psi)$ is uniquely determined by $\mathfrak{o}_{1 i}\left(\psi^{\prime}\right)$, and is equal to it as the class of an $\alpha^{i}$ boundary degeneration.

Let $H$ be an $\mathfrak{S}$-admissible Heegaard diagram, and $\mathfrak{T}$ be a system of compatible $\operatorname{Spin}^{c}$ structures as before. Correspondingly, assume that

$$
\mathfrak{o}=\left\{\mathfrak{o}_{I}|I \subset\{1, \ldots, m\},| I \mid \geq 2\right\}
$$

is a coherent system of orientations associated with $\mathfrak{T}$. Associated with any subset $I=\left\{i_{1}, \ldots, i_{q}\right\} \subset\{1, \ldots, m\}$ of indices, we may define a holomorphic polygon map

$$
\begin{aligned}
f_{I}: \bigotimes_{j=1}^{q-1} \operatorname{CF}\left(\Sigma, \boldsymbol{\alpha}^{i_{j}}, \boldsymbol{\alpha}^{i_{j+1}}, \mathbf{z} ; \mathfrak{s}_{i_{j} i_{j+1}}\right) \otimes_{\mathbb{A}_{\left\{i_{j}, i_{j+1}\right\}}} \mathbb{A}_{I} \\
\longrightarrow \operatorname{CF}\left(\Sigma, \boldsymbol{\alpha}^{i_{1}}, \boldsymbol{\alpha}^{i_{q}}, \mathbf{z} ; \mathfrak{s}_{i_{1} i_{q}}\right) \otimes_{\mathbb{A}_{\left\{i_{1}, i_{q}\right\}}} \mathbb{A}_{I} .
\end{aligned}
$$

In other words, if $\{i<j\} \triangleleft I$ denotes that $i$ and $j$ are consecutive elements in $I$, and $i(I), j(I)$ denote the smallest and largest elements of $I$ respectively, we will have a map

$$
\begin{aligned}
& f_{I}: \bigotimes_{\{i<j\} \triangleleft I} C_{i j}(I)=\bigotimes_{j=1}^{q-1} C_{i_{j} i_{j+1}}(I) \longrightarrow C_{i_{1} i_{q}}(I)=C_{i(I), j(I)}(I) \\
& f_{I}\left(\mathbf{y}_{1} \otimes \mathbf{y}_{2} \otimes \ldots \otimes \mathbf{y}_{q-1}\right):=\sum_{\substack{\mathbf{y}_{q} \in \mathbb{T}_{\beta} \cap \mathbb{T}_{\beta} q \\
\left[\mathfrak{s}\left(\mathbf{y}_{q}\right)\right]=\mathfrak{t}_{1 q}}} \sum_{\substack{\phi \in \pi_{2}\left(\mathbf{y}_{1}, \mathbf{y}_{2}, \ldots, \mathbf{y}_{q}\right) \\
\mu(\phi)=3-q \\
\phi \in\left(\phi_{I}\right)}}\left(\mathfrak{m}(\phi) \lambda_{\mathbf{z}}(\phi ; I)\right) \cdot \mathbf{y}_{q},
\end{aligned}
$$

where $\boldsymbol{\beta}^{j}=\boldsymbol{\alpha}^{i_{j}}$ and $\mathfrak{t}_{1 q}=\mathfrak{s}_{i_{1} i_{q}}$.

Since $H$ is admissible, it follows that only finitely many terms would contribute to the above sum, and $f_{I}$ is thus well-defined.

These maps satisfy a generalized associativity property, which may be stated in our setup as follows (we will only state the associativity corresponding to the full index set $\{1, \ldots, m\})$. 
Theorem 6.8. With the above notation fixed, if we set $[m]=\{1, \ldots, m\}$ as the full index set, the map

$$
\begin{aligned}
& F_{[m]}: C_{12}([m]) \otimes C_{23}([m]) \otimes \ldots \otimes C_{m-1, m}([m]) \longrightarrow C_{1 m}([m]), \\
& F_{[m]}:=\sum_{1 \leq i<j \leq m}(-1)^{i j} f_{\{1,2, \ldots, i, j, j+1, \ldots, m\}} \circ f_{\{i, i+1, \ldots, j\}}
\end{aligned}
$$

is trivial.

Proof. Let us denote the set $\{1,2, \ldots, i, j, j+1, \ldots, m\}$ of indices by $I(i, j)$, and $\{i, i+1, \ldots, j\}$ by $J(i, j)$. We have to show that for any set $\mathbf{y}_{1}, \ldots, \mathbf{y}_{m}$ of intersection points with $\mathbf{y}_{i} \in \mathbb{T}_{\alpha^{i}} \cap \mathbb{T}_{\alpha^{i+1}}$, and such that $\underline{\mathfrak{s}}\left(\mathbf{y}_{i}\right) \in \mathfrak{s}_{i,(i+1)}$, the coefficient of $\mathbf{y}_{m}$ in

$$
\sum_{1 \leq i<j \leq m}(-1)^{i j} f_{I(i, j)}\left(\mathbf{y}_{1} \otimes \ldots \otimes \mathbf{y}_{i-1} \otimes f_{J(i, j)}\left(\mathbf{y}_{i} \otimes \ldots \otimes \mathbf{y}_{j-1}\right) \otimes \mathbf{y}_{j} \otimes \ldots \otimes \mathbf{y}_{m-1}\right)
$$

is zero. Let us consider a Whitney polygon class $\psi \in \pi_{2}\left(\mathbf{y}_{1}, \ldots, \mathbf{y}_{m}\right)$ with Maslov index $4-m$ and in the same class as $\psi_{[m]}$, and consider the ends of $\mathcal{M}(\psi)$. The ends of this moduli space do not contain any boundary disk degenerations or sphere bubblings. The reason is that the Maslov index of the holomorphic boundary disk degenerations and holomorphic spheres are greater than or equal to 2 if the corresponding element of the coefficient ring is non-trivial. This would imply that the remaining component should have Maslov index at most $2-m$. As a result, the moduli space associated with the remaining part would be empty.

Thus all degenerations of this moduli space (for dimensional reasons) are degenerations along an arc which connects two different edges of the $m$-gon. The ends corresponding to a degeneration along an arc connecting the $i$-th edge to the $j$-th edge, with $i<j$ correspond to a degeneration of $\psi$ into the juxtaposition of a holomorphic Whitney $(j-i+1)$-gon connecting $\mathbf{y}_{i}, \ldots, \mathbf{y}_{j-1}$ to an intersection point $\mathbf{x} \in \mathbb{T}_{\alpha^{i}} \cap \mathbb{T}_{\alpha^{j}}$ with Maslov index $2-j+i$, with a holomorphic $(m-j+i+1)$-gon connecting $\mathbf{y}_{1}, \ldots, \mathbf{y}_{i-1}, \mathbf{x}, \mathbf{y}_{j}, \ldots \mathbf{y}_{m-1}$ to $\mathbf{y}_{m}$ with Maslov index $2-m+j-i$. Thus, the ends of $\mathcal{M}(\psi)$ will have the following form.

$$
\partial \mathcal{M}(\psi)=\coprod_{\substack{1 \leq i<j \leq m \\ \mathbf{x} \in \mathbb{T}_{\alpha^{i}} \cap \mathbb{T}_{\alpha^{j}}}} \coprod_{\substack{\phi_{i j} \in \pi_{2}^{2-j+i}\left(\mathbf{y}_{i}, \ldots, \mathbf{y}_{j-1}, \mathbf{x}\right) \\ \psi_{i j} \in \pi_{2}^{2-m+j-i}\left(\mathbf{y}_{1}, \ldots, \mathbf{y}_{i-1}, \mathbf{x}, \mathbf{y}_{j}, \ldots, \mathbf{y}_{m}\right) \\ \psi_{i j} \star \phi_{i j}=\psi}}\left(\mathcal{M}\left(\psi_{i j}\right) \times \mathcal{M}\left(\phi_{i j}\right)\right) .
$$

In the above decomposition, we are dropping the condition that the polygons represent the $\operatorname{Spin}^{c}$ class determined by $\mathfrak{T}$. The sign difference between the orientation we assign to the component $\mathcal{M}\left(\psi_{i j}\right) \times \mathcal{M}\left(\phi_{i j}\right)$, and its orientation as a boundary component of $\partial \mathcal{M}(\psi)$ is computed as

$$
\epsilon\left(\mathcal{M}\left(\psi_{i j}\right) \times \mathcal{M}\left(\phi_{i j}\right)\right)=(-1)^{r(I(i, j), J(i, j))|J(i, j)|}=(-1)^{i(j-i+1)}=(-1)^{i j} .
$$

Note that the total number of points in the moduli space on the right hand side of the above equation, when counted with the above induced signs, will be zero. We should of course mod out by possible automorphisms of the domain, when necessary. Fix a generator $\lambda \in G\left(\mathbb{A}_{[m]}\right)$. The coefficient of $\lambda . \mathbf{y}_{m}$ in

$$
\sum_{1 \leq i<j \leq m}(-1)^{i j}\left(\mathbf{y}_{1} \otimes \ldots \otimes \mathbf{y}_{i-1} \otimes f_{J(i, j)}\left(\mathbf{y}_{i} \otimes \ldots \otimes \mathbf{y}_{j-1}\right) \otimes \mathbf{y}_{j} \otimes \ldots \otimes \mathbf{y}_{m-1}\right)
$$


is equal to

$$
\begin{aligned}
& \sum_{\substack{1 \leq i<j \leq m \\
\mathbf{x} \in \mathbb{T}_{\alpha}{ }^{i} \cap \mathbb{T}_{\alpha j} j}} \sum_{\substack{\phi_{i j} \in \pi_{2}^{2-j+i}\left(\mathbf{y}_{i}, \ldots, \mathbf{y}_{j-1}, \mathbf{x}\right) \\
\psi_{i j} \in \pi_{2}^{2-m+j-i}\left(\mathbf{y}_{1}, \ldots, \mathbf{y}_{i-1}, \mathbf{x}, \mathbf{y}_{j}, \ldots, \mathbf{y}_{m}\right) \\
\lambda\left(\phi_{i j}\right) \lambda\left(\psi_{i j}\right)=\lambda}}(-1)^{i j}\left(\mathfrak{m}\left(\psi_{i j}\right) \mathfrak{m}\left(\phi_{i j}\right)\right) \\
& =\sum_{\substack{\psi \in \pi_{2}^{4-m}\left(\mathbf{y}_{1}, \ldots, \mathbf{y}_{m}\right) \\
\lambda(\psi)=\lambda}} \#(\partial(\mathcal{M}(\psi)))=0 .
\end{aligned}
$$

The above computation thus completes the proof of the theorem.

Remark 6.9. The maps

$$
f_{I}: \bigotimes_{\{i<j\} \triangleleft I} C_{i j}(I) \rightarrow C_{i(I) j(I)}(I)
$$

will sometimes refine to the maps respecting the relative $\operatorname{Spin}^{c}$ structures. We will face this situation in the upcoming sections several times. Each time we will give a separate argument for such an splitting, to avoid the complexity of a general treatment.

In order to prove the above associativity, we do not need to use the full system $\mathfrak{P}$ of compatible $\operatorname{Spin}^{c}$ structures. In fact, a subsystem containing the classes of polygons associated with the index sets $I(i, j)$ and $J(i, j)$ suffices for this purpose. In other words, we only make use of the $\operatorname{Spin}^{c}$ classes in the subset

$$
\mathfrak{T}_{1}=\left\{\phi_{I(i, j)} \mid 1 \leq i<j \leq m\right\} \cup\left\{\phi_{J(i, j)} \mid 1 \leq i<j \leq m\right\} \subset \mathfrak{T}
$$

for defining the maps appearing on the left-hand-side of equation 12

Definition 6.10. The set

$$
\begin{aligned}
\mathfrak{T}_{1}=\{ & \left.\phi_{I(i, j)} \in \operatorname{Spin}^{c}(H, I(i, j)) \mid 1 \leq i<j \leq m\right\} \\
& \bigcup\left\{\phi_{J(i, j)} \in \operatorname{Spin}^{c}(H, J(i, j)) \mid 1 \leq i<j \leq m\right\}
\end{aligned}
$$

of polygon classes is called a system of first degenerations for $\phi_{[m]} \in \operatorname{Spin}^{c}(H,[m] ; \mathfrak{S})$ if

$$
\phi_{I(i, j)} \star \phi_{J(i, j)}=\phi, \quad \forall 1 \leq i<j \leq m .
$$

Thus, instead of $\mathfrak{T}$, we may fix a system of first degenerations for a class $\phi_{[m]} \in \operatorname{Spin}^{c}(H,[m] ; \mathfrak{S})$, together with a compatible system of coherent orientations associated with them. Then theorem 6.8 would still remain true.

6.2. Special Heegaard diagrams corresponding to handle slides. Let us assume that $(\Sigma, \boldsymbol{\alpha}, \boldsymbol{\beta}, \mathbf{z})$ is a Heegaard diagram, which corresponds to a balanced sutured manifold $(X, \tau)$. Let us assume that

$$
\boldsymbol{\alpha}=\left\{\alpha_{1}, \ldots, \alpha_{\ell}\right\}, \quad \& \boldsymbol{\beta}=\left\{\beta_{1}, \ldots, \beta_{\ell}\right\}
$$

and that $\beta_{i}$ is the image of $\alpha_{i}$ under a small Hamiltonian isotopy for $i=2, \ldots, \ell$ so that $\beta_{i}$ is disjoint from $\alpha_{j}$ for $j \neq 1, i$ and cuts $\alpha_{i}$ in a pair of canceling intersection 
points. The area bounded between $\alpha_{i}$ and $\beta_{i}$ is thus of the form $\mathcal{P}_{i}=D_{i}^{+}-D_{i}^{-}$, such that $D_{i}^{+}$and $D_{i}^{-}$are two of connected components in

$$
\Sigma-\boldsymbol{\alpha}-\boldsymbol{\beta},
$$

and $\partial \mathcal{P}_{i}=\alpha_{i}-\beta_{i}$. Furthermore, assume that $\beta_{1}$ is obtained from $\alpha_{1}$ by first moving it by a small Hamiltonian isotopy, and then doing a handle slide along $\alpha_{2}$. Thus, the only curve in $\boldsymbol{\alpha} \cup \boldsymbol{\beta}$ that intersects $\beta_{1}$ is $\alpha_{1}$, which cuts $\beta_{1}$ in a pair of intersection points. These two intersection points are connected by a bi-gon, which we will denote by $D_{1}^{+}$. There is a domain with small area which is bounded between $\alpha_{1}, \beta_{1}, \alpha_{2}$ and $\beta_{2}$, denoted by $D_{1}^{-}$, so that $\mathcal{P}_{1}=D_{1}^{+}-D_{1}^{-}-D_{2}^{-}$is a periodic domain satisfying

$$
\partial \mathcal{P}_{1}=\alpha_{1}+\alpha_{2}-\beta_{1} .
$$

We will assume that none of the marked points $\mathbf{z}=\left\{z_{1}, . ., z_{\kappa}\right\}$ are in any of $D_{1}^{+}, \ldots, D_{\ell}^{+}$or $D_{1}^{-}, \ldots, D_{\ell}^{-}$. Let us assume

$$
\Sigma-\boldsymbol{\alpha}-\boldsymbol{\beta}=\left(\coprod_{i=1}^{\ell} D_{i}^{+}\right) \bigcup\left(\coprod_{i=1}^{\ell} D_{i}^{-}\right) \bigcup\left(\coprod_{i=1}^{m} E_{i}\right),
$$

and that $\mathbf{z}^{i}=\left\{z_{1}^{i}, \ldots, z_{j_{i}}^{i}\right\}$ are the marked points in $E_{i}$ for $i=1, \ldots, m$. Thus, $\mathbf{z}=\mathbf{z}^{1} \cup \ldots \cup \mathbf{z}^{m}$. In the ring $\mathbb{A}_{\tau}$, let $\lambda\left(z_{j}^{i}\right)$ denote the element associated with $z_{j}^{i} \in \mathbf{z}$. Furthermore, define

$$
\mu_{i}=\prod_{j=1}^{j_{i}} \lambda\left(z_{j}^{i}\right) \in \mathbb{A}_{\tau}, \quad i=1, \ldots, m .
$$

If $\Sigma-\boldsymbol{\alpha}=\coprod_{i=1}^{m_{a}} A_{i}$ and $\Sigma-\boldsymbol{\beta}=\coprod_{i=1}^{m_{b}} B_{i}$, we will have $m_{a}=m_{b}=m$, and after renaming the indices if necessary, we may assume $\mathbf{z} \cap A_{i}=\mathbf{z} \cap B_{i}$, and $\lambda\left(A_{i}\right)=\lambda\left(B_{i}\right)=\mu_{i}$. Let us denote by $\mathbb{A}_{\mu}$ the sub-ring of $\mathbb{A}_{\tau}$ generated by $\mu_{1}, \ldots, \mu_{m}$.

Any pair of curves $\left(\alpha_{i}, \beta_{i}\right)$ intersect in a pair $x_{i}^{+}, x_{i}^{-}$of points, so that the bi-gon $D_{i}^{+}$connects $x_{i}^{+}$to $x_{i}^{-}$. Any map $\epsilon:\{1, \ldots, \ell\} \rightarrow\{+,-\}$ thus corresponds to an intersection point

$$
\mathbf{x}^{\epsilon}=\left\{x_{1}^{\epsilon(1)}, x_{2}^{\epsilon(2)}, \ldots, x_{\ell}^{\epsilon(\ell)}\right\} \in \mathbb{T}_{\alpha} \cap \mathbb{T}_{\beta} .
$$

These all correspond to the same $\operatorname{Spin}^{c}$ class in $\operatorname{Spin}^{c}\left(\bar{X}^{\tau}\right)$, which will be denoted by $\mathfrak{s}_{0}$. For $\epsilon:\{1, \ldots, \ell\} \rightarrow\{+,-\}$ let $|\epsilon|$ denote the number of elements in $\epsilon^{-1}\{+\}$. We may refine the homological grading of the generators of $\mathrm{CF}\left(\Sigma, \boldsymbol{\alpha}, \boldsymbol{\beta}, \mathbf{z} ; \mathfrak{s}_{0}\right)$ into a relative $\mathbb{Z}$-grading by setting

$$
\operatorname{gr}(\epsilon, \delta)=|\epsilon|-|\delta|, \quad \forall \epsilon, \delta:\{1, \ldots, \ell\} \longrightarrow\{+,-\} .
$$

We will show below that this gives a well-defined relative grading in an appropriate sense.

The periodic domains corresponding to the above Heegaard diagram are generated, as a free abelian group, by $\mathcal{P}_{1}, \ldots, \mathcal{P}_{\ell}, A_{1}, \ldots, A_{m}$. Note that $\lambda\left(\mathcal{P}_{i}\right)=1$. If

$$
\mathcal{P}=q_{1} \mathcal{P}_{1}+\ldots+q_{\ell} \mathcal{P}_{\ell}+a_{1} A_{1}+\ldots+a_{m} A_{m} \geq 0
$$



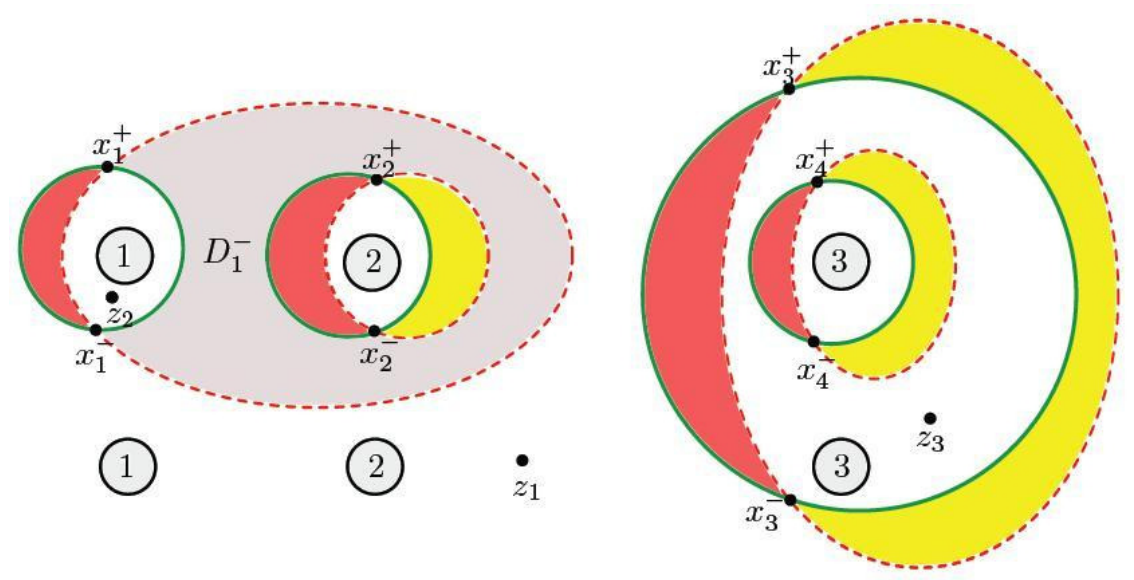

Figure 1. The green curves denote the elements of $\boldsymbol{\alpha}$ and the dashed red curves denote the elements of $\boldsymbol{\beta}$. The domains $D_{i}^{+}$are shaded red, while the domains $D_{i}^{-}$are shaded yellow. The domain $D_{1}^{-}$is shaded gray.

is a positive periodic domain with $\left\langle c_{1}(\mathfrak{s}), H(\mathcal{P})\right\rangle=0$ for some $\mathfrak{s} \in \operatorname{Spin}^{c}\left(\bar{X}^{\tau}\right)$, we will have $a_{1}, \ldots, a_{m} \geq 0$ and

$$
0=a_{1}\left(2-2 g\left(A_{1}\right)\right)+a_{2}\left(2-2 g\left(A_{2}\right)\right)+\ldots+a_{m}\left(2-2 g\left(A_{m}\right)\right) .
$$

Here $g\left(A_{i}\right)$ denotes the genus of $A_{i}$. The reason is that the evaluation of the $\operatorname{Spin}^{c}$ classes over the above periodic domains is independent of $\mathfrak{s}$. If moreover we know that $\lambda(\mathcal{P}) \neq 0$, we may conclude that if $a_{i} \neq 0$ then $g\left(A_{i}\right)=0$. Let us assume that $A_{1}, \ldots, A_{k}$ are the components of genus zero, and the rest of $A_{i}$ have positive genus. This implies that

$$
a_{1}, \ldots, a_{m} \geq 0, \quad 0=a_{1}+\ldots+a_{k}, \quad \& \quad a_{k+1}=\ldots=a_{m}=0 .
$$

Thus all $a_{i}$ are zero, and $\mathcal{P}=q_{1} \mathcal{P}_{1}+\ldots+q_{\ell} \mathcal{P}_{\ell}$. Since $\mathcal{P}_{2}, \ldots, \mathcal{P}_{\ell}$ are disjoint, and all $\mathcal{P}_{i}$ have both positive and negative coefficients, one can easily conclude that $\mathcal{P}$ has both positive and negative coefficients. Thus the constructed Heegaard diagram is admissible for all $\mathrm{Spin}^{c}$ classes.

One may choose an orientation $\mathfrak{o}$ for this Heegaard diagram. For any choice of orientation, one may observe that $D_{i}^{+}$and $D_{i}^{-}$are both domains of Whitney disks, for $i=2, \ldots, \ell$, and the number of points in

$$
\widehat{\mathcal{M}}\left(D_{i}^{+}\right) \bigcup \widehat{\mathcal{M}}\left(D_{i}^{-}\right)
$$

is zero (in fact, the orientation of the two moduli spaces are the opposite of one another, in some sense). Thus there is a filtered chain complex $\operatorname{CF}\left(\Sigma, \boldsymbol{\alpha}, \boldsymbol{\beta}, \mathbf{z} ; \mathfrak{s}_{0}\right)$ associated with the above Heegaard diagram, which is freely generated by the set $\left\{\mathbf{x}^{\epsilon}\right\}_{\epsilon}$. This set consists of $2^{\ell}$ generators. 
The complex $\operatorname{CF}(\Sigma, \boldsymbol{\alpha}, \boldsymbol{\beta}, \mathbf{z} ; \mathfrak{s})$ is thus trivial for $\mathfrak{s} \neq \mathfrak{s}_{0}$ and is equal to

$$
\mathrm{CF}\left(\Sigma, \boldsymbol{\alpha}, \boldsymbol{\beta}, \mathbf{w} ; \mathfrak{s}_{0}\right) \otimes_{\mathbb{A}_{\mu}} \mathbb{A}_{\tau}
$$

for $\mathfrak{s}=\mathfrak{s}_{0}$ where $\mathbf{w}=\left\{z_{1}^{1}, z_{1}^{2}, \ldots, z_{1}^{m}\right\}$. For the rest of the computation, we may thus assume that $\mathbf{w}=\mathbf{z}$, i.e. that there is a single marked point in each connected component of $\Sigma-\boldsymbol{\alpha}$. Each $\mu_{i}$ (or under the assumption $\mathbf{w}=\mathbf{z}$, each $\lambda_{i}$ ) corresponds to some component $A_{i}$. Thus $\mu_{i}=0$ if the genus of $A_{i}$ is positive. With our previous notation, this means that $\mu_{k+1}=\ldots=\mu_{m}=0$. We set the degree associated with $\mu_{i}, i=1, \ldots, k$, equal to $-2=\left\langle c_{1}\left(\mathfrak{s}_{0}\right), H\left(A_{i}\right)\right\rangle$. This gives a grading on the complex $\mathrm{CF}\left(\Sigma, \boldsymbol{\alpha}, \boldsymbol{\beta}, \mathbf{w} ; \mathfrak{s}_{0}\right)$.

Let us assume that $\epsilon, \delta:\{1, \ldots, \ell\} \rightarrow\{-,+\}$ are a pair of indices. After re-naming the elements of $\{1, \ldots, \ell\}$ we may assume that

$$
\begin{cases}\epsilon(i)=+\& \delta(i)=- & \text { if } 1 \leq i \leq \ell_{1} \\ \epsilon(i)=-\& \delta(i)=+ & \text { if } \ell_{1}<i \leq \ell_{2} \\ \epsilon(i)=\delta(i) & \text { if } \ell_{2}<i \leq \ell,\end{cases}
$$

for some $1 \leq \ell_{1} \leq \ell_{2} \leq \ell$. Then $\mathcal{D}=D_{1}^{+}+\ldots+D_{\ell_{1}}^{+}-D_{\ell_{1}+1}^{+}-\ldots-D_{\ell_{2}}^{+}$is the domain of a disk connecting $\mathbf{x}^{\epsilon}$ to $\mathbf{x}^{\delta}$. If $\mathcal{P}$ is a periodic domain and $\mathcal{D}+\mathcal{P}$ is the domain of a positive disk $\phi$ with $\lambda(\phi) \neq 0$, the same argument as before implies that

$$
\mathcal{P}=a_{1} A_{1}+\ldots+a_{k} A_{k}+q_{1} \mathcal{P}_{1}+\ldots+q_{\ell} \mathcal{P}_{\ell} .
$$

Furthermore, the assumption $\mathcal{D}(\phi) \geq 0$ implies that all $a_{i}$ are non-negative. In order to prove that the above grading assignment is well-defined, one only needs to check the following easy equality

$$
\mu(\phi)=2\left(\sum_{i=1}^{k} a_{i}\right)+2 \ell_{1}-\ell_{2} .
$$

If the coefficient ring $\widehat{\mathbb{A}}_{\tau}$ is used instead of $\mathbb{A}_{\tau}$, the corresponding quotient $\widehat{\mathbb{A}}_{\mu}$ of $\mathbb{A}_{\mu}$ will be equal to $\mathbb{Z}$. One would then quickly conclude from the above presentation of the domain $\mathcal{P}$ that if $\lambda(\phi) \neq 0$ (as an element in the quotient $\widehat{\mathbb{A}}_{\tau}$ ), then $a_{1}=\ldots=a_{k}=0$ and $\ell_{2}=\ell_{1}$. If $\mu(\phi)=1$ then $\ell_{1}=\ell_{2}=1$. We can then carry out the rest of the argument for any choice of indices $\epsilon$ and $\delta$, if the coefficient ring is replaced with $\widehat{\mathbb{A}}_{\tau}$.

With coefficients in $\mathbb{A}$, however, in order to complete our investigation we need to assume $\epsilon(i)=\{+\}$ for $i=1, \ldots, \ell$. The corresponding generator is often called the top generator. In this case the equality $\ell_{1}=\ell_{2}$ is automatic. Replacing $\mu(\phi)=1$ in equation 13 we obtain $a_{1}=\ldots=a_{k}=0$ and $\ell_{1}=1$. From here we will have (from positivity of the domain) that $q_{2}=\ldots=q_{\ell}=0$. This means that if the top intersection point $\mathbf{x}^{\epsilon}$ is connected to an intersection point $\mathbf{x}^{\delta}$ by a positive domain $\phi$ of index 1 , such that $\lambda(\phi) \neq 0, \delta$ differs from $\epsilon$ only over one element of $\{1, \ldots, \ell\}$, where $\epsilon$ gives + and $\delta$ gives - . Let us assume that this element is $i \in\{1, \ldots, \ell\}$.

If $i \neq 1$, the possible domains one may obtain as $\mathcal{D}+\mathcal{P}$ are $D_{i}^{+}$and $D_{i}^{-}$, and the total contribution of $\mathbf{x}^{\delta}$ to $\partial\left(\mathbf{x}^{\epsilon}\right)$ is zero. However, if $i=1$, the possible domains are $D_{1}^{+}, D_{1}^{-}+D_{2}^{-}$and $D_{1}^{-}+D_{2}^{+}$. Again, the total contribution of these three domains 
is zero by the argument of OS5 (lemma 9.4). The above discussion implies that the top generator $\mathbf{x}^{\epsilon}$ is closed and represents a non-trivial element of the homology groups corresponding to either of chain complexes

$$
\mathrm{CF}\left(\Sigma, \boldsymbol{\alpha}, \boldsymbol{\beta}, \mathbf{w} ; \mathfrak{s}_{0}\right) \quad \& \quad \mathrm{CF}\left(\Sigma, \boldsymbol{\alpha}, \boldsymbol{\beta}, \mathbf{z} ; \mathfrak{s}_{0}\right)=\mathrm{CF}\left(\Sigma, \boldsymbol{\alpha}, \boldsymbol{\beta}, \mathbf{z} ; \mathfrak{s}_{0}\right) \otimes_{\mathbb{A}_{\mu}} \mathbb{A}_{\tau} .
$$

Moreover, the same argument implies that all the generators of the form $\mathbf{x}^{\delta}$ are closed, when the coefficient ring $\widehat{\mathbb{A}}_{\tau}$ is used instead of $\mathbb{A}_{\tau}$, giving rise to an identification of the chain complexes:

$$
\mathrm{CF}\left(\Sigma, \boldsymbol{\alpha}, \boldsymbol{\beta}, \mathbf{z} ; \mathfrak{s}_{0} ; \widehat{\mathbb{A}}\right)=\widehat{\mathrm{HF}}\left(\#^{\ell} S^{1} \times S^{2}, \mathfrak{t}_{0}\right) \otimes_{\mathbb{Z}} \widehat{\mathbb{A}}_{\tau} .
$$

The above equality means that the differential on the right hand side of the above equality is trivial. Any module isomorphism of the right hand side which respects the filtration by relative $\operatorname{Spin}^{c}$ structures is thus a filtered chain homotopy equivalence. The top generator $\mathbf{x}^{\epsilon}$ of $\operatorname{CF}\left(\Sigma, \boldsymbol{\alpha}, \boldsymbol{\beta}, \mathbf{z} ; \mathfrak{s}_{0}\right)$ is usually denoted by $\Theta$, or $\Theta_{\alpha \beta}$.

The above example illustrates how the arguments of Ozsváth and Szabó for the study of the special Heegaard diagrams, i.e. Heegaard diagrams where most of $\beta_{i}$ are Hamiltonian isotopes of curves in $\boldsymbol{\alpha}$, may be generalized to the present situation. The above type of Heegaard diagrams appear in the arguments for the invariance under handle-slide. We will face similar Heegaard diagrams again. In particular this happens when we study the exact triangles. Each time, a separate argument should be presented for computing the contribution of holomorphic disks and polygons. However the argument is always a straight forward modification of the corresponding argument for Heegaard diagrams arising from closed threemanifolds.

\subsection{The triangle map and the invariance. Fix a Heegaard triple}

$$
H=(\Sigma, \boldsymbol{\alpha}, \boldsymbol{\beta}, \boldsymbol{\gamma}, \mathbf{z})
$$

and assume that $\mathbf{z}=\left\{z_{1}, \ldots, z_{\kappa}\right\}$. We will denote the balanced sutured manifold associated with $(\Sigma, \boldsymbol{\alpha}, \boldsymbol{\beta}, \mathbf{z})$ by $(X, \tau)=\left(X_{\alpha \beta}, \tau_{\alpha \beta}\right)$, and the corresponding coefficient ring by $\mathbb{A}=\mathbb{A}_{\tau}$. Similarly, let $\left(X_{\alpha \gamma}, \tau_{\alpha \gamma}\right)$ and $\left(X_{\beta \gamma}, \tau_{\beta \gamma}\right)$ be the balanced sutured manifolds associated with the Heegaard diagrams $(\Sigma, \boldsymbol{\alpha}, \boldsymbol{\gamma}, \mathbf{z})$, and $(\Sigma, \boldsymbol{\beta}, \boldsymbol{\gamma}, \mathbf{z})$ respectively. Suppose that

$$
\Sigma-\boldsymbol{\alpha}=\coprod_{i=1}^{k} A_{i}, \quad \Sigma-\boldsymbol{\beta}=\coprod_{i=1}^{l} B_{i}, \quad \& \quad \Sigma-\gamma=\coprod_{i=1}^{m} C_{i},
$$

where $A_{i}, B_{i}$ and $C_{i}$ are the connected components of the curve complements. We will furthermore assume that $m=l$, and that these components are labeled so that for each $i=1, \ldots, l$ we have $C_{i} \cap \mathbf{z}=B_{i} \cap \mathbf{z}$, and $g\left(C_{i}\right)=g\left(B_{i}\right)$. This implies that $\lambda(\boldsymbol{\beta})=\lambda(\boldsymbol{\gamma})$ in $\left\langle\lambda_{1}, \ldots, \lambda_{\kappa}\right\rangle_{\mathbb{Z}}$, and more importantly, $\lambda\left(C_{i}\right)=\lambda\left(B_{i}\right)$ for $i=1, \ldots, l$.

Assume that the coefficient rings $\mathbb{A}_{\beta \gamma}$ and $\mathbb{A}_{\alpha \gamma}$ are associated with the Heegaard diagrams $(\Sigma, \boldsymbol{\beta}, \boldsymbol{\gamma}, \mathbf{z})$ and $(\Sigma, \boldsymbol{\alpha}, \boldsymbol{\gamma}, \mathbf{z})$ respectively. Then the above observation implies that

$$
\mathbb{A}_{\beta \gamma}=\frac{\left\langle\lambda_{1}, \ldots, \lambda_{\kappa}\right\rangle_{\mathbb{Z}}}{\left\langle\lambda\left(B_{i}\right) \mid g\left(B_{i}\right)=g\left(C_{i}\right)>0\right\rangle}
$$


is naturally mapped by a quotient homomorphism

$$
\rho_{\beta \gamma}: \mathbb{A}_{\beta \gamma} \longrightarrow \mathbb{A}_{\alpha \beta}=\mathbb{A}_{\tau}
$$

to $\mathbb{A}$. We may thus consider the $\mathbb{A}$-module

$$
\mathrm{CF}(\Sigma, \boldsymbol{\beta}, \boldsymbol{\gamma}, \mathbf{z}) \otimes_{\mathbb{A}_{\beta \gamma}} \mathbb{A},
$$

which will have the structure of a filtered $(\mathbb{A}, \mathbb{H})$ chain complex, with $\mathbb{H}=\mathrm{H}^{2}(X, \partial X ; \mathbb{Z})$.

Any triangle class $\psi_{H} \in \operatorname{Spin}^{c}(H,\{\boldsymbol{\alpha}, \boldsymbol{\beta}, \gamma\})$ determines a set of three $\operatorname{Spin}^{c}$ structures

$$
\mathfrak{s}_{\alpha \beta} \in \operatorname{Spin}^{c}\left(\overline{X_{\alpha \beta}}\left(\tau_{\alpha \beta}\right)\right), \quad \mathfrak{s}_{\alpha \gamma} \in \operatorname{Spin}^{c}\left(\overline{X_{\alpha \gamma}}\left(\tau_{\alpha \gamma}\right)\right), \quad \& \mathfrak{s}_{\beta \gamma} \in \operatorname{Spin}^{c}\left(\overline{X_{\beta \gamma}}\left(\tau_{\beta \gamma}\right)\right) .
$$

These three $\operatorname{Spin}^{c}$ classes, together with the triangle class of $\psi_{H}$ give a coherent system of $\operatorname{Spin}^{c}$ structures for $H$ which will be denoted by $\mathfrak{T}$. We will assume that $\mathfrak{T}$, or equivalently the triangle class $\psi_{H}$, is fixed, and will drop them from the notation when there is no confusion. In particular, by the admissibility of a Heegaard diagram we would mean $\mathfrak{T}$-admissibility.

Any choice of coherent systems of orientations $\mathfrak{o}_{\alpha \beta}$ and $\mathfrak{o}_{\alpha \gamma}$ associated with the $\operatorname{Spin}^{c}$ classes $\mathfrak{s}_{\alpha \beta}$ and $\mathfrak{s}_{\alpha \gamma}$ may be completed to a coherent system of orientations for $\mathfrak{T}$ by lemma 6.6. Furthermore, we are free to choose the orientation associated with a fixed representative of $\psi_{H}$. Let us fix such a coherent system $\mathfrak{o}$ of orientations. Once again, we will drop this choice of orientation from the notation.

Assuming that the Heegaard triple $H$ is admissible, the triangle map corresponding to $H$ (and the triangle class $\psi_{H}$ ) is defined via the construction of subsection 6.1.

$$
\begin{aligned}
& f_{\alpha \beta \gamma}: \mathrm{CF}\left(\Sigma, \boldsymbol{\alpha}, \boldsymbol{\beta}, \mathbf{z} ; \mathfrak{s}_{\alpha \beta}\right) \otimes\left(\mathrm{CF}\left(\Sigma, \boldsymbol{\beta}, \boldsymbol{\gamma}, \mathbf{z} ; \mathfrak{s}_{\beta \gamma}\right) \otimes_{\mathbb{A}_{\beta \gamma}} \mathbb{A}_{\tau}\right) \rightarrow \mathrm{CF}\left(\Sigma, \boldsymbol{\alpha}, \boldsymbol{\gamma}, \mathbf{z} ; \mathfrak{s}_{\alpha \gamma}\right) \\
& f_{\alpha \beta \gamma}(\mathbf{x} \otimes \mathbf{q})=\sum_{\mathbf{y} \in \mathbb{T}_{\alpha} \cap \mathbb{T}_{\gamma}} \sum_{\substack{\psi \in \pi_{2}^{0}(\mathbf{x}, \mathbf{q}, \mathbf{y}) \\
(\psi)=\left(\psi_{H}\right)}}\left(\mathfrak{m}(\psi) \lambda_{\mathbf{z}}(\psi)\right) \cdot \mathbf{y}
\end{aligned}
$$

As usual, $\lambda_{\mathbf{z}}$ is the map

$$
\begin{aligned}
& \lambda_{\mathbf{z}}: \coprod_{\mathbf{x} \in \mathbb{T}_{\alpha} \cap \mathbb{T}_{\beta}} \coprod_{\mathbf{y} \in \mathbb{T}_{\beta} \cap \mathbb{T}_{\gamma}} \coprod_{\mathbf{w} \in \mathbb{T}_{\gamma} \cap \mathbb{T}_{\alpha}} \pi_{2}^{+}(\mathbf{x}, \mathbf{y}, \mathbf{w}) \longrightarrow G(\mathbb{A}) \\
& \lambda_{\mathbf{z}}(\psi):=\prod_{i=1}^{\kappa} \lambda_{i}^{n_{z_{i}}(\psi)} \in G(\mathbb{A}) .
\end{aligned}
$$

The admissibility of the Heegaard diagram implies that $f_{\alpha \beta \gamma}$ is well-defined.

Lemma 6.11. The map $f_{\alpha \beta \gamma}$ is an $\mathbb{A}$ chain map. More precisely

$$
f_{\alpha \beta \gamma}(\partial(\mathbf{x}) \otimes \mathbf{q})+f_{\alpha \beta \gamma}(\mathbf{x} \otimes \partial(\mathbf{q}))=\partial\left(f_{\alpha \beta \gamma}(\mathbf{x} \otimes \mathbf{q})\right)
$$

for all $\mathbf{x} \in \mathbb{T}_{\alpha} \cap \mathbb{T}_{\beta}$ and $\mathbf{q} \in \mathbb{T}_{\beta} \cap \mathbb{T}_{\gamma}$ corresponding to the $\operatorname{Spin}^{c}$ classes $\mathfrak{s}_{\alpha \beta}$ and $\mathfrak{s}_{\beta \gamma}$ respectively. 
Proof. The equality

$$
f_{\alpha \beta \gamma}(\partial(\mathbf{x}) \otimes \mathbf{q})+f_{\alpha \beta \gamma}(\mathbf{x} \otimes \partial(\mathbf{q}))=\partial\left(f_{\alpha \beta \gamma}(\mathbf{x} \otimes \mathbf{q})\right)
$$

in the above lemma is nothing but the following special case (i.e. the case $m=3$ ) of theorem 6.8.

$$
f_{\alpha \beta \gamma}\left(f_{\alpha \beta}(\mathbf{x}) \otimes \mathbf{q}\right)+f_{\alpha \beta \gamma}\left(\mathbf{x} \otimes f_{\beta \gamma}(\mathbf{q})\right)-f_{\alpha \gamma}\left(f_{\alpha \beta \gamma}(\mathbf{x} \otimes \mathbf{q})\right)=0 .
$$

As in OS5, holomorphic triangle maps satisfy an associativity law, which comes from considering Heegaard quadruples. Let $K=(\Sigma, \boldsymbol{\alpha}, \boldsymbol{\beta}, \boldsymbol{\gamma}, \boldsymbol{\delta}, \mathbf{z})$ be an admissible Heegaard quadruple. This means that we have a coherent system $\mathfrak{T}$ of $\operatorname{Spin}^{c}$ classes of polygons, which consists of a square class

$$
\psi_{K} \in \operatorname{Spin}^{c}(K,\{\boldsymbol{\alpha}, \boldsymbol{\beta}, \boldsymbol{\gamma}, \boldsymbol{\delta}\})
$$

and triangle classes

$$
\begin{aligned}
& \psi_{\alpha} \in \operatorname{Spin}^{c}(K,\{\boldsymbol{\beta}, \boldsymbol{\gamma}, \boldsymbol{\delta}\}), \quad \psi_{\beta} \in \operatorname{Spin}^{c}(K,\{\boldsymbol{\alpha}, \boldsymbol{\gamma}, \boldsymbol{\delta}\}) \\
& \psi_{\gamma} \in \operatorname{Spin}^{c}(K,\{\boldsymbol{\alpha}, \boldsymbol{\beta}, \boldsymbol{\delta}\}), \quad \& \quad \psi_{\delta} \in \operatorname{Spin}^{c}(K,\{\boldsymbol{\alpha}, \boldsymbol{\beta}, \boldsymbol{\gamma}\}) .
\end{aligned}
$$

We implicitly assume that the set of corners of these representatives of the triangle classes is a fixed set of 6 intersection points between the pairs of tori from $\left\{\mathbb{T}_{\alpha}, \mathbb{T}_{\beta}, \mathbb{T}_{\gamma}, \mathbb{T}_{\delta}\right\}$. These classes have to satisfy the following compatibility criteria

$$
\psi_{K}=\psi_{\alpha} \star \psi_{\gamma}=\psi_{\beta} \star \psi_{\delta} .
$$

The triangle classes also determine $\operatorname{Spin}^{c}$ structures on the Heegaard diagrams determined by any pair of curve collections. These $\operatorname{Spin}^{c}$ structures will be denoted by $\mathfrak{s}_{\alpha \beta} \in \operatorname{Spin}^{c}\left(\overline{X_{\alpha \beta}}\left(\tau_{\alpha \beta}\right)\right)$, etc.. Moreover, we will assume that

$$
\Sigma-\boldsymbol{\alpha}=\coprod_{i=1}^{k} A_{i}, \quad \Sigma-\boldsymbol{\beta}=\coprod_{i=1}^{l} B_{i}, \quad \Sigma-\boldsymbol{\gamma}=\coprod_{i=1}^{l} C_{i}, \quad \& \quad \Sigma-\boldsymbol{\delta}=\coprod_{i=1}^{l} D_{i},
$$

are labeled so that $B_{i} \cap \mathbf{z}=C_{i} \cap \mathbf{z}=D_{i} \cap \mathbf{z}$ for $i=1, \ldots, l$. Furthermore, we will assume that $g\left(B_{i}\right)=g\left(C_{i}\right)=g\left(D_{i}\right)$ for $i=1, \ldots, l$. Then we will have $\lambda(\boldsymbol{\beta})=\lambda(\boldsymbol{\gamma})=\lambda(\boldsymbol{\delta})$ in $\left\langle\lambda_{1}, \ldots, \lambda_{\kappa}\right\rangle$, and $\lambda\left(B_{i}\right)=\lambda\left(C_{i}\right)=\lambda\left(D_{i}\right)$ for $i=1, \ldots, l$.

One may also choose a coherent system of orientations associated with $\mathfrak{T}$. In fact, we are free to choose $\mathfrak{o}_{\alpha \beta}, \mathfrak{o}_{\alpha \gamma}, \mathfrak{o}_{\alpha \delta}$, and the orientation of the triangle classes $\psi_{\beta}, \psi_{\gamma}$ and $\psi_{\delta}$. Once again, we keep such a coherent system of orientations implicit in our notation.

We may thus consider the following filtered $(\mathbb{A}, \mathbb{H})$ chain complexes, which are relevant for the associativity:

$$
\begin{aligned}
C_{\alpha \beta}=\mathrm{CF}\left(\Sigma, \boldsymbol{\alpha}, \boldsymbol{\beta}, \mathbf{z} ; \mathfrak{s}_{\alpha \beta}\right), & & C_{\beta \gamma}=\mathrm{CF}\left(\Sigma, \boldsymbol{\beta}, \boldsymbol{\gamma}, \mathbf{z} ; \mathfrak{s}_{\beta \gamma}\right) \otimes \mathbb{A}_{\tau} \\
C_{\alpha \gamma}=\mathrm{CF}\left(\Sigma, \boldsymbol{\alpha}, \boldsymbol{\gamma}, \mathbf{z} ; \mathfrak{s}_{\alpha \gamma}\right), & & C_{\beta \delta}=\mathrm{CF}\left(\Sigma, \boldsymbol{\beta}, \boldsymbol{\delta}, \mathbf{z} ; \mathfrak{s}_{\beta \delta}\right) \otimes \mathbb{A}_{\tau} \\
C_{\alpha \delta}=\mathrm{CF}\left(\Sigma, \boldsymbol{\alpha}, \boldsymbol{\delta}, \mathbf{z} ; \mathfrak{s}_{\alpha \delta}\right), & \& & C_{\gamma \delta}=\mathrm{CF}\left(\Sigma, \boldsymbol{\gamma}, \boldsymbol{\delta}, \mathbf{z} ; \mathfrak{s}_{\gamma \delta}\right) \otimes \mathbb{A}_{\tau} .
\end{aligned}
$$


Following the construction of subsection 6.1 we define a rectangle map as in OS5:

$$
\begin{aligned}
& h_{\alpha \beta \gamma \delta}: C_{\alpha \beta} \otimes C_{\beta \gamma} \otimes C_{\gamma \delta} \longrightarrow C_{\alpha \delta} \\
& h_{\alpha \beta \gamma \delta}(\mathbf{x} \otimes \mathbf{p} \otimes \mathbf{q})=\sum_{\mathbf{y} \in \mathbb{T}_{\alpha} \cap \mathbb{T}_{\delta}} \sum_{\substack{\psi \in \pi_{2}(\mathbf{x}, \mathbf{p}, \mathbf{q}, \mathbf{y}) \\
\mu(\psi)=-1}}(\mathfrak{m}(\psi) \lambda(\psi)) \mathbf{y} .
\end{aligned}
$$

Lemma 6.12. The rectangle map $h_{\alpha \beta \gamma \delta}$ gives a chain homotopy between the chain maps $f_{\alpha \gamma \delta}\left(f_{\alpha \beta \gamma}(. \otimes.) \otimes.\right)$ and $f_{\alpha \beta \delta}\left(. \otimes f_{\beta \gamma \delta}(. \otimes).\right)$ in the sense that

$$
\begin{aligned}
f_{\alpha \gamma \delta}( & \left(f_{\alpha \beta \gamma}(\mathbf{x} \otimes \mathbf{p}) \otimes \mathbf{q}\right)-f_{\alpha \beta \delta}\left(\mathbf{x} \otimes f_{\beta \gamma \delta}(\mathbf{p} \otimes \mathbf{q})\right) \\
\quad & =\partial\left(h_{\alpha \beta \gamma \delta}(\mathbf{x} \otimes \mathbf{p} \otimes \mathbf{q})\right)+h_{\alpha \beta \gamma \delta}(\partial(\mathbf{x} \otimes \mathbf{p} \otimes \mathbf{q}))
\end{aligned}
$$

for any $\mathbf{x} \in \mathbb{T}_{\alpha} \cap \mathbb{T}_{\beta}, \mathbf{p} \in \mathbb{T}_{\beta} \cap \mathbb{T}_{\gamma}$, and $\mathbf{q} \in \mathbb{T}_{\gamma} \cap \mathbb{T}_{\delta}$.

Proof. Once again, this is a special case of theorem 6.8, where we put $m=4$ and use the above data.

Proof. Theorem 5.9 The proof of the independence from the choice of the path of almost complex structures, as well as the proof of the isotopy invariance of the filtered $(\mathbb{A}, \mathbb{H})$ chain homotopy type of $\operatorname{CF}(\Sigma, \boldsymbol{\alpha}, \boldsymbol{\beta}, \mathbf{z} ; \mathfrak{s})$ is the same as the proof of the special case discussed in OS5]. We only need to keep track of the marked points, and that the constructed chain homotopy equivalence respects the decomposition into relative $\operatorname{Spin}^{c}$ classes in $\operatorname{Spin}^{c}(X, \tau)$. The same is almost true for the handle slides supported away from the marked points. We will present the proof in this case, to give an illustration of the procedure, which involves the use of holomorphic triangles and squares introduced above.

Fix a $\operatorname{Spin}^{c}$ class $\mathfrak{s} \in \operatorname{Spin}^{c}(\bar{X})$ and let $\mathfrak{s} \in \mathfrak{s} \subset \operatorname{Spin}^{c}(X, \tau)$ be a fixed relative $\operatorname{Spin}^{c}$ class in $\mathfrak{s}$. To prove the handle slide invariance consider the Heegaard quadruple $(\Sigma, \boldsymbol{\alpha}, \boldsymbol{\beta}, \boldsymbol{\gamma}, \boldsymbol{\delta}, \mathbf{z})$ where $\boldsymbol{\gamma}$ and $\boldsymbol{\delta}$ are obtained from $\boldsymbol{\beta}$ as follows. Let $\boldsymbol{\beta}=\left\{\beta_{1}, \ldots, \beta_{\ell}\right\}$. Then we let $\delta_{i}$ to be a small Hamiltonian isotope of $\beta_{i}$ for $i=1, \ldots, \ell$ which cuts it in a pair of transverse canceling intersection points. Similarly, for $i=2, \ldots, \ell$, we let $\gamma_{i}$ be a small Hamiltonian isotope of $\beta_{i}$ which cuts either of the curves $\beta_{i}$ and $\delta_{i}$ in a pair of transverse canceling intersection points. Finally, we let $\gamma_{1}$ be the simple closed curve obtained by first moving $\beta_{1}$ by a small Hamiltonian isotopy, and then taking its handle slide over $\beta_{2}$. We may assume that $\gamma_{1}$ cuts either of $\beta_{1}$ and $\delta_{1}$ in a pair of canceling intersection points, while it is disjoint from the rest of the curves $\beta_{i}, \gamma_{i}$ and $\delta_{i}$. We let

$$
\gamma=\left\{\gamma_{1}, \ldots, \gamma_{\ell}\right\}, \quad \& \quad \delta=\left\{\delta_{1}, \ldots, \delta_{\ell}\right\} .
$$

Consider the (admissible) Heegaard diagram $(\Sigma, \boldsymbol{\beta}, \boldsymbol{\gamma}, \mathbf{z})$, which is a standard Heegaard diagram of the type studied in subsection 6.2 Note that all marked points which are in the same connected component of $\Sigma-\boldsymbol{\beta}$ or $\Sigma-\boldsymbol{\gamma}$ are in the same connected component of $\Sigma-\boldsymbol{\beta}-\boldsymbol{\gamma}$. Let $\Theta_{\beta \gamma}$ be the top generator of the complex

$$
\mathrm{CF}\left(\Sigma, \boldsymbol{\beta}, \boldsymbol{\gamma}, \mathbf{z} ; \mathfrak{s}_{0}\right)
$$

corresponding to its canonical $\mathrm{Spin}^{c}$ structure. This generator is represented by the intersection point in $\mathbb{T}_{\beta} \cap \mathbb{T}_{\gamma}$ which contains positive intersection points between the 
corresponding curves $\beta_{i}$ and $\gamma_{i}$. Similarly, associated with the Heegaard diagram $(\Sigma, \boldsymbol{\beta}, \boldsymbol{\delta}, \mathbf{z})$ the top generator of the homology is denoted by a $\Theta_{\beta \delta}$, and is represented by the positive intersection points between the corresponding curves $\beta_{i}$ and $\delta_{i}$. Finally, $\Theta_{\gamma \delta}$ is defined in a similar way. We may consider $\Theta_{\beta \gamma}, \Theta_{\gamma \delta}$ and $\Theta_{\beta \delta}$ as generators of the complexes $C_{\beta \gamma}, C_{\gamma \delta}$ and $C_{\beta \delta}$ respectively. Here, we assume $\mathfrak{s}_{\alpha} \bullet \mathfrak{s}$ for $\bullet \in\{\beta, \gamma, \delta\}$, and that

$$
\mathfrak{s}_{\beta \gamma}=\mathfrak{s}_{\beta \delta}=\mathfrak{s}_{\gamma \delta}=\mathfrak{s}_{0}
$$

is the canonical $\operatorname{Spin}^{c}$ structure on $\overline{X_{\beta \gamma}}=\overline{X_{\gamma \delta}}=\overline{X_{\beta \delta}}$. Note that $\Theta_{\beta \gamma}, \Theta_{\gamma \delta}$ and $\Theta_{\beta \delta}$ are connected to each other by a natural triangle class $\Delta_{\alpha}$ of small area. Moreover, for any fixed $\mathbf{x} \in \mathbb{T}_{\alpha} \cap \mathbb{T}_{\beta}$ with $\mathfrak{s}(\mathbf{x}) \in \mathfrak{s}$, we have a generator $I(\mathbf{x}) \in \mathbb{T}_{\alpha} \cap \mathbb{T}_{\delta}$, determined by the closest intersection points in $\mathbb{T}_{\alpha} \cap \mathbb{T}_{\delta}$ to $\mathbf{x}$. Similarly, there is a generator $J(\mathbf{x})$ in $\mathbb{T}_{\alpha} \cap \mathbb{T}_{\gamma}$ determined as the closest intersection points between $\boldsymbol{\alpha}$ and $\boldsymbol{\gamma}$ to $\mathbf{x}$. There is a triangle class $\Delta_{\gamma}$ connecting $\Theta_{\beta \delta}, \mathbf{x}$ and $I(\mathbf{x})$ with very small area. Similarly, there is a triangle class $\Delta_{\delta}$ connecting $\Theta_{\beta \gamma}, \mathbf{x}$ and $J(\mathbf{x})$ with very small area. Finally, there is a triangle class $\Delta_{\beta}$ which connects $I(\mathbf{x}), J(\mathbf{x})$ and $\Theta_{\gamma \delta}$. Let $\square$ be the square class $\Delta_{\gamma} \star \Delta_{\alpha}$. Then $\square$ may also be degenerated as $\square=\Delta_{\delta} \star \Delta_{\beta}$. The data

$$
\mathfrak{P}=\left\{\square, \Delta_{\alpha}, \Delta_{\beta}, \Delta_{\gamma}, \Delta_{\delta}\right\}
$$

thus gives a coherent system $\mathfrak{T}$ of $\operatorname{Spin}^{c}$ classes of polygons for the Heegaard quadruple, which will be implicit for the rest of the construction.

Lemma 6.13. The Heegaard quadruple $H=(\Sigma, \boldsymbol{\alpha}, \boldsymbol{\beta}, \boldsymbol{\gamma}, \boldsymbol{\delta}, \mathbf{z})$ is $\mathfrak{T}$-admissible, provided that $H=(\Sigma, \boldsymbol{\alpha}, \boldsymbol{\beta}, \mathbf{z})$ is $\mathfrak{s}$-admissible.

Proof. We will prove the lemma for the class of $\square$. The rest of the admissibility claims are similar, and in fact simpler. Let us denote the small periodic domains constructed as the domain bounded between $\beta_{i}$ and $\delta_{i}$ by $\mathcal{Q}_{i}$ for $i=1, \ldots, \ell$. Thus $\mathcal{Q}_{i}$ is the difference of two bi-gons. Similarly, let $\mathcal{Q}_{i+\ell}$ be the domain bounded between $\beta_{i}$ and $\gamma_{i}$ for $i=2, \ldots, \ell$, and $\mathcal{Q}_{\ell+1}$ be the domain bounded between $\beta_{1}, \gamma_{1}$ and $\beta_{2}$, as in the previous subsection. We will thus have

$$
\begin{cases}\partial \mathcal{Q}_{i}=\beta_{i}-\delta_{i}, & \text { for } i=1, \ldots, \ell, \\ \partial \mathcal{Q}_{i+\ell}=\beta_{i}-\gamma_{i}, & \text { for } i=2, \ldots, \ell, \& \\ \partial \mathcal{Q}_{\ell+1}=\beta_{1}+\beta_{2}-\gamma_{1} . & \end{cases}
$$

Finally, let $A_{1}, \ldots, A_{k}, B_{1}, \ldots, B_{l}, \mathcal{P}_{1}, \ldots, \mathcal{P}_{m}$ be the periodic domains corresponding to $(\Sigma, \boldsymbol{\alpha}, \boldsymbol{\beta}, \mathbf{z})$. As before $\Sigma-\boldsymbol{\alpha}=\coprod A_{i}$ and $\Sigma-\boldsymbol{\beta}=\coprod B_{i}$. It may be checked then that the space of periodic domains for the Heegaard diagrams $(\Sigma, \boldsymbol{\beta}, \boldsymbol{\gamma}, \mathbf{z})$, $(\Sigma, \boldsymbol{\beta}, \boldsymbol{\delta}, \mathbf{z})$ and $(\Sigma, \boldsymbol{\gamma}, \boldsymbol{\delta}, \mathbf{z})$ is generated by the following periodic domains respectively

$$
\begin{aligned}
& \left\langle B_{1}, \ldots, B_{l}, \mathcal{Q}_{\ell+1}, \ldots, \mathcal{Q}_{2 \ell}\right\rangle, \quad\left\langle B_{1}, \ldots, B_{l}, \mathcal{Q}_{1}, \ldots, \mathcal{Q}_{\ell}\right\rangle, \& \\
& \left\langle\widehat{B}_{1}, \ldots, \widehat{B}_{l}, \mathcal{Q}_{1}+\mathcal{Q}_{2}-\mathcal{Q}_{\ell+1}, \mathcal{Q}_{2}-\mathcal{Q}_{2+\ell}, \ldots, \mathcal{Q}_{\ell}-\mathcal{Q}_{2 \ell}\right\rangle .
\end{aligned}
$$

Here, $\widehat{B}_{i}$ is the domain obtained from $B_{i}$ by adding an appropriated combination of $\mathcal{Q}_{j}, j=1, \ldots, \ell$, so that its boundary is supported on the curves in $\boldsymbol{\delta}$. 
Let us now assume that we have a periodic domain $\mathcal{P}$ with

$$
\begin{aligned}
& \mathcal{P}=\mathcal{P}_{\alpha \beta}+\mathcal{P}_{\beta \gamma}+\mathcal{P}_{\gamma \delta}+\mathcal{P}_{\alpha \delta} \geq 0, \quad \lambda(\mathcal{P}) \neq 0, \quad \& \\
& \left\langle c_{1}(\mathfrak{s}), H\left(\mathcal{P}_{\alpha \beta}\right)\right\rangle+\left\langle c_{1}\left(\mathfrak{t}_{0}\right), H\left(\mathcal{P}_{\beta \gamma}\right)\right\rangle+\left\langle c_{1}\left(\mathfrak{t}_{0}\right), H\left(\mathcal{P}_{\gamma \delta}\right)\right\rangle+\left\langle c_{1}(\mathfrak{s}), H\left(\mathcal{P}_{\alpha \delta}\right)\right\rangle=0
\end{aligned}
$$

$\mathcal{P}$ may then be written as

$$
\mathcal{P}=\sum_{i=1}^{k} a_{i} A_{i}+\sum_{i=1}^{l} b_{i} B_{i}+\sum_{i=1}^{m} p_{i} \mathcal{P}_{i}+\sum_{i=1}^{2 \ell} q_{i} \mathcal{Q}_{i} .
$$

With the above notation fixed, computing the evaluation of $\operatorname{Spin}^{c}$ classes over the periodic domains (i.e. re-writing the last equation above) we obtain

$$
0=\sum_{i=1}^{k} a_{i}\left(2-2 g\left(A_{i}\right)\right)+\sum_{i=1}^{l} b_{i}\left(2-2 g\left(B_{i}\right)\right)+\sum_{i=1}^{m} p_{i}\left\langle c_{1}(\mathfrak{s}), H\left(\mathcal{P}_{i}\right)\right\rangle,
$$

since the Maslov index of all $\mathcal{Q}_{i}$ are zero for all $\operatorname{Spin}^{c}$ structures, according to Lipshitz' index formula Lip. Let us set

$$
\mathcal{Q}=\sum_{i=1}^{k} a_{i} A_{i}+\sum_{i=1}^{l} b_{i} B_{i}+\sum_{i=1}^{m} p_{i} \mathcal{P}_{i}
$$

Then $\mathcal{Q}$ is a periodic domain for the Heegaard diagram $(\Sigma, \boldsymbol{\alpha}, \boldsymbol{\beta}, \mathbf{z})$, with $\mathcal{P}-\mathcal{Q}$ only consisting of the domains with very small area. The assumption $\mathcal{P} \geq 0$ thus implies that $\mathcal{Q} \geq 0$. Furthermore $\lambda(\mathcal{Q})=\lambda(\mathcal{P})$, since no marked point lives in the small domains. The equation 14 implies that $\left\langle c_{1}(\mathfrak{s}), H(\mathcal{Q})\right\rangle=0$. The $\mathfrak{s}$-admissibility of of the Heegaard diagram $(\Sigma, \boldsymbol{\alpha}, \boldsymbol{\beta}, \mathbf{z})$ thus implies that $\mathcal{Q}=0$. As a result,

$$
\mathcal{P}=\sum_{i=1}^{2 \ell} q_{i} \mathcal{Q}_{i} \geq 0
$$

It is then an easy combinatorial exercise to check from this last equality that all $q_{i}$ need to vanish. We have thus shown that $\mathcal{P}=0$. This completes the proof of the admissibility claim.

Finally, the last step towards defining the holomorphic triangle map and the holomorphic square map using the Heegaard diagram $H$ is choosing the orientation. Note that the choice of orientation over the Heegaard diagrams $(\Sigma, \boldsymbol{\alpha}, \boldsymbol{\beta}, \mathbf{z})$, $(\Sigma, \boldsymbol{\alpha}, \boldsymbol{\gamma}, \mathbf{z})$, and $(\Sigma, \boldsymbol{\alpha}, \boldsymbol{\delta}, \mathbf{z})$ may be done without any restriction, and we may thus choose the orientation on $(\Sigma, \boldsymbol{\alpha}, \boldsymbol{\beta}, \mathbf{z})$, and the induced orientations on the other Heegaard diagrams as our preferred choice of orientation. Orienting the triangles and the square in $\mathfrak{T}$ will then provide us with a coherent system $\mathfrak{o}$ of orientations for the Heegaard diagram $H$.

We may thus define the triangle and the square maps associated with this Heegaard diagram and T. The argument of Ozsváth and Szabó from OS5] (lemma 9.7) applies here to give

$$
f_{\beta \gamma \delta}\left(\Theta_{\beta \gamma} \otimes \Theta_{\gamma \delta}\right)=\Theta_{\beta \delta} .
$$

We may thus define a map

$$
F=F_{\alpha \beta \gamma}: \mathrm{CF}(\Sigma, \boldsymbol{\alpha}, \boldsymbol{\beta}, \mathbf{z} ; \mathfrak{s}) \longrightarrow \mathrm{CF}(\Sigma, \boldsymbol{\alpha}, \boldsymbol{\gamma}, \mathbf{z} ; \mathfrak{s})
$$


by setting $F(\mathbf{x}):=f_{\alpha \beta \gamma}\left(\mathbf{x} \otimes \Theta_{\beta \gamma}\right)$. Since $\Theta_{\beta \gamma}$ is closed, $F$ is a chain map. More importantly, $F$ respects the decomposition into relative $\operatorname{Spin}^{c}$ structures, and the image of $\operatorname{CF}(\Sigma, \boldsymbol{\alpha}, \boldsymbol{\beta}, \mathbf{z} ; \mathfrak{s})$, for the fixed relative $\operatorname{Spin}^{c}$ structure

$$
\underline{\mathfrak{s}} \in \mathfrak{s} \subset \operatorname{Spin}^{c}\left(X_{\alpha \beta}, \tau_{\alpha \beta}\right)=\operatorname{Spin}^{c}(X, \tau)
$$

is in $\operatorname{CF}(\Sigma, \boldsymbol{\alpha}, \boldsymbol{\gamma}, \mathbf{z} ; \underline{\mathfrak{s}})$. Let us denote by $G$ the similar filtered $(\mathbb{A}, \mathbb{H})$ chain map

$$
G=F_{\alpha \gamma \delta}: \mathrm{CF}(\Sigma, \boldsymbol{\alpha}, \boldsymbol{\gamma}, \mathbf{z} ; \mathfrak{s}) \longrightarrow \mathrm{CF}(\Sigma, \boldsymbol{\alpha}, \boldsymbol{\delta}, \mathbf{z} ; \mathfrak{s})
$$

defined by $G(\mathbf{y}):=f_{\alpha \gamma \delta}\left(\mathbf{y} \otimes \Theta_{\gamma \delta}\right)$. Also, define the map

$$
H=H_{\alpha \beta \gamma \delta}: \mathrm{CF}(\Sigma, \boldsymbol{\alpha}, \boldsymbol{\beta}, \mathbf{z} ; \mathfrak{s}) \longrightarrow \mathrm{CF}(\Sigma, \boldsymbol{\alpha}, \boldsymbol{\delta}, \mathbf{z} ; \mathfrak{s})
$$

by $H(\mathbf{x}):=h_{\alpha \beta \gamma \delta}\left(\mathbf{x} \otimes \Theta_{\beta \gamma} \otimes \Theta_{\gamma \delta}\right)$. Checking that all the above maps respect the relative $\operatorname{Spin}^{c}$ structures is straight forward. Using lemma 6.12, and the fact that $f_{\beta \gamma \delta}\left(\Theta_{\beta \gamma} \otimes \Theta_{\gamma \delta}\right)=\Theta_{\beta \delta}$ we have

$$
G(F(\mathbf{x})))-f_{\alpha \beta \delta}\left(\mathbf{x} \otimes \Theta_{\beta \delta}\right)=\partial(H(\mathbf{x}))+H(\partial(\mathbf{x})) .
$$

Small triangles which contribute to $f_{\alpha \beta \delta}\left(\mathbf{x} \otimes \Theta_{\beta \delta}\right)$ may be used to show that in terms of an appropriate energy filtration we have

$$
f_{\alpha \beta \delta}\left(\mathbf{x} \otimes \Theta_{\beta \delta}\right)=I(x)+\epsilon(\mathbf{x})
$$

where $\epsilon(\mathbf{x})$ consists of a combination of generators with smaller energy than $\mathbf{x}$. This implies that there is a filtered $(\mathbb{A}, \mathbb{H})$ chain equivalence

$$
K: \mathrm{CF}(\Sigma, \boldsymbol{\alpha}, \boldsymbol{\delta}, \mathbf{z} ; \mathfrak{s}) \longrightarrow \mathrm{CF}(\Sigma, \boldsymbol{\alpha}, \boldsymbol{\beta}, \mathbf{z} ; \mathfrak{s}),
$$

respecting the decomposition according to relative $\operatorname{Spin}^{c}$ structures, such that $K\left(f_{\alpha \beta \delta}\left(\mathbf{x} \otimes \Theta_{\beta \delta}\right)\right)=\mathbf{x}$. Thus setting $G^{\prime}=K \circ G$ and $H^{\prime}=K \circ H$ we have

$$
G^{\prime} \circ F-I d=H^{\prime} \circ \partial+\partial \circ H^{\prime},
$$

and $G^{\prime} \circ F$ is chain homotopic to the identity. The other composition is similarly chain homotopic to the identity. This completes the proof of the handle slide invariance.

The invariance under isotopy and stabilization-destabilization is completely similar to the proofs presented in sections 7 and 10 of OS5. Thus the filtered $(\mathbb{A}, \mathbb{H})$ chain homotopy type of $\mathrm{CF}(\Sigma, \boldsymbol{\alpha}, \boldsymbol{\beta}, \mathbf{z} ; \mathfrak{s})$ is an invariant of $(X, \tau, \mathfrak{s})$, and will be denoted by

$$
\mathrm{CF}(X, \tau ; \mathfrak{s})=\bigoplus_{\underline{\mathfrak{s}} \in \mathfrak{s}} \mathrm{CF}(X, \tau ; \mathfrak{\mathfrak { s }})
$$

Theorem 5.9, together with remark 4.3 imply that for computing $\mathrm{CF}(X, \tau ; \mathfrak{s} ; \mathbb{B})$, given a test ring $\mathbb{B}$ for $\mathbb{A}_{\tau}$, one may use any Heegaard diagram which is weakly $\mathfrak{s - a d m i s s i b l e ~ i n ~ t h e ~ s e n s e ~ o f ~ r e m a r k ~ 4 . 3 . ~ I n ~ p a r t i c u l a r , ~ w e ~ c a n ~ e a s i l y ~ p r o v e ~ t h e ~}$ following proposition.

Proposition 6.14. The irreducible balanced sutured manifold $(X, \tau)$ is taut if and only if the filtered $\left(\mathbb{B}_{\tau}, \mathbb{H}_{\tau}\right)$ chain homotopy type of the complex $\mathrm{CF}\left(X, \tau ; \mathfrak{s} ; \mathbb{B}_{\tau}\right)$ is not trivial for some $\operatorname{Spin}^{c}$ structure $\mathfrak{s} \in \operatorname{Spin}^{c}(\bar{X})$. 
Proof. Suppose that $(X, \tau)$ is an irreducible balanced sutured manifold which is not taut. As in the proof of proposition 9.18 from [Ju1, there is a weakly admissible Heegaard diagram $(\Sigma, \boldsymbol{\alpha}, \boldsymbol{\beta}, \mathbf{z})$ for $(X, \tau)$ such that $\mathbb{T}_{\alpha} \cap \mathbb{T}_{\beta}$ is empty. This Heegaard diagram is $\mathfrak{s}$-admissible for the test ring $\rho_{\tau}: \mathbb{A}_{\tau} \rightarrow \mathbb{B}_{\tau}$ and for any $\mathfrak{s} \in \operatorname{Spin}^{c}(\bar{X})$ by remark 4.3 and may thus be used to compute

$$
\mathrm{CF}\left(X, \tau ; \mathfrak{s} ; \mathbb{B}_{\tau}\right) \cong 0, \quad \forall \mathfrak{s} \in \operatorname{Spin}^{c}(\bar{X}),
$$

where $\cong$ denotes the equivalence of filtered chain homotopy types.

Conversely, if $(X, \tau)$ is taut, theorem 1.4 from $\mathrm{Ju} 2$ implies that $\operatorname{HF}(X, \tau ; \mathbb{Z}) \neq 0$. Since $\mathbb{Z}$ is a test ring for $\mathbb{B}_{\tau}$ this implies, in particular, that the filtered chain homotopy type of $\mathrm{CF}\left(X, \tau ; \mathbb{B}_{\tau}\right)$ is non-trivial.

In fact, the proof of proposition 6.14 implies the following corollary.

Corollary 6.15. For an irreducible balanced sutured manifold $(X, \tau)$, the filtered $\left(\mathbb{B}_{\tau}, \mathbb{H}_{\tau}\right)$ chain homotopy type of $\mathrm{CF}\left(X, \tau ; \mathbb{B}_{\tau}\right)$ is trivial if and only if $\operatorname{SFH}(X, \tau)=$ 0 . 


\section{Stabilization of SUtured MANifolds}

7.1. Simple stabilization of a balanced sutured manifold. Let us fix a balanced sutured manifold $\left(X, \tau=\left\{\gamma_{1}, \ldots, \gamma_{\kappa}\right\}\right)$ and let

$$
\mathfrak{R}^{-}(\tau)=\bigcup_{i=1}^{k} R_{i}^{-}, \& \mathfrak{R}^{+}(\tau)=\bigcup_{j=1}^{l} R_{j}^{+},
$$

as before.

Definition 7.1. We say that a sutured manifold $(X, \widehat{\tau})$ is obtained by a simple stabilization of $(X, \tau)$ if $\widehat{\tau}=\tau \cup\left\{\gamma_{\kappa+1}, \gamma_{\kappa+2}\right\}$ and $\gamma_{\kappa+1}$ and $\gamma_{\kappa+2}$ are oriented simple closed curves so that $-\gamma_{\kappa+1}$ and $\gamma_{\kappa+2}$ are both parallel to an oriented suture $\gamma_{i} \in \tau$, where $\gamma_{i}$ is in the common boundary of two genus zero components of $\mathfrak{R}(\tau)$. Moreover, $\gamma_{i}$ and $\gamma_{\kappa+1}$ bound an annulus in $\partial X-\widehat{\tau}$.

Without loss of generality we assume that $i=\kappa$, and that $\gamma_{i}=\gamma_{\kappa} \in \partial R_{k}^{-} \cap \partial R_{l}^{+}$ and $\gamma_{\kappa+1}, \gamma_{\kappa+2} \subset R_{l}^{+}$. So the genera of both $R_{k}^{-}$and $R_{l}^{+}$are zero. Note that

$$
\begin{array}{ll} 
& \mathfrak{R}^{-}(\widehat{\tau})=\mathfrak{R}^{-}(\tau) \coprod A_{\kappa+1, \kappa+2}, \quad \& \\
& \mathfrak{R}^{+}(\widehat{\tau})=\left(\mathfrak{R}^{+}(\tau)-R_{l}^{+}\right) \coprod A_{\kappa, \kappa+1} \coprod R_{l+1}^{+} \\
\text {where } & R_{l}^{+}-\left(\gamma_{\kappa+1} \cup \gamma_{\kappa+2}\right)=A_{\kappa+1, \kappa+2} \coprod A_{\kappa, \kappa+1} \coprod R_{l+1}^{+}
\end{array}
$$

and $A_{\kappa+1, \kappa+2}$ and $A_{\kappa, \kappa+1}$ are the annulus components with the boundary sets $\left\{\gamma_{\kappa+1}, \gamma_{\kappa+2}\right\}$ and $\left\{\gamma_{\kappa}, \gamma_{\kappa+1}\right\}$ respectively. Thus we have

$$
\begin{aligned}
& \lambda^{+}(\widehat{\tau})=\lambda^{+}(\tau)+\lambda_{\kappa} \lambda_{\kappa+1}+\lambda_{l+1}^{+}-\lambda_{l}^{+}, \& \\
& \lambda^{-}(\widehat{\tau})=\lambda^{-}(\tau)+\lambda_{\kappa+1} \lambda_{\kappa+2}
\end{aligned}
$$

where $\lambda_{l+1}^{+}=\lambda\left(R_{l+1}^{+}\right)$. The algebra associated to the boundary of $(X, \widehat{\tau})$ is defined by

$$
\mathbb{A}_{\widehat{\tau}}=\frac{\left\langle\lambda_{1}, \ldots, \lambda_{\kappa}, \lambda_{\kappa+1}, \lambda_{\kappa+2}\right\rangle_{\mathbb{Z}}}{\left\langle\lambda^{+}(\widehat{\tau})-\lambda^{-}(\widehat{\tau})\right\rangle_{\mathbb{Z}[\kappa+2]}+\left\langle\lambda_{i}^{+} \mid g_{i}^{+}>0\right\rangle_{\mathbb{Z}[\kappa+2]}+\left\langle\lambda_{j}^{-} \mid g_{j}^{-}>0\right\rangle_{\mathbb{Z}[\kappa+2]}}
$$

Note that the homomorphism

$$
\begin{aligned}
& f: \mathbb{Z}\left[\lambda_{1}, \ldots, \lambda_{\kappa+2}\right] \longrightarrow \mathbb{Z}\left[\lambda_{1}, \ldots, \lambda_{\kappa+1}\right] \\
& f\left(\lambda_{i}\right)= \begin{cases}\lambda_{i} & \text { if } i \neq \kappa+2 \\
\lambda_{\kappa} & \text { if } i=\kappa+2\end{cases}
\end{aligned}
$$

induces a homomorphism $\widehat{f}: \mathbb{A}_{\widehat{\tau}} \longrightarrow \mathbb{A}_{\tau}\left[\lambda_{\kappa+1}\right]$, where

$$
\mathbb{A}_{\tau}\left[\lambda_{\kappa+1}\right]=\mathbb{A}_{\tau} \otimes_{\mathbb{Z}\left[\lambda_{1}, \ldots, \lambda_{\kappa}\right]} \mathbb{Z}\left[\lambda_{1}, \ldots, \lambda_{\kappa+1}\right] .
$$

Consequently, $\left(\mathbb{A}_{\tau}\left[\lambda_{\kappa+1}\right], \widehat{f}\right)$ is a test ring for $\mathbb{A}_{\widehat{\tau}}$.

Let $(\Sigma, \boldsymbol{\alpha}, \boldsymbol{\beta}, \mathbf{z})$ be a Heegaard diagram for $(X, \tau)$. A Heegaard diagram $(\Sigma, \widehat{\boldsymbol{\alpha}}, \widehat{\boldsymbol{\beta}}, \widehat{\mathbf{z}})$ for $(X, \widehat{\tau})$ may then be constructed from $(\Sigma, \boldsymbol{\alpha}, \boldsymbol{\beta}, \mathbf{z})$ as follows. We set

$$
\widehat{\boldsymbol{\alpha}}=\boldsymbol{\alpha} \cup\left\{\alpha_{\ell+1}\right\}, \& \widehat{\boldsymbol{\beta}}=\boldsymbol{\beta} \cup\left\{\beta_{\ell+1}\right\}, \& \widehat{\mathbf{z}}=\mathbf{z} \cup\left\{z_{\kappa+1}, z_{\kappa+2}\right\},
$$

where the additional curves $\alpha_{\ell+1}$ and $\beta_{\ell+1}$ are isotopic simple closed curves on $\Sigma$ in the the domain of $\Sigma-\boldsymbol{\alpha} \cup \boldsymbol{\beta}$ containing the marked point $z_{\kappa}$ with the following 
properties. We assume that $\# \alpha_{\ell+1} \cap \beta_{\ell+1}=2$ and that $\alpha_{\ell+1}$ and $\beta_{\ell+1}$ bound the disks $A_{k+1}$ and $B_{l+1}$ respectively. Furthermore, we assume that

$$
z_{\kappa} \in B_{l+1}-A_{k+1}, \quad z_{\kappa+1} \in A_{k+1} \cap B_{l+1}, \quad \& \quad z_{\kappa+2} \in A_{k+1}-B_{l+1} .
$$

The picture around the marked point $z_{\kappa}$ is illustrated in figure 2

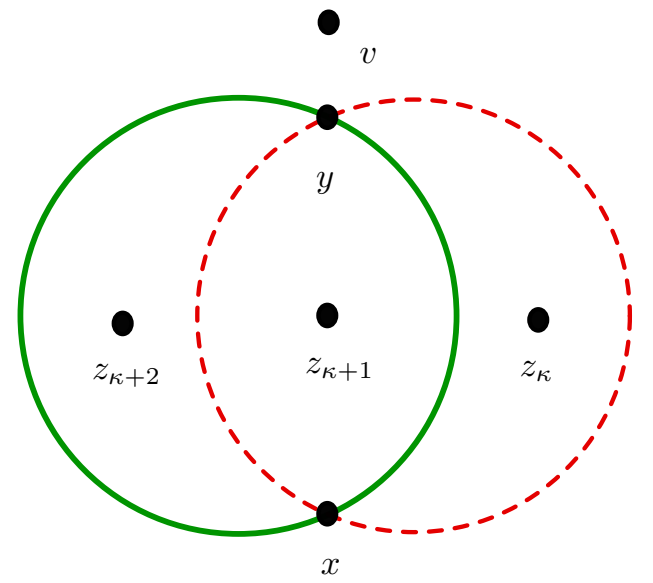

Figure 2. For simple stabilization, a pair of intersecting nullhomotopic simple closed curves $\alpha_{\ell+1}$ and $\beta_{\ell+1}$ are added to the Heegaard diagram close to the marked point $z_{\kappa}$. The locations of the new marked points $z_{\kappa}, z_{\kappa+1}$ and $z_{\kappa+2}$ are illustrated in the figure. The marked point $v$ is used as the connected sum point of the current diagram (on a Riemann sphere) with the old Heegaard diagram.

Note that $H\left(A_{k+1}\right)=\left[S_{\kappa+1, \kappa+2}\right]$ and $H\left(B_{l+1}\right)=\left[S_{\kappa, \kappa+1}\right]$ in $H_{2}\left(\bar{X}_{\widehat{\tau}}=\bar{X}^{\widehat{\tau}}\right)$ where $S_{\kappa, \kappa+1}$ and $S_{\kappa+1, \kappa+2}$ are sphere boundary components of $\bar{X}_{\widehat{\tau}}$ corresponding to $A_{\kappa, \kappa+1}$ and $A_{\kappa+1, \kappa+2}$ in $\Re(\widehat{\tau})$ respectively. In the above situation,we say the Heegaard diagram $(\Sigma, \widehat{\boldsymbol{\alpha}}, \widehat{\boldsymbol{\beta}}, \widehat{\mathbf{z}})$ is obtained from $(\Sigma, \boldsymbol{\alpha}, \boldsymbol{\beta}, \mathbf{z})$ by a simple stabilization.

We may define a natural map

$$
\widehat{\imath}: \operatorname{Spin}^{c}(X, \widehat{\tau}) \longrightarrow \operatorname{Spin}^{c}(X, \tau)
$$

as follows. Fix $\widehat{\underline{\mathfrak{s}}} \in \operatorname{Spin}^{c}(X, \widehat{\tau})$ and let $\widehat{v}$ be a nowhere vanishing vector field on $X$ representing $\underline{\underline{\mathfrak{s}}}$ such that $\left.\widehat{v}\right|_{\partial X}=v_{\widehat{\tau}}$. Consider a neighborhood $N$ of

$$
A=\bar{A}_{\kappa, \kappa+1} \cup \bar{A}_{\kappa+1, \kappa+2} \subset \partial X
$$

together with a diffeomorphism

$$
\begin{aligned}
& \psi: N \longrightarrow S^{1} \times I \times I, \quad \text { s.t. } \\
& \psi(A)=S^{1} \times\{0\} \times I,\left.\quad \& \quad \psi_{*}\left(\left.\widehat{v}\right|_{N}\right)\right|_{S^{1} \times I \times\{0,1\}}=\frac{\partial}{\partial s},
\end{aligned}
$$

where $I=[0,1]$ is the unit interval and $s$ denotes the standard parameter on the third component of the product $S^{1} \times I \times I$. The vector field $\psi_{*}(\widehat{v})$ may be changed 
through an isotopy to a new vector field $\psi_{*}(v)$ on $S^{1} \times I \times I$ with the property $\left.\psi_{*}(v)\right|_{S^{1} \times\{0\} \times I}=\frac{\partial}{\partial s}$, where the vector field remains fixed through the isotopy on

$$
\left(S^{1} \times\{1\} \times I\right) \bigcup\left(S^{1} \times I \times\{0\}\right) \bigcup\left(S^{1} \times I \times\{1\}\right) .
$$

The vector field $v$ on $N$ may be glued to $\left.\widehat{v}\right|_{X-N}$ to give a vector field on $X$, still denote by $v$, which represents an element $\underline{\mathfrak{s}}$ in $\operatorname{Spin}^{c}(X, \tau)$. Define $\widehat{\imath}(\underline{\mathfrak{s}}):=\underline{\mathfrak{s}}$. It is easy to see that $\widehat{\imath}$ is well-defined and bijective, and that the following diagram is commutative

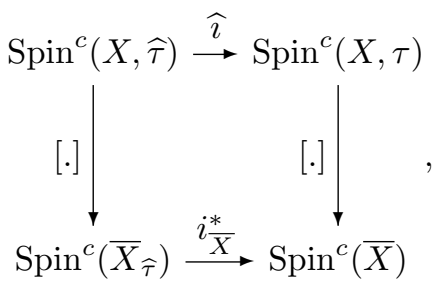

where $i_{\bar{X}}: \bar{X} \rightarrow \bar{X}_{\widehat{\tau}}$ denotes the inclusion map.

Proposition 7.2. Let $(X, \widehat{\tau})$ be a sutured manifold obtained by a simple stabilization on $(X, \tau)$ and $(\Sigma, \widehat{\boldsymbol{\alpha}}, \widehat{\boldsymbol{\beta}}, \widehat{\mathbf{z}})$ be the Heegaard diagram for $(X, \widehat{\tau})$ obtained by the simple stabilization of the Heegaard diagram $(\Sigma, \boldsymbol{\alpha}, \boldsymbol{\beta}, \mathbf{z})$ for $(X, \tau)$ as above. Then for any $\operatorname{Spin}^{c}$ class $\mathfrak{s} \in \operatorname{Spin}^{c}\left(\bar{X}_{\widehat{\tau}}\right)$ the filtered chain homotopy type of the complex

$$
\mathrm{CF}\left(\Sigma, \widehat{\boldsymbol{\alpha}}, \widehat{\boldsymbol{\beta}}, \widehat{\mathbf{z}}, \mathfrak{s} ; \mathbb{A}_{\tau}\left[\lambda_{\kappa+1}\right]\right)=\mathrm{CF}(\Sigma, \widehat{\boldsymbol{\alpha}}, \widehat{\boldsymbol{\beta}}, \widehat{\mathbf{z}}, \mathfrak{s}) \otimes_{\mathbb{A}_{\widehat{\tau}}} \mathbb{A}_{\tau}\left[\lambda_{\kappa+1}\right]
$$

is the same as the filtered chain homotopy type of the mapping cone of

$$
\mathrm{CF}\left(\Sigma, \boldsymbol{\alpha}, \boldsymbol{\beta}, \mathbf{z}, i_{\bar{X}}^{*}(\mathfrak{s}) ; \mathbb{A}_{\tau}\left[\lambda_{\kappa+1}\right]\right) \stackrel{\lambda_{\kappa+1}-\lambda}{\longrightarrow} \mathrm{CF}\left(\Sigma, \boldsymbol{\alpha}, \boldsymbol{\beta}, \mathbf{z}, i_{\bar{X}}^{*}(\mathfrak{s}) ; \mathbb{A}_{\tau}\left[\lambda_{\kappa+1}\right]\right) .
$$

where we define

$$
\begin{aligned}
\mathrm{CF}\left(\Sigma, \boldsymbol{\alpha}, \boldsymbol{\beta}, \mathbf{z}, i_{\bar{X}}^{*}(\mathfrak{s}) ; \mathbb{A}_{\tau}\left[\lambda_{\kappa+1}\right]\right) & :=\mathrm{CF}\left(\Sigma, \boldsymbol{\alpha}, \boldsymbol{\beta}, \mathbf{z}, i_{\bar{X}}^{*}(\mathfrak{s})\right) \otimes_{\mathbb{A}_{\tau}} \mathbb{A}_{\tau}\left[\lambda_{\kappa+1}\right], \\
\& \quad \lambda & :=\prod_{\gamma_{\kappa} \neq \gamma_{i} \in \partial R_{l}^{+}} \lambda_{i} \in \mathbb{A}_{\tau}\left[\lambda_{\kappa+1}\right] .
\end{aligned}
$$

7.2. The analytic input. Before we start the proving proposition 7.2 , we need to rephrase the statements of theorem 5.1, lemma 6.3 and lemma 6.4 of OS8 for balanced sutured manifolds and the corresponding Heegaard diagrams.

Let $(X, \tau)$ be a sutured manifold with the Heegaard diagram $(\Sigma, \boldsymbol{\alpha}, \boldsymbol{\beta}, \mathbf{z})$ and consider a point $v$ on $\Sigma$. Let $\phi \in \pi_{2}(\mathbf{x}, \mathbf{y})$ be the homotopy class of a Whitney disk connecting intersection points $\mathbf{x}$ and $\mathbf{y}$, and assume that $n_{v}(\phi)=k \in \mathbb{Z}^{\geq 0}$. We may define a map

$$
\begin{gathered}
\rho^{v}: \mathcal{M}(\phi) \longrightarrow \operatorname{Sym}^{k}(\mathbb{D}) \\
\rho^{v}(u)=u^{-1}\left(v \times \operatorname{Sym}^{\ell-1}(\Sigma)\right)
\end{gathered}
$$

Correspondingly, we may define the moduli spaces $\mathcal{M}(\phi, t)$ and $\mathcal{M}(\phi, \Delta)$ by

$$
\begin{aligned}
\mathcal{M}(\phi, t) & =\left\{u \in \mathcal{M}(\phi) \mid(t, 0) \in \rho^{v}(u)\right\}, \& \\
\mathcal{M}(\phi, \Delta) & =\left\{u \in \mathcal{M}(\phi) \mid \rho^{v}(u)=\Delta\right\}
\end{aligned}
$$


where $t \in[0,1]$ and $\Delta \in \operatorname{Sym}^{k}(\mathbb{D})$.

Let $\left(X_{1}, \tau_{1}\right)$ and $\left(X_{2}, \tau_{2}\right)$ be sutured manifolds with corresponding Heegaard diagrams $\left(\Sigma_{1}, \boldsymbol{\alpha}_{1}, \boldsymbol{\beta}_{1}, \mathbf{z}_{1}\right)$ and $\left(\Sigma_{2}, \boldsymbol{\alpha}_{2}, \boldsymbol{\beta}_{2}, \mathbf{z}_{2}\right)$. We can form their connected sum along the points $w$ and $v$ on $\Sigma_{1}$ and $\Sigma_{2}$ to obtain a new sutured manifold $(X, \tau)$ with the corresponding Heegaard diagram $(\Sigma, \boldsymbol{\alpha}, \boldsymbol{\beta}, \mathbf{z})$ where

$$
\Sigma=\Sigma_{1} \# \Sigma_{2}, \quad \boldsymbol{\alpha}=\boldsymbol{\alpha}_{1} \cup \boldsymbol{\alpha}_{2}, \quad \boldsymbol{\beta}=\boldsymbol{\beta}_{1} \cup \boldsymbol{\beta}_{2} \quad \& \quad \mathbf{z}=\mathbf{z}_{1} \cup \mathbf{z}_{2} .
$$

Note that $\mathbb{T}_{\alpha} \cap \mathbb{T}_{\beta}=\left(\mathbb{T}_{\alpha_{1}} \cap \mathbb{T}_{\beta_{1}}\right) \times\left(\mathbb{T}_{\alpha_{2}} \cap \mathbb{T}_{\beta_{2}}\right)$. Consider intersection points $\mathbf{x}_{1}, \mathbf{y}_{1} \in \mathbb{T}_{\alpha_{1}} \cap \mathbb{T}_{\beta_{1}}$ and $\mathbf{x}_{2}, \mathbf{y}_{2} \in \mathbb{T}_{\alpha_{2}} \cap \mathbb{T}_{\beta_{2}}$. Any homology class

$$
\phi \in \pi_{2}\left(\mathbf{x}_{1} \times \mathbf{x}_{2}, \mathbf{y}_{1} \times \mathbf{y}_{2}\right)
$$

can be uniquely decomposed as $\phi=\phi_{1} \# \phi_{2}$ where

$$
\phi_{1} \in \pi_{2}\left(\mathbf{x}_{1}, \mathbf{y}_{1}\right), \quad \phi_{2} \in \pi_{2}\left(\mathbf{x}_{2}, \mathbf{y}_{2}\right), \quad \& n_{w}\left(\phi_{1}\right)=n_{v}\left(\phi_{2}\right) .
$$

Conversely, any pair of homology classes $\phi_{1} \in \pi_{2}\left(\mathbf{x}_{1}, \mathbf{y}_{1}\right)$ and $\phi_{2} \in \pi_{2}\left(\mathbf{x}_{2}, \mathbf{y}_{2}\right)$ such that $n_{w}\left(\phi_{1}\right)=n_{v}\left(\phi_{2}\right)$ can be combined to a give homology class

$$
\phi=\phi_{1} \# \phi_{2} \in \pi_{2}\left(\mathbf{x}_{1} \times \mathbf{x}_{2}, \mathbf{y}_{1} \times \mathbf{y}_{2}\right) \text {. }
$$

Theorem 7.3. Let $\left(X_{1}, \tau_{1}\right)$ and $\left(X_{2}, \tau_{2}\right)$ be balanced sutured manifolds with the corresponding Heegaard diagrams $\left(\Sigma_{1}, \boldsymbol{\alpha}_{1}, \boldsymbol{\beta}_{1}, \mathbf{z}_{1}\right)$ and $\left(\Sigma_{2}, \boldsymbol{\alpha}_{2}, \boldsymbol{\beta}_{2}, \mathbf{z}_{2}\right)$ respectively. Consider the balanced sutured manifold $(X, \tau)$ obtained by taking the connected sum of the two Heegaard diagrams along $w$ and $v$ as described above. Furthermore, assume that $w$ (respectively, $v$ ) is in a genus zero connected component of either of $\Sigma_{1}-\boldsymbol{\alpha}_{1}$, and $\Sigma_{1}-\boldsymbol{\beta}_{1}$ (respectively, $\Sigma_{2}-\boldsymbol{\alpha}_{2}, \Sigma_{2}-\boldsymbol{\beta}_{2}$ ). For any homotopy class

$$
\phi=\phi_{1} \# \phi_{2} \in \pi_{2}\left(\mathbf{x}_{1} \times \mathbf{x}_{2}, \mathbf{y}_{1} \times \mathbf{y}_{2}\right)
$$

we then have

$$
\mu(\phi)=\mu\left(\phi_{1}\right)+\mu\left(\phi_{2}\right)-2 k
$$

where $\mathbf{x}_{1}, \mathbf{y}_{1} \in \mathbb{T}_{\alpha_{1}} \cap \mathbb{T}_{\beta_{1}}, \mathbf{x}_{2}, \mathbf{y}_{2} \in \mathbb{T}_{\alpha_{2}} \cap \mathbb{T}_{\beta_{2}}$ and $k=n_{w}\left(\phi_{1}\right)=n_{v}\left(\phi_{2}\right)$.

Suppose furthermore that $\mu\left(\phi_{1}\right)=1, \mu\left(\phi_{2}\right)=2 k$ and one of the following is true:

- At least one component of $\mathfrak{R}\left(\tau_{2}\right)$ has nonzero genus and $\lambda(\phi) \neq 0$.

- All the components of $\mathfrak{R}\left(\tau_{2}\right)$ are genus zero, and

$$
\ell_{2}=\left|\boldsymbol{\alpha}_{2}\right|=\left|\boldsymbol{\beta}_{2}\right|>g\left(\Sigma_{2}\right) .
$$

If the fibered product

$$
\mathcal{M}\left(\phi_{1}\right) \times_{\operatorname{Sym}^{k}(\mathbb{D})} \mathcal{M}\left(\phi_{2}\right)=\left\{u_{1} \times u_{2} \in \mathcal{M}\left(\phi_{1}\right) \times \mathcal{M}\left(\phi_{2}\right) \mid \rho^{w}\left(u_{1}\right)=\rho^{v}\left(u_{2}\right)\right\}
$$

of $\mathcal{M}\left(\phi_{1}\right)$ and $\mathcal{M}\left(\phi_{2}\right)$ is a smooth manifold, then by taking the length of the connected sum tube sufficiently large there is an identification of this moduli space with $\mathcal{M}(\phi)$.

Proof. The proof is similar to the proof of theorem 5.1 in OS8. As in that proof we use Lipshitz cylindrical formulation. However, we keep the same notation for the moduli spaces and the corresponding maps for the sake of simplicity.

The formula for Maslov index follows from excision principle for the linearized $\bar{\partial}$ operator, using cylindrical formulation Lip. For the second part of the theorem, 
if all components of $\mathfrak{R}(\tau)$ be genus zero and $\ell_{2}>g\left(\Sigma_{2}\right)$, the proof of theorem 5.1 from OS8 applies word by word. In the other case, the proof requires some modification, as follows. We drop the details and only highlight the differences. For more details, we refer the reader to [OS8].

Suppose that $\mathfrak{R}(\tau)$ has a component with nonzero genus. Consider a sequence of almost complex structures $\{J(t)\}_{t \in[1, \infty)}$ on $\Sigma_{1} \# \Sigma_{2}$, where $J(t)$ denotes the complex structure determined by a pair of generic complex structures $j_{1}$ and $j_{2}$ on $\Sigma_{1}$ and $\Sigma_{2}$, and by setting the neck-length equal to $t$. Let us assume that $\widetilde{\mathcal{M}}_{J(t)}(\phi) \neq \emptyset$ as $t \longrightarrow$ $\infty$. Consider a sequence of pseudo-holomorphic curves $\left\{u_{t}\right\}_{t \in \mathbb{Z}^{+}}$such that $u_{t} \in$ $\mathcal{M}_{J(t)}(\phi)$. Under the assumptions $\mu\left(\phi_{1}\right)=1$ and $\mu\left(\phi_{2}\right)=2 k$, and using Gromov compactness theorem, a subsequence of this sequence is weakly convergent to a pseudo holomorphic representative $u_{1}$ of $\phi_{1}$ and a broken flow-line representative of $\phi_{2}$. This broken flow-line can not contain any sphere bubblings, since otherwise our assumption on $\mathfrak{R}(\tau)$ implies that $\lambda\left(\phi_{2}\right)=0$, and thus $\lambda(\phi)=0$. Hence we may continue the argument of Ozsváth and Szabó from here there, and conclude that there is a component $u_{2}$ of this broken flow line such that $\widetilde{u}_{1}$ and $\widetilde{u}_{2}$ represents a pre-glued flow line, i.e. that

$$
\rho^{w}\left(u_{1}\right)=\rho^{v}\left(u_{2}\right) .
$$

Let $\phi_{2}^{\prime}$ be the homotopy class represented by $u_{2}$. If $\phi_{2}^{\prime} \neq \phi_{2}$ then the above Gromov limit contains boundary degenerations or other flow lines. The assumption $\lambda\left(\phi_{2}\right) \neq 0$ then implies that $\mu\left(\phi_{2}^{\prime}\right)<\mu\left(\phi_{2}\right)=2 k$. Let us consider the map

$$
\rho^{v}: \mathcal{M}\left(\phi_{2}^{\prime}\right) \longrightarrow \operatorname{Sym}^{k}(\mathbb{D}) .
$$

For any point $\Delta \in \operatorname{Sym}^{k}(\mathbb{D})$ the moduli space $\left(\rho^{v}\right)^{-1}(\Delta)$ will have the expected dimension equal to $\mu\left(\phi^{\prime}\right)-2 k<0$. Thus for a generic choice of $\Delta \in \operatorname{Sym}^{k}(\mathbb{D})$, this moduli space is empty. This observation implies that $\phi_{2}^{\prime}=\phi_{2}$.

Thus the Gromov limit of a sequence of holomorphic representative of $\phi$, as we stretch the neck, is a pre-glued flow line representing $\phi_{1}$ and $\phi_{2}$. Conversely, given a pre-glued flow line, one can obtain a pseudo-holomorphic representative of $\phi$ in $\mathcal{M}(\phi)$ by the gluing theorem of Lipshitz [Lip], as in the proof of theorem 5.1 from OS8. This completes the proof of theorem 7.3 .

Lemma 7.4. Let $(X, \tau)$ be a balanced sutured manifold represented by the Heegaard diagram $(\Sigma, \boldsymbol{\alpha}, \boldsymbol{\beta}, \mathbf{z})$. Let $\phi \in \pi_{2}(\mathbf{x}, \mathbf{y})$ be the homotopy class of a Whitney disk connecting the intersection point $\mathbf{x}$ to $\mathbf{y}$. Assume furthermore that $\mathcal{D}(\phi) \geq 0$ and that $\lambda(\phi) \neq 0$. If $\mu(\phi)=2$ then $\mathcal{M}(\phi, t)$ is generically a zero dimensional moduli space. Furthermore, there is a number $\epsilon>0$ such that for all $t \leq \epsilon$ the only non-empty such moduli spaces $\mathcal{M}(\phi, t)$ are the moduli spaces corresponding to $\phi \in$ $\pi_{2}(\mathbf{x}, \mathbf{x})$ where $\phi$ is obtained by splicing a boundary degeneration with Maslov index 2 corresponding to one of the genus zero components of $\mathfrak{R}^{+}(\tau)$ and a constant flow line. For any such moduli space we have

$$
\# \mathcal{M}(\phi, t)=\left\{\begin{array}{ll}
0 & \text { if } l=1 \\
1 & \text { if } l>0
\end{array} .\right.
$$


Proof. Given lemma 5.8, the proof is exactly the same as the proof of lemma 6.3 in OS8].

Lemma 7.5. Consider the Heegaard diagram $(S, \alpha, \beta, \mathbf{z})$, where $S=S^{2}$ is the Riemann sphere, and $\alpha$ and $\beta$ are simple closed curves on $S$ intersecting transversely in two points $\{x, y\}$, and $\mathbf{z}=\left\{z_{1}, z_{2}, z_{3}, z_{4}\right\}$ where there is one marked point in each one of the four connected components of $S-\alpha-\beta$. Fix a generic point $\Delta \in \operatorname{Sym}^{k}(\mathbb{D})$ for some positive integer $k$. Then we have

$$
\sum_{\substack{\phi \in \pi_{2}^{2 k}(a, a) \\ n_{z_{4}}(\phi)=0}} \# \mathcal{M}(\phi, \Delta)=1,
$$

for $a \in\{x, y\}$.

Proof. Using lemma 5.8, the proof is exactly the same as the proof of lemma 6.4 in OS8.

7.3. Proof of the stabilization formula. In this subsection we prove proposition 7.2 .

Proof. (of proposition 7.2). Let $\mathfrak{s} \in \operatorname{Spin}^{c}\left(\bar{X}_{\hat{\tau}}\right)$ be a $\operatorname{Spin}^{c}$ structure on $\bar{X}_{\hat{\tau}}$. Consider a Heegaard diagram $(\Sigma, \boldsymbol{\alpha}, \boldsymbol{\beta}, \mathbf{z})$ for $(X, \tau)$, which is $i_{X}^{*}(\mathfrak{s})$-admissible. Furthermore, assume that for any positive periodic domain $\mathcal{P}$ we have the following implication:

$$
\left\langle c_{1}\left(i_{\bar{X}}^{*}(\mathfrak{s})\right), H(\mathcal{P})\right\rangle \leq 0 \Rightarrow \lambda(\mathcal{P})=0 .
$$

The existence of such a Heegaard diagram is guaranteed by remark 4.6 . Let $\widehat{H}=$ $(\Sigma, \widehat{\boldsymbol{\alpha}}, \widehat{\boldsymbol{\beta}}, \widehat{\mathbf{z}})$ be the Heegaard diagram for $(X, \widehat{\tau})$ obtained by a simple stabilization on $(\Sigma, \boldsymbol{\alpha}, \boldsymbol{\beta}, \mathbf{z})$. We claim that this Heegaard diagram is $\mathfrak{s}$-admissible. Suppose that $\mathcal{P}$ is a positive periodic domain corresponding to $\widehat{H}$ such that $\left\langle c_{1}(\mathfrak{s}), H(\mathcal{P})\right\rangle=0$. Then there are integers $a$ and $b$, and a positive periodic domain $\mathcal{P}_{0}$ for $H$ such that

$$
\mathcal{P}=\mathcal{P}_{0}+a A_{k+1}+b B_{l+1} \& n_{z_{\kappa}}\left(\mathcal{P}_{0}\right)=n_{z_{\kappa+1}}\left(\mathcal{P}_{0}\right)=n_{z_{\kappa+2}}\left(\mathcal{P}_{0}\right) .
$$

Thus $\mathcal{P}_{0}$ may be viewed as a periodic domain associated with the Heegaard diagram $(\Sigma, \boldsymbol{\alpha}, \boldsymbol{\beta}, \mathbf{z})$. If $n_{z_{\kappa}}\left(\mathcal{P}_{0}\right)=d$ then

$$
\left\langle c_{1}(\mathfrak{s}),\left(i_{\bar{X}}\right)_{*} H\left(\mathcal{P}_{0}\right)\right\rangle=\left\langle c_{1}\left(i_{\bar{X}}^{*}(\mathfrak{s})\right), H\left(\mathcal{P}_{0}\right)\right\rangle+2 d .
$$

From here we may conclude

$$
\begin{aligned}
& \left\langle c_{1}(\mathfrak{s}),\left(i_{\bar{X}}\right)_{*} H\left(\mathcal{P}_{0}\right)\right\rangle=-2(a+b) \\
\Rightarrow & \left\langle c_{1}\left(i_{\bar{X}}^{*}(\mathfrak{s})\right), H\left(\mathcal{P}_{0}\right)\right\rangle=-2(a+b+d)=-n_{z_{\kappa+1}}\left(\mathcal{P}_{0}\right) \leq 0 .
\end{aligned}
$$

Since the Heegaard diagram $(\Sigma, \boldsymbol{\alpha}, \boldsymbol{\beta}, \mathbf{z})$ is $i_{\bar{X}}^{*}(\mathfrak{s})$-admissible in the stronger sense of remark 4.6. we conclude that $\lambda\left(\mathcal{P}_{0}\right)=0$ in $\widetilde{\mathbb{A}}_{\tau}$. The condition that $z_{\kappa}$ is in the genus zero components of $\Sigma-\boldsymbol{\alpha}$ and $\Sigma-\boldsymbol{\beta}$ then implies that $\lambda(\mathcal{P})=0$ in $\widetilde{\mathbb{A}}_{\widehat{\tau}}$. This proves the $\mathfrak{s}$-admissibility of the Heegaard diagram $\widehat{H}$.

Let us consider the Heegaard diagram $(\Sigma, \widehat{\boldsymbol{\alpha}}, \widehat{\boldsymbol{\beta}}, \widehat{\mathbf{z}})$ as the connected sum

$$
\left(\Sigma, \boldsymbol{\alpha}, \boldsymbol{\beta}, \mathbf{z} \cup\{w\}-\left\{z_{\kappa}\right\}\right) \#\left(S, \alpha_{\ell+1}, \beta_{\ell+1},\left\{v, z_{\kappa}, z_{\kappa+1}, z_{\kappa+2}\right\}\right),
$$


where $S$ is a sphere, $w$ and $v$ are the corresponding connected sum points such that $w$ is in the same domain as $z_{\kappa}$ in $(\Sigma, \boldsymbol{\alpha}, \boldsymbol{\beta}, \mathbf{z})$. If $\alpha_{\ell+1} \cap \beta_{\ell+1}=\{x, y\}$ then $\mathbb{T}_{\widehat{\alpha}} \cap \mathbb{T}_{\widehat{\beta}}=\left(\mathbb{T}_{\alpha} \cap \mathbb{T}_{\beta}\right) \times\{x, y\}$, and for any $\mathbf{x} \in \mathbb{T}_{\alpha} \cap \mathbb{T}_{\beta}$ we have

$$
\underline{\mathfrak{s}}(\mathbf{x})=\widehat{\imath}(\underline{\mathfrak{g}}(\mathbf{x} \times\{x\}))=\widehat{\imath}(\underline{\mathfrak{s}}(\mathbf{x} \times\{y\}))+\operatorname{PD}\left[\gamma_{\kappa+1}\right] .
$$

Let $C_{x}$ and $C_{y}$ be the submodules of

$$
\mathrm{CF}\left(\Sigma, \widehat{\boldsymbol{\alpha}}, \widehat{\boldsymbol{\beta}}, \widehat{\mathbf{z}}, \mathfrak{s} ; \mathbb{A}_{\tau}\left[\lambda_{\kappa+1}\right]\right)=\mathrm{CF}(\Sigma, \widehat{\boldsymbol{\alpha}}, \widehat{\boldsymbol{\beta}}, \widehat{\mathbf{z}}, \mathfrak{s}) \otimes_{\mathbb{A}_{\widehat{\tau}}} \mathbb{A}_{\tau}\left[\lambda_{\kappa+1}\right]
$$

generated by the intersection points containing $x$ and $y$ respectively. Thus we have a module splitting

$$
\mathrm{CF}\left(\Sigma, \widehat{\boldsymbol{\alpha}}, \widehat{\boldsymbol{\beta}}, \widehat{\mathbf{z}}, \mathfrak{s} ; \mathbb{A}_{\tau}\left[\lambda_{\kappa+1}\right]\right)=C_{x} \oplus C_{y} .
$$

First we consider the $C_{x}$-components of the differential of the complex on the generators of $C_{x}$. Let $\mathbf{x} \times\{x\}$ be a generator of $C_{x}$ and $\phi \in \pi_{2}(\mathbf{x} \times\{x\}, \mathbf{y} \times\{x\})$ be the homology class of a Whitney disk with $\mu(\phi)=1$. We may thus decompose $\phi$ as $\phi=\phi_{1} \# \phi_{2}$ where $\phi_{1} \in \pi_{2}(\mathbf{x}, \mathbf{y})$ and $\phi_{2} \in \pi_{2}(x, x)$. Theorem 7.3 then implies that

$$
\mu(\phi)=\mu\left(\phi_{1}\right)+\mu\left(\phi_{2}\right)-2 k=\mu\left(\phi_{1}\right)+2 n_{z_{\kappa+1}}\left(\phi_{2}\right),
$$

where $k=n_{w}\left(\phi_{1}\right)=n_{v}\left(\phi_{2}\right)$. If $\mathcal{M}(\phi) \neq \emptyset$ for long enough neck-length, then $\phi_{2}$ admits holomorphic representatives and $\mathcal{D}\left(\phi_{2}\right) \geq 0$. This implies that

$$
\mu\left(\phi_{2}\right)-2 n_{v}\left(\phi_{2}\right)=2 n_{z_{\kappa+1}}(\phi) \geq 0,
$$

and that the equality happens if and only if $n_{z_{\kappa+1}}(\phi)=0$. If $\mu\left(\phi_{2}\right)-2 n_{v}\left(\phi_{2}\right)>0$ then $\mu\left(\phi_{1}\right) \leq-1$ and $\mathcal{M}\left(\phi_{1}\right)$ is generically empty. Thus $n_{z_{\kappa+1}}\left(\phi_{2}\right)$ should be zero and $\mu\left(\phi_{2}\right)=2 n_{v}\left(\phi_{2}\right)=2 k$. Theorem 7.3 then guarantees that for a sufficiently large connected sum length, we have an identification of $\mathcal{M}(\phi)$.

$$
\begin{aligned}
\mathcal{M}(\phi) & =\mathcal{M}\left(\phi_{1}\right) \times \times_{\operatorname{Sym}^{k}(\mathbb{D})} \mathcal{M}\left(\phi_{2}\right) \\
& =\left\{u_{1} \times u_{2} \in \mathcal{M}\left(\phi_{1}\right) \times \mathcal{M}\left(\phi_{2}\right) \mid \rho^{w}\left(u_{1}\right)=\rho^{v}\left(u_{2}\right)\right\} \\
\Rightarrow \# \widehat{\mathcal{M}}(\phi) & =\sum_{u_{1} \in \widehat{\mathcal{M}}\left(\phi_{1}\right)} \#\left\{u_{2} \in \mathcal{M}\left(\phi_{2}\right) \mid \rho^{w}\left(u_{1}\right)=\rho^{v}\left(u_{2}\right)\right\} .
\end{aligned}
$$

The coefficient of $\mathbf{y} \times\{x\}$ in the expression $\partial(\mathbf{x} \times\{x\})$ in $\mathrm{CF}\left(\Sigma, \widehat{\boldsymbol{\alpha}}, \widehat{\boldsymbol{\beta}}, \widehat{\mathbf{z}}, \mathfrak{s} ; \mathbb{A}_{\tau}\left[\lambda_{\kappa+1}\right]\right)$ is thus equal to

$$
\sum_{\substack{\phi_{1} \in \pi_{2}^{1}(\mathbf{x}, \mathbf{y}) \\ \phi_{2} \in \pi_{2}(x, x) \\ n_{z_{\kappa+1}}\left(\phi_{2}\right)=0}} \sum_{u_{1} \in \widehat{\mathcal{M}}\left(\phi_{1}\right)} \epsilon\left(u_{1}\right)\left(\prod_{i=1}^{\kappa} \lambda_{i}^{n_{z_{i}}\left(\phi_{1}\right)}\right) \#\left\{u_{2} \in \mathcal{M}\left(\phi_{2}\right) \mid \rho^{w}\left(u_{1}\right)=\rho^{v}\left(u_{2}\right)\right\},
$$

where $\epsilon\left(u_{1}\right)$ denotes the sign associated with $u_{1} \in \widehat{\mathcal{M}}\left(\phi_{1}\right)$ via a coherent system of orientations for the Heegaard diagram (which is suppressed from the notation). Lemma 7.5 may be used to compute the interior sum. The total value of the above sum is thus equal to

$$
\sum_{\phi_{1} \in \pi_{2}^{1}(\mathbf{x}, \mathbf{y})} \sum_{u_{1} \in \widehat{\mathcal{M}}\left(\phi_{1}\right)} \epsilon\left(u_{1}\right)\left(\prod_{i=1}^{\kappa} \lambda_{i}^{n_{z_{i}}\left(\phi_{1}\right)}\right),
$$


which is the coefficient of $\mathbf{y}$ in $\partial \mathbf{x}$ in $\operatorname{CF}(\Sigma, \boldsymbol{\alpha}, \boldsymbol{\beta}, \mathbf{z}, \mathfrak{s}) \otimes_{\mathbb{A}_{\tau}} \mathbb{A}_{\tau}\left[\lambda_{\kappa+1}\right]$.

With the same argument, the $C_{y}$-component of the differential of the generators in $C_{y}$ is identified with differential of $C_{y}=\mathrm{CF}(\Sigma, \boldsymbol{\alpha}, \boldsymbol{\beta}, \mathbf{z}, \mathfrak{s}) \otimes_{\mathbb{A}_{\tau}} \mathbb{A}_{\tau}\left[\lambda_{\kappa+1}\right]$.

We now consider the $C_{x}$-component of the differential of a generator in $C_{y}$. For any $\phi \in \pi_{2}(\mathbf{x} \times\{y\}, \mathbf{y} \times\{x\})$ we can write $\phi=\phi_{1} \# \phi_{2}$. By theorem 7.3 if $\mu(\phi)=1$ then $\mu\left(\phi_{1}\right)+\mu\left(\phi_{2}\right)-2 n_{v}\left(\phi_{2}\right)=1$. By Lipshitz' Index formula we have

$$
\mu\left(\phi_{2}\right)=2 n_{v}\left(\phi_{2}\right)+2 n_{z_{\kappa+1}}\left(\phi_{2}\right)+1 .
$$

This implies that $\mu\left(\phi_{2}\right)-2 n_{v}\left(\phi_{2}\right) \geq 1$, and that the equality holds if and only if $n_{z_{\kappa+1}}\left(\phi_{2}\right)=0$. Thus this last equality should be satisfied and $\mu\left(\phi_{1}\right)=0$. Hence $\phi_{1}$ is constant, $\mu\left(\phi_{2}\right)=1$ and $n_{v}\left(\phi_{2}\right)=0$. These conditions imply that the possible domains for $\phi_{2}$ are two different bi-gons in $S$ connecting $y$ to $x$, which contain $z_{\kappa}$ and $z_{\kappa+2}$ respectively. For either of these bi-gons $\widehat{\mathcal{M}}\left(\phi_{2}\right)$ consists of one element, while the orientation assignment for these two bi-gons is different. Using the relation $\widehat{f}\left(\lambda_{\kappa}\right)=\widehat{f}\left(\lambda_{\kappa+2}\right)$ in $\mathbb{A}\left[\lambda_{\kappa+1}\right]$, we may thus conclude that the coefficient of $\mathbf{y} \times\{x\}$ in $\partial(\mathbf{x} \times\{y\})$ is zero, i.e. the $C_{x}$-component of the differential of the generators in $C_{y}$ is trivial.

Finally, we consider the $C_{y}$-component of the differential of a generator in $C_{x}$. Again, degenerate $\phi \in \pi_{2}(\mathbf{x} \times\{x\}, \mathbf{y} \times\{y\})$ with $\mu(\phi)=1$ and $\lambda(\phi) \neq 0$ as the connected sum $\phi=\phi_{1} \# \phi_{2}$. We thus have $\mu\left(\phi_{1}\right)+\mu\left(\phi_{2}\right)-2 n_{v}\left(\phi_{2}\right)=1$, implying $\mu\left(\phi_{2}\right)-2 n_{v}\left(\phi_{2}\right) \leq 1$. By Lipshitz' index formula we have

$$
\mu\left(\phi_{2}\right)=2 n_{v}\left(\phi_{2}\right)+2 n_{z_{\kappa+1}}\left(\phi_{2}\right)-1,
$$

which implies that $\mu\left(\phi_{2}\right)$ is an odd number and $\mu\left(\phi_{2}\right)-2 n_{v}\left(\phi_{2}\right) \geq-1$. Thus $\mu\left(\phi_{2}\right)-2 n_{v}\left(\phi_{2}\right)$ is equal to 1 or -1 .

If $\mu\left(\phi_{2}\right)-2 n_{v}\left(\phi_{2}\right)=1$ then $\mu\left(\phi_{1}\right)=0$. Thus $\phi_{1}$ is constant and $\mathcal{D}(\phi)$ is the bigon containing $z_{\kappa+1}$. In this case $\# \widehat{\mathcal{M}}(\phi)=1$. Thus the corresponding component of the differential, as a map from $C_{x}$ to $C_{y}$, is given by

$$
\begin{aligned}
& \partial_{x y}^{1}: C_{x} \longrightarrow C_{y} \\
& \partial_{x y}^{1}(\mathbf{x} \times\{x\}):=\lambda_{\kappa+1}(\mathbf{x} \times\{y\}) .
\end{aligned}
$$

The second possibility is the case where $\mu\left(\phi_{2}\right)-2 n_{v}\left(\phi_{2}\right)=-1$. If $\mu\left(\phi_{2}\right)=$ $n_{v}\left(\phi_{2}\right)=1$ then $\mu\left(\phi_{1}\right)=2$ and $n_{w}\left(\phi_{1}\right)=1$. If furthermore $\mathfrak{R}(\tau)$ has at least one component with nonzero genus then by theorem 7.3 for sufficiently large connected sum length $\mathcal{M}(\phi)$ is identified with

$$
\begin{aligned}
\mathcal{M}\left(\phi_{1}\right) \times_{\mathbb{D}} \mathcal{M}\left(\phi_{2}\right) & =\left\{u_{1} \times u_{2} \in \mathcal{M}\left(\phi_{1}\right) \times \mathcal{M}\left(\phi_{2}\right) \mid \rho^{w}\left(u_{1}\right)=\rho^{v}\left(u_{2}\right)\right\} \\
& =\left\{u_{1} \times u_{2} \in \mathcal{M}\left(\phi_{1}\right) \times \mathcal{M}\left(\phi_{2}\right) \mid \rho^{w}\left(u_{1}\right)=u_{2}^{-1}(v)\right\} .
\end{aligned}
$$

Now $\mu\left(\phi_{2}\right)=n_{v}\left(\phi_{2}\right)=1$ implies that the domain of $\phi_{2}$ is the bi-gon in $S$ containing $v$ and thus it has a unique holomorphic representative up to translation. We can fix a holomorphic representative $u_{2}$ such that $u_{2}^{-1}(v)=(t, 0)$. Using an appropriate 
system of coherent orientations we thus have

$$
\begin{aligned}
\# \widehat{\mathcal{M}}(\phi) & =-\#\left\{u_{1} \in \mathcal{M}\left(\phi_{1}\right) \mid \rho^{w}\left(u_{1}\right)=(t, 0)\right\} \\
& =-\# \mathcal{M}\left(\phi_{1}, t\right)
\end{aligned}
$$

Let us now assume that the point $v$ is chosen very close to the curve $\beta$. By lemma 7.4 for $t$ sufficiently large $\mathcal{M}\left(\phi_{1}, t\right)$ is nonempty if and only if $\phi_{1} \in \pi_{2}^{\beta}(\mathbf{x})$ is the class of a $\beta$ boundary degeneration. If furthermore $l>1$ then $\# \mathcal{M}\left(\phi_{1}, t\right)=1$. Thus this case contributes to the $C_{y}$-component of the restriction of the differential to $C_{x}$ via a map

$$
\begin{aligned}
& \partial_{x y}^{2}: C_{x} \longrightarrow C_{y} \\
& \partial_{x y}^{2}(\mathbf{x} \times\{x\})=-\left(\prod_{\gamma_{\kappa} \neq \gamma_{i} \in \partial R_{l}^{+}} \lambda_{i}\right) \cdot(\mathbf{x} \times\{y\}) .
\end{aligned}
$$

Similarly, if $l=1$ then $\partial_{x y}^{2}(\mathbf{x} \times\{x\})=0$.

To deal with the other terms corresponding the homotopy classes $\phi$ with $n_{v}\left(\phi_{2}\right)>$ 1 we define a one parameter family of connected sum points $v(r)$ on $S$ such that when $r$ goes to infinity, $v(r)$ tends towards a point $v_{\infty}$ on the curve $\beta$.

Let $\mathcal{M}_{r}(\phi)$ be the moduli space of holomorphic representations of $\phi$ when we used the connected sum point $v(r)$ in $S$. Assume that for a sequence $\left\{r_{i}\right\}$ converges to infinity, the moduli space $\mathcal{M}_{r_{i}}(\phi) \neq \emptyset$ for all choice of connected sum length. For sufficiently large connected sum length the moduli space $\mathcal{M}_{r_{i}}(\phi)$ is identified with the fibered product $\mathcal{M}\left(\phi_{1}\right) \times_{\mathrm{Sym}^{k}(\mathbb{D})} \mathcal{M}\left(\phi_{2}\right)$. Consider a sequence $u_{1}^{i} \times u_{2}^{i}$ in the fibered product. Let $\bar{u}_{1}^{\infty}$ and $\bar{u}_{2}^{\infty}$ be Gromov limits of $\left\{u_{1}^{i}\right\}$ and $\left\{u_{2}^{i}\right\}$. The assumption $\mu\left(\phi_{1}\right)=2$ implies that there are three possible types for the limit $\bar{u}_{1}^{\infty}$. The limit can be a holomorphic disk or a singly broken flow line or it can contain a boundary degeneration. If it contain a boundary degeneration, $\lambda(\phi) \neq 0$ implies the remaining component has Maslov index zero and it should be constant. Thus $k=1$ and this situation is already considered in the previous case.

If $\bar{u}_{1}^{\infty}$ is not a broken flow line and it is a holomorphic disk, $\bar{u}_{2}^{\infty}$ has a component $u_{2}^{\infty}$ such that $\rho^{w}\left(\bar{u}_{1}^{\infty}\right)=\rho^{v}\left(u_{2}^{\infty}\right)$. Since $v\left(r_{i}\right)$ tends toward $v_{\infty}$ on $\beta, \rho^{v}\left(u_{2}^{\infty}\right)$ includes some points on $\{0\} \times \mathbb{R}$. Thus for large $i, \rho^{w}\left(u_{1}^{i}\right)$ contains points sufficiently close to $\{0\} \times \mathbb{R}$. By lemma 7.4 the holomorphic curve $u_{1}^{i}$ should be a boundary degeneration for $i$ sufficiently large. This implies that $k=1$ and again, we are within the cases considered earlier, and there is no new contribution to the $C_{y}$-component of the restriction of the differential to $C_{x}$ from this case.

Finally, if $\bar{u}_{1}^{\infty}$ is a broken flow line i.e. it is of the form $\bar{u}_{1}^{\infty}=a \star b$ and $\mu(a)=\mu(b)=1$, then $\bar{u}_{2}^{\infty}$ degenerates, correspondingly, as $\bar{u}_{2}^{\infty}=a^{\prime} \star b^{\prime}$. The Maslov index of $\phi_{2}$ is odd, thus one of $a^{\prime}$ and $b^{\prime}$ has odd Maslov index. Let us assume that $\mu\left(a^{\prime}\right)$ is odd. Then $\left(a^{\prime}\right)^{-1}\left(v^{\infty}\right)$ contains some points on $\{0\} \times \mathbb{R}$. If $a$ is the holomorphic representative of a homology class $\phi_{1}^{\prime}$ of a Whitney disk, then for $r$ sufficiently large $\mathcal{M}\left(\phi_{1}^{\prime}\right)$ includes holomorphic representatives $a^{r}$ such that $\rho^{w}\left(a^{r}\right)$ contains points of distance less than $1 / r$ to $\{0\} \times \mathbb{R}$. Since $\mu\left(\phi_{1}^{\prime}\right)=1, \phi_{1}^{\prime}$ has finitely 
many holomorphic representative up to translation. Thus for any holomorphic representative $u$ of $\phi_{1}^{\prime}, \rho^{w}(u)$ does not include points arbitrary close to $\{0\} \times \mathbb{R}$, since $w$ is not on $\boldsymbol{\beta}$.

Gathering the above considerations, we observe that if either of the two assumptions in the second part of theorem 7.3 is satisfied the $C_{y}$ component of the restriction of the differential to $C_{x}$ is given by the map

$$
\begin{aligned}
\partial_{x y} & : C_{x} \longrightarrow C_{y} \\
\partial_{x y}(\mathbf{x} \times\{x\}) & =\partial_{x y}^{1}(\mathbf{x} \times\{x\})+\partial_{x y}^{2}(\mathbf{x} \times\{x\}) \\
& =\lambda_{\kappa+1}(\mathbf{x} \times\{y\})-\left(\prod_{\gamma_{\kappa} \neq \gamma_{i} \in \partial R_{l}^{+}} \lambda_{i}\right) \cdot(\mathbf{x} \times\{y\}) \\
& =\left(\lambda_{\kappa+1}-\lambda\right)(\mathbf{x} \times\{y\}) .
\end{aligned}
$$

The proof in the case where all the components of $\mathfrak{R}(\tau)$ have genus zero and $\ell_{2}=g(\Sigma)$ is exactly the same as the last part of the proof of proposition 6.5 in OS8. This completes the proof of proposition 7.2 


\section{A TRIANGLE ASSOCIATED WITH SURGERY}

8.1. The triangle associated with the surgery Heegaard quadruple. Let us assume that

$$
H=\left(\Sigma, \boldsymbol{\alpha}=\left\{\alpha_{1}, \ldots, \alpha_{\ell}\right\}, \boldsymbol{\beta}=\left\{\beta_{1}, \ldots, \beta_{\ell}\right\}, \mathbf{z}_{0}=\left\{w_{1}, w_{2}, z_{3}, \ldots, z_{\kappa}\right\}\right)
$$

is a Heegaard diagram for the sutured manifold $(X, \tau)$ compatible with the surgery $(X, \tau) \rightsquigarrow\left(X, \tau_{\lambda}\right)$, where the two first marked points $w_{1}$ and $w_{2}$ correspond to the sutures $\gamma_{1}$ and $\gamma_{2}$ which are cut by the surgery simple closed curve $\lambda$. More precisely, assume that the marked points $w_{1}$ and $w_{2}$ are both placed very close to a point on $\beta_{\ell}$, such that $\beta_{\ell}$ separates them from each other. We will assume that

$$
\Sigma-\boldsymbol{\alpha}=\coprod_{i=1}^{k} A_{i}, \quad \& \quad \Sigma-\boldsymbol{\beta}=\coprod_{j=1}^{l} B_{j}
$$

are the connected components of $\Sigma-\boldsymbol{\alpha}$ and $\Sigma-\boldsymbol{\beta}$, respectively. We may assume that $A_{i}$ corresponds to $R_{i}^{-} \subset \mathfrak{R}^{-}(\tau)$ and that $B_{j}$ corresponds to $R_{j}^{+} \subset \mathfrak{R}^{+}(\tau)$ for $i=1, \ldots, k$ and $j=1, \ldots, l$. We will also assume that $w_{1}, w_{2} \in A_{1} \cap B_{1}$, that $A_{1}$ and $B_{1}$ are both surfaces of genus zero, and that the only marked points in $B_{1}$ are $w_{1}$ and $w_{2}$. All these assumptions may be achieved if $\gamma_{1}$ and $\gamma_{2}$ are on the boundary of $R_{1}^{-}$and $R_{1}^{+}$, where both these components have trivial genus and $R_{1}^{+}$is a cylinder (with $\gamma_{1}$ and $\gamma_{2}$ its boundary components). We will assume that $z_{i}$ corresponds to $\gamma_{i}$ for $3 \leq i \leq \kappa$. The surgery $(X, \tau) \rightsquigarrow\left(X, \tau_{\lambda}\right)$ is determined by a simple closed curve $\lambda$ on $\partial X$ which cuts $\gamma_{1}$ and $\gamma_{2}$ is a pair of transverse intersection points and remains disjoint from the curves in $\left\{\gamma_{3}, \ldots, \gamma_{\kappa}\right\}$. This curve determines a simple closed curve on $\Sigma$, still denoted by $\lambda$, which contains $w_{1}$ and $w_{2}$ as close-by points. We may assume that the only intersection point of this curve with the curves in $\boldsymbol{\beta}$ is at the point $p \in \beta_{\ell}$, located in the middle of the shorter arc on $\lambda$ with end points $w_{1}$ and $w_{2}$. Moreover, we may assume that $\lambda$ is completely included in the closure $\bar{A}_{1}$ of the sub-surface $A_{1}$ of $\Sigma$. Such a Heegaard diagram is called compatible with the surgery $(X, \tau) \rightsquigarrow\left(X, \tau_{\lambda}\right)$.

Suppose that $\lambda$ is as above. A neighborhood $N(\Lambda)$ of the union of curves

$$
\Lambda=\beta_{\ell} \cup \lambda \subset \Sigma
$$

may be identified with the complement of a very ball, or in fact a single point, in the standard torus $T=S^{1} \times S^{1}$, which will be denoted by $T^{\circ}$. The covering of $T$ by $\mathbb{R}^{2}$ gives a covering of $T^{\circ}$ by $\mathbb{R}^{2} \backslash \mathbb{Z}^{2}$. Any line with rational slope and not passing through the lattice points in $\mathbb{Z}^{2}$ gives a simple closed curve in $T^{\circ}$, and thus in the neighborhood $N(\Lambda)$ of $\Lambda$. If the slope of the line is $p / q \in \mathbb{Q}$, we may denote this simple closed curve by $\mu(p / q)$. Thus, $\mu(p / q)$ is homologous to $p \beta_{\ell}+q \lambda$. Any such simple closed curve is called a surgery curve. If $p / q$ is an integer, $\mu(p / q)$ determines a surgery on $(X, \tau)$ of the type discussed in section 2 More generally, for any rational number $p / q \in \mathbb{Q}$, the simple closed curve $\mu(p / q)$ determines a sutured manifold, which plays the role of Morse surgeries on knots inside closed three-manifolds. Such curves will be called surgery curves in short. 
Let us assume that $\mu_{0}, \mu_{1}$ and $\mu_{2}$ are three surgery curves for the Heegaard diagram $H$. Let us assume

$$
m_{0}=\#\left(\mu_{1} \cap \mu_{2}\right), \quad m_{1}=\#\left(\mu_{2} \cap \mu_{0}\right), \quad \& m_{2}=\#\left(\mu_{0} \cap \mu_{1}\right)
$$

are the algebraic intersection numbers of these curves. We will assume that the three curves (and the corresponding rational surgery coefficients) are chosen so that $m_{0}, m_{1}$ and $m_{2}$ are all positive integers. Let us assume

$$
\begin{gathered}
\mathbf{p}_{0}:=\mu_{1} \cap \mu_{2}=\left\{p_{0}=p_{0}^{1}, \ldots, p_{0}^{m_{0}}\right\}, \quad \mathbf{p}_{1}:=\mu_{2} \cap \mu_{0}=\left\{p_{1}=p_{1}^{1}, \ldots, p_{1}^{m_{1}}\right\}, \\
\& \mathbf{p}_{2}:=\mu_{0} \cap \mu_{1}=\left\{p_{2}=p_{2}^{1}, \ldots, p_{2}^{m_{2}}\right\} .
\end{gathered}
$$

We assume that the order of appearance of the points on the first curve in any of the above intersections is the same as the cyclic order determined by the indices. Furthermore, suppose that the three marked points $p_{0}, p_{1}$ and $p_{2}$ are the vertices of a very small triangle $\Delta$, which is one of the connected components in

$$
\Sigma-\boldsymbol{\alpha}-\left\{\beta_{1}, \ldots, \beta_{\ell-1}\right\}-\left\{\mu_{0}, \mu_{1}, \mu_{2}\right\} .
$$

We may assume that the intersection point $p$ is included in $\Delta$ as an interior marked point.

We may choose the marked points $z_{0}, z_{1}$ and $z_{2}$ outside $\Delta$ and very close to its edges, so that $z_{0}$ is close to the edge $e_{0}$ connecting $p_{1}$ to $p_{2}, z_{1}$ is close to the edge $e_{1}$ connecting $p_{2}$ to $p_{0}$, and $z_{2}$ is close to the edge $e_{2}$ connecting $p_{0}$ to $p_{1}$. The notation is illustrated in figure 3 . We will denote by $\mathbf{z}$ the following set of marked points

$$
\mathbf{z}=\left\{z_{0}, z_{1}, \ldots, z_{\kappa}, p\right\}
$$

Consider the Heegaard diagrams

$$
H_{i}=\left(\Sigma, \boldsymbol{\alpha}, \boldsymbol{\beta}^{i}=\left\{\beta_{1}^{i}, \ldots, \beta_{\ell-1}^{i}, \mu_{i}\right\}, \mathbf{z}\right), \quad i \in\{0,1,2\},
$$

where we assume that $\beta_{j}^{i}$ are small Hamiltonian isotopes of the curve $\beta_{j}$, for $i=$ $0,1,2$, so that any pair of curves in $\left\{\beta_{j}^{0}, \beta_{j}^{1}, \beta_{j}^{2}\right\}$ intersect each-other in a pair of transverse canceling intersection points for $j=1, \ldots, \ell-1$. We would like to study the Heegaard quadruple (with $\kappa+2$ marked points)

$$
R=\left(\Sigma, \boldsymbol{\alpha}, \boldsymbol{\beta}^{0}, \boldsymbol{\beta}^{1}, \boldsymbol{\beta}^{2}, \mathbf{z}\right)
$$

in this section and construct a triangle of chain complexes associated with it, which generalizes the exact triangles associated with surgery on knots in the context of Heegaard Floer theory of closed three-manifolds and knots inside them.

The Heegaard diagram $\left(\Sigma, \boldsymbol{\alpha}, \boldsymbol{\beta}^{i}, \mathbf{z}\right)$, for $i=0,1,2$ determines a sutured manifold which will be denoted by $\left(Y, \varsigma^{i}\right)$. In fact, instead of gluing a disk to $\mu_{i}$, one may fill out the suture $\gamma_{p}$ corresponding to the marked point $p$ and obtain the same three manifold $Y$. The identification is illustrated in figure 4. Thus the three-manifold $Y$ does not depend on $i$, while the sutured manifold structure $\varsigma^{i}$ is determined by $i$. The above identification of the three manifold $Y$ gives an identification of the spaces of relative $\operatorname{Spin}^{c}$ structures as well:

$$
\operatorname{Spin}^{c}(Y, \varsigma):=\operatorname{Spin}^{c}\left(Y, \varsigma^{0}\right)=\operatorname{Spin}^{c}\left(Y, \varsigma^{1}\right)=\operatorname{Spin}^{c}\left(Y, \varsigma^{2}\right) .
$$




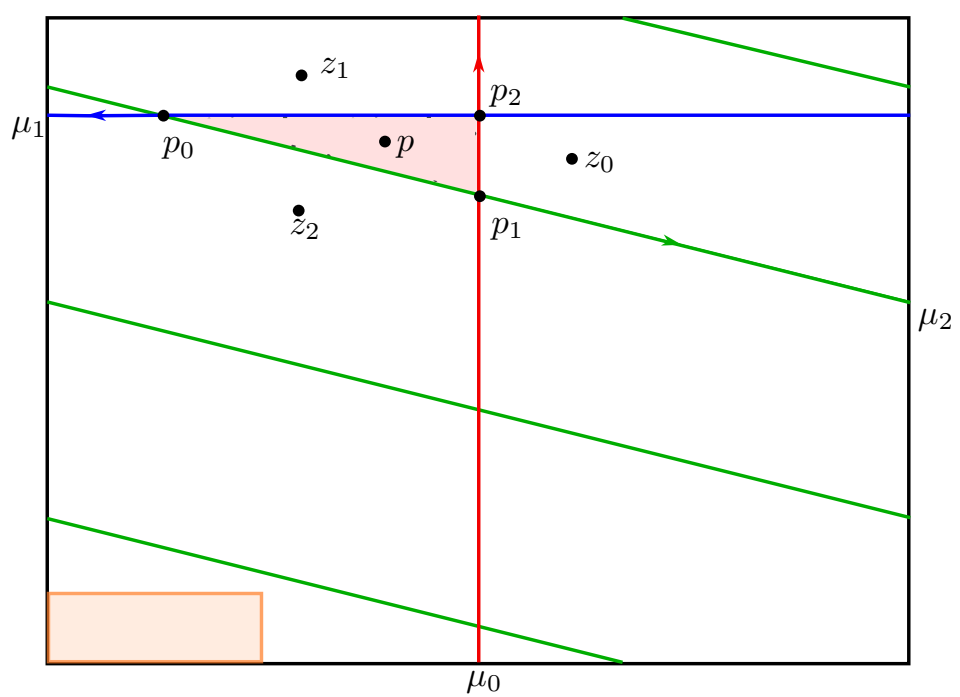

Figure 3. A neighborhood of the curves $\mu_{0}, \mu_{1}$ and $\mu_{2}$ is illustrated. One should take the connected sum of the torus obtained by identifying the opposite edges of the rectangle, with another Riemann surface to obtain the Heegaard surface $\Sigma$. The attaching circle of the connected sum tube lands in the shaded area in the lower left corner of the figure. The $\alpha$ curves live close to the boundary of the rectangle, or on the Riemann surface which is attached to this torus. The marked points $\left\{p, z_{0}, z_{1}, z_{2}\right\}$ and the intersection points $p_{0}, p_{1}$ and $p_{2}$ are illustrated. For this picture $m_{0}=m_{2}=1$ while $m_{1}=3$.

Note that $\operatorname{Spin}^{c}(Y, \varsigma)$ is just a notation we use for this common space of relative $\operatorname{Spin}^{c}$ structures, and that the identification of the $\operatorname{Spin}^{c}$ spaces is not natural.

For $i$ a cyclic index in $\frac{\mathbb{Z}}{3 \mathbb{Z}}=\{0,1,2\}$, let $\left(X, \tau^{i}\right)$ be the sutured manifold obtained from $\left(Y, \varsigma^{i}\right)$ by filling out the sutures corresponding to $z_{i+1}$ and $z_{i+2}$ (note that we are taking indices modulo 3 ). The intersection points $p_{i} \in \mu_{i+1} \cap \mu_{i+2}$, for $i \in \frac{\mathbb{Z}}{3 \mathbb{Z}}=\{0,1,2\}$ determine an identification of three subsets

$$
\overline{\mathfrak{S}}_{i} \subset \operatorname{Spin}^{c}\left(\bar{Y}^{\varsigma^{i}}\right)=\operatorname{Spin}^{c}\left(\bar{X}^{\tau^{i}}\right), \quad i \in\{0,1,2\} .
$$

Let us denote these identified subsets by $\operatorname{Spin}^{c}(\bar{X})$. Again in a sense, we are abusing the language with this notation, at least when $\lambda$ is not null-homologous. We will fix a class $\mathfrak{s} \in \operatorname{Spin}^{c}(\bar{X})$ for the rest of this section. We will assume that the Heegaard diagrams $H_{i}, i=0,1,2$ are $\mathfrak{s}$-admissible. When $\lambda$ is null-homologous this is guaranteed if the Heegaard diagram $H$ is $\mathfrak{s}$-admissible. In fact, we will drop the admissibility issues, as well as orientation issues, from our discussion in the remainder of this section. Taking care of these issues is completely straight forward, and follows the lines of the arguments given in the earlier sections. 
A.
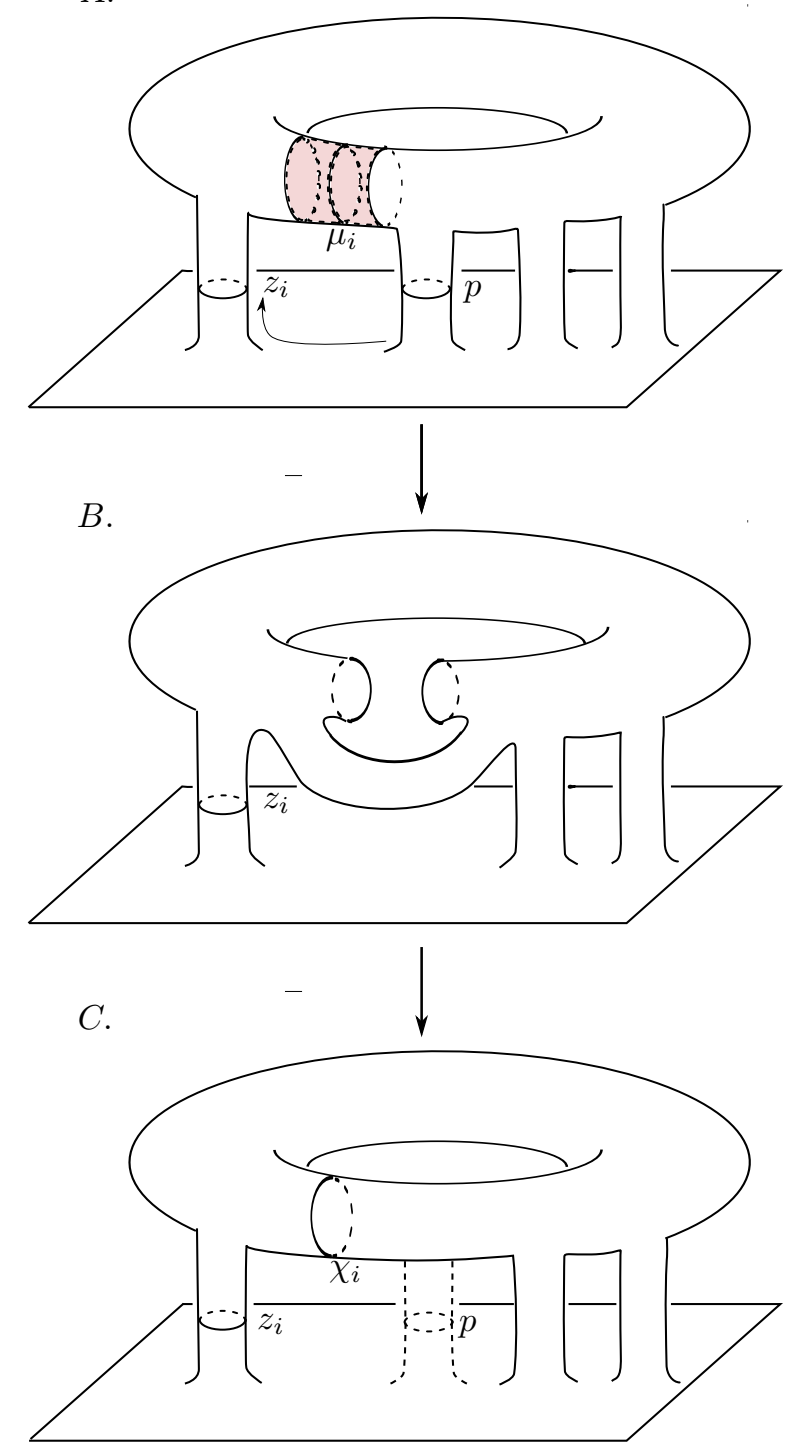

FiguRE 4. Instead of attaching a 2-handle along the curve $\mu_{i}$, one may fill in the suture which corresponds to the marked point $p$. In part A of the figure, a $2=$ handle is attached to $\mu_{i}$ (think of $X$ as the three manifold outside the torus and above the plane illustrated in this picture). Then we may slide the 1-handle corresponding to $p$ over the 1-handle corresponding to $z_{i}$, as illustrated in part B. The result, after smoothing the appropriate corners, is the picture illustrated in part $\mathrm{C}$, in which the suture corresponding to $p$ is filled out and instead, no 2-handle is attached to $\mu_{i}$. 
The algebra $\mathbb{A}_{\varsigma^{i}}$ is independent of $i$, and will be denoted by $\widehat{\mathbb{A}}$. Let us denote the generator corresponding to the marked point $z_{j}$ by $\lambda_{j}$, for $j=0,1, \ldots, \kappa$. The generator associated with the marked point $p$ will be denoted by $\lambda_{p}$. We would like to consider the following quotient of $\widehat{\mathbb{A}}$ :

$$
\mathbb{A}=\frac{\widehat{\mathbb{A}}}{\left\langle\lambda_{p}-\left(\lambda_{0}^{m_{0}-1} \lambda_{1}^{m_{1}-1} \lambda_{2}^{m_{2}-1}\right)\right\rangle_{\widehat{\mathbb{A}}}} .
$$

Furthermore, let us denote the generator associated with the marked point $z_{j}$ in $\mathbb{A}_{\tau^{i}}$ by $\zeta_{j}$ for $j=3, \ldots, \kappa$, and denote the generator associated with $w_{1}$ and $w_{2}$ by $\zeta_{1}$ and $\zeta_{2}$ respectively. Note that $\mathbb{A}_{i}=\mathbb{A}_{\tau^{i}}$ does not depend on $i \in\{0,1,2\}$, either. Each Heegaard diagram $H_{i}$ determines an embedding of $\mathbb{A}_{i}$ in $\mathbb{A}$. More precisely, we may define

$$
\imath^{i}: \mathbb{A}_{i} \rightarrow \mathbb{A}, \quad \imath^{i}\left(\zeta_{j}\right)=\left\{\begin{array}{ll}
\lambda_{i} & \text { if } j=1 \\
\frac{\lambda_{p} \lambda_{0} \lambda_{1} \lambda_{2}}{\lambda_{i}} & \text { if } j=2 \\
\lambda_{j} & \text { if } 3 \leq j \leq \kappa
\end{array} .\right.
$$

As mentioned earlier, $\mathbb{A}_{0}, \mathbb{A}_{1}$ and $\mathbb{A}_{2}$ are isomorphic. However, the index is used to distinguish them as sub-rings of $\mathbb{A}$, using the embedding $\imath^{i}: \mathbb{A}_{i} \rightarrow \mathbb{A}$.

Let $\boldsymbol{\beta}_{0}$ denote the set of curves $\left\{\beta_{1}, \ldots, \beta_{\ell-1}\right\}, \mathbb{D}_{\alpha}$ denote a set of $\ell$ copies of $D^{2} \times[-\epsilon, \epsilon]$ (for some small positive real number $\epsilon$ ) corresponding to the curves in $\boldsymbol{\alpha}$ and $\mathbb{D}_{\beta_{0}}$ denote a set of $\ell-1$ copies of $D^{2} \times[-\epsilon, \epsilon]$ corresponding to curves in $\boldsymbol{\beta}_{0}$. Denote small tubular neighborhoods of the curves in $\boldsymbol{\alpha}$ and the curves in $\boldsymbol{\beta}_{0}$ by $\operatorname{nd}(\boldsymbol{\alpha})$ and $\operatorname{nd}\left(\boldsymbol{\beta}_{0}\right)$, respectively. These neighborhoods may be identified with subsets of $\partial \mathbb{D}_{\alpha}$ and $\partial \mathbb{D}_{\beta_{0}}$ respectively. Under the identification of $Y$ with the three-manifold

$$
([0,1] \times \Sigma \backslash(\mathbf{z}-\{p\})) \underset{\operatorname{nd}(\alpha) \times\{0\}}{\bigcup} \mathbb{D}_{\alpha} \bigcup_{\operatorname{nd}\left(\beta_{0}\right) \times\{1\}} \mathbb{D}_{\beta_{0}},
$$

each marked point $z_{j}$ determines an oriented simple closed curve on the boundary of $Y$. The Poincaré dual of this curve determines an element $\chi_{j} \in \mathrm{H}^{2}(Y, \partial Y ; \mathbb{Z})$ for $3 \leq j \leq \ell$. For $i \in\{0,1,2\}$ we will denote the element of $\mathrm{H}^{2}(Y, \partial Y ; \mathbb{Z})$ corresponding to the marked point $z_{i}$ by $\eta_{i}$. The assumptions on the Heegaard diagram imply that

$$
\eta_{0}+\eta_{1}+\eta_{2}=0
$$

The Poincaré duals of the curves corresponding to the marked point $z_{j}$ and $p$ in $\left(Y, \varsigma^{i}\right)$ will be denoted by $\chi(i, j)$ and $\chi(i, p)$ respectively, for $i=0,1,2$ and $0 \leq j \leq \ell$. Furthermore, let $\chi_{i}$ denote the Poincaré dual $\mathrm{PD}\left[\mu_{i}\right]$ of the simple closed curve $\mu_{i} \subset \partial Y$ for $i=0,1,2$. One may check that

$$
\begin{aligned}
& \chi(i, j)= \begin{cases}\chi_{j} & \text { if } j \neq i \& j \in\{3, \ldots, \kappa\} \\
\eta_{j} & \text { if } j \neq i \& j \in\{0,1,2\} \\
\chi_{i}+\eta_{i} & \text { if } j=i\end{cases} \\
& \chi(i, p)=-\chi_{i} .
\end{aligned}
$$


Associated with any of the Heegaard diagrams $H_{i}, i=0,1,2$ (and independent of $i$ ) we define a filtration map

$$
\begin{aligned}
& \chi: G(\mathbb{A}) \longrightarrow \mathrm{H}^{2}(Y, \partial Y ; \mathbb{Z}), \\
& \chi\left(\lambda_{j}\right):= \begin{cases}\chi_{j} & \text { if } j \in\{0,1, \ldots, \kappa\} . \\
-\left(\chi_{0}+\chi_{1}+\chi_{2}\right) & \text { if } j=p\end{cases}
\end{aligned}
$$

Note that with this assignment we may compute

$$
\chi \circ \imath^{i}\left(\prod_{j=1}^{\kappa} \zeta_{j}^{i_{j}}\right)=\left(i_{1}-i_{2}\right) \chi_{i}+\sum_{j=3}^{\kappa} i_{j} \chi_{j}, \quad \forall i \in\{0,1,2\} .
$$

This implies that the filtration $\chi: G(\mathbb{A}) \rightarrow \mathrm{H}^{2}(Y, \partial Y ; \mathbb{Z})$ is compatible with the filtration of $G\left(\mathbb{A}_{i}\right)$ by $\mathrm{H}^{2}(X, \partial X ; \mathbb{Z})$.

Let us consider the following quotient of $\operatorname{Spin}^{c}(Y, \varsigma)$ :

$$
\mathfrak{S}=\operatorname{Spin}^{c}(X, \tau):=\frac{\operatorname{Spin}^{c}(Y, \varsigma)}{\left\langle\eta_{0}, \eta_{1}, \eta_{2}\right\rangle_{\mathbb{Z}}}=\operatorname{Spin}^{c}(X, \tau) .
$$

The last equality follows since $\operatorname{Spin}^{c}\left(X, \tau^{i}\right)$ is obtained from $\operatorname{Spin}^{c}\left(Y, \varsigma^{i}\right)$ by setting trivial the sutures corresponding to $z_{j}$ with $j \in\{0,1,2\}-\{i\}$. Thus, it is equal to the quotient of $\operatorname{Spin}^{c}\left(Y, \varsigma^{i}\right)$ by the action of $\eta_{j}$, with $j \in\{0,1,2\}-\{i\}$. Since we have $\eta_{0}+\eta_{1}+\eta_{2}=0$, this means that $\eta_{0}, \eta_{1}$, and $\eta_{2}$ act trivially on $\operatorname{Spin}^{c}\left(X, \tau^{i}\right)$.

Correspondingly, consider the following $\mathbb{Z}$ module associated with the threemanifold $Y$ :

$$
\mathbb{H}:=\frac{\mathrm{H}^{2}(Y, \partial Y ; \mathbb{Z})}{\left\langle\eta_{0}, \eta_{1}, \eta_{2}\right\rangle_{\mathbb{Z}}}=\mathrm{H}^{2}(X, \partial X ; \mathbb{Z})
$$

We continue to denote the image of $\chi_{i} \in \mathrm{H}^{2}(Y, \partial Y ; \mathbb{Z})$ in $\mathbb{H}$ by $\chi_{i}$. Clearly, $\mathbb{H}$ acts on $\mathfrak{S}$. From the definition, we also have a natural quotient map

$$
\operatorname{Spin}^{c}(Y, \varsigma) \longrightarrow \operatorname{Spin}^{c}(X, \tau)=\mathfrak{S}
$$

which will be denoted by (.) (thus, this map sends a relative $\operatorname{Spin}^{c}$ class $\mathfrak{s}$ to its class $(\underline{\mathfrak{s}}) \in \mathfrak{S}$.

The filtration map $\chi: G(\mathbb{A}) \rightarrow \mathrm{H}^{2}(Y, \partial Y ; \mathbb{Z})$ may be composed with the quotient map from $\mathrm{H}^{2}(Y, \partial Y ; \mathbb{Z})$ to $\mathbb{H}$ to define a new filtration map, yet denoted by $\chi$.

For $i=0,1,2$, consider the filtered $(\mathbb{A}, \mathbb{H})$ chain complex

$$
\begin{aligned}
\mathbb{E}_{i}(\mathfrak{s}): & =\mathrm{CF}\left(Y, \varsigma^{i}\right) \otimes_{\widehat{\mathbb{A}}} \mathbb{A}=\mathrm{CF}\left(X, \tau^{i}, \mathfrak{s}\right) \otimes_{\mathbb{A}_{\tau} i} \mathbb{A} \\
& =\left\langle\mathbf{x} \mid \mathbf{x} \in \mathbb{T}_{\alpha} \cap \mathbb{T}_{\beta^{i}}, \quad \& \underline{\mathfrak{s}}_{\mathbf{z}}(\mathbf{x}) \in \mathfrak{s}\right\rangle_{\mathbb{A}}
\end{aligned}
$$

The set of marked points $\mathbf{z}=\left\{z_{0}, \ldots, z_{\kappa}, p\right\}$ defines a map

$$
\begin{aligned}
& \lambda_{\mathbf{z}}: \coprod_{i=0}^{3} \coprod_{\mathbf{x}, \mathbf{y} \in \mathbb{T}_{\alpha} \cap \mathbb{T}_{\beta^{i}}} \pi_{2}(\mathbf{x}, \mathbf{y}) \longrightarrow G(\mathbb{A}) \\
& \lambda_{\mathbf{z}}(\phi):=\lambda_{p}^{n_{p}(\phi)} \cdot \prod_{i=0}^{\kappa} \lambda_{i}^{n_{i}(\phi)}=\lambda_{p}^{n_{p}(\phi)} \lambda_{0}^{n_{z_{0}}(\phi)} \lambda_{1}^{n_{z_{1}}(\phi)} \ldots \lambda_{\kappa}^{n_{z_{\kappa}}(\phi)} .
\end{aligned}
$$


The differential $\partial_{i}$ of the complex $\mathbb{E}_{i}(\mathfrak{s})$, as an $\mathbb{A}$-module homomorphism, is defined by

$$
\partial_{i}(\mathbf{x}):=\sum_{\mathbf{y} \in \mathbb{T}_{\alpha} \cap \mathbb{T}_{\beta^{i}}} \sum_{\phi \in \pi_{2}^{1}(\mathbf{x}, \mathbf{y})}\left(\mathfrak{m}(\phi) \lambda_{\mathbf{z}}(\phi)\right) \mathbf{y}
$$

Here $n_{i}(\phi)$ denotes the intersection number $n_{z_{i}}(\phi)$, and $\pi_{2}^{1}(\mathbf{x}, \mathbf{y}) \subset \pi_{2}(\mathbf{x}, \mathbf{y})$ consists of all homotopy types $\phi$ of Whitney disks connecting $\mathbf{x}$ to $\mathbf{y}$ such that $\mu(\phi)=1$. We define a map from the set of generators of $\mathbb{E}_{i}(\mathfrak{s})$ to $\mathfrak{S}$ by setting

$$
\begin{aligned}
& \underline{\mathfrak{s}}^{i}: G(\mathbb{A}) \times\left(\mathbb{T}_{\alpha} \cap \mathbb{T}_{\beta^{i}}\right) \longrightarrow \mathfrak{S} \\
& \underline{\mathfrak{s}}^{i}(\lambda \mathbf{x}):=(\underline{\mathfrak{s}}(\mathbf{x}))+\chi(\lambda) .
\end{aligned}
$$

Abusing the notation, we will sometimes denote $\underline{\mathfrak{s}}^{i}(\lambda \mathbf{x})$ by $\underline{\mathfrak{s}}(\lambda \mathbf{x})=\underline{\mathfrak{s}}(\mathbf{x})+\chi(\lambda)$, dropping the index $i$ and the quotient map (.) $: \operatorname{Spin}^{c}(Y, \varsigma) \rightarrow \mathfrak{S}$ from the notation.

Lemma 8.1. If a generator $\lambda \mathbf{y}$, with $\lambda \in G(\mathbb{A})$, appears with non-zero coefficient in $\partial_{i}(\mathbf{x})$, we will have $\underline{\mathfrak{s}}^{i}(\mathbf{x})=\underline{\mathfrak{s}}^{i}(\lambda \mathbf{y})$ in $\mathfrak{S}$.

Proof. Without loosing on generality, let us assume that $i=0$. Suppose that $\underline{\mathfrak{s}}(\mathbf{x}), \underline{\mathfrak{s}}(\mathbf{y}) \in \mathfrak{s}$, and that there is a Whitney disk $\phi \in \pi_{2}(\mathbf{x}, \mathbf{y})$ contributing to $\partial_{0}(\mathbf{x})$ with $\lambda=\lambda_{\mathbf{z}}(\phi)$. Then we will have $n_{1}(\phi)=n_{2}(\phi)=n_{p}(\phi)$. The existence of this disk implies that

$$
\begin{aligned}
\underline{\mathfrak{s}}(\mathbf{x}) & =\underline{\mathfrak{s}}(\mathbf{y})+\left(n_{0}(\phi)-n_{p}(\phi)\right) \chi_{0}+\sum_{j=3}^{\kappa} n_{j}(\phi) \cdot \chi_{j} \\
\Rightarrow \quad \underline{\mathfrak{s}}^{0}(\mathbf{x}) & =\underline{\mathfrak{s}}^{0}(\mathbf{y})+\chi\left(\lambda_{p}^{n_{p}(\phi)} \prod_{j=0}^{\kappa} \lambda_{j}^{n_{j}(\phi)}\right)=\underline{\mathfrak{s}}^{0}(\lambda \mathbf{y}) .
\end{aligned}
$$

For the equality in the second line, we use the equality $\chi\left(\lambda_{p}\right)=-\left(\chi_{0}+\chi_{1}+\chi_{2}\right)$. This completes the proof of the lemma.

The above assignment of relative $\operatorname{Spin}^{c}$ structures is thus respected by the differential $\partial_{i}$ of $\mathbb{E}_{i}(\mathfrak{s})$, and $\mathbb{E}_{i}(\mathfrak{s})$ is thus decomposed as

$$
\mathbb{E}_{i}(\mathfrak{s})=\bigoplus_{\mathfrak{s} \in \mathfrak{s} \subset \mathfrak{S}} \mathbb{E}_{i}(\underline{\mathfrak{s}})
$$

Associated with the $\operatorname{Spin}^{c}$ class $\mathfrak{s}$, we will describe a triangle of chain maps

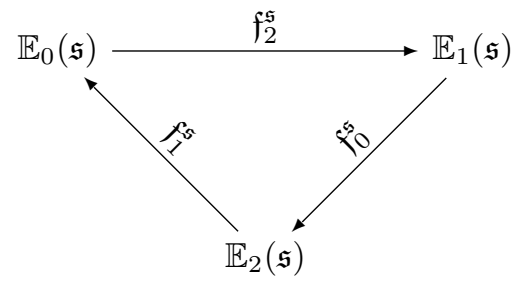

such that the compositions $\mathfrak{f}_{i+1}^{\mathfrak{s}} \circ \mathfrak{f}_{i}^{\mathfrak{s}}, i \in \frac{\mathbb{Z}}{3 \mathbb{Z}}=\{0,1,2\}$ are chain homotopic to zero. 
To define the chain map $\mathfrak{f}_{i-1}^{\mathfrak{s}}$, note that the $\operatorname{special} \operatorname{Heegaard} \operatorname{diagram}\left(\Sigma, \boldsymbol{\beta}^{i}, \boldsymbol{\beta}^{i+1}, \mathbf{z}\right)$ is admissible for all the corresponding $\operatorname{Spin}^{c}$ classes (c.f. the arguments of subsection 6.2. We may thus compute

$$
\mathrm{CF}\left(L_{i-1}, \nu_{i-1} ; \mathbb{A}\right)=\mathrm{CF}\left(\Sigma, \boldsymbol{\beta}^{i}, \boldsymbol{\beta}^{i+1}, \mathbf{z}\right) \otimes \mathbb{A}
$$

where $\left(L_{i-1}, \nu_{i-1}\right)$ is the sutured manifold corresponding to the special Heegaard diagram $\left(\Sigma, \boldsymbol{\beta}^{i}, \boldsymbol{\beta}^{i+1}, \mathbf{z}\right)$. The intersection point $p_{i-1} \in \mu_{i} \cap \mu_{i+1}$ determines a unique $\operatorname{Spin}^{c}$ class

$$
\mathfrak{s}_{i-1} \in \operatorname{Spin}^{c}\left(\overline{L_{i-1}}\right), \quad c_{1}\left(\mathfrak{s}_{i-1}\right)=0
$$

as well as a top generator $\Theta_{i-1}$ corresponding to $\mathfrak{s}_{i-1}$ (which is a closed element) in the above Heegaard Floer complex. The generator $\Theta_{i-1}$ is obtained as the union of $p_{i-1}$ and the positive intersection points of $\beta_{j}^{i}$ and $\beta_{j}^{i+1}$ for $j=1, \ldots, \ell-1$. The generator $\Theta_{i-1}$ corresponds to a relative $\operatorname{Spin}^{c}$ class which will be denoted by $\underline{\mathfrak{s}}_{i-1} \in \mathfrak{s}_{i-1}$. Consider the holomorphic triangle map

$$
f_{i-1}^{\mathfrak{s}}: \mathbb{E}_{i}(\mathfrak{s}) \otimes_{\mathbb{A}} \mathrm{CF}\left(\Sigma, \boldsymbol{\beta}^{i}, \boldsymbol{\beta}^{i+1}, \mathbf{z} ; \mathfrak{s}_{i-1} ; \mathbb{A}\right) \longrightarrow \mathbb{E}_{i+1}(\mathfrak{s}) .
$$

On a generator $\mathbf{x} \otimes \mathbf{q}$ of the left hand side, with $\mathbf{x} \in \mathbb{T}_{\alpha} \cap \mathbb{T}_{\beta^{i}}$ and $\mathbf{q}$ a generator corresponding to the $\operatorname{Spin}^{c}$ class $\mathfrak{s}_{i-1}, f_{i-1}^{\mathfrak{s}}(\mathbf{x} \otimes \mathbf{q})$ is defined by

$$
f_{i-1}^{\mathfrak{s}}(\mathbf{x} \otimes \mathbf{q}):=\sum_{\mathbf{y} \in \mathbb{T}_{\alpha} \cap \mathbb{T}_{\beta}} \sum_{\Delta \in \pi_{2}^{0}(\mathbf{x}, \mathbf{q}, \mathbf{y})}\left(\mathfrak{m}(\Delta) \lambda_{\mathbf{z}}(\Delta)\right) \mathbf{y},
$$

where $\pi_{2}^{0}(\mathbf{x}, \mathbf{q}, \mathbf{y})$ denotes the subset of $\pi_{2}(\mathbf{x}, \mathbf{q}, \mathbf{y})$ consisting of the triangle classes $\Delta$ such that $\mu(\Delta)=0$. The map $f_{i-1}^{\mathfrak{s}}$ is then extended, as an $\mathbb{A}$-module homomorphism, to all of $\mathbb{E}_{i}(\mathfrak{s}) \otimes_{\mathbb{A}} \mathrm{CF}\left(\Sigma, \boldsymbol{\beta}^{i}, \boldsymbol{\beta}^{i+1}, \mathbf{z} ; \mathfrak{s}_{i-1} ; \mathbb{A}\right)$.

One should also fix the $\operatorname{Spin}^{c}$ class of the triangles contributing to the sum in equation 17. Let us assume that the intersection points $\mathbf{x}_{i} \in \mathbb{T}_{\alpha} \cap \mathbb{T}_{\beta^{i}}$ for $i=0,1,2$ are fixed so that $\underline{\mathfrak{s}}^{i}(\mathbf{x}) \in \mathfrak{s} \subset \mathfrak{S}$. Furthermore, assume that, after possible re-labeling of the curves in $\boldsymbol{\alpha}$, we have

$$
\mathbf{x}_{i}=\left\{x_{i}^{1}, \ldots, x_{i}^{\ell}\right\}, \quad x_{i}^{j} \in\left\{\begin{array}{ll}
\alpha_{j} \cap \beta_{j}^{i} & \text { if } 1 \leq j<\ell \\
\alpha_{\ell} \cap \mu_{i} & \text { if } j=\ell
\end{array} .\right.
$$

Also, for $j=1, \ldots, \ell-1$, we will assume that $x_{0}^{j}, x_{1}^{j}$ and $x_{2}^{j}$ are very close to each other, and correspond to one another by the Hamiltonian isotopies considered above. We may always change the $\alpha$ curves in the Heegaard diagram by isotopy so that the above condition is satisfied. In order to specify the class of triangles used in equation 17 , we need to specify triangle classes $\Delta_{i} \in \pi_{2}\left(\mathbf{x}_{i+1}, \Theta_{i}, \mathbf{x}_{i-1}\right)$ for any $i \in \frac{\mathbb{Z}}{3 \mathbb{Z}}=\{0,1,2\}$. The domain $\mathcal{D}\left(\Delta_{i}\right)$ consists of a union of $\ell$ triangles. The first $\ell-1$ triangles are small triangles determined by the small Hamiltonian isotopy changing the simple closed curves in $\boldsymbol{\beta}^{i+1}-\left\{\mu_{i+1}\right\}$ to those in $\boldsymbol{\beta}^{i-1}-\left\{\mu_{i-1}\right\}$. Two of the vertices of the $j$-th triangle are the intersection points $x_{i+1}^{j}$ and $x_{i-1}^{j}$, while the last vertex belongs to the top generator $\Theta_{i}$. The $\ell$-th triangle connects three intersection points between $\mu_{i}, \mu_{i+1}$ and $\alpha_{\ell} \in \boldsymbol{\alpha}$. With this notation fixed, let

$$
\mathcal{D}=\mathcal{D}\left(\Delta_{0}\right)+\mathcal{D}\left(\Delta_{1}\right)+\mathcal{D}\left(\Delta_{2}\right)
$$

We assume that no $\alpha$ curve appears in $\partial \mathcal{D}$. Furthermore, we may assume that $n_{p}(\mathcal{D})=-1$ while $n_{j}(\mathcal{D})=0$ for $j=0,1, \ldots, \kappa$. $\mathcal{D}$ is then the domain of a triangle class $\widehat{\Delta} \in \pi_{2}\left(\Theta_{0}, \Theta_{1}, \Theta_{2}\right)$ with small area. Note that achieving all these properties 
may be done through a correct choice of the last triangle among the $\ell$ triangles chosen above.

The choice of this last triangle class (with the above properties) determines how the map $f_{i-1}^{\mathfrak{s}}$ changes the relative $\operatorname{Spin}^{c}$ classes. We will specify this last choice after the following lemma.

Lemma 8.2. There exists a cohomology class $h_{i} \in \mathbb{H}$ for $i=0,1,2$ with the following property. If for a generator $\mathbf{x}$ of $\mathbb{E}_{i}(\mathfrak{s})$ we have

$$
\underline{\mathfrak{s}}(\mathbf{x})=\underline{\mathfrak{s}} \in \mathfrak{s} \subset \mathfrak{S},
$$

and for the intersection point $\mathbf{q} \in \mathbb{T}_{\beta^{i}} \cap \mathbb{T}_{\beta^{i+1}}$ we have $\underline{\mathfrak{s}}(\mathbf{q})=\underline{\mathfrak{s}}_{i-1}$, then

$$
f_{i-1}^{\mathfrak{s}}(\mathbf{x} \otimes \mathbf{q}) \in \mathbb{E}_{i+1}\left(\underline{\mathfrak{s}}+h_{i-1}\right)
$$

Furthermore, the cohomology classes satisfy

$$
h_{0}+h_{1}+h_{2}=-\left(\chi_{0}+\chi_{1}+\chi_{2}\right) \text {. }
$$

Proof. Once again, it suffices to prove the lemma for $i=0$. The cyclic symmetry of all definitions then implies the lemma in general. Let $\mathbf{q} \in \mathbb{T}_{\beta^{0}} \cap \mathbb{T}_{\beta^{1}}$ be an intersection point corresponding to the relative Spin ${ }^{c}$ class $\underline{\mathfrak{g}}_{2}$. Suppose that $\mathbf{y} \in$ $\mathbb{T}_{\alpha} \cap \mathbb{T}_{\beta^{1}}$ is a generator such that $\pi_{2}(\mathbf{x}, \mathbf{q}, \mathbf{y})$ is non-empty, and that $\Delta$ is a triangle class in this set. Then, using the fact that $n_{2}(\phi)=n_{p}(\phi)$ we will have

$$
\begin{aligned}
\underline{\mathfrak{s}}^{0}(\mathbf{x}) & =\left(\underline{\mathfrak{s}}(\mathbf{y})+h_{2}\right)+\left(n_{0}(\phi)-n_{p}(\phi)\right) \chi_{0}+\left(n_{1}(\phi)-n_{p}(\phi)\right) \chi_{1}+\sum_{j=3}^{\kappa} n_{j}(\phi) \chi_{j} \\
& =\left(\underline{\mathfrak{s}}^{1}(\mathbf{y})+h_{2}\right)-n_{p}(\phi)\left(\chi_{0}+\chi_{1}+\chi_{2}\right)+\sum_{j=0}^{\kappa} n_{j}(\phi) \chi_{j} \\
& =\underline{\mathfrak{s}}^{1}\left(\lambda_{\mathbf{z}}(\phi) \cdot \mathbf{y}\right)+h_{2} .
\end{aligned}
$$

Here $h_{2}$ is a cohomology class in $\mathbb{H}$ which depends on our identification of spaces of relative $\operatorname{Spin}^{c}$ classes associated with the diagram, and is chosen once for all. Consider triangle classes

$$
\Delta_{i-1} \in \pi_{2}\left(\mathbf{x}_{i}, \Theta_{i-1}, \mathbf{x}_{i+1}\right), \quad i \in\{0,1,3\}=\frac{\mathbb{Z}}{3 \mathbb{Z}}
$$

corresponding to the Heegaard diagrams $\left(\Sigma, \boldsymbol{\alpha}, \boldsymbol{\beta}^{i}, \boldsymbol{\beta}^{i+1}, \mathbf{z}\right)$ defined earlier. We have assumed that the triangle classes are chosen so that

$$
\mathcal{D}=\mathcal{D}\left(\Delta_{0}\right)+\mathcal{D}\left(\Delta_{1}\right)+\mathcal{D}\left(\Delta_{2}\right)=\mathcal{D}(\widehat{\Delta})
$$


is the domain of the triangle class $\widehat{\Delta} \in \pi_{2}\left(\Theta_{0}, \Theta_{1}, \Theta_{2}\right)$ so that $n_{p}(\mathcal{D})=-1$ and $n_{j}(\mathcal{D})=0$ for $j=0, \ldots, \kappa$. The above computation then implies that

$$
\begin{aligned}
\underline{\mathfrak{s}}^{0}\left(\mathbf{x}_{0}\right) & =\underline{\mathfrak{s}}^{1}\left(\lambda_{\mathbf{z}}\left(\Delta_{2}\right) \cdot \mathbf{x}_{1}\right)+h_{2} \\
& =\underline{\mathfrak{s}}^{2}\left(\lambda_{\mathbf{z}}\left(\Delta_{0}\right) \lambda_{\mathbf{z}}\left(\Delta_{2}\right) \cdot \mathbf{x}_{2}\right)+h_{0}+h_{2} \\
& =\underline{\mathfrak{s}}^{0}\left(\lambda_{\mathbf{z}}\left(\Delta_{0}\right) \lambda_{\mathbf{z}}\left(\Delta_{1}\right) \lambda_{\mathbf{z}}\left(\Delta_{2}\right) \cdot \mathbf{x}_{0}\right)+h_{0}+h_{1}+h_{2} . \\
& =\underline{\mathfrak{s}}^{0}\left(\lambda_{\mathbf{z}}(\widehat{\Delta}) \cdot \mathbf{x}_{0}\right)+h_{0}+h_{1}+h_{2} . \\
\Rightarrow \quad 0 & =h_{0}+h_{1}+h_{2}-\chi\left(\lambda_{p}\right)=\sum_{i=0}^{3}\left(h_{i}+\chi_{i}\right)
\end{aligned}
$$

This completes the proof of the lemma.

In fact, we may choose the identification of equation 15 for $\operatorname{Spin}^{c}$ classes so that with the notation of the above lemma we have

$$
f_{i}^{\mathfrak{s}}(\mathbf{x} \otimes \mathbf{q}) \in \mathbb{E}_{i-1}\left(\underline{\mathfrak{s}}+\left(m_{i}-1\right) \chi_{i}\right), \quad \forall i \in \frac{\mathbb{Z}}{3 \mathbb{Z}},
$$

or equivalently, $h_{i}=\left(1-m_{i}\right) \chi_{i}$. This last condition determines the triangle classes in a unique way. The closed top generator

$$
\Theta_{i-1} \in \mathrm{CF}\left(L_{i-1}, \nu_{i-1} ; \mathfrak{s}_{i-1}\right) \otimes \mathbb{A}
$$

may then be used to define the map $\mathfrak{f}_{i-1}^{\mathfrak{s}}$ by

$$
\mathfrak{f}_{i-1}^{\mathfrak{s}}: \mathbb{E}_{i}(\mathfrak{s}) \longrightarrow \mathbb{E}_{i+1}(\mathfrak{s}), \quad \mathfrak{f}_{i-1}^{\mathfrak{s}}(\mathbf{x}):=f_{i-1}^{\mathfrak{s}}\left(\mathbf{x} \otimes \Theta_{i-1}\right) .
$$

For a relative $\operatorname{Spin}^{c}$ class $\mathfrak{s} \in \mathfrak{s} \subset \mathfrak{S}$, the restriction of $\mathfrak{f}_{i}^{\mathfrak{s}}$ to $\mathbb{E}_{i+1}(\mathfrak{\mathfrak { s }}) \subset \mathbb{E}_{i+1}(\mathfrak{s})$ will be denoted by $\mathfrak{f}_{i}^{\mathfrak{s}}$. Lemma 8.2 implies that the image of $\mathfrak{f}_{i}^{\mathfrak{s}}$ is in $\mathbb{E}_{i+2}\left(\underline{\mathfrak{s}}+\left(m_{i}-1\right) \chi_{i}\right)$.

Straight forward arguments in Heegaard Floer homology (c.f. section 7 of [OS5]) may be used to show the following proposition, using the closed-ness of the generators $\Theta_{0}, \Theta_{1}$ and $\Theta_{2}$ :

Proposition 8.3. The maps $f_{i}^{\mathfrak{s}}$, for $\mathfrak{s} \in \mathfrak{s} \subset \mathfrak{S}$, as defined above are all chain maps, which are induced by $\mathbb{A}$ chain maps

$$
\mathfrak{f}_{i}^{\mathfrak{s}}: \mathbb{E}_{i+1}(\mathfrak{s}) \longrightarrow \mathbb{E}_{i+2}(\mathfrak{s}), \quad i \in \frac{\mathbb{Z}}{3 \mathbb{Z}}=\{0,1,2\} .
$$

8.2. Compositions in the triangle are null-homotopic. The maps defined in the previous subsection give a triangle of $\mathbb{A}$ chain maps between filtered $(\mathbb{A}, \mathbb{H})$ chain complexes:

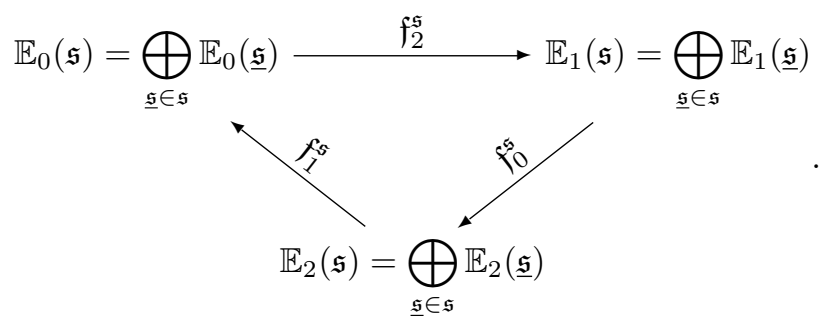

The maps in this triangle change the associated relative $\operatorname{Spin}^{c}$ class in a controlled way, as described in lemma 8.2 . 
Our first observation is the following theorem.

Theorem 8.4. With the notation of the previous sub-section, the compositions $\mathfrak{f}_{i+1}^{\mathfrak{s}} \circ \mathfrak{f}_{i}^{\mathfrak{s}}, i \in \frac{\mathbb{Z}}{3 \mathbb{Z}}=\{0,1,2\}$ from the triangle in equation 18 are $\mathbb{A}$ chain homotopic to zero for each $\operatorname{Spin}^{c}$ class $\mathfrak{s} \in \operatorname{Spin}^{c}(\bar{X})$. More precisely, there are $\mathbb{A}$ homotopy maps

$$
\begin{aligned}
& H_{i}^{\mathfrak{s}}: \mathbb{E}_{i-1}(\mathfrak{s}) \rightarrow \mathbb{E}_{i+1}(\mathfrak{s}), \quad i \in \frac{\mathbb{Z}}{3 \mathbb{Z}}=\{0,1,2\}, \quad \text { s.t. } \\
& H_{i}^{\mathfrak{s}} \circ \partial_{i-1}+\partial_{i+1} \circ H_{i}^{\mathfrak{s}}=\mathfrak{f}_{i-1}^{\mathfrak{s}} \circ \mathfrak{f}_{i+1}^{\mathfrak{s}} \quad \forall i \in \frac{\mathbb{Z}}{3 \mathbb{Z}} .
\end{aligned}
$$

Proof. Throughout this proof, we will assume, for the sake of simplicity, that the top generators $\Theta_{i}, i \in \frac{\mathbb{Z}}{3 \mathbb{Z}}$ are represented by a single intersection point of the corresponding tori. Define the homotopy map $H_{i}^{\mathfrak{s}}$ from the Heegaard quadruple $\left(\Sigma, \boldsymbol{\alpha}, \boldsymbol{\beta}^{i-1}, \boldsymbol{\beta}^{i}, \boldsymbol{\beta}^{i+1}, \mathbf{z}\right)$ by

$$
\begin{aligned}
H_{i}^{\mathfrak{s}} & : \mathbb{E}_{i-1}(\mathfrak{s}) \longrightarrow \mathbb{E}_{i+1}(\mathfrak{s}) \\
H_{i}^{\mathfrak{s}}(\mathbf{x}) & :=\sum_{\substack{\mathbf{y} \in \mathbb{T}_{\alpha} \cap \mathbb{T}_{\beta^{i+1}} \\
\square \in \pi_{2}^{-1}\left(\mathbf{x}, \Theta_{i+1}, \Theta_{i-1}, \mathbf{y}\right)}}\left(\mathfrak{m}(\square) \lambda_{\mathbf{z}}(\square)\right) \cdot \mathbf{y} .
\end{aligned}
$$

Here $\pi_{2}^{j}\left(\mathbf{x}, \Theta_{i+1}, \Theta_{i-1}, \mathbf{y}\right)$ denotes the subset of $\pi_{2}\left(\mathbf{x}, \Theta_{i+1}, \Theta_{i-1}, \mathbf{y}\right)$ consisting of the squares $\square$ with $\mu(\square)=j$, and $\mathfrak{m}(\square)$ denotes the number of points in the moduli space $\mathcal{M}(\square)$, counted with sign. Furthermore $\lambda_{\mathbf{z}}(\square)$ is defined by

$$
\lambda_{\mathbf{z}}(\square)=\lambda_{p}^{n_{p}(\square)} \prod_{j=0}^{\kappa} \lambda_{j}^{n_{j}(\square)} \in \mathbb{A} .
$$

In equation 19, we only count square classes which may be represented as the juxtaposition of the small triangle class $\widehat{\Delta}$ in $\pi_{2}\left(\Theta_{i+1}, \Theta_{i-1}, \Theta_{i}\right)$ with the triangle class $\Delta_{i}$ in $\pi_{2}\left(\mathbf{x}, \Theta_{i}, \mathbf{y}\right)$. We will drop this condition from the notation for the sake of simplicity.

Lemma 8.5. With the above notation fixed, for any relative $\operatorname{Spin}^{c}$ class $\underline{\mathfrak{s}} \in \mathfrak{s} \subset \mathfrak{S}$ the image of

$$
H_{i}^{\mathfrak{s}}=\left.H_{i}^{\mathfrak{s}}\right|_{\mathbb{E}_{i-1}(\mathfrak{s})}: \mathbb{E}_{i-1}(\underline{\mathfrak{s}}) \longrightarrow \mathbb{E}_{i+1}(\mathfrak{s})
$$

is in the sub-complex

$$
\mathbb{E}_{i+1}\left(\underline{\mathfrak{s}}-\left(m_{i} \chi_{i}+\chi_{i-1}+\chi_{i+1}\right)\right) \subset \mathbb{E}_{i+1}(\mathfrak{s}) .
$$

Proof. Without loosing on generality, we may assume that $i=0$. Let $\square \in$ $\pi_{2}^{-1}\left(\mathbf{x}, \Theta_{1}, \Theta_{2}, \mathbf{y}\right)$ be a square connecting $\mathbf{x}$ to $\mathbf{y}$. We can thus find an element $h \in \mathbb{H}$ such that for all such generators and square classes we have

$$
\begin{aligned}
\underline{\mathfrak{s}}^{2}(\mathbf{x}) & =\underline{\mathfrak{s}}^{1}(\mathbf{y})+h+\sum_{i=0}^{2}\left(n_{i}(\square)-n_{p}(\square)\right) \chi_{i}+\sum_{j=3}^{\kappa} n_{j}(\square) \chi_{j} \\
& =\underline{\mathfrak{s}}^{1}(\mathbf{y})+h+\chi\left(\lambda_{p}^{n_{p}(\square)} \prod_{j=0}^{\kappa} \lambda_{j}^{n_{j}(\square)}\right)=\underline{\mathfrak{s}}^{1}\left(\lambda_{\mathbf{z}}(\square) \cdot \mathbf{y}\right)+h .
\end{aligned}
$$


Considering the square classes which are obtained as the juxtaposition of triangles corresponding to $f_{i-1}^{\mathfrak{s}}$ and $f_{i+1}^{\mathfrak{s}}$, and using the coherence of the system of $\operatorname{Spin}^{c}$ classes, we may compute $h$ :

$$
\begin{aligned}
h=h_{2}+h_{1} & =\left(m_{2}-1\right) \chi_{2}+\left(m_{1}-1\right) \chi_{1} \\
& =-\left(m_{0} \chi_{0}+\chi_{1}+\chi_{2}\right) .
\end{aligned}
$$

This completes the proof of the lemma.

If $\mathbf{y}$ is an intersection point in $\mathbb{T}_{\alpha} \cap \mathbb{T}_{\beta^{i+1}}$ and if $\square \in \pi_{2}\left(\mathbf{x}, \Theta_{i+1}, \Theta_{i-1}, \mathbf{y}\right)$ is a square with $\mu(\square)=0$, we may consider the moduli space $\mathcal{M}(\square)$, which is a smooth, oriented 1-dimensional manifold with boundary. The boundary points of this moduli space correspond to different types of degenerations of $\square$. Four types of these degenerations, are degenerations of $\square$ to a bi-gon and a square. Since $\Theta_{i-1}$ and $\Theta_{i+1}$ are closed elements in their corresponding chain complexes, counting such degenerations contribute to the coefficient of $\lambda_{\mathbf{z}}(\square)$.y in the expression

$$
\left(H_{i}^{\mathfrak{s}} \circ \partial_{i-1}+\partial_{i+1} \circ H_{i}^{\mathfrak{s}}\right)(\mathbf{x}) \text {. }
$$

Then we have the possibility of a degeneration of $\square$ as $\Delta \star \Delta^{\prime}$ with $\Delta \in \pi_{2}(\mathbf{x}, \mathbf{q}, \mathbf{y})$ and $\Delta^{\prime} \in \pi_{2}\left(\Theta_{i+1}, \Theta_{i-1}, \mathbf{q}\right)$ for some $\mathbf{q} \in \mathbb{T}_{\beta^{i-1}} \cap \mathbb{T}_{\beta^{i+1}}$ satisfying $\mu(\Delta)=\mu\left(\Delta^{\prime}\right)=0$. Such degenerations correspond to the appearance of $\mathbf{y}$ in the expression

$$
\Psi_{i}\left(\mathbf{x} \otimes \Phi_{i}\left(\Theta_{i+1} \otimes \Theta_{i-1}\right)\right),
$$

where the holomorphic triangle maps $\Psi_{i}$ and $\Phi_{i}$ are defined by

$$
\begin{aligned}
& \Psi_{i}: \mathbb{E}_{i-1}(\mathfrak{s}) \otimes \operatorname{CF}\left(\Sigma, \boldsymbol{\beta}^{i-1}, \boldsymbol{\beta}^{i+1}, \mathbf{z} ; \mathfrak{s}_{i} ; \mathbb{A}\right) \longrightarrow \mathbb{E}_{i+1}(\mathfrak{s}) \\
& \Psi_{i}(\mathbf{x} \otimes \mathbf{p}):=\sum_{\substack{\mathbf{w} \in \mathbb{T}_{\alpha} \cap \mathbb{T}_{\beta^{i+1}} \\
\Delta \in \pi_{2}^{0}(\mathbf{x}, \mathbf{p}, \mathbf{w})}}\left(\mathfrak{m}(\Delta) \lambda_{\mathbf{z}}(\Delta)\right) \mathbf{w} \\
& \Phi_{i}\left(\Theta_{i+1} \otimes \Theta_{i-1}\right):=\sum_{\substack{\mathbf{p} \in \mathbb{T}_{\beta^{i-1}} \cap \mathbb{T}_{\beta^{i+1}} \\
\Delta \in \pi_{2}^{0}\left(\Theta_{i+1}, \Theta_{i-1}, \mathbf{p}\right)}}\left(\mathfrak{m}(\Delta) \lambda_{\mathbf{z}}(\Delta)\right) \mathbf{p} .
\end{aligned}
$$

Since the Heegaard diagram $\left(\Sigma, \boldsymbol{\beta}^{i-1}, \boldsymbol{\beta}^{i}, \boldsymbol{\beta}^{i+1}, \mathbf{z}\right)$ is a standard diagram, one may easily observe that $\Phi_{i}\left(\Theta_{i+1} \otimes \Theta_{i-1}\right)=0$. The reason for this vanishing is that holomorphic triangles which contribute to the above sum come in pairs. This is in fact the same phenomena as what happens in the surgery exact sequence of Ozsváth and Szabó OS3. The relation

$$
\lambda_{p}=\lambda_{0}^{m_{0}-1} \lambda_{1}^{m_{1}-1} \lambda_{2}^{m_{2}-1}
$$

then guarantees that the element of $\mathbb{A}$ associated with both triangles in any pair is the same (note that the corresponding signs associated by the orientation convention are different). Thus, the triangles in each pair will cancel each other to give

$$
\Phi_{i}\left(\Theta_{i+1} \otimes \Theta_{i-1}\right)=0 \Rightarrow \Psi_{i}\left(\mathbf{x} \otimes \Phi_{i}\left(\Theta_{i+1} \otimes \Theta_{i-1}\right)\right)=0 .
$$

Finally, the last type of degeneration for the domain $\square$ is a degeneration of $\square$ as $\square=\Delta \star \Delta^{\prime}$, where $\Delta \in \pi_{2}^{0}\left(\mathbf{x}, \Theta_{i+1}, \mathbf{w}\right)$ and $\Delta^{\prime} \in \pi_{2}^{0}\left(\mathbf{w}, \Theta_{i-1}, \mathbf{y}\right)$ for some $\mathbf{w} \in \mathbb{T}_{\alpha} \cap \mathbb{T}_{\beta^{i}}$. Counting the end points of $\mathcal{M}(\square)$ corresponding to such degenerations gives the coefficient of $\lambda_{\mathbf{z}}(\square) \cdot \mathbf{y}$ in $\left(\mathfrak{f}_{i-1}^{\mathfrak{s}} \circ \mathfrak{f}_{i+1}^{\mathfrak{s}}\right)(\mathbf{x})$. 
Gathering all this data we conclude that the following relation is satisfied.

$$
H_{i}^{\mathfrak{s}} \circ \partial_{i+1}+\partial_{i-1} \circ H_{i}^{\mathfrak{s}}=\mathfrak{f}_{i-1}^{\mathfrak{s}} \circ \mathfrak{f}_{i+1}^{\mathfrak{s}}, \quad \forall i \in \frac{\mathbb{Z}}{3 \mathbb{Z}}=\{0,1,2\} .
$$

implying that $\mathfrak{f}_{i-1}^{\mathfrak{s}} \circ \mathfrak{f}_{i+1}^{\mathfrak{s}}$ is $\mathbb{A}$-chain homotopic to zero, and that the decomposition into relative $\operatorname{Spin}^{c}$ classes in $\mathfrak{S}$ is almost respected by the maps in the sense described in lemmas 8.5 and 8.2 .

8.3. Exactness and computation of chain homotopy type. We would like to apply lemma 3.8 to the triangle of equation 18 . For this purpose, we have to refine the coefficient ring as follows. Let

$$
\mathbb{B}:=\frac{\mathbb{A}\left[\xi_{p}\right]}{\left\langle 1-\lambda_{p} \xi_{p}\right\rangle}
$$

be the algebra constructed from $\mathbb{A}$ by adding an inverse for $\lambda_{p}$. There is a natural homomorphism

$$
\rho: \mathbb{A} \longrightarrow \mathbb{B}
$$

which may be used to take the tensor product of any $\mathbb{A}$ module with $\mathbb{B}$ and construct a $\mathbb{B}$ module from it. We will sometimes denote $\xi_{p}$ by $\lambda_{p}^{-1}$. In the algebra $\mathbb{B}$, the element $\lambda_{i}^{m_{i}-1}$ is invertible, and it thus makes sense to talk about $\lambda_{i}^{1-m_{i}}$. In fact, if $m_{i}>1$ the element $\lambda_{i}$ itself will be invertible. The most interesting case is, however, when some or all of $m_{i}$ are equal to 1 . In particular, if $m_{0}=m_{1}=m_{2}=1$, $\lambda_{p}=1 \in \mathbb{A}$ and consequently we will have $\mathbb{B}=\mathbb{A}$.

We may define, for $i \in \frac{\mathbb{Z}}{3 \mathbb{Z}}=\{0,1,2\}$,

$$
\begin{aligned}
& \mathfrak{g}_{i}^{\mathfrak{s}}: \mathbb{D}_{i+1}(\mathfrak{s}):=\mathbb{E}_{i+1}(\mathfrak{s}) \otimes_{\mathbb{A}} \mathbb{B} \longrightarrow \mathbb{D}_{i-1}(\mathfrak{s}):=\mathbb{E}_{i-1}(\mathfrak{s}) \otimes_{\mathbb{A}} \mathbb{B} \\
& \mathfrak{g}_{i}^{\mathfrak{s}}(a)=\lambda_{i}^{1-m_{i}} \cdot f_{i}^{\mathfrak{s}}(a), \quad \forall a \in \mathbb{D}_{i+1}(\mathfrak{s}) .
\end{aligned}
$$

From lemma 8.2 we know that $\mathfrak{g}_{i}^{\mathfrak{s}}$ is a filtered $(\mathbb{B}, \mathbb{H})$ map between filtered $(\mathbb{B}, \mathbb{H})$ chain complexes $\mathbb{D}_{i+1}(\mathfrak{s})$ and $\mathbb{D}_{i-1}(\mathfrak{s})$ which decomposes as a sum of maps

$$
\mathfrak{g}_{i}^{\mathfrak{s}}: \mathbb{D}_{i+1}(\underline{\mathfrak{s}}) \longrightarrow \mathbb{D}_{i-1}(\underline{\mathfrak{s}}), \quad \forall \underline{\mathfrak{s}} \in \mathfrak{s} \subset \mathfrak{S} .
$$

We may also modify the maps $H_{i}^{\mathfrak{s}}$ so that they respect the relative $\mathrm{Spin}^{c}$ decompositions. According to lemma 8.5 the following definition gives the appropriate chain homotopy maps

$$
\begin{aligned}
& G_{i}^{\mathfrak{s}}=\bigoplus_{\underline{\mathfrak{s}} \in \mathfrak{s}} G_{i}^{\mathfrak{s}}: \mathbb{D}_{i-1}(\mathfrak{s})=\bigoplus_{\underline{\mathfrak{s}} \in \mathfrak{s}} \mathbb{D}_{i-1}(\underline{\mathfrak{s}}) \longrightarrow \mathbb{D}_{i+1}(\mathfrak{s})=\bigoplus_{\underline{\mathfrak{s}} \in \mathfrak{s}} \mathbb{D}_{i+1}(\underline{\mathfrak{s}}) \\
& G_{i}^{\mathfrak{s}}(a)=\left(\xi_{p} \lambda_{i}^{m_{i}-1}\right) \cdot H_{i}^{\mathfrak{s}}(a), \quad \forall a \in \mathbb{D}_{i-1}(\mathfrak{s}) .
\end{aligned}
$$

Theorem 8.6. With our previous notation fixed and for any $i \in \frac{\mathbb{Z}}{3 \mathbb{Z}}=\{0,1,2\}$, the map from $\mathbb{D}_{i}(\mathfrak{s})$ to the mapping cone of $\mathfrak{g}_{i}^{\mathfrak{s}}$ defined by

$$
\begin{aligned}
& \mathcal{I}_{i}^{\mathfrak{s}}: \mathbb{D}_{i}(\mathfrak{s}) \longrightarrow \mathbb{D}_{i+1}(\mathfrak{s}) \oplus \mathbb{D}_{i-1}(\mathfrak{s}) \\
& \mathcal{I}_{i}^{\mathfrak{s}}(\mathbf{z}):=\left(\mathfrak{g}_{i-1}^{\mathfrak{s}}(\mathbf{z}), G_{i+1}^{\mathfrak{s}}(\mathbf{z})\right)
\end{aligned}
$$

is a filtered chain homotopy equivalence of filtered $(\mathbb{B}, \mathbb{H})$ chain complexes. In particular, the decompositions of the two sides into relative $\operatorname{Spin}^{c}$ classes $\mathfrak{\mathfrak { s }} \in \mathfrak{s} \subset \mathfrak{S}$ is respected by this chain homotopy equivalence. 
Proof. For any integer $j \in \mathbb{Z}$ let us define

$$
A_{j}:= \begin{cases}\mathbb{D}_{0}(\mathfrak{s}) & \text { if } j=0(\bmod 3) \\ \mathbb{D}_{1}(\mathfrak{s}) & \text { if } j=1(\bmod 3) \\ \mathbb{D}_{2}(\mathfrak{s}) & \text { if } j=2(\bmod 3)\end{cases}
$$

Denote the differential of $A_{j}$ by $d_{j}$. Furthermore, define $f_{j}: A_{j} \rightarrow A_{j+1}$ to be $\mathfrak{g}_{2}^{\mathfrak{s}}, \mathfrak{g}_{0}^{\mathfrak{s}}$ or $\mathfrak{g}_{1}^{\mathfrak{s}}$ for $j=0,1$ or 2 modulo 3 , respectively. Let $H_{j}: A_{j} \rightarrow A_{j+2}$, depending on whether $j=0,1$ or 2 modulo 3 be the maps $G_{1}^{\mathfrak{s}}, G_{2}^{\mathfrak{s}}$ and $G_{0}^{\mathfrak{s}}$, respectively. By lemma 3.8 , in order to show that the map $\mathcal{I}_{i}^{\mathfrak{s}}$ is a chain homotopy equivalence of filtered $(\mathbb{B}, \mathbb{H})$ chain complexes we have to show that the differences $\phi_{i}=f_{i+2} \circ H_{i}-H_{i+1} \circ f_{i}: A_{i} \rightarrow A_{i+3}$ are chain homotopy equivalences. Checking that all the constructions respect the decomposition into relative $\operatorname{Spin}^{c}$ classes in $\mathfrak{S}$ is straight-forward from the lemmas 8.2 and 8.5 .

As in [OS6] and OS3], checking the above claim is done by considering holomorphic pentagons associated with Heegaard diagrams of the form

$$
\left(\Sigma, \boldsymbol{\alpha}, \boldsymbol{\beta}^{j}, \boldsymbol{\beta}^{j+1}, \boldsymbol{\beta}^{j+2}, \boldsymbol{\beta}^{j+3}, \mathbf{z}\right),
$$

where $\boldsymbol{\beta}^{j}$ denotes a set of $\ell$ simple closed curves which are Hamiltonian isotopes of the curves in $\boldsymbol{\beta}^{i}$ where $i \in \frac{\mathbb{Z}}{3 \mathbb{Z}}$ is equal to 0,1 or 2 and $j$ is congruent to $i$ modulo 3. Let us denote the top generator of the Heegaard Floer homology group associated with $\left(\Sigma, \boldsymbol{\beta}^{j}, \boldsymbol{\beta}^{j+1}, \mathbf{z}\right)$ by $\Theta_{j}$, by little abuse of notation. More generally, the top generator associated with $\left(\Sigma, \boldsymbol{\beta}^{i}, \boldsymbol{\beta}^{j}, \mathbf{z}\right)$ will be denoted by $\Theta_{i j}$. For any three indices $i<j<k$, there is a triangle, with small area (assuming that the Hamiltonian isotopies changing the curve collection to each other are small) which connects $\Theta_{i j}, \Theta_{j k}$ and $\Theta_{i k}$. Denote this triangle class by $\Delta_{i j k}$.

Without loosing on generality, we may assume that $j=0$ modulo 3 . Choose a generator $\mathbf{x} \in \mathbb{T}_{\alpha} \cap \mathbb{T}_{\beta^{j}}$ so that $\underline{\mathfrak{s}}(\mathbf{x})=\underline{\mathfrak{s}} \in \mathfrak{s}$. The curves in $\boldsymbol{\beta}^{j+3}$ are Hamiltonian isotopes of those in $\boldsymbol{\beta}^{j}$. Thus there is a natural closest point map

$$
I: \mathbb{T}_{\alpha} \cap \mathbb{T}_{\beta^{j}} \rightarrow \mathbb{T}_{\alpha} \cap \mathbb{T}_{\beta^{j+3}}
$$

There is a natural triangle class connecting $\Theta_{j, j+3}, \mathbf{x}$ and $I(\mathbf{x})$ which will be denoted by $\Delta_{\mathrm{x}}$.

Let us denote the complex associated with $\left(\Sigma, \boldsymbol{\beta}_{j}, \boldsymbol{\beta}_{j+1}, \mathbf{z}\right)$ and the coefficient ring $\mathbb{B}$ with $B_{j}$, and the complex associated with $\left(\Sigma, \boldsymbol{\beta}_{j}, \boldsymbol{\beta}_{j+2}, \mathbf{z}\right)$ (again with coefficient ring $\mathbb{B})$ by $C_{j}$, and finally the complex associated with $\left(\Sigma, \boldsymbol{\beta}_{j}, \boldsymbol{\beta}_{j+3}, \mathbf{z}\right)$ by $D_{j}$. We omit the straight forward details of the definitions.

Define a map $\mathcal{P}_{j}: A_{j} \rightarrow A_{j+3} \cong A_{j}$ by

$$
\mathcal{P}_{j}(\mathbf{x})=\sum_{\substack{\mathbf{y} \in \mathbb{T}_{\alpha} \cap \mathbb{T}_{\beta_{j+3}} \\ \square \in \pi_{2}^{-2}\left(\mathbf{x}, \Theta_{j+1}, \Theta_{j+2}, \Theta_{j+3}, \mathbf{y}\right)}}\left(\mathfrak{m}(\bullet) \xi_{p} \lambda_{\mathbf{z}}(\bullet)\right) \mathbf{y} .
$$

The class of the pentagons counted in the above sum is determined by juxtaposing a triangle class $\Delta_{\mathbf{x}} \in \pi_{2}\left(\mathbf{x}, \Theta_{j, j+3}, I(\mathbf{x})\right)$ with an standard square class $\widehat{\square} \in \pi_{2}\left(\Theta_{j}, \Theta_{j+1}, \Theta_{j+2}, \Theta_{j, j+3}\right)$ with small area. As usual, we will drop this class 
from the notation.

Let us assume that $\square \in \pi_{2}^{-1}\left(\mathbf{x}, \Theta_{j+1}, \Theta_{j+2}, \Theta_{j+3}, \mathbf{y}\right)$ is a pentagon class which has Maslov index -1. Consider the ends of the smooth orientable one dimensional moduli space $\mathcal{M}(\bullet)$, which correspond to the degenerations discussed in theorem 6.8.

Considering the possible degenerations at the boundary of $\mathcal{M}(\bullet)$, theorem 6.8 implies

$$
\begin{aligned}
\phi_{j}\left(a_{j}\right)= & \left(\mathcal{P}_{j} \circ d_{j}-d_{j} \circ \mathcal{P}_{j}\right)\left(a_{j}\right) \\
& +\xi_{p} . I_{j}\left(a_{j} \otimes K_{j}\left(\Theta_{j} \otimes \Theta_{j+1} \otimes \Theta_{j+2}\right)\right), \quad \forall a_{j} \in A_{j},
\end{aligned}
$$

where the maps $I_{j}: A_{j} \otimes D_{j} \rightarrow A_{j+3}$ and $K_{j}\left(\Theta_{j} \otimes \Theta_{j+1} \otimes \Theta_{j+2}\right)$ are defined as follows.

$$
\begin{aligned}
& I_{j}(\mathbf{x} \otimes \mathbf{q}):=\sum_{\mathbf{y} \in \mathbb{T}_{\alpha} \cap \mathbb{T}_{\beta^{j+3}}} \sum_{\Delta \in \pi_{2}^{0}(\mathbf{x}, \mathbf{q}, \mathbf{y})}\left(\mathfrak{m}(\Delta) \lambda_{\mathbf{w}}(\Delta)\right) \mathbf{y} \\
& K_{j}\left(\Theta_{j} \otimes \Theta_{j+1} \otimes \Theta_{j+2}\right):=\sum \sum_{\substack{\mathbf{q} \in \mathbb{T}_{\beta} \cap \mathbb{T}_{\beta^{j+3}} \\
\square \in \pi_{2}^{-1}\left(\Theta_{j}, \Theta_{j+1}, \Theta_{j+2}, \mathbf{q}\right)}}\left(\mathfrak{m}(\square) \lambda_{\mathbf{z}}(\square)\right) \mathbf{q} .
\end{aligned}
$$

Two of the terms appearing in theorem 6.8 vanish and are not present in the equation 20. These are the terms that correspond to degenerations containing a triangle in $\pi_{2}\left(\Theta_{j}, \Theta_{j+1}, \mathbf{q}\right)$ for some $\mathbf{q} \in \mathbb{T}_{\beta^{j}} \cap \mathbb{T}_{\beta^{j+2}}$, or a triangle in $\pi_{2}\left(\Theta_{j+1}, \Theta_{j+2}, \mathbf{q}\right)$ for some $\mathbf{q} \in \mathbb{T}_{\beta^{j+1}} \cap \mathbb{T}_{\beta^{j+3}}$. The total contribution of such triangles vanishes, since they come in canceling pairs. Thus the terms containing such degenerations would vanish as well.

Note that the map $\mathbf{x} \mapsto I_{j}\left(\mathbf{x} \otimes \Theta_{j, j+3}\right)$ is a perturbation of the isomorphism $I$ with a map $\epsilon: A_{j} \rightarrow A_{j+3}$ which takes a generator $\mathbf{x}$ to generators with smaller energy than $I(\mathbf{x})$, when we equip $A_{j+3}$ with an appropriate energy filtration. This follows since the contributions from triangle classes other than $\Delta_{\mathbf{x}}$ will contribute more than the small energy associated with $\Delta_{\mathbf{x}}$. Standard arguments in Heegaard Floer theory (c.f. Ozsváth and Szabó's original paper OS5) may then be applied to construct an explicit inverse for this map up to filtered $(\mathbb{B}, \mathbb{H})$ chain homotopy. In order to complete the proof of the theorem, it is thus enough to show that

$$
K_{j}\left(\Theta_{j} \otimes \Theta_{j+1} \otimes \Theta_{j+2}\right)=\lambda_{p} \Theta_{j, j+3} .
$$

This can be proved directly, since the Heegaard quadruple

$$
\left(\Sigma, \boldsymbol{\beta}^{j}, \boldsymbol{\beta}^{j+1}, \boldsymbol{\beta}^{j+2}, \boldsymbol{\beta}^{j+3}, \mathbf{z}\right)
$$

is a special Heegaard diagram, which may be analyzed without too much difficulty. The only difference with earlier considerations of Ozsváth and Szabó (e.g. in OS6, subsection 4.2) is the following. There is a preferred square class which contributes to the second sum of equation 21. This square class has small total area, and multiplicity 1 at $p$. The contribution of this class would give $\lambda_{p} \Theta_{j, j+3}$. The rest of contributing square classes come in pairs and the elements of $\mathbb{B}$ associated with both elements in each pair are the same (with opposite sign), since the relation

$$
\lambda_{p}=\lambda_{0}^{m_{0}-1} \lambda_{1}^{m_{1}-1} \lambda_{2}^{m_{2}-1}
$$


is satisfied in $\mathbb{A}$ and hence in $\mathbb{B}$. Thus the two square classes in each pair cancel each other.

8.4. Special cases. Let us now consider a few special cases, which correspond to the existing exact sequences in Heegaard Floer homology. We use the observation of this subsection as an indication of how the new language developed in this paper for understanding Heegaard Floer theory of Ozsváth and Szabó may be used to understand the existing objects in a uniform way, and extend them to the more general setup.

The first case we would like to consider, is the case where $m_{0}=m_{1}=m_{2}=1$. This would be the case if $\mu_{0}$ and $\mu_{1}$ cut each other in a single transverse point (so that $\left.\#\left(\mu_{0} \cdot \mu_{1}\right)=1\right)$ and $\mu_{2}=-\left(\mu_{0}+\mu_{1}\right)$. In this case, we will have

$$
\begin{aligned}
& \mathbb{A}=\frac{\widehat{\mathbb{A}}}{\left\langle\lambda_{p}=\lambda_{0}^{m_{0}-1} \lambda_{1}^{m_{1}-1} \lambda_{2}^{m_{2}-1}\right\rangle}=\frac{\widehat{\mathbb{A}}}{\left\langle\lambda_{p}=1\right\rangle}, \quad \& \\
& \mathbb{B}=\frac{\mathbb{A}\left[\xi_{p}\right]}{\left\langle\xi_{p} \lambda_{p}=1\right\rangle}=\mathbb{A}=\frac{\widehat{\mathbb{A}}}{\left\langle\lambda_{p}=1\right\rangle} .
\end{aligned}
$$

Thus the algebra $\mathbb{B}$ is the algebra associated with any of the diagrams

$$
\left(\Sigma, \boldsymbol{\alpha}, \boldsymbol{\beta}^{i}, \mathbf{z}-\{p\}\right), \quad i=0,1,2 .
$$

In particular, if the simple closed curve $\lambda$ determines the surgery, and $\mu_{0}, \mu_{1}$ and $\mu_{2}$ correspond to $\infty, 0$ and 1 surgeries respectively, the above conditions are satisfied. For an arbitrary ring $R$ such that there is a ring homomorphism $\rho_{R}: \mathbb{B} \rightarrow R$, one may define the $R$ chain complexes

$$
\mathbb{D}_{i}(\mathfrak{s} ; R)=\mathbb{D}_{i}(\mathfrak{s}) \otimes_{\mathbb{B}} R, \quad i=0,1,2 .
$$

The triangle of theorem 8.6 gives a triangle of $R$ chain maps between $\mathbb{D}_{i}(\mathfrak{s} ; R)$, $i=0,1,2$, and the conclusion of the theorem remains true (however, we may need to drop the filtration from the conclusions if $\rho_{R}$ does not respect the filtration). In particular, the homology groups

$$
\mathbb{H}_{i}(\mathfrak{s} ; R):=H_{*}\left(\mathbb{D}_{i}(\mathfrak{s} ; R), \partial_{\mathbb{D}_{i}}\right), \quad i=0,1,2
$$

fit into an exact triangle. The exact triangle of [Ef4] and [Ef1] is a special case of such exact triangles.

Consider the algebra $\overline{\mathbb{A}}$ and the quotient map $r: \mathbb{A}=\mathbb{B} \rightarrow \overline{\mathbb{A}}$ where

$$
\overline{\mathbb{A}}=\frac{\mathbb{A}}{\left\langle\lambda_{0}=\lambda_{1}=1\right\rangle}=\frac{\mathbb{B}}{\left\langle\lambda_{0}=\lambda_{1}=1\right\rangle} .
$$

Correspondingly, let $\overline{\mathbb{H}}$ be the quotient of $\mathbb{H}$ by the action of $\chi_{0}, \chi_{1}$ and $\chi_{2}$. From the filtered $(\mathbb{B}, \mathbb{H})$ chain complexes $\mathbb{D}_{i}(\mathfrak{s})$ we may construct the filtered $(\overline{\mathbb{A}}, \overline{\mathbb{H}})$ chain complexes

$$
\mathbb{C}_{i}(\mathfrak{s}):=\mathbb{D}_{i}(\mathfrak{s}) \otimes_{\mathbb{B}} \overline{\mathbb{A}}, \quad i \in \frac{\mathbb{Z}}{3 \mathbb{Z}}=\{0,1,2\}
$$

The complex $\mathbb{C}_{i}(\mathfrak{s})$ may be identified as $\mathrm{CF}\left(X_{\tau^{i}}(1), \tau^{i}(1), \mathfrak{s}\right)$ when $i=0,1$ and as $\mathrm{CF}\left(X_{\tau^{2}}(2), \tau^{2}(2), \mathfrak{s}\right)$ when $i=2$. Here $X_{\tau^{i}}(j)$ denotes the three-manifold obtained from $X$ by filling out the $j$-th suture in $\tau^{i}$ and $\tau^{i}(j)$ denotes the induced set of sutures on the boundary of $X_{\tau^{i}}(j)$. The triangle of theorem 8.6 thus generalizes the 
exact sequence in homology, which appears as theorem $1.7 \mathrm{in}$ OS5. Also, theorem 4.7 from OS6 is a special case of theorem 8.6. Furthermore, theorem 8.2 from OS1 is also a corollary in this situation.

Let us now assume that $m_{1}=m_{2}=1$, while $m_{0}=m$ is an arbitrary integer. In particular, if $\mu_{0}, \mu_{1}$ and $\mu_{2}$ correspond to the surgery coefficients $\infty, n$ and $n+m$, for some integer $n \in \mathbb{Z}$ this would be the case. We will thus have

$$
\mathbb{A}=\frac{\widehat{\mathbb{A}}}{\left\langle\lambda_{p}=\lambda_{0}^{m-1}\right\rangle} \text {. }
$$

We may thus define the following quotient ring of $\mathbb{B}$ :

$$
\mathbb{A}_{m}=\frac{\widehat{\mathbb{A}}}{\left\langle\lambda_{p}-\lambda_{0}^{m-1}, \lambda_{0}^{m}-1\right\rangle}=\frac{\mathbb{B}}{\left\langle\xi_{p}=\lambda_{0}\right\rangle} .
$$

The filtration module $\mathbb{H}=\mathrm{H}^{2}(X, \partial X ; \mathbb{Z})$ and the space $\mathfrak{S}=\operatorname{Spin}^{c}(X, \tau)$ corresponding to the coefficient ring $\mathbb{B}$ should be changed to

$$
\begin{aligned}
\mathbb{H}_{m} & :=\frac{\mathbb{H}}{\left\langle m \chi_{0}\right\rangle_{\mathbb{Z}}}=\frac{\mathrm{H}^{2}(X, \partial X ; \mathbb{Z})}{\left\langle m \chi_{0}\right\rangle_{\mathbb{Z}}}, \quad \& \\
\mathfrak{S}_{m} & :=\frac{\mathfrak{S}}{\left\langle m \chi_{0}\right\rangle_{\mathbb{Z}}}=\frac{\operatorname{Spin}^{c}(X, \tau)}{\left\langle m \chi_{0}\right\rangle_{\mathbb{Z}}} .
\end{aligned}
$$

Theorem 8.6 then gives the main result of [Ef3] as a special case. The surgery exact sequence of theorem 3.1 in OS3] is in turn a consequence of this last result. In a similar way, theorem 6.2 from OS4 follows from this last consideration. 


\section{REFERENCES}

[Ef1] Eftekhary, E., A combinatorial approach to surgery formulas in Heegaard Floer homology, Algebr. Geom. Topol. 9 (2009), no. 4, 2225-2246.

[Ef2] Eftekhary, E., Longitude Floer homology and the Whitehead double, Algebr. Geom. Topol. 5 (2005), 1389-1418.

[Ef3] Eftekhary, E., Knot Floer homology and Morse surgery, preprint, available at arXiv:math/0603171.

[Ef4] Eftekhary, E., Floer homology and splicing knot complements preprint, available at arXiv:0802.2874.

[Gab1] Gabai, D., Foliations an the topology of 3-manifolds, J. Differential Geom. 18 (1983) 445-503.

[Gab2] Gabai, D., Foliations an the topology of 3-manifolds. II, J. Differential Geom. 26 (1987) 461-478.

[Gab3] Gabai, D., Foliations an the topology of 3-manifolds. III, J. Differential Geom. 26 (1987) 479-536.

[Gab2] Gabai, D., Detecting fibered links in $S^{3}$, Comment. Math. Helv. 61 (1986) 519-555.

[HKM1] Honda, K., Kazez, W. H., Matić, G., Convex decomposition theory. Int. Math. Res. Not. 2002, no. 2, 55-88.

[HKM2] Honda, K., Kazez, W. H., Matić, G., The contact invariant in sutured Floer homology, Invent. Math. 176 (2009), no. 3, 637-676.

[Ju1] Juhasz, A., Holomorphic discs and sutured manifolds, Algebr. Geom. Topol. 6 (2006), 1429-1457.

[Ju2] Juhasz, A., Floer homology and surface decompositions, Geom. Topol. 12 (2008), no. $1,299-350$.

[Ju3] Juhasz, A., The sutured Floer homology polytope, Geom. Topol. 14 (2010), no. 3, 1303-1354.

[Lip] Lipshitz, R., A cylindrical reformulation of Heegaard Floer homology, Geom. Topol. 10 (2006), 955-1097.

[LOT1] Lipshitz, R., Ozsváth, P., Thurston, D., Bordered Heegaard Floer homology: Invariance and pairing, preprint, available at arXiv:0810.0687.

[LOT2] Lipshitz, R., Ozsváth, P., Thurston, D., Bimodules in bordered Heegaard Floer homology, preprint, available at arXiv:1003.0598.

[LOSS] Lisca, P., Ozsváth, P., Stipsicz, A. I., Szabó, Z., Heegaard Floer invariants of Legendrian knots in contact three-manifolds, J. Eur. Math. Soc. (JEMS) 11 (2009), no. 6, 13071363.

[Ni1] Ni, Y., Link Floer homology detects the Thurston norm, Geom. Topol. 13 (2009), no. 5, 2991-3019.

[Ni2] Ni, Y., Heegaard Floer homology and fibred 3-manifolds, Amer. J. Math. 131 (2009), no. 4, 1047-1063.

[Ni3] Ni, Y., Knot Floer homology detects fibred knots, Invent. Math. 170 (2007), no. 3, 577-608.

[OS1] Ozsváth, P., Szabó, Z., Holomorphic disks and knot invariants, Adv. Math. 186 (2004), no. 1, 58-116.

[OS2] Ozsváth, P., Szabó, Z., Heegaard Floer homology and alternating knots, Geometry $\&$ Topology, Vol. 7 (2003), 225-254.

[OS3] Ozsváth, P., Szabó, Z., Knot Floer homology and integer surgeries, Algebr. Geom. Topol. 8 (2008), no. 1, 101-153.

[OS4] Ozsváth, P., Szabó, Z., Knot Floer homology and rational surgeries, Algebr. Geom. Topol. 11 (2011), no. 1, 1-68.

[OS5] Ozsváth, P., Szabó, Z., Holomorphic disks and topological invariants for closed threemanifolds, Ann. of Math. (2) 159 (2004), no. 3, 1027-1158.

[OS5] Ozsváth, P., Szabó, Z., Holomorphic disks and three-manifold invariants: properties and applications, Ann. of Math. (2) 159 (2004), no. 3, 1159-1245.

[OS6] Ozsváth, P., Szabó, Z., On the Heegaard Floer homology of branched double-covers, Adv. Math. 194 (2005), no. 1, 1-33.

[OS7] Ozsváth, P., Szabó, Z., Holomorphic disks and genus bounds, Geometry $\&$ Topolology 8 (2004), 311-334. 
[OS8] Ozsváth, P., Szabó, Z., Holomorphic disks, link invariants and the multi-variable Alexander polynomial, Algebr. Geom. Topol. 8 (2008), no. 2, 615-692.

[OS9] Ozsváth, P., Szabó, Z., Link Floer homology and the Thurston norm. J. Amer. Math. Soc. 21 (2008), no. 3, 671-709.

[OS10] Ozsváth, P., Szabó, Z., Heegaard Floer homology and contact structures, Duke Math. J. 129 (2005), no. 1, 39-61.

[OS11] Ozsváth, P., Szabó, Z., Holomorphic triangles and invariants for smooth four-manifolds, Adv. Math. 202 (2006), no. 2, 326-400.

[Ras1] Rasmussen, J., Floer homology of surgeries on two bridge knots, Alg. and Geo. Topology 2 (2002) 757-789

[Ras2] Rasmussen, J., Floer homology and knot complements, Ph.D thesis, Harvard univ., also available at math.GT/0306378

Department of Mathematics, Sharif University of Technology, P. O. Box 11155-9415, TeHran, Iran

E-mail address: akram.alishahi@gmail.com

School of Mathematics, Institute for Research in Fundamental Science (IPM), P. O. Box 19395-5746, TeHran, Iran

E-mail address: eaman@ipm.ir 